Supporting Information for

\title{
A Diverse Library of Chiral Cyclopropane Scaffolds via \\ Chemoenzymatic Assembly and Diversification of
}

\section{Cyclopropyl Ketones}

Donggeon Nam, Viktoria Steck, Robert J. Potenzino and Rudi Fasan*

Department of Chemistry, University of Rochester, Rochester, NY 14627, United States.

Correspondence should be addressed to R.F. (rfasan@ur.rochester.edu)

Table of contents:

Supplementary Table S1-S3

Pages S2-S3

Supplementary Figures S1-S6

Pages S4-S49

Analytical Methods

Pages S50-S52

Compound Characterization Data

Pages S53-S75

X-Ray Crystallographic Data

Pages S76-78

NMR spectra

Pages S79-S134

References

Page S135 
Table S1. Activity of wild-type sperm whale myoglobin $(\mathrm{Mb})$ and other hemoproteins in the cyclopropanation of styrene with 1-diazo-3-phenyl-2-propanone 2 using optimized reaction conditions. $^{\text {a }}$

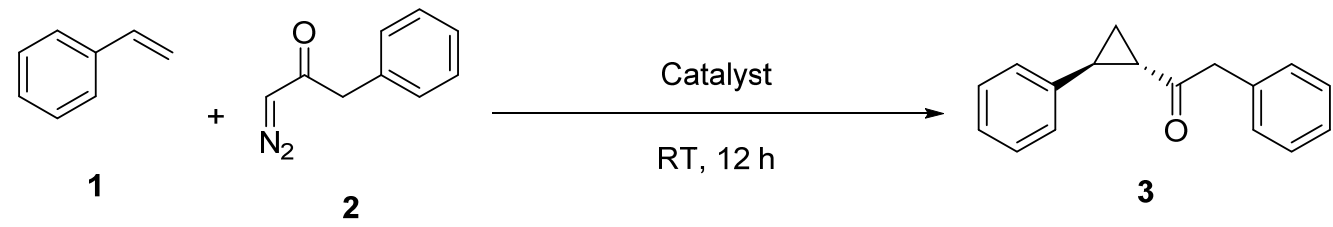

\begin{tabular}{|c|c|c|c|c|}
\hline Catalyst & $\begin{array}{l}\text { Yield }^{b} \\
(\mathrm{SFC})\end{array}$ & TON & $\% \mathrm{de}$ & $\% \mathrm{de}$ \\
\hline none & $0 \%$ & - & - & - \\
\hline $\mathrm{Rh}_{2}(\mathrm{OAc})_{4}$ & $<1 \%{ }^{c}$ & n.d. & n.d. & n.d. \\
\hline $\mathrm{Co}(\mathrm{TPP})$ & $<1 \%{ }^{c}$ & n.d. & n.d. & n.d. \\
\hline $\mathrm{Fe}(\mathrm{TPP}) \mathrm{Cl}$ & $<1 \%{ }^{d}$ & n.d. & n.d. & n.d. \\
\hline Hemin & $<1 \%$ & n.d. & n.d. & n.d. \\
\hline $\mathrm{Mb}(\mathrm{WT})$ & trace & n.d. & n.d. & n.d. \\
\hline Catalase & $1 \%$ & 4 & n.d. & n.d. \\
\hline Cytochrome $c$ (equine heart) & trace & n.d. & n.d. & n.d. \\
\hline Р450вм3 & $2 \%$ & 8 & n.d. & n.d. \\
\hline XplA & trace & n.d. & n.d. & n.d. \\
\hline BezE & trace & n.d. & n.d. & n.d. \\
\hline
\end{tabular}


Table S2. Catalytic activity of additional single-site Mb variants for the cyclopropanation of styrene with 1-diazo-3-phenyl-2-propanone (2) under optimized reaction conditions.

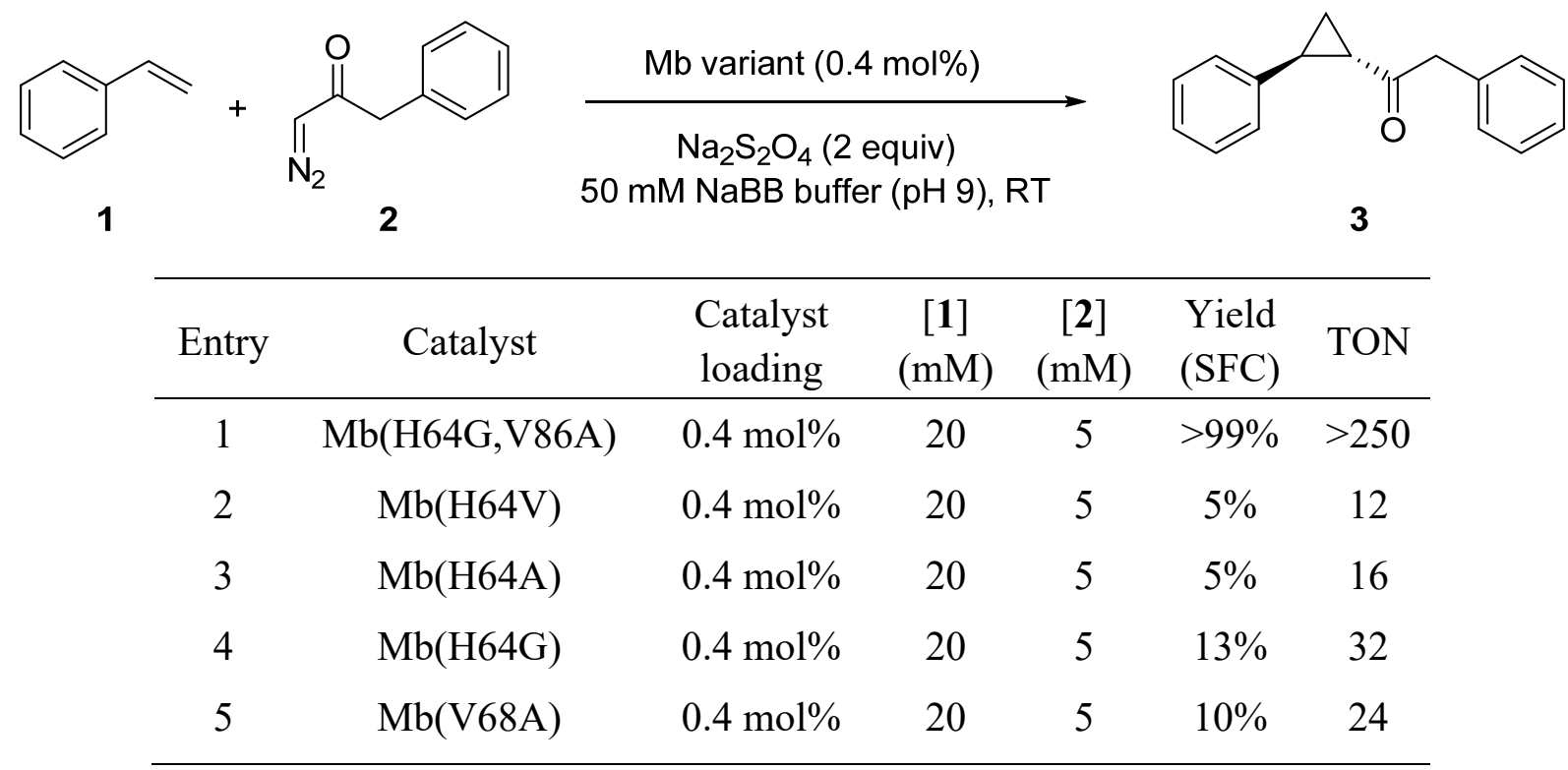

Table S3. $\mathrm{pH}$ dependence of $\mathrm{Mb}(\mathrm{H} 64 \mathrm{G}, \mathrm{V} 68 \mathrm{~A})$-catalyzed cyclopropanation of styrene with 1diazo-3-phenyl-2-propanone (2).

\begin{tabular}{|c|c|c|c|c|c|c|c|}
\hline 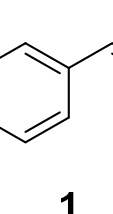 & $+\prod_{N_{2}}$ & $\frac{0.4 \mathrm{~mol}}{\mathrm{Na}}$ & $\begin{array}{l}\mathrm{Mb}(\mathrm{H} 64 \\
{ }_{2} \mathrm{O}_{4}(2 \mathrm{e} \\
\text { Iffer }(\mathrm{pH}\end{array}$ & $\begin{array}{l}\text { G,V68A) } \\
\text { quiv) } \\
\text {-10), RT }\end{array}$ & 1 & 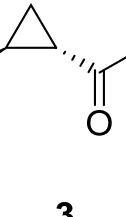 & \\
\hline Entry & Catalyst & $\begin{array}{l}\text { Catalyst } \\
\text { loading }\end{array}$ & $\begin{array}{c}{[\mathbf{1}]} \\
(\mathrm{mM})\end{array}$ & $\begin{array}{c}{[2]} \\
(\mathrm{mM})\end{array}$ & Buffer & $\begin{array}{l}\text { Yield } \\
(\mathrm{SFC})\end{array}$ & TON \\
\hline 1 & Mb(H64G,V68A) & $0.4 \mathrm{~mol} \%$ & 20 & 5 & KPi pH 7 & $64 \%$ & 160 \\
\hline 2 & $\mathrm{Mb}(\mathrm{H} 64 \mathrm{G}, \mathrm{V} 68 \mathrm{~A})$ & $0.4 \mathrm{~mol} \%$ & 20 & 5 & KPi pH 8 & $84 \%$ & 210 \\
\hline 3 & $\mathrm{Mb}(\mathrm{H} 64 \mathrm{G}, \mathrm{V} 68 \mathrm{~A})$ & $0.4 \mathrm{~mol} \%$ & 20 & 5 & $\mathrm{NaBB}$ pH 9 & $>99 \%$ & $>250$ \\
\hline 4 & $\mathrm{Mb}(\mathrm{H} 64 \mathrm{G}, \mathrm{V} 68 \mathrm{~A})$ & $0.4 \mathrm{~mol} \%$ & 20 & 5 & NaBB pH 10 & $60 \%$ & 150 \\
\hline
\end{tabular}


Figure S1. Time-course analysis of Mb(H64G,V68A)-catalyzed cyclopropanation of styrene (1) and styrene derivatives 4a-4d with 1-diazo-3-phenyl-2-propanone (2). Reaction conditions: $10 \mu \mathrm{M}$ enzyme, $10 \mathrm{mM}$ styrene, $2.5 \mathrm{mM}$ diazoketone, $10 \mathrm{mM} \mathrm{Na} 2 \mathrm{~S}_{2} \mathrm{O}_{4}$ in $50 \mathrm{mM}$ sodium borate buffer (pH 9.0) with $10 \% \mathrm{EtOH}$, room temperature, anaerobic atmosphere. Reactions were quenched with $50 \mu \mathrm{L} \mathrm{3M} \mathrm{HCl}$, followed by DCM extraction and chiral SFC analysis.

(a) Time-course analysis of $\mathrm{Mb}(\mathrm{H} 64 \mathrm{G}, \mathrm{V} 68 \mathrm{~A})$-catalyzed cyclopropanation of styrene 1 with 1diazo-3-phenyl-2-propanone to give cyclopropane 3 :

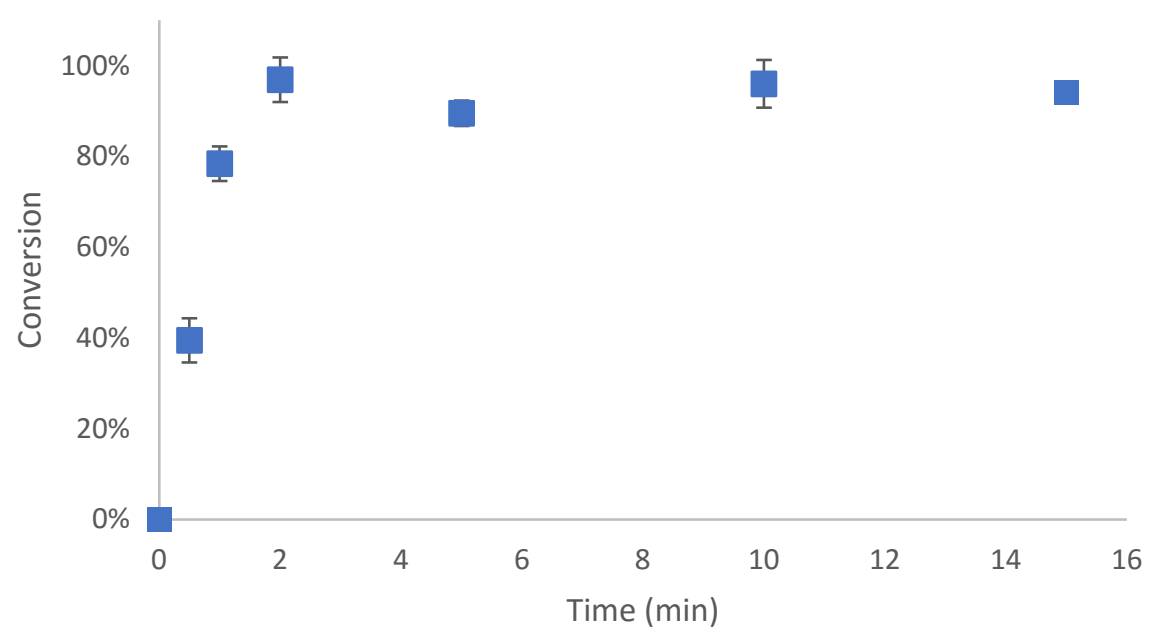

(b) Time-course analysis of $\mathrm{Mb}(\mathrm{H} 64 \mathrm{G}, \mathrm{V} 68 \mathrm{~A})$-catalyzed cyclopropanation of p-methyl-styrene 4a with 1-diazo-3-phenyl-2-propanone to give cyclopropane 5a:

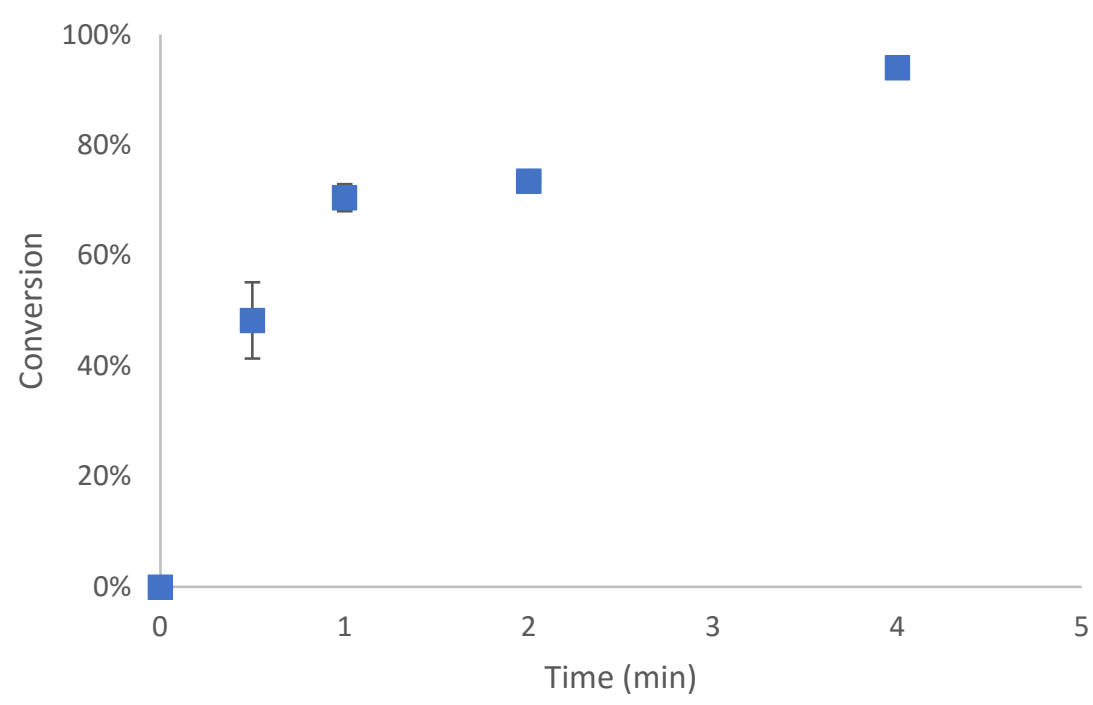


(c) Time-course analysis of Mb(H64G,V68A)-catalyzed cyclopropanation of p-methoxy-styrene 4b with 1-diazo-3-phenyl-2-propanone to give cyclopropane $\mathbf{5 b}$

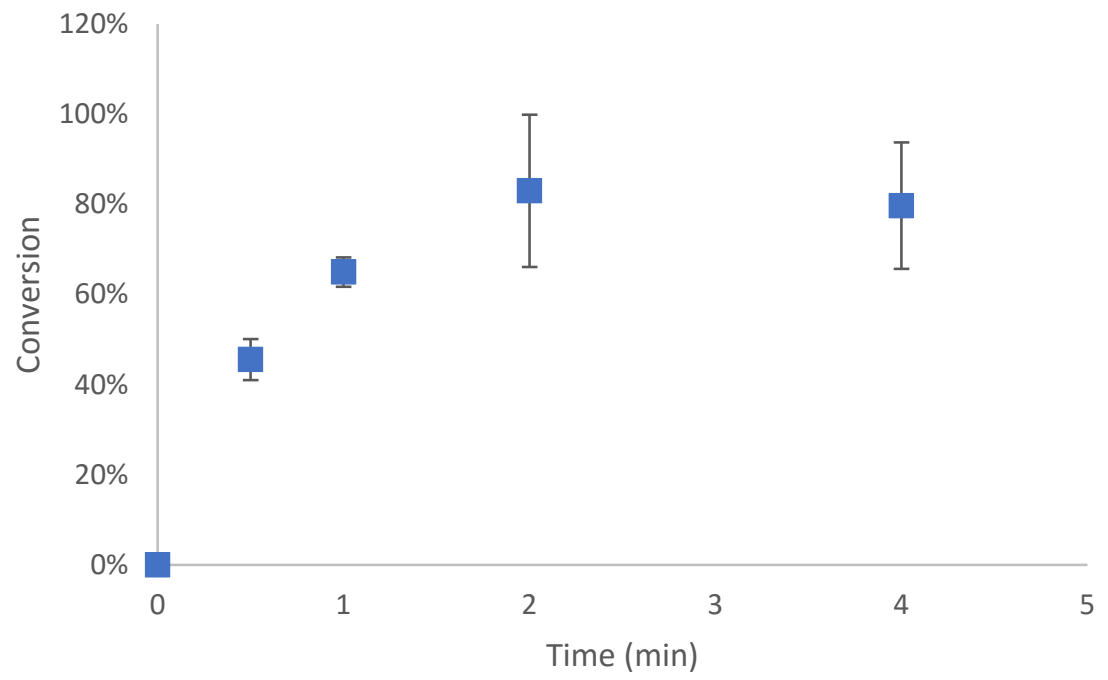

(d) Time-course analysis of Mb(H64G,V68A)-catalyzed cyclopropanation of p-chloro-styrene 4c with 1-diazo-3-phenyl-2-propanone to give cyclopropane $\mathbf{5 c}$ :

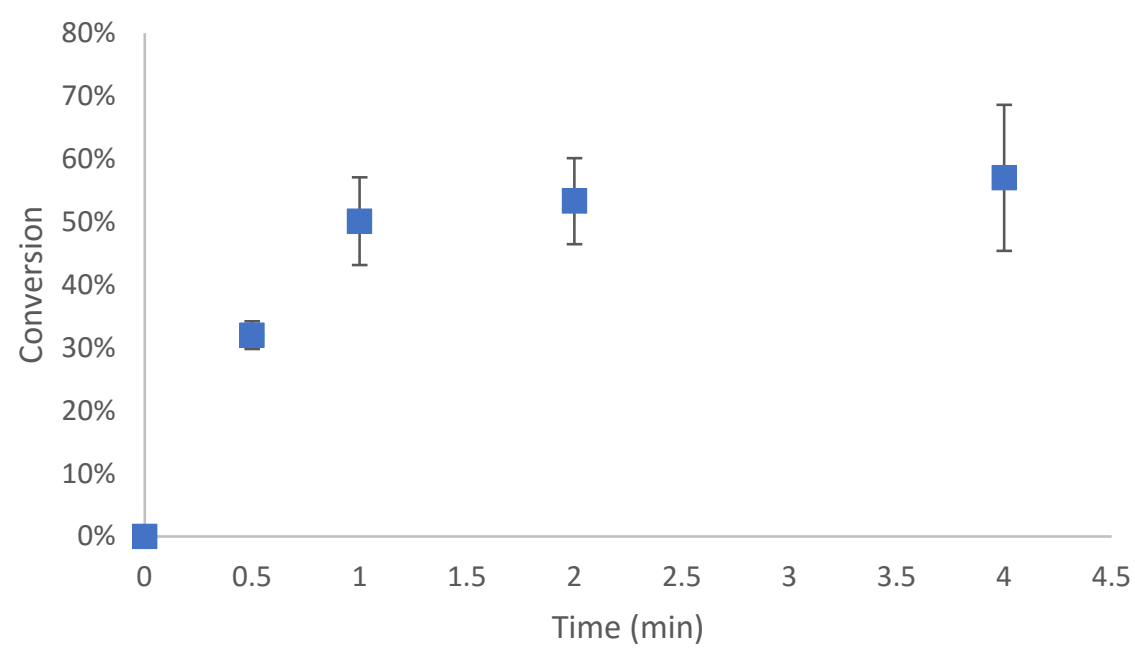


(e) Time-course analysis of $\mathrm{Mb}(\mathrm{H} 64 \mathrm{G}, \mathrm{V} 68 \mathrm{~A})$-catalyzed cyclopropanation of p-bromo-styrene 4d with 1-diazo-3-phenyl-2-propanone to give cyclopropane $\mathbf{5 d}$ :

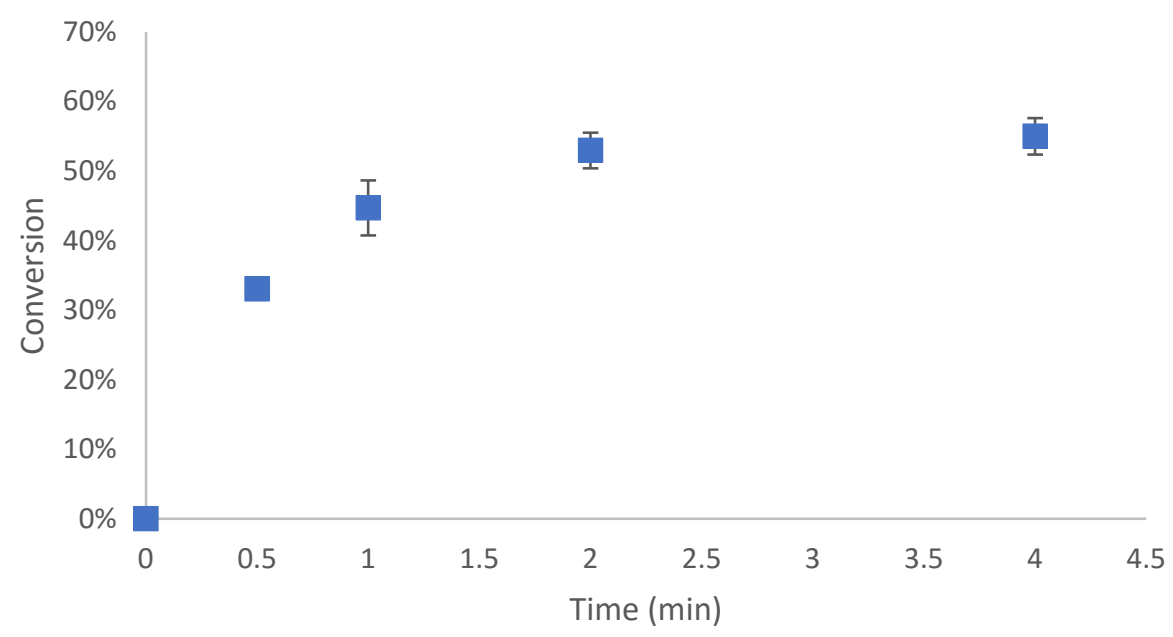


Figure S2. Plots of initial rates of product formation for Mb(H64G,V68A)-catalyzed cyclopropanation of styrene (1) and styrene derivatives 4a-4d in the presence of 1-diazo-3-phenyl2-propanone (2). Reaction conditions: $10 \mu \mathrm{M}$ enzyme, $10 \mathrm{mM}$ styrene 1, $2.5 \mathrm{mM}$ diazoketone 2 , $10 \mathrm{mM} \mathrm{Na} 2 \mathrm{~S}_{2} \mathrm{O}_{4}$ in $50 \mathrm{mM}$ sodium borate buffer ( $\mathrm{pH} 9.0$ ) with $10 \% \mathrm{EtOH}$, room temperature, anaerobic atmosphere. Reactions were quenched with $50 \mu \mathrm{L} \mathrm{3M} \mathrm{HCl}$ at $0.5 \mathrm{~min}$ and $1 \mathrm{~min}$, followed by DCM extraction and chiral SFC analysis.

(a) Initial rate plot of $\mathrm{Mb}(\mathrm{H} 64 \mathrm{G}, \mathrm{V} 68 \mathrm{~A})$-catalyzed cyclopropanation of styrene 1 with 1-diazo-3phenyl-2-propanone 2 to give cyclopropane $\mathbf{3}$ :

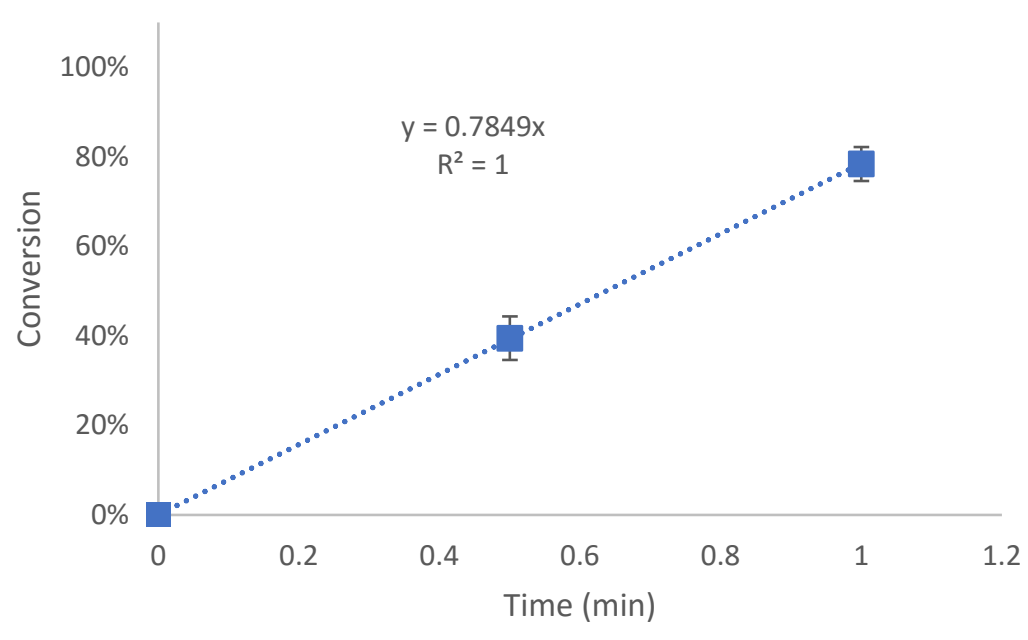

(b) Initial rate plot of $\mathrm{Mb}(\mathrm{H} 64 \mathrm{G}, \mathrm{V} 68 \mathrm{~A})$-catalyzed cyclopropanation of styrene 1 with 1-diazo-3phenyl-2-propanone $\mathbf{2}$ to give cyclopropane $\mathbf{5 a}$ :

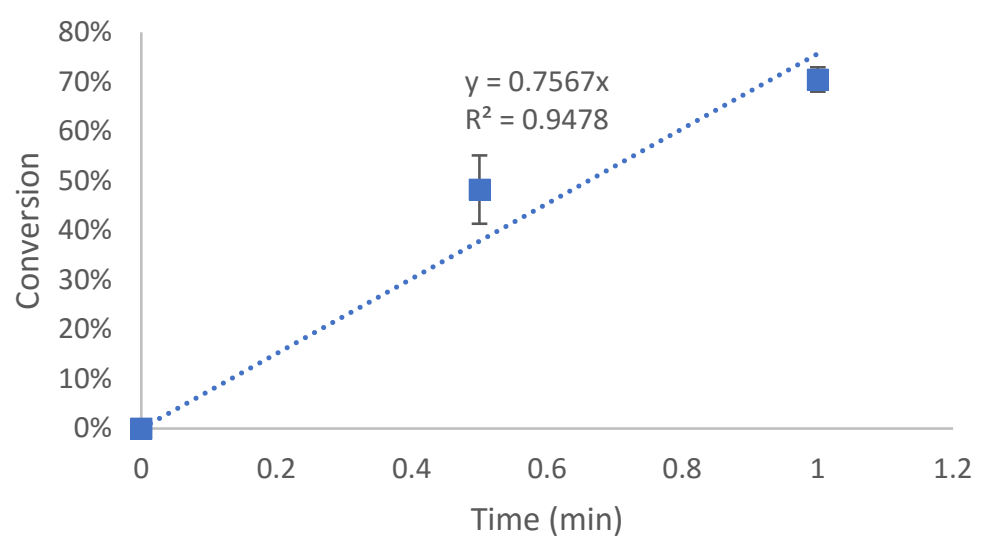


(c) Initial rate plot of $\mathrm{Mb}(\mathrm{H} 64 \mathrm{G}, \mathrm{V} 68 \mathrm{~A})$-catalyzed cyclopropanation of styrene 1 with 1-diazo-3phenyl-2-propanone $\mathbf{2}$ to give cyclopropane $\mathbf{5 b}$ :

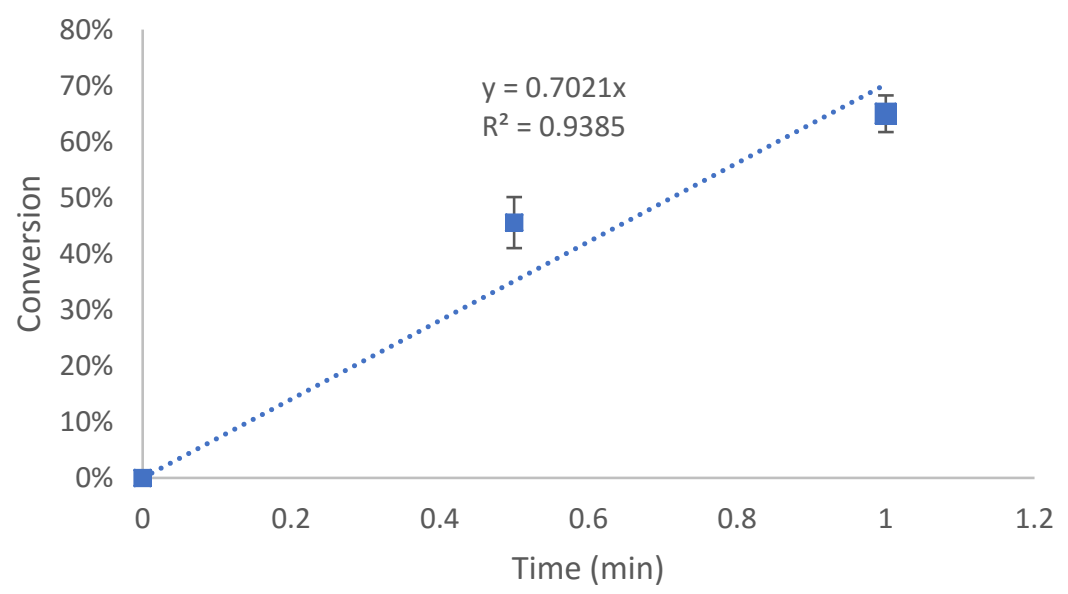

(d) Initial rate plot of $\mathrm{Mb}(\mathrm{H} 64 \mathrm{G}, \mathrm{V} 68 \mathrm{~A})$-catalyzed cyclopropanation of styrene 1 with 1-diazo-3phenyl-2-propanone 2 to give cyclopropane $\mathbf{5 c}$ :

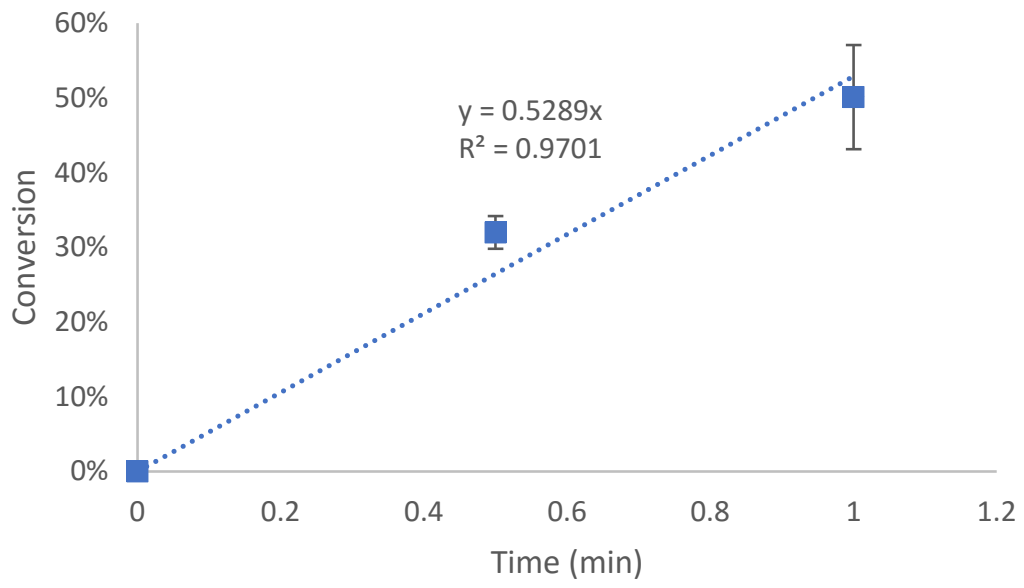


(e) Initial rate plot of $\mathrm{Mb}(\mathrm{H} 64 \mathrm{G}, \mathrm{V} 68 \mathrm{~A})$-catalyzed cyclopropanation of styrene 1 with 1-diazo-3phenyl-2-propanone $\mathbf{2}$ to give cyclopropane $\mathbf{5 d}$ :

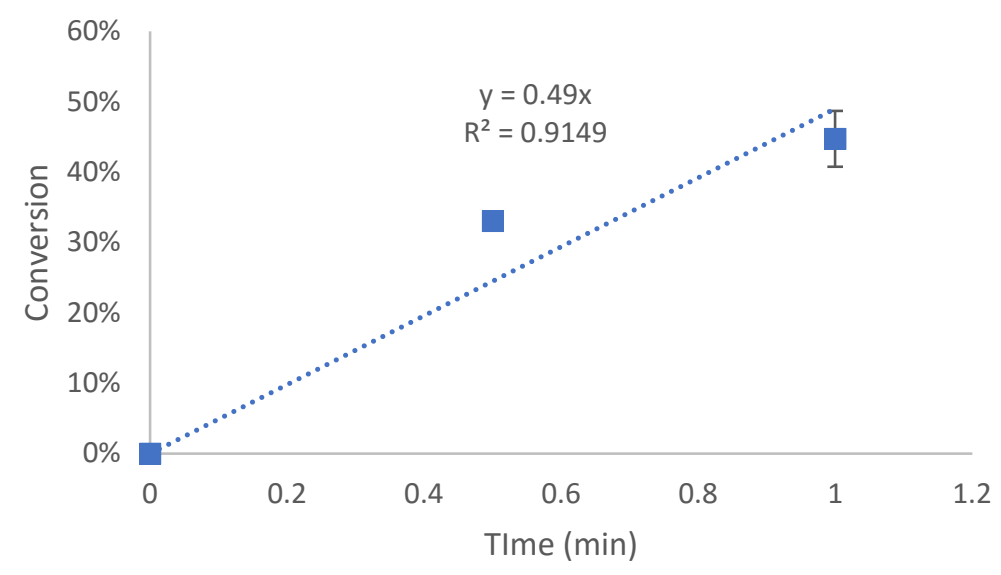

\begin{tabular}{cccc}
\hline Entry & Substrate & Product & $\begin{array}{c}\text { Product Formation Rate } \\
\text { (TON/min) }\end{array}$ \\
\hline 1 & Styrene & $\mathbf{3}$ & $196 \pm 10$ \\
2 & 4-Me-Styrene & $\mathbf{5 a}$ & $190 \pm 17$ \\
3 & 4-MeO-Styrene & $\mathbf{5 b}$ & $176 \pm 14$ \\
4 & 4-Cl-Styrene & $\mathbf{5 c}$ & $132 \pm 13$ \\
5 & 4-Br-Styrene & $\mathbf{5 d}$ & $123 \pm 7$ \\
\hline
\end{tabular}


Figure S3. Chiral SFC, GC, or HPLC chromatograms for determination of enantiomeric and diastereomeric excess in the $\mathrm{Mb}(\mathrm{H} 64 \mathrm{G}, \mathrm{V} 68 \mathrm{~A})$-catalyzed cyclopropanation reaction with olefins $(\mathbf{1}, \mathbf{4 a}-\mathbf{4 k})$ and diazoketones $(\mathbf{2}, \mathbf{6 a}-\mathbf{6 n})$. Reference racemic samples were prepared with $\mathrm{Fe}(\mathrm{TPP}) \mathrm{Cl}$ as described in the experimental procedures.

(a) Reaction with styrene $\mathbf{1}$ and 1-diazo-3-phenyl-2-propanone $\mathbf{2}$ to give cyclopropane $\mathbf{3}$ :

i. SFC analysis of racemic product 3 ( 2 trans enantiomers + unresolved cis isomers):

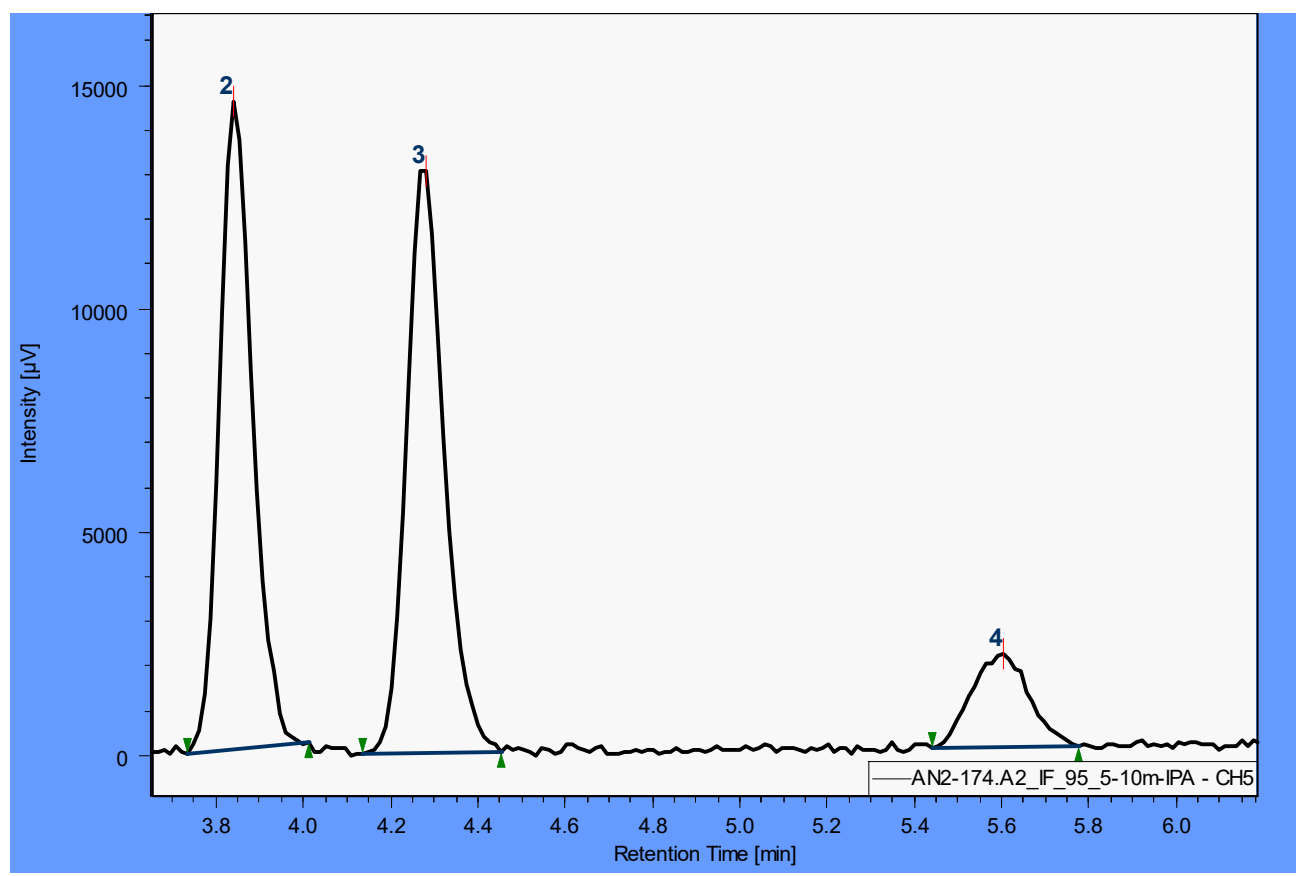

ii. SFC analysis of $\mathrm{Mb}(\mathrm{H} 64 \mathrm{G}, \mathrm{V} 68 \mathrm{~A})$-catalyzed reaction:

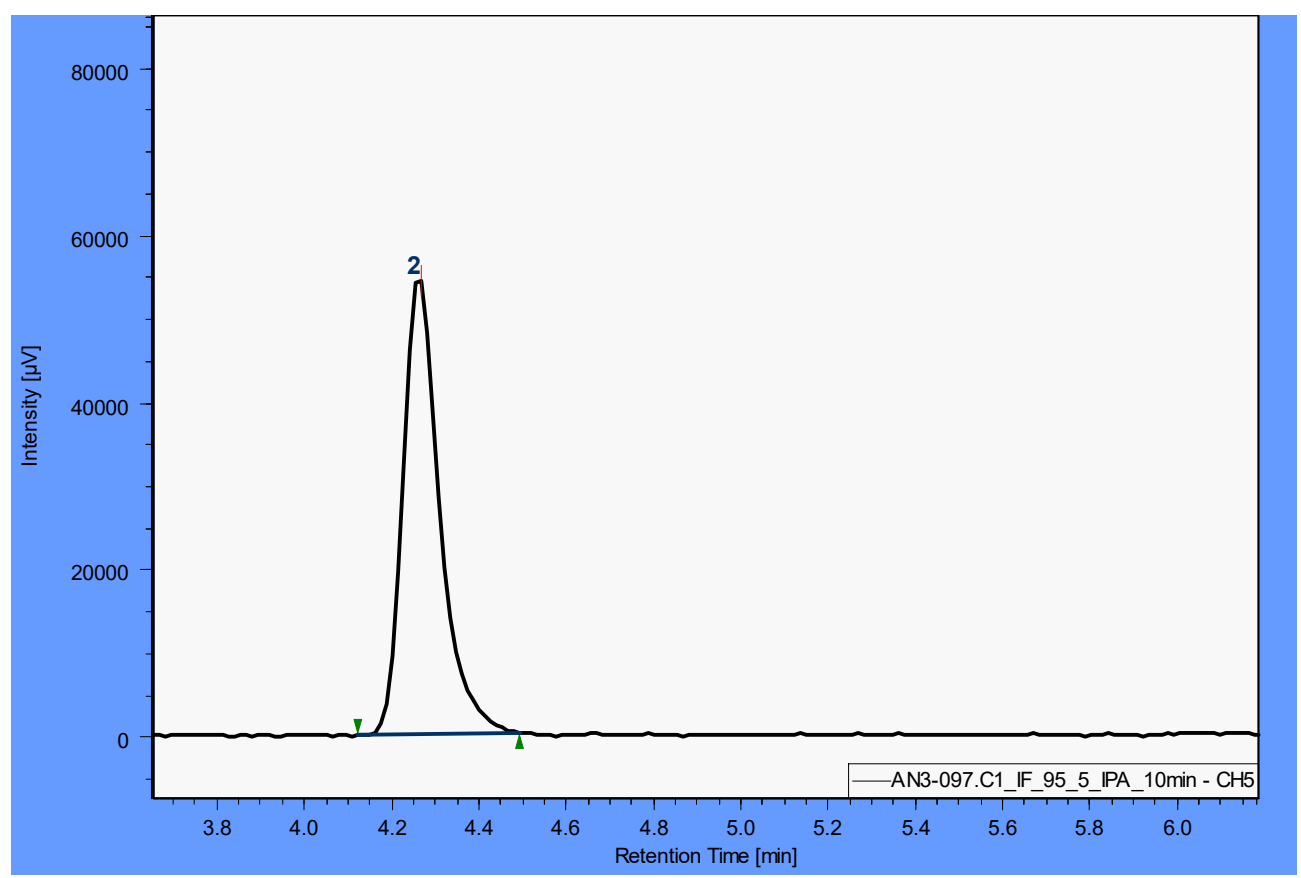


(b) Reaction with 4-methylstyrene 4a and diazoketone $\mathbf{2}$ to give cyclopropane 5a:

i. SFC analysis of racemic product $4 \mathbf{4}$ ( 2 trans enantiomers +2 cis enantiomers):

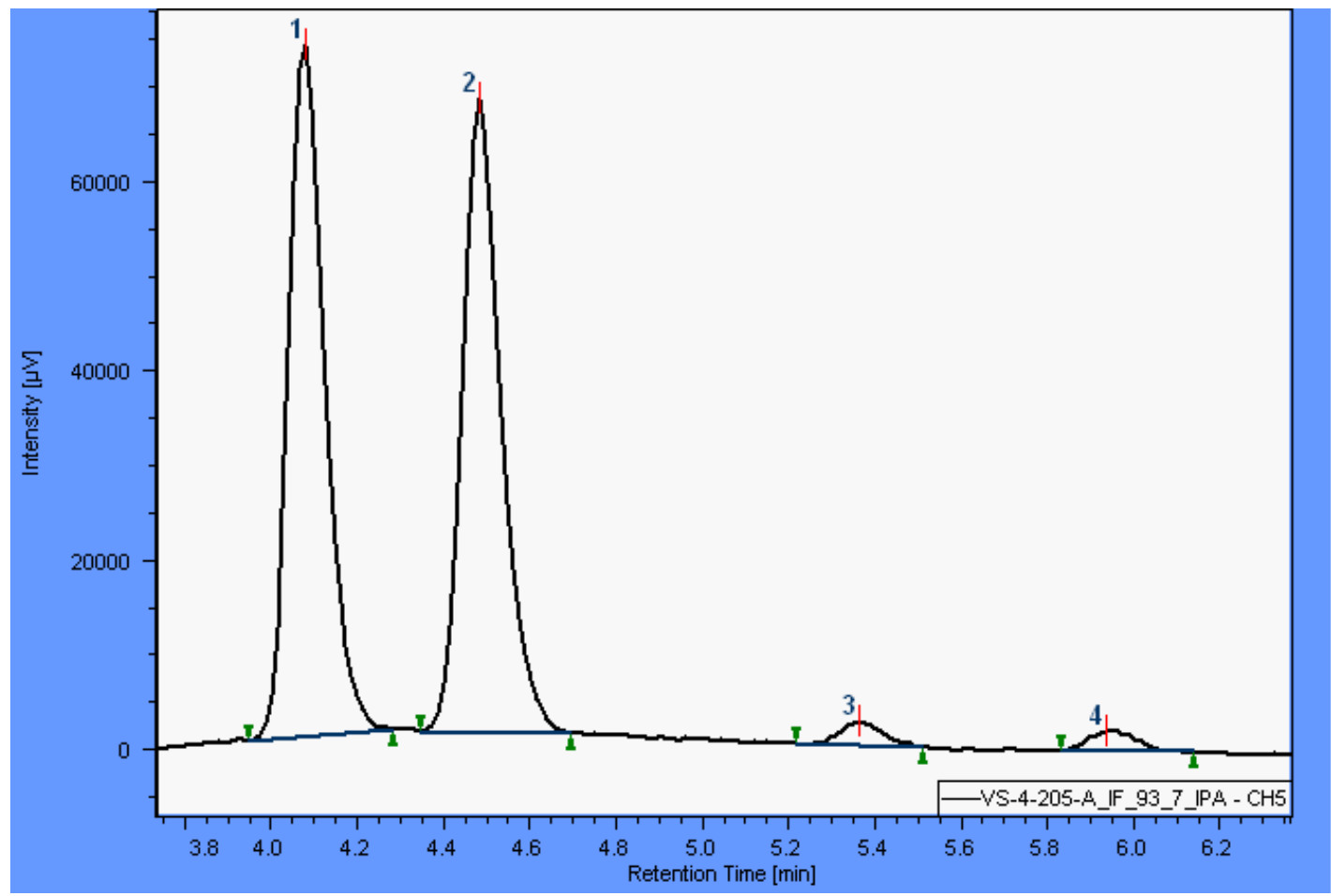

ii. SFC analysis of $\mathrm{Mb}(\mathrm{H} 64 \mathrm{G}, \mathrm{V} 68 \mathrm{~A})$-catalyzed reaction:

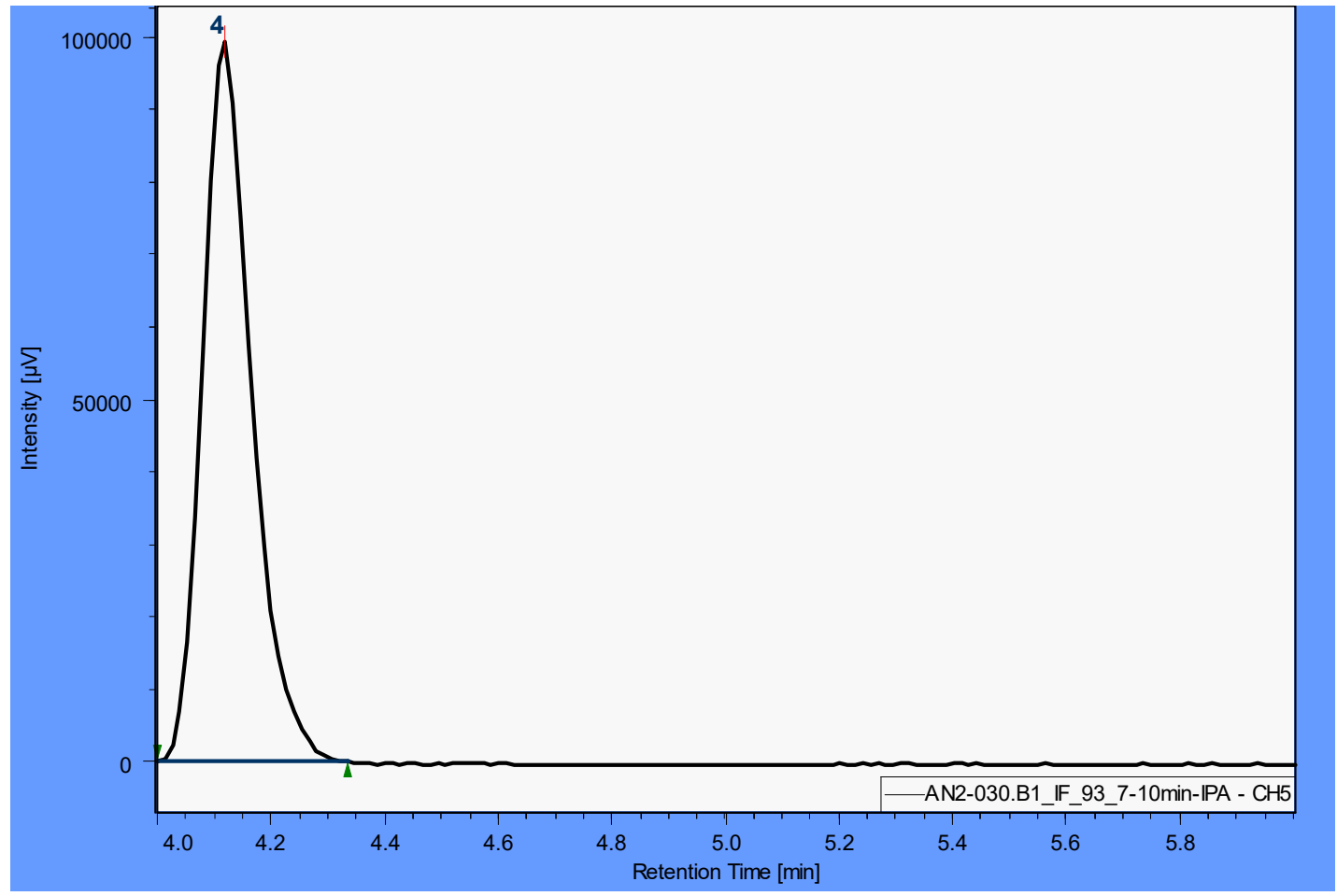


(c) Reaction with 4-methoxystyrene $\mathbf{4 b}$ and diazoketone $\mathbf{2}$ to give cyclopropane $\mathbf{5 b}$ :

i. SFC analysis of racemic product $\mathbf{4 b}$ ( 2 trans enantiomers +2 cis enantiomers):

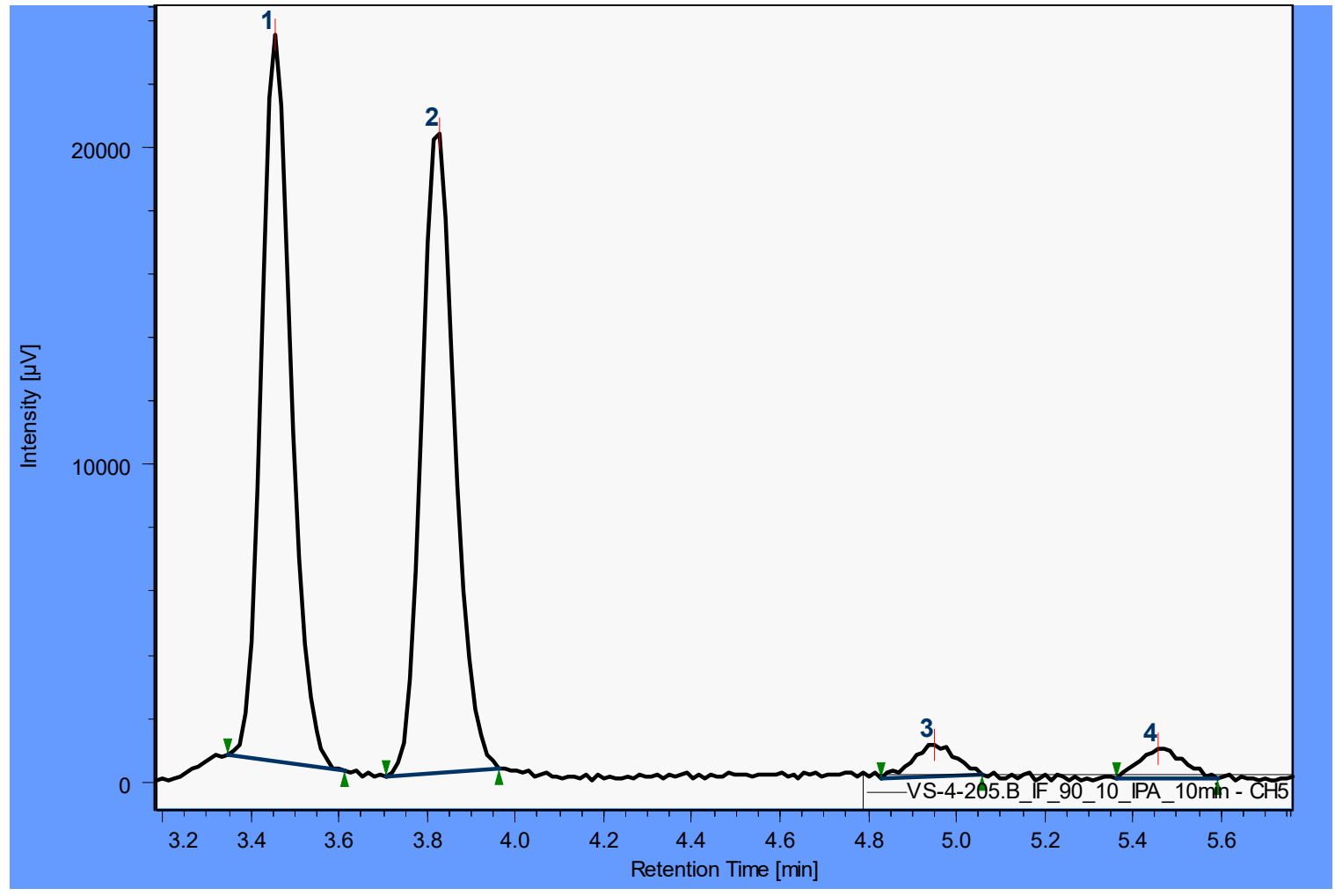

ii. SFC analysis of $\mathrm{Mb}(\mathrm{H} 64 \mathrm{G}, \mathrm{V} 68 \mathrm{~A})$-catalyzed reaction:

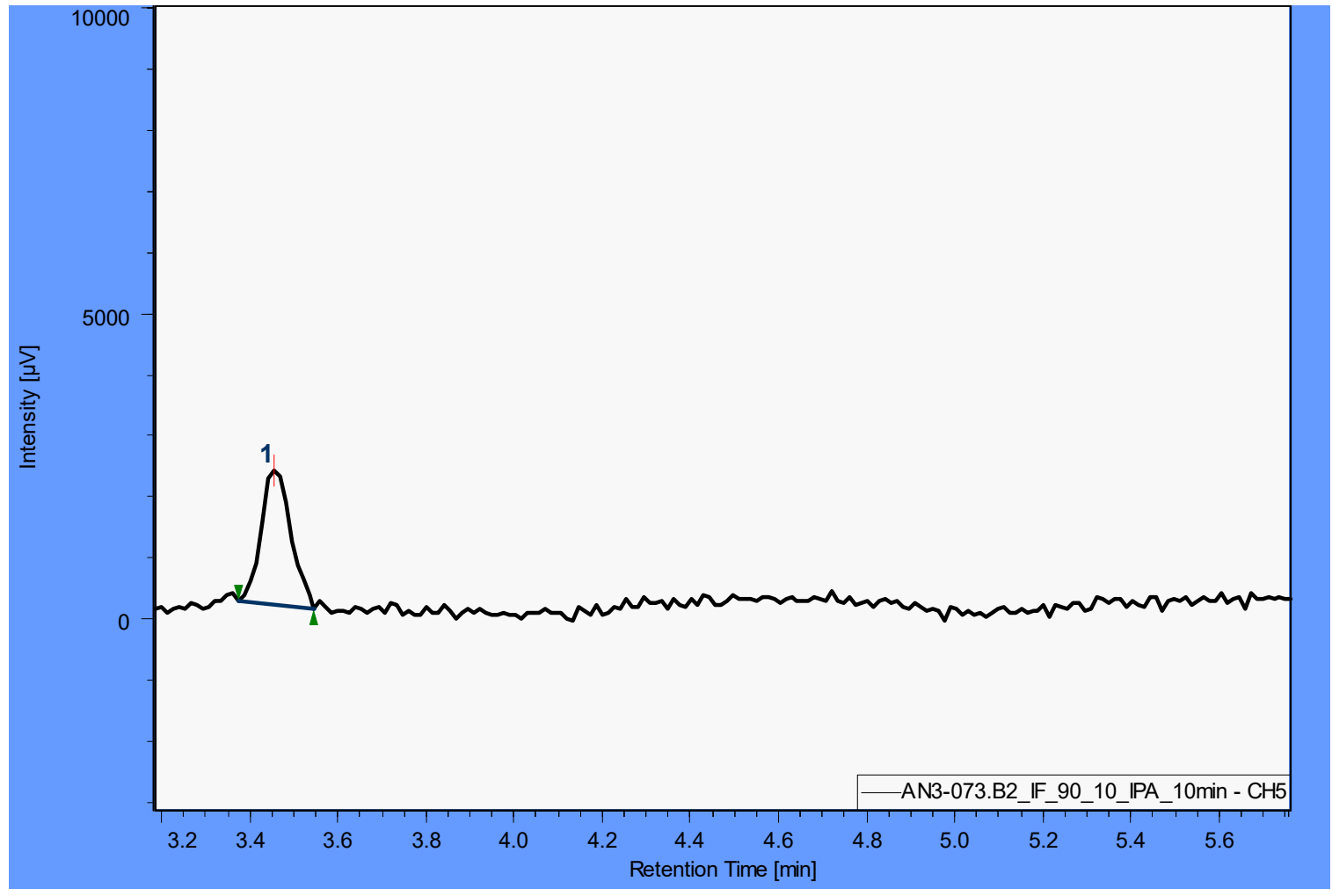


(d) Reaction with 4-chlorostyrene $4 \mathbf{c}$ and diazoketone $\mathbf{2}$ to give cyclopropane $\mathbf{5 c}$ :

i. SFC analysis of racemic product $4 \mathbf{c}$ ( 2 trans enantiomers +2 cis enantiomers):

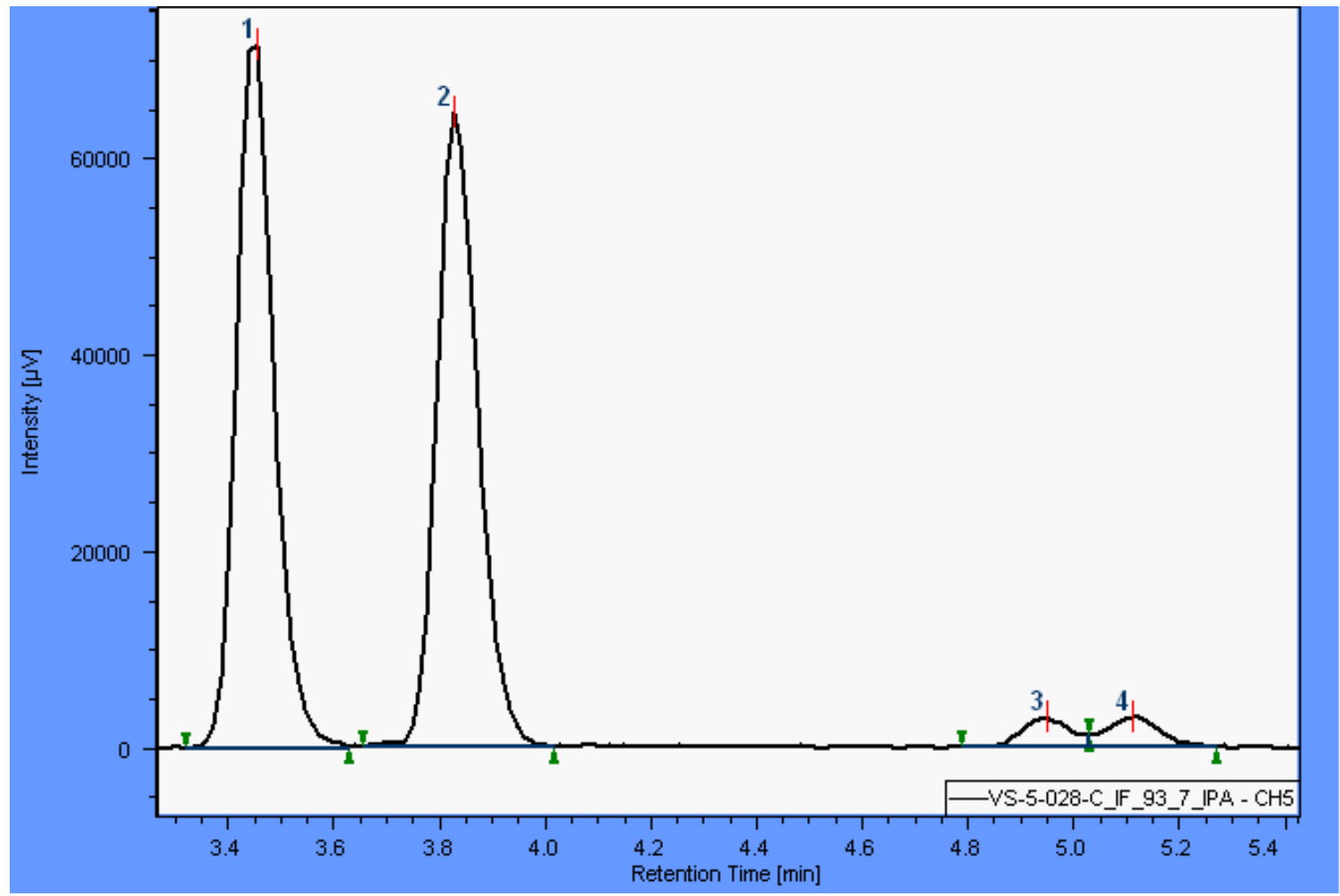

ii. SFC analysis of $\mathrm{Mb}(\mathrm{H} 64 \mathrm{G}, \mathrm{V} 68 \mathrm{~A})$-catalyzed reaction:

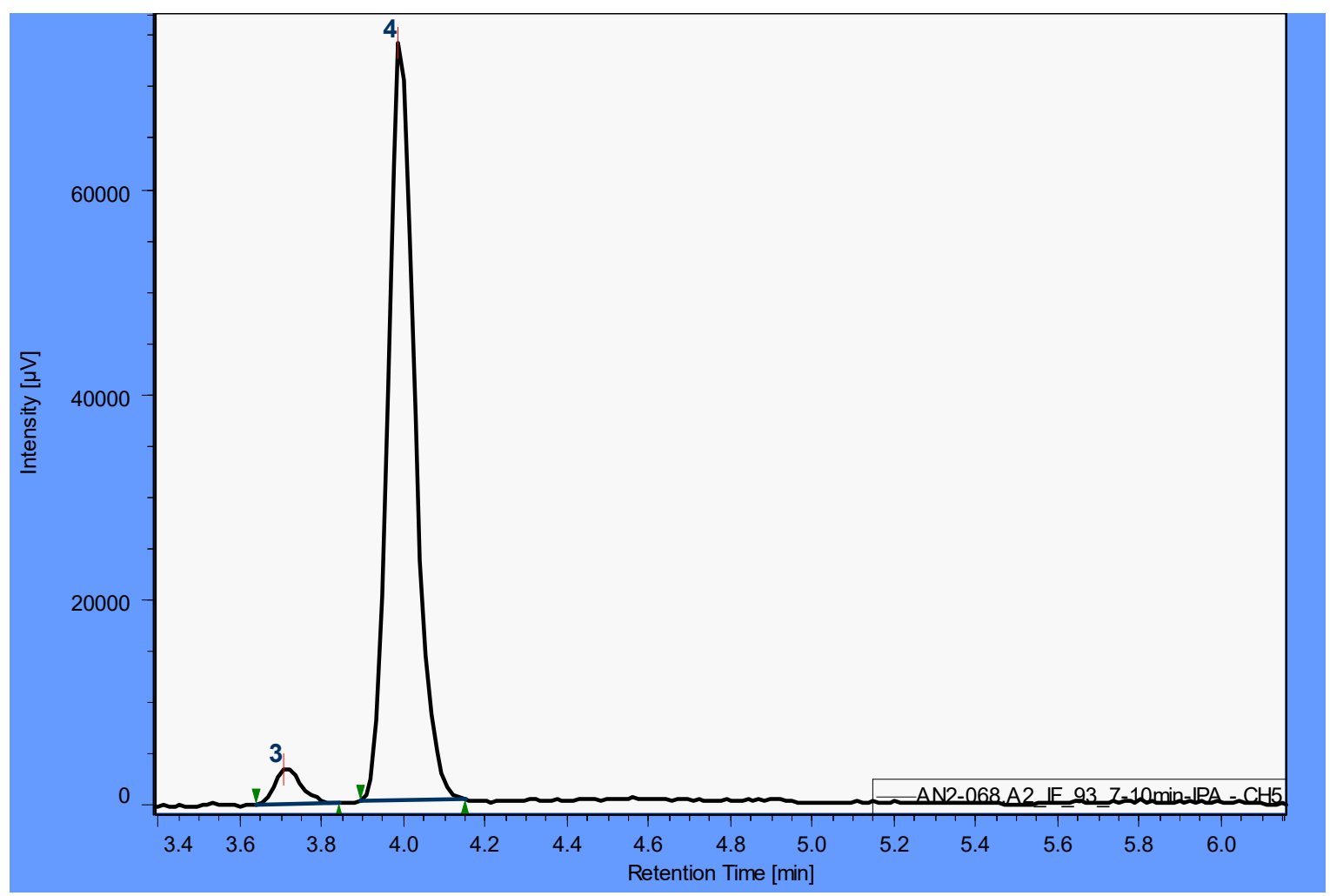


(e) Reaction with 4-bromostyrene $\mathbf{4 d}$ and diazoketone $\mathbf{2}$ to give cyclopropane $\mathbf{5 d}$ :

i. SFC analysis of racemic product $\mathbf{4 d}$ (unresolved cis enantiomers +2 trans enantiomers):

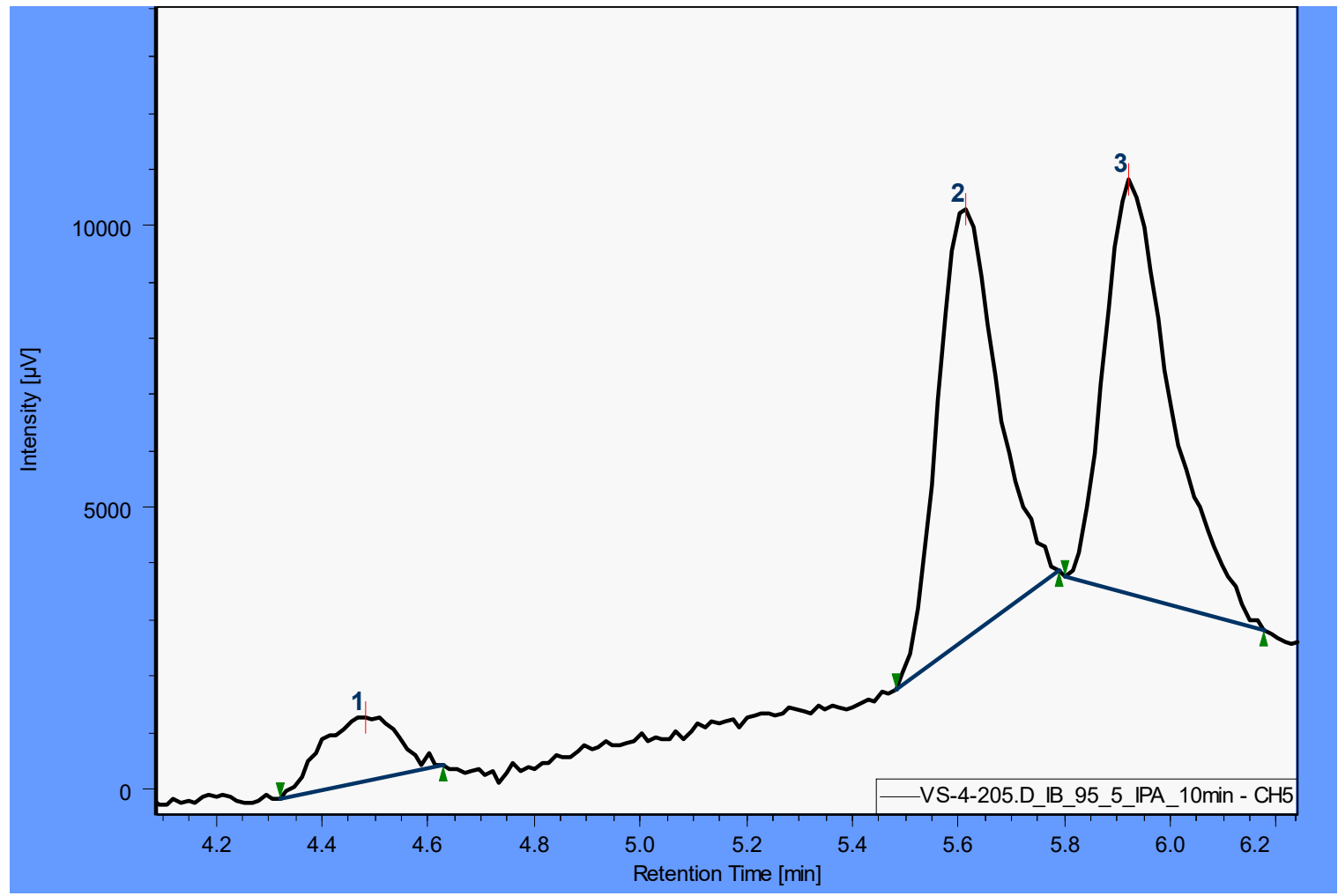

SFC analysis of $\mathrm{Mb}(\mathrm{H} 64 \mathrm{G}, \mathrm{V} 68 \mathrm{~A})$-catalyzed reaction:

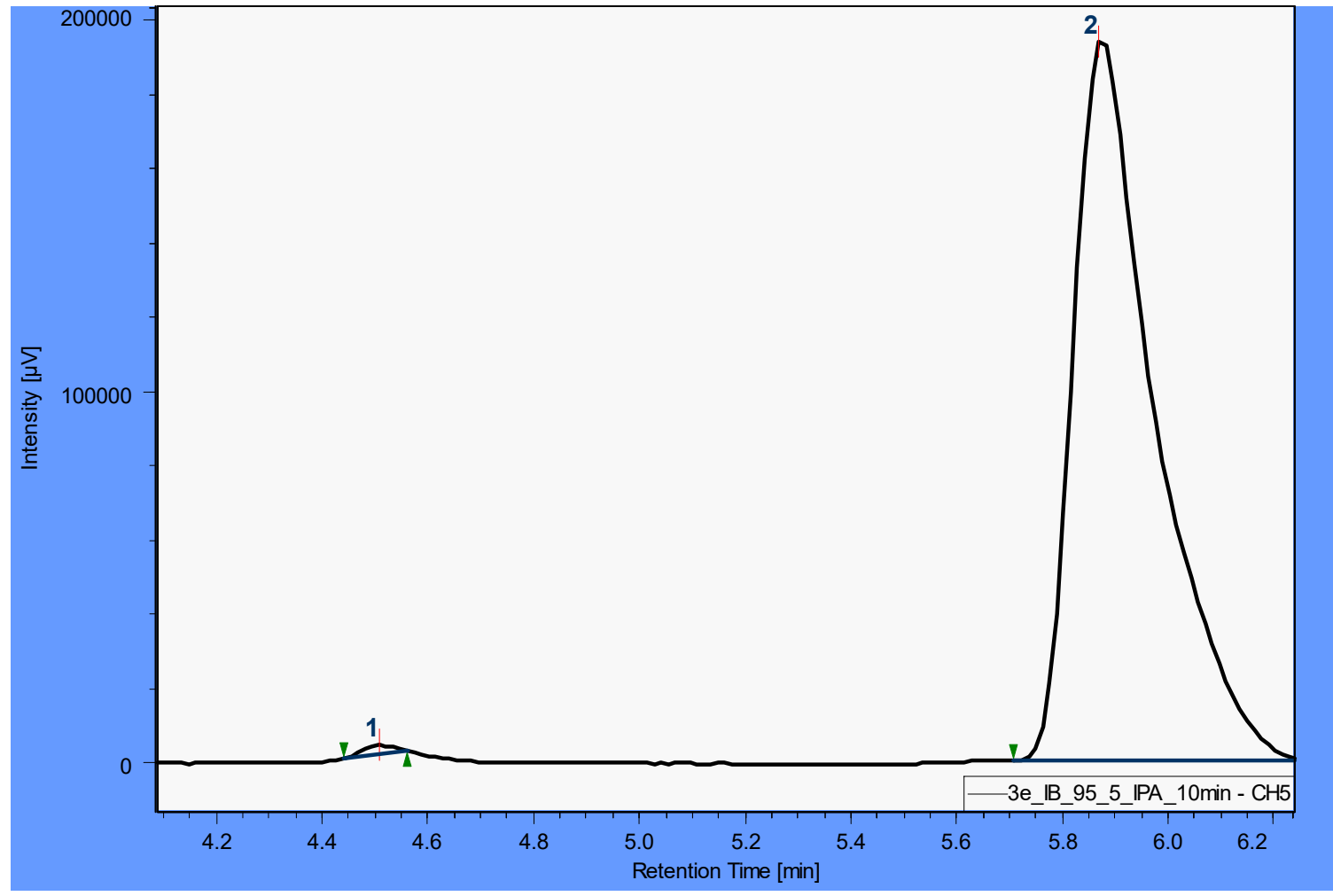


(f) Reaction with 4-trifluoromethylstyrene $4 \mathbf{e}$ and diazoketone 2 to give cyclopropane $5 \mathrm{e}$ :

i. SFC analysis of racemic product $4 \mathbf{e}$ ( 2 trans enantiomers +2 cis enantiomers):

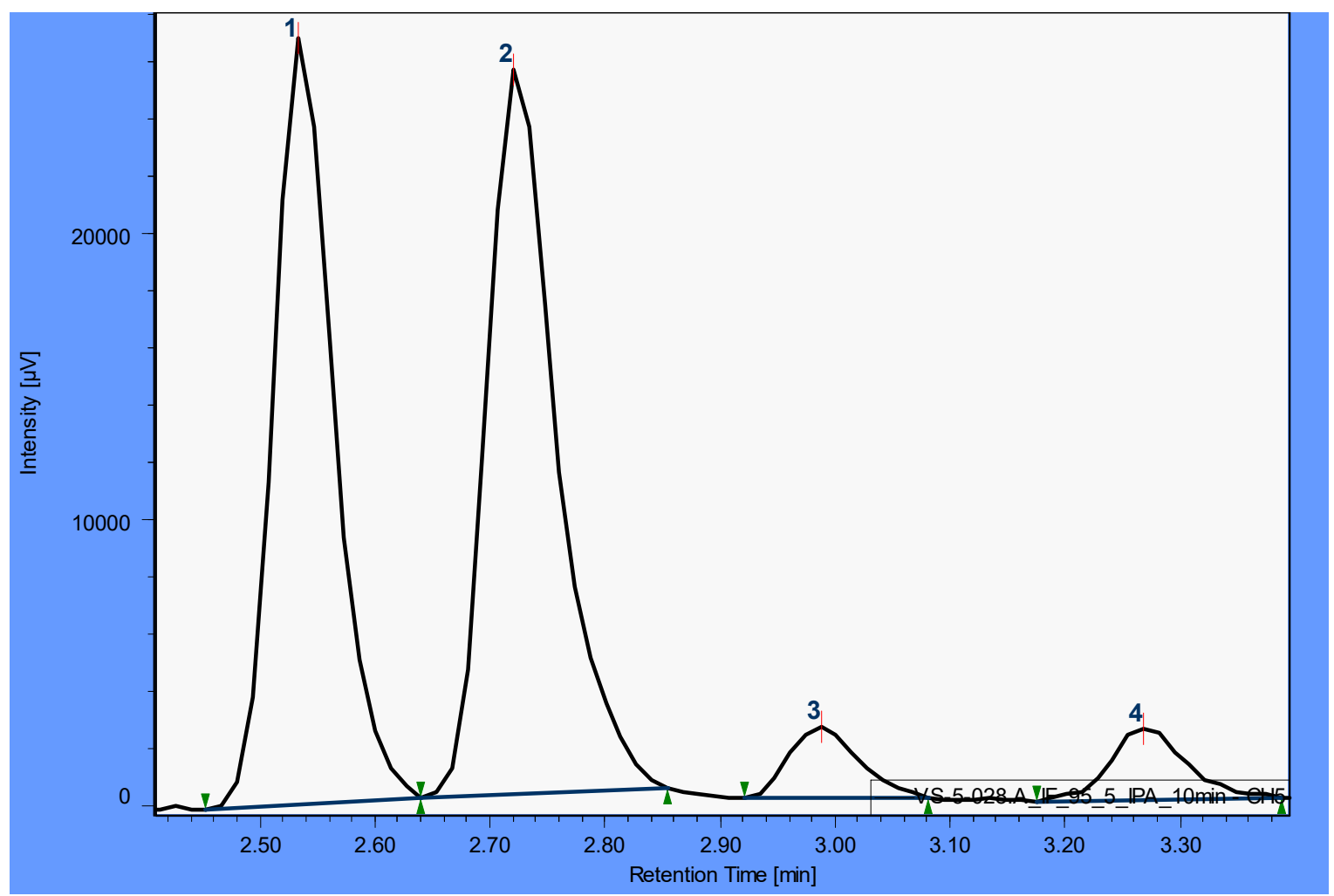

ii. SFC analysis of $\mathrm{Mb}(\mathrm{H} 64 \mathrm{G}, \mathrm{V} 68 \mathrm{~A})$-catalyzed reaction:

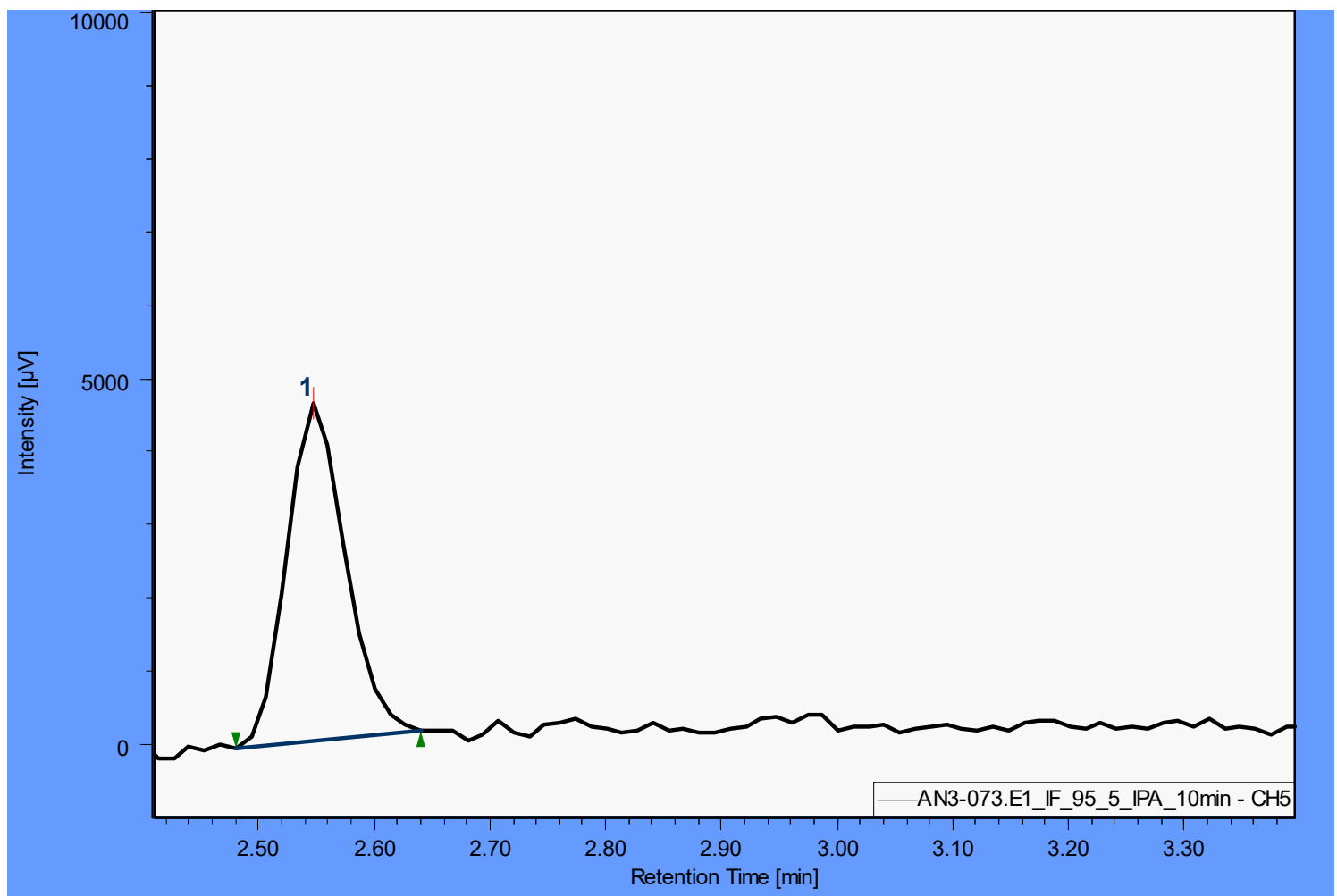


(g) Reaction with 3-methylstyrene $\mathbf{4 f}$ and diazoketone $\mathbf{2}$ to give cyclopropane $\mathbf{5 f}$ :

i. SFC analysis of racemic product $\mathbf{4 f}$ ( 2 trans enantiomers +2 cis enantiomers):

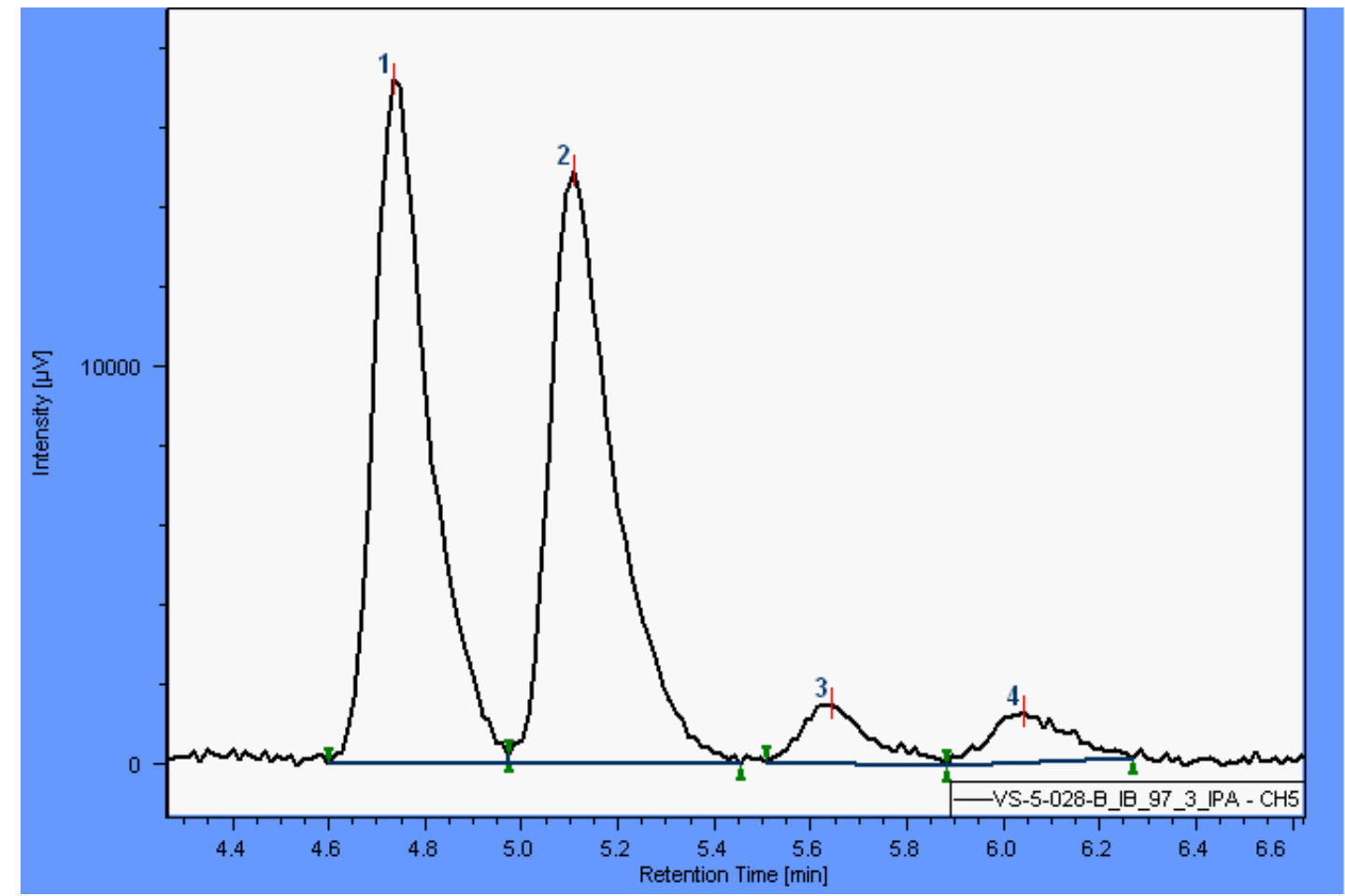

ii. SFC analysis of $\mathrm{Mb}(\mathrm{H} 64 \mathrm{G}, \mathrm{V} 68 \mathrm{~A})$-catalyzed reaction:

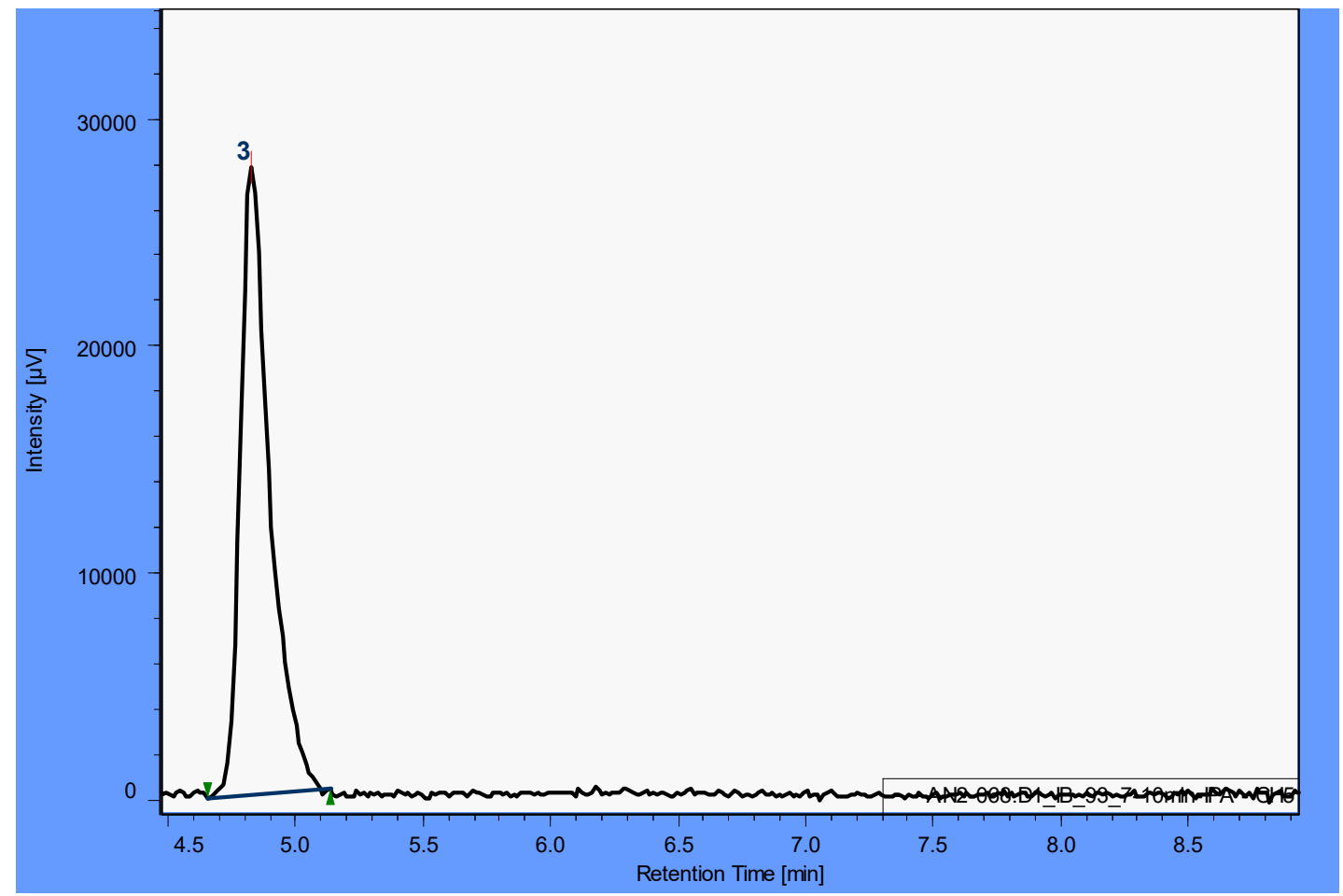


(h) Reaction with 2-methylstyrene $\mathbf{4 g}$ and diazoketone $\mathbf{2}$ to give cyclopropane $\mathbf{5 g}$ :

i. SFC analysis of racemic product $\mathbf{4 g}$ ( 2 trans enantiomers +2 cis enantiomers):

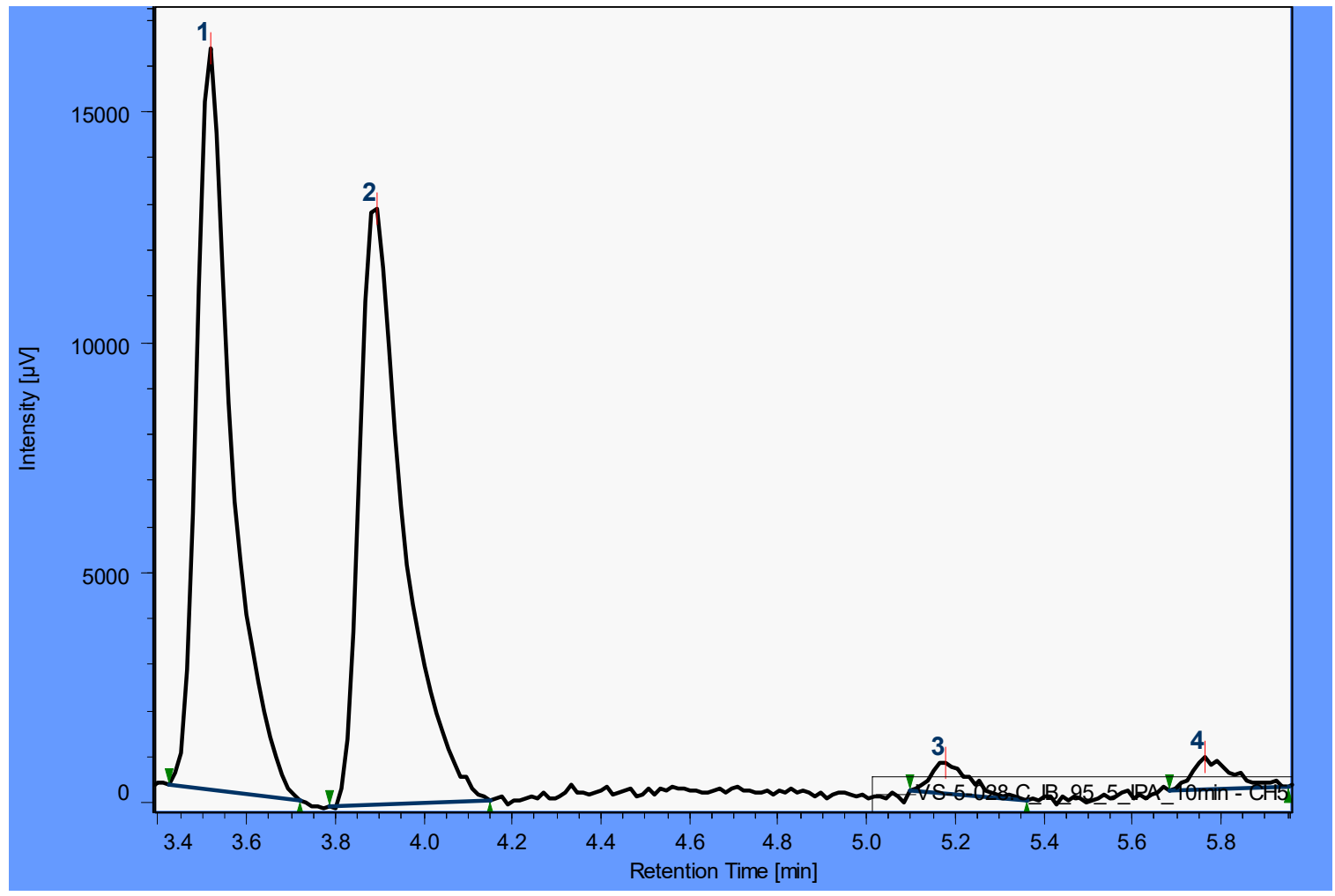

ii. $\mathrm{SFC}$ analysis of $\mathrm{Mb}(\mathrm{H} 64 \mathrm{G}, \mathrm{V} 68 \mathrm{~A})$-catalyzed reaction:

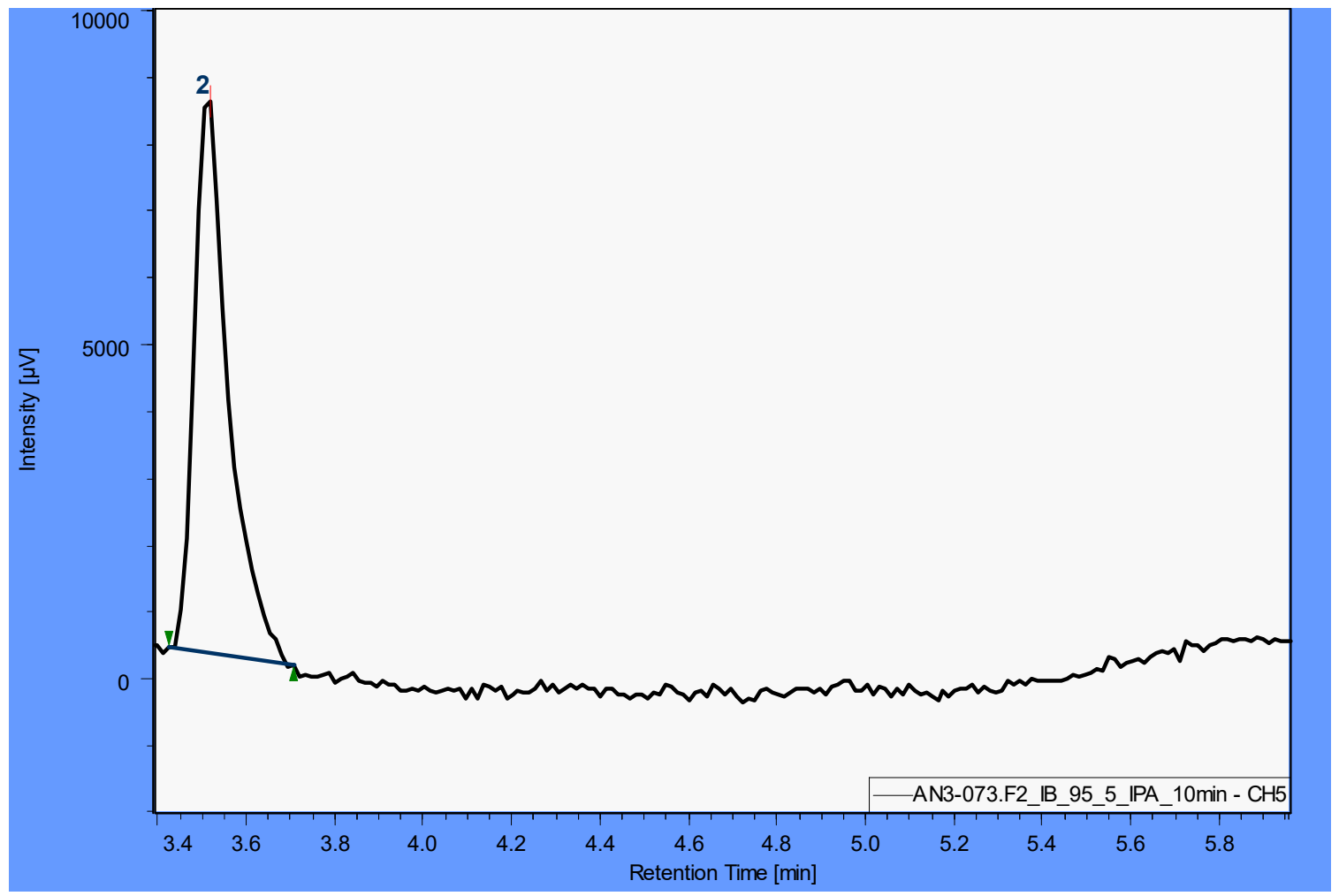


(i) Reaction with $\alpha$-methylstyrene $\mathbf{4 h}$ and diazoketone $\mathbf{2}$ to give cyclopropane $\mathbf{5 h}$ :

i. SFC analysis of racemic product 4 h ( 2 trans enantiomers +2 cis enantiomers):

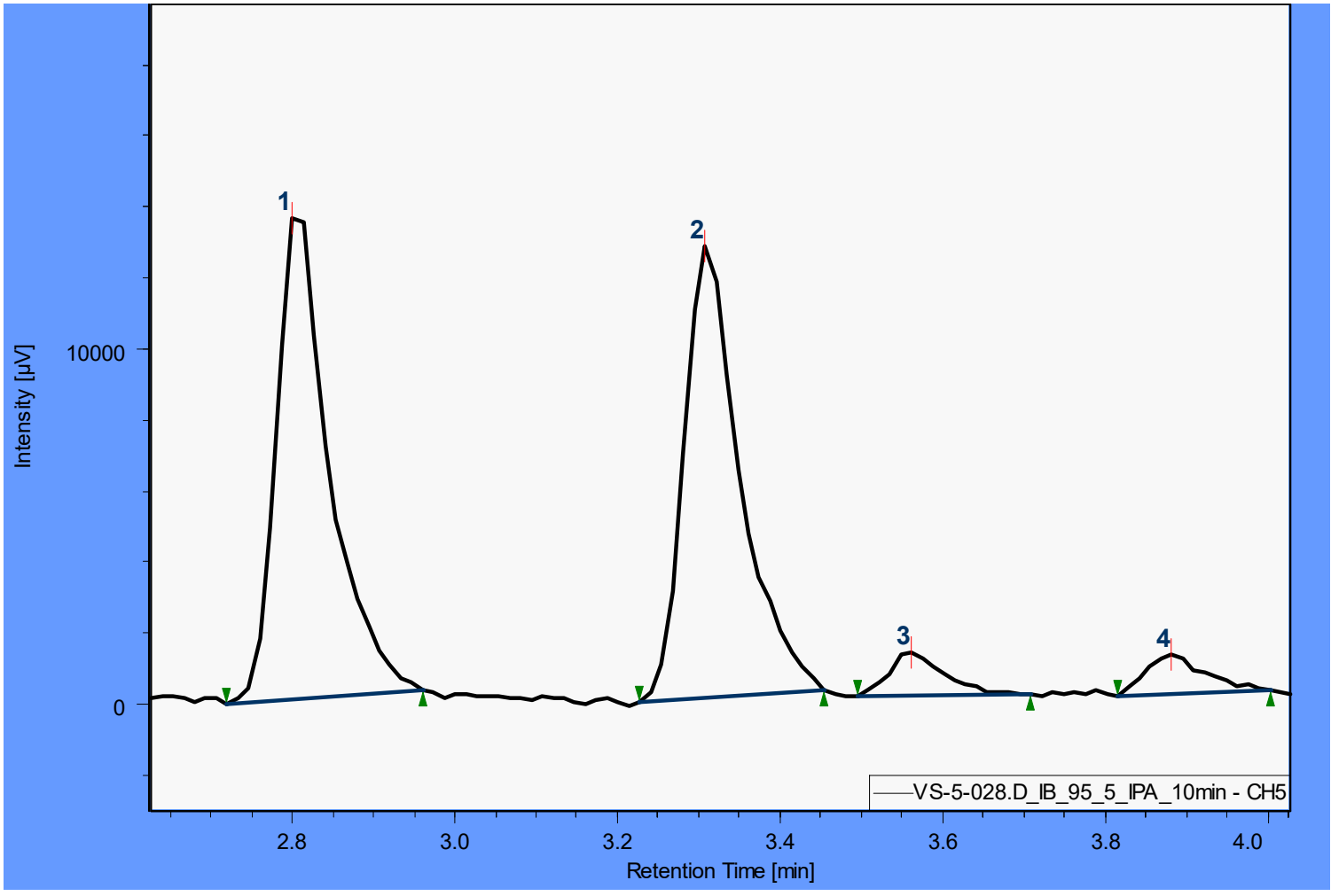

ii. SFC analysis of $\mathrm{Mb}(\mathrm{H} 64 \mathrm{G}, \mathrm{V} 68 \mathrm{~A})$-catalyzed reaction:

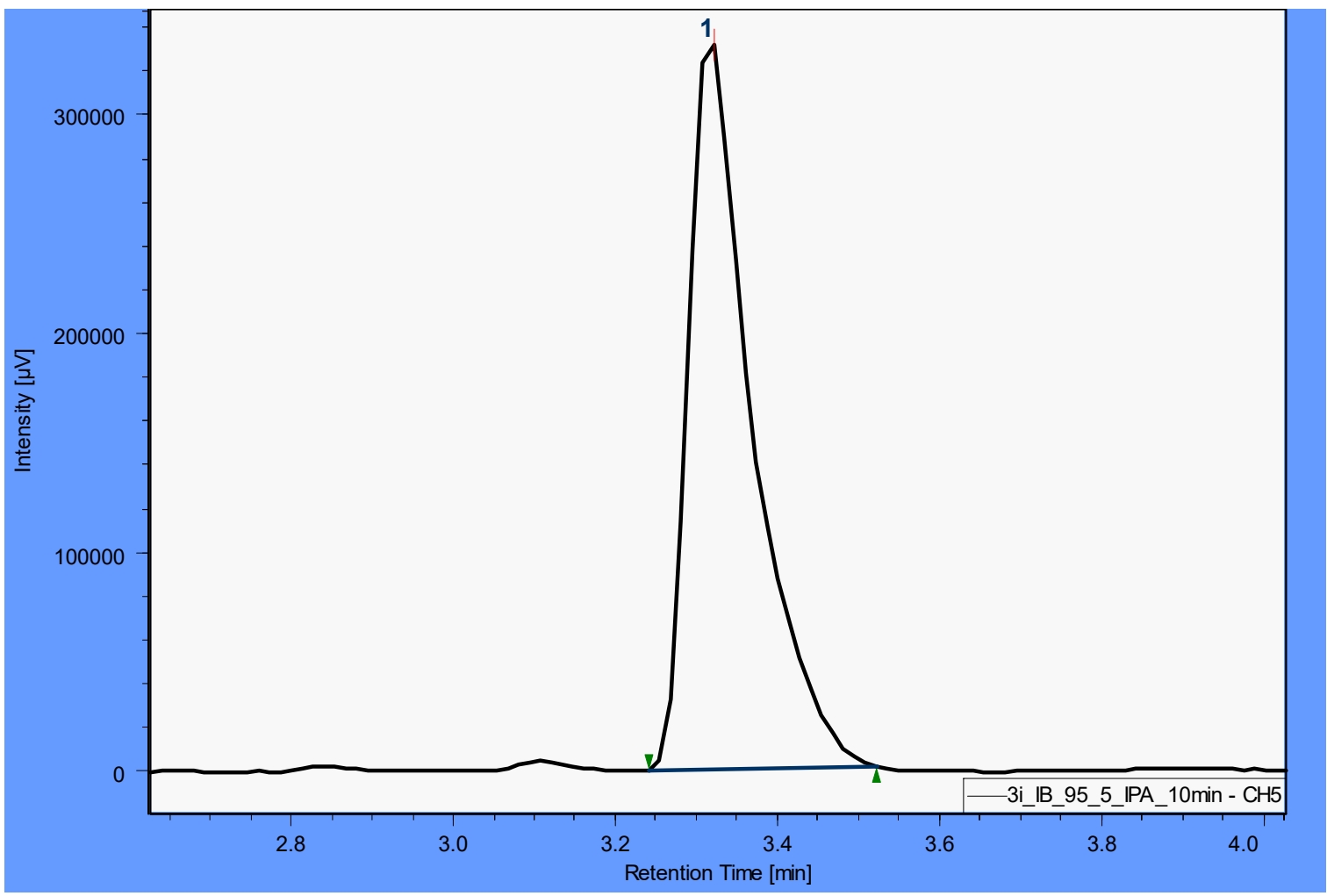


(j) Reaction with 2,3,4,5,6-pentafluorostyrene $4 \mathbf{i}$ and diazoketone 2 to give cyclopropane $\mathbf{5 i}$ :

i. SFC analysis of racemic product $4 \mathbf{i}$ ( 2 trans enantiomers +2 cis enantiomers):

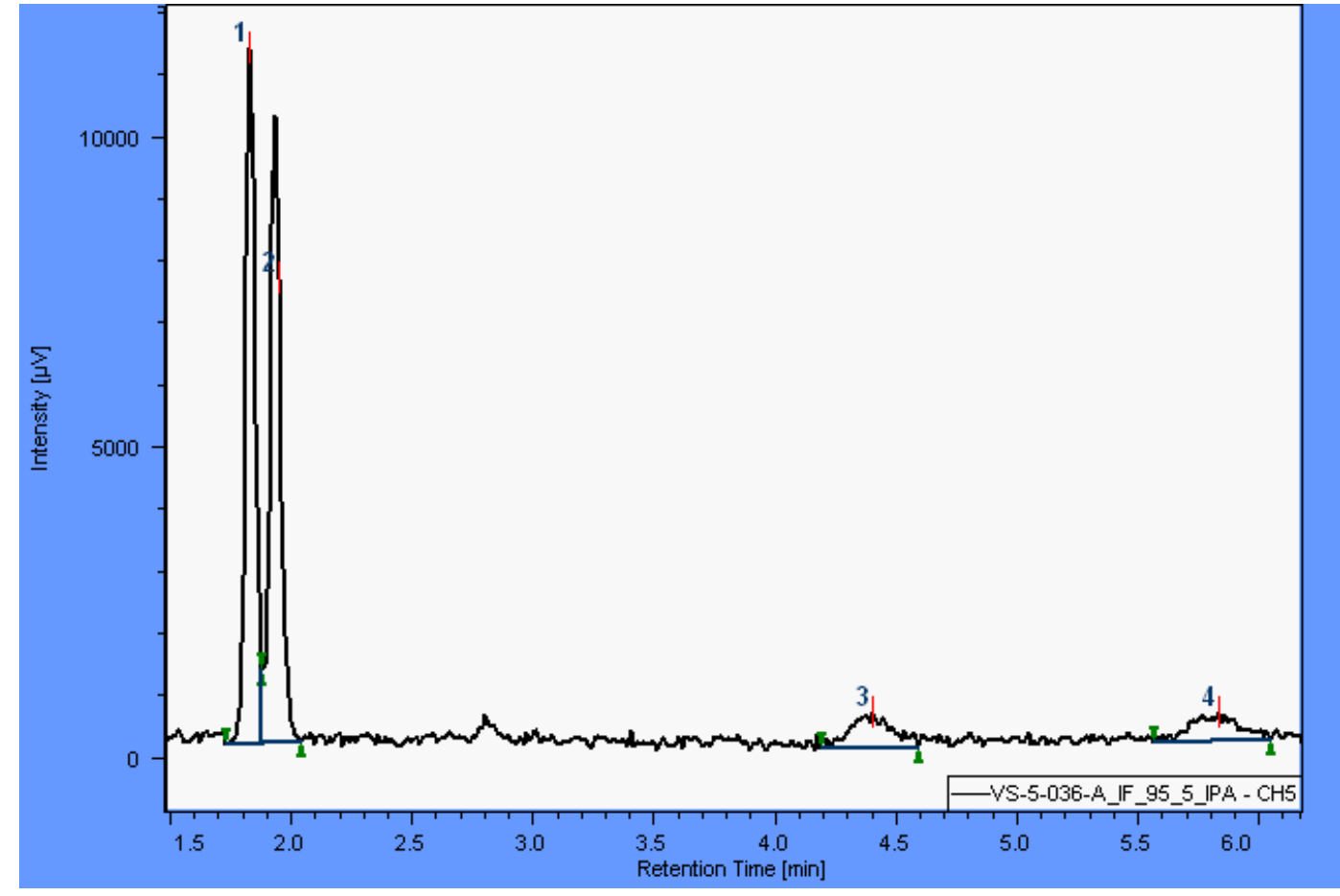

ii. $\mathrm{SFC}$ analysis of $\mathrm{Mb}(\mathrm{H} 64 \mathrm{G}, \mathrm{V} 68 \mathrm{~A})$-catalyzed reaction:

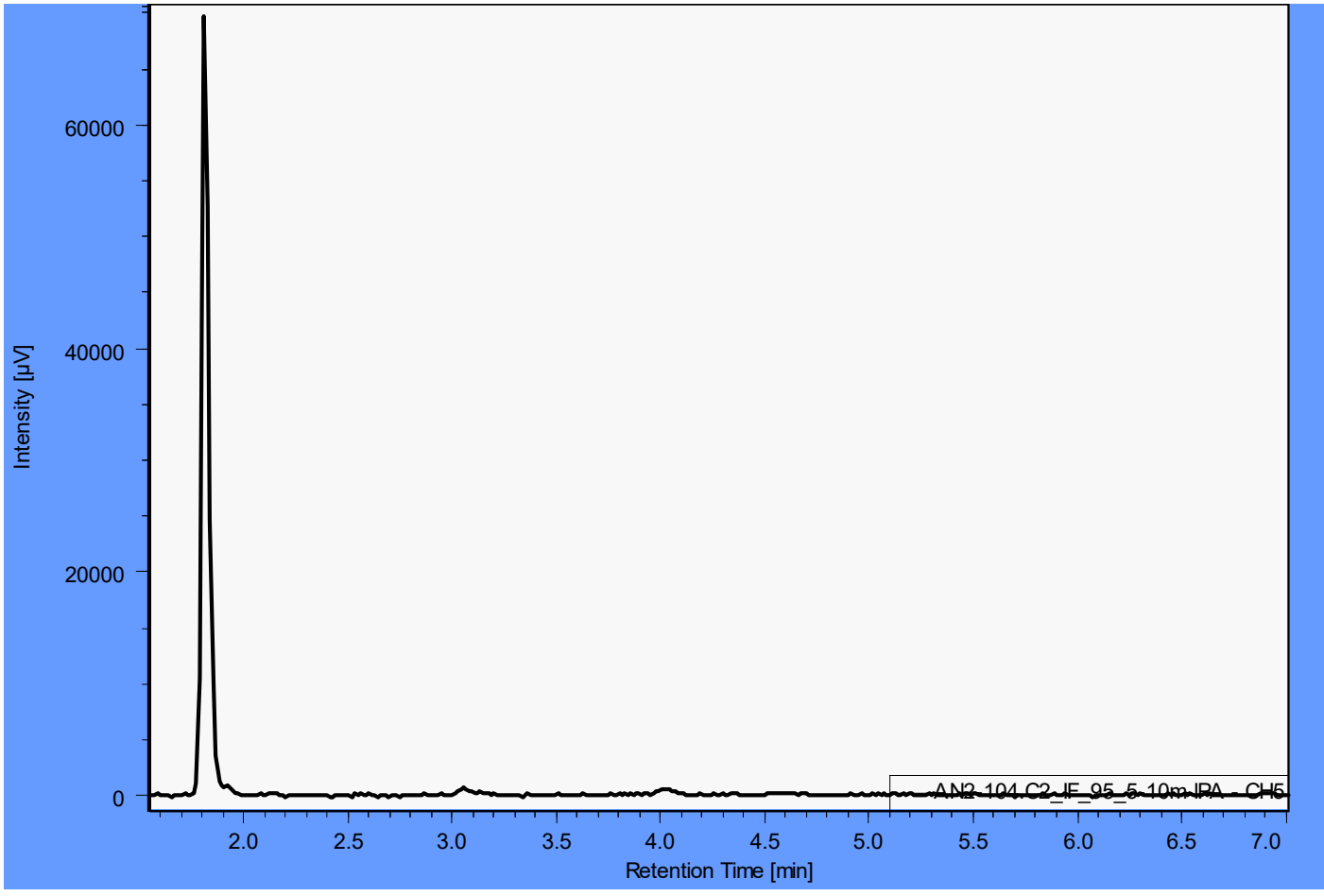


(k) Reaction with 3-(prop-1-en-2-yl)thiophene $\mathbf{4 j}$ and diazoketone $\mathbf{2}$ to give cyclopropane $\mathbf{5 j}$ :

i. SFC analysis of racemic product $\mathbf{4 j}$ (unresolved cis enantiomers +2 trans enantiomers):

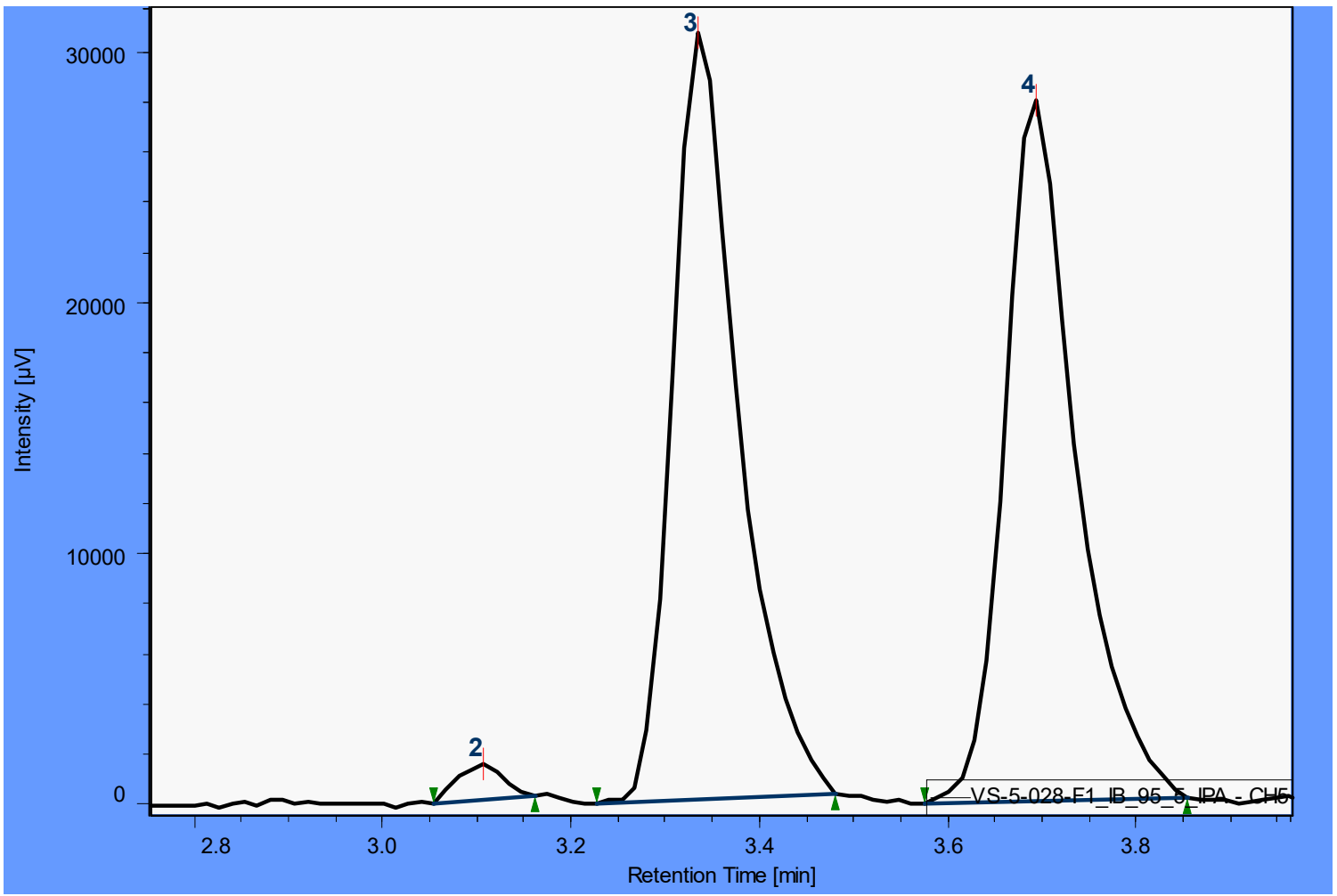

ii. $\mathrm{SFC}$ analysis of $\mathrm{Mb}(\mathrm{H} 64 \mathrm{G}, \mathrm{V} 68 \mathrm{~A})$-catalyzed reaction:

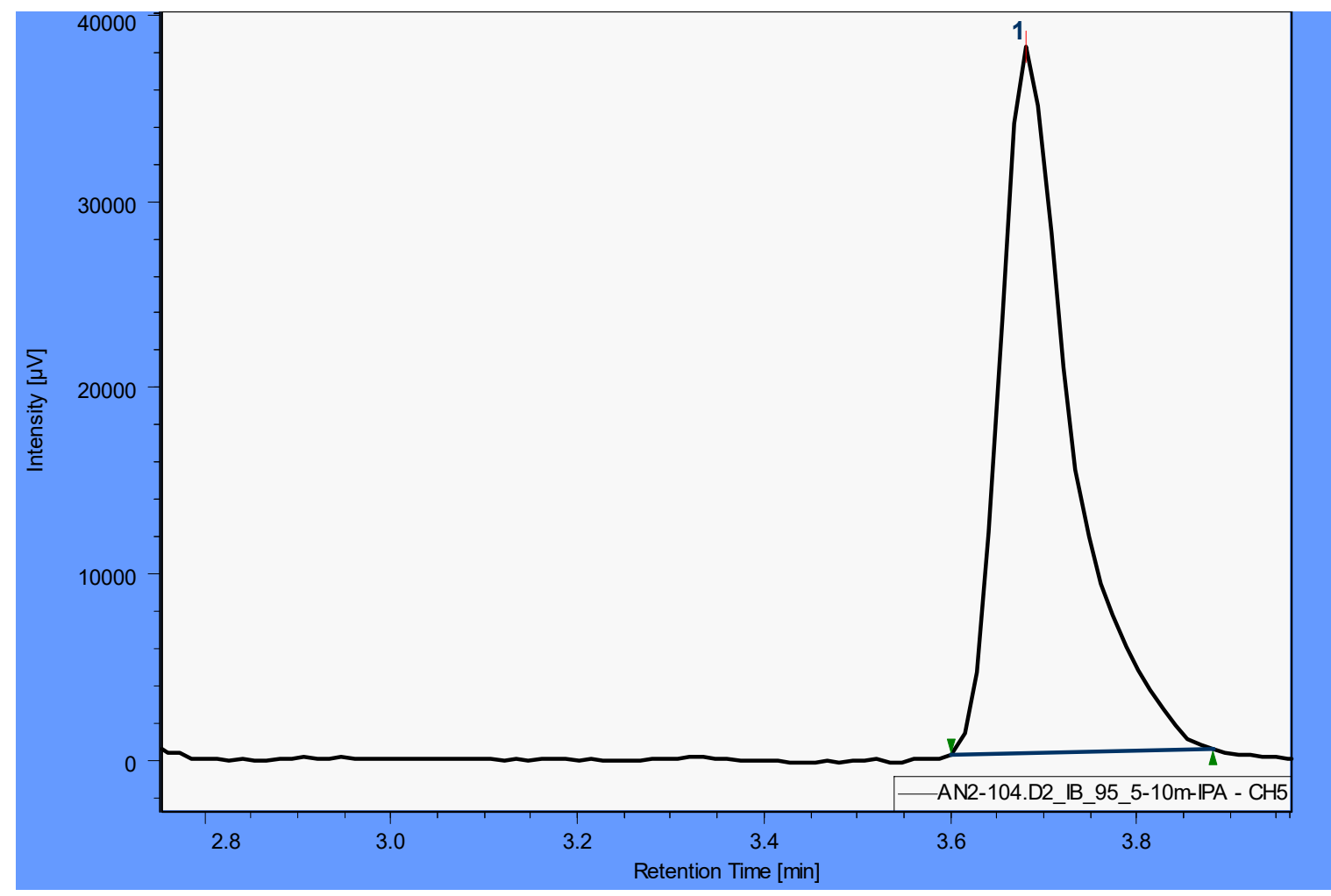


(1) Reaction with 2-isopropenylpyridine $\mathbf{4 k}$ and diazoketone $\mathbf{2}$ to give cyclopropane $\mathbf{5 k}$ :

i. SFC analysis of racemic product $4 \mathbf{k}$ ( 2 cis enantiomers +2 trans enantiomers):

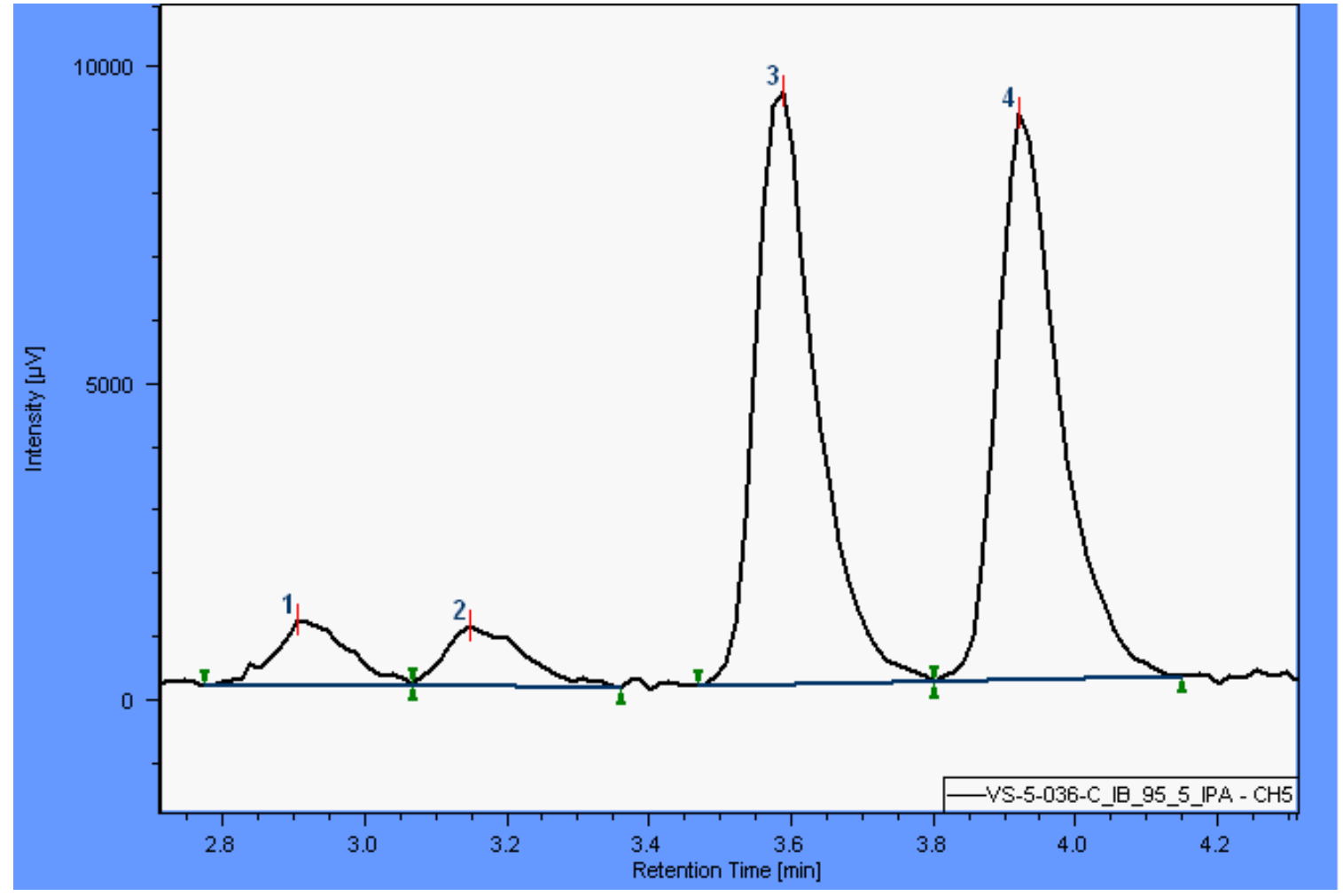

ii. $\mathrm{SFC}$ analysis of $\mathrm{Mb}(\mathrm{H} 64 \mathrm{G}, \mathrm{V} 68 \mathrm{~A})$-catalyzed reaction:

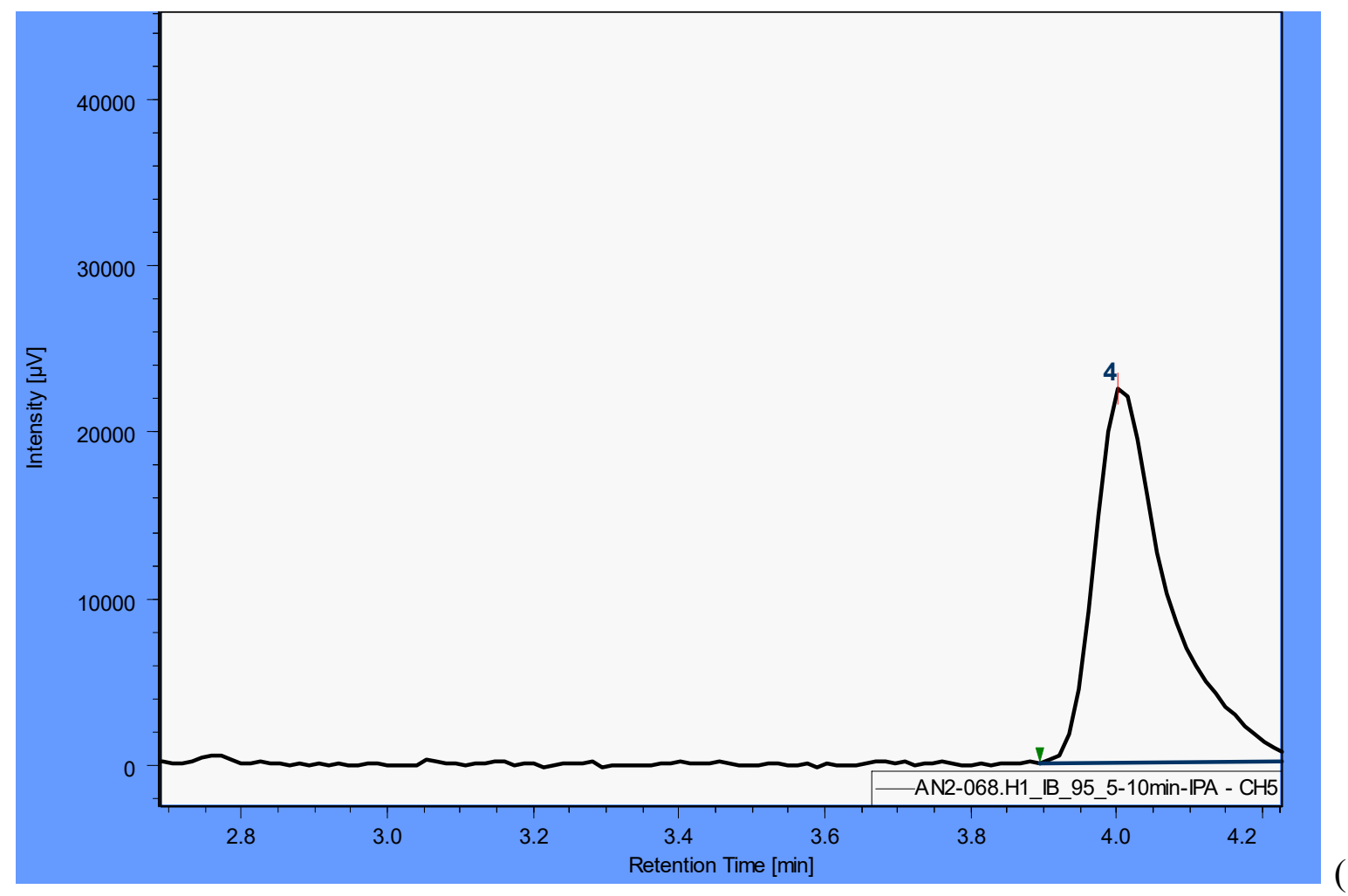


(m) Reaction with styrene $\mathbf{1}$ and diazoketone $\mathbf{6 a}$ to give cyclopropane 7a:

i. SFC analysis of racemic product $7 \mathbf{a}$ ( 2 cis enantiomers +2 trans enantiomers):

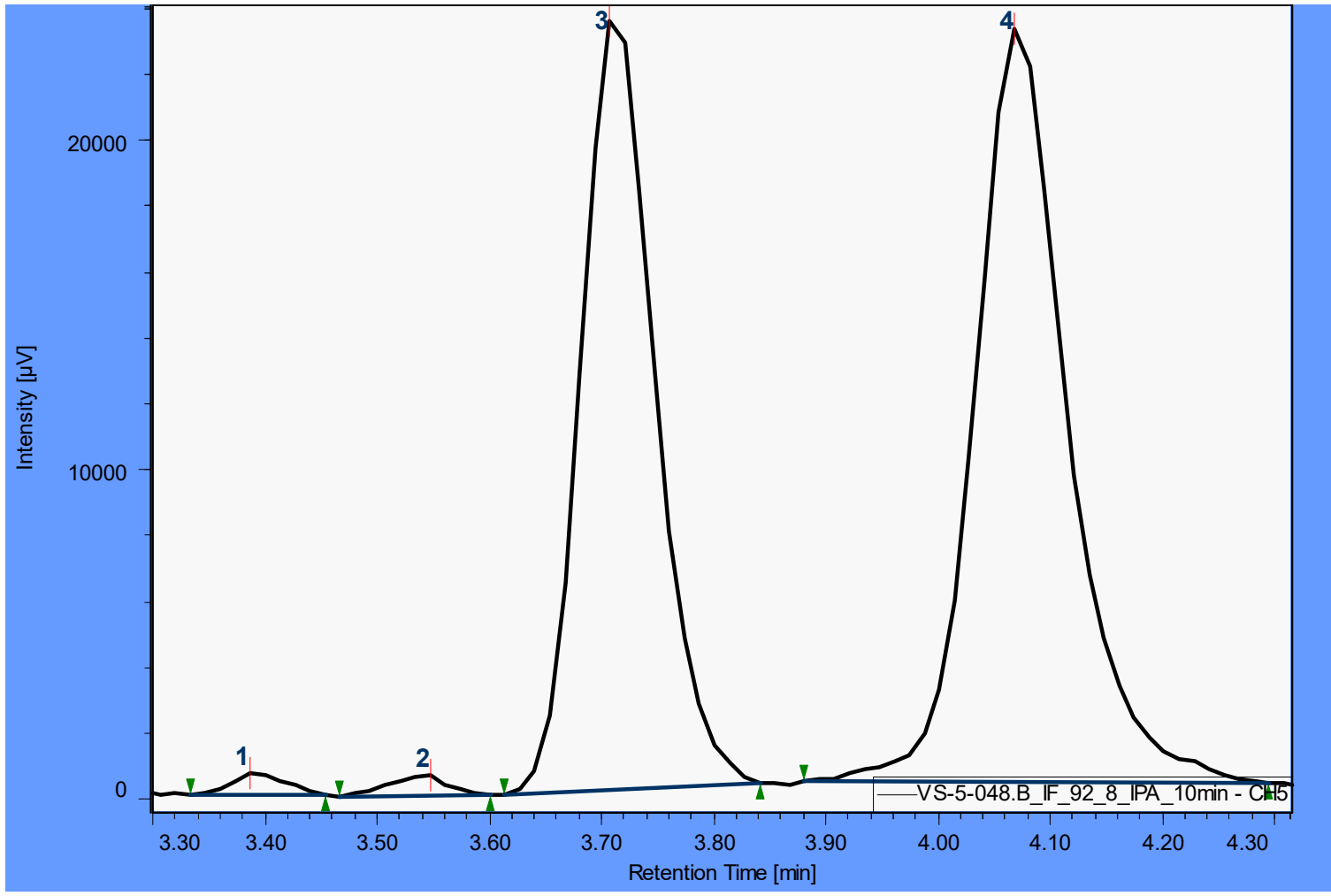

ii. SFC analysis of $\mathrm{Mb}(\mathrm{H} 64 \mathrm{G}, \mathrm{V} 68 \mathrm{~A})$-catalyzed reaction:

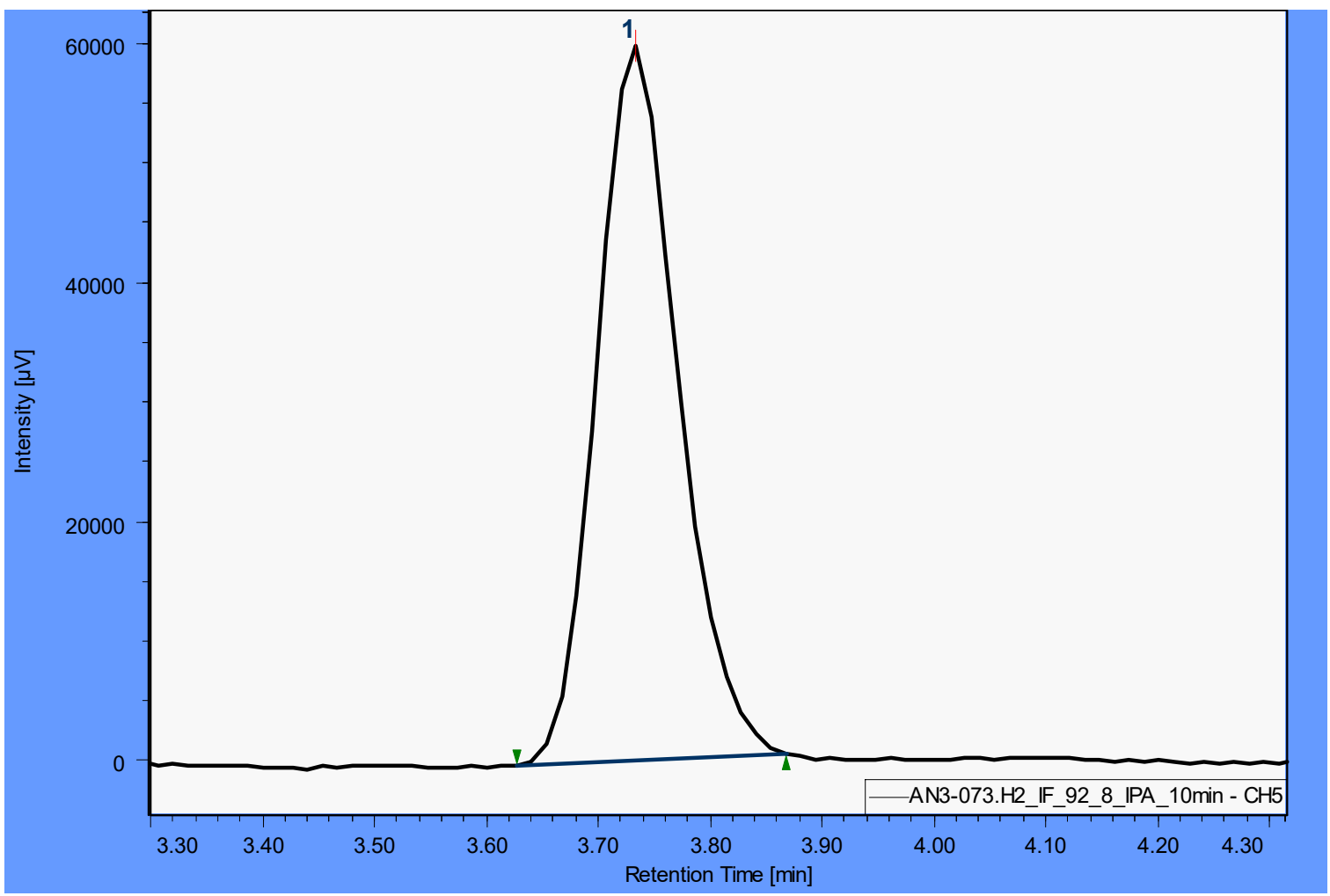


(n) Reaction with styrene $\mathbf{1}$ and diazoketone $\mathbf{6 b}$ to give cyclopropane $\mathbf{7 b}$ :

i. SFC analysis of racemic product $7 \mathbf{b}$ ( 2 trans enantiomers +2 cis enantiomers):

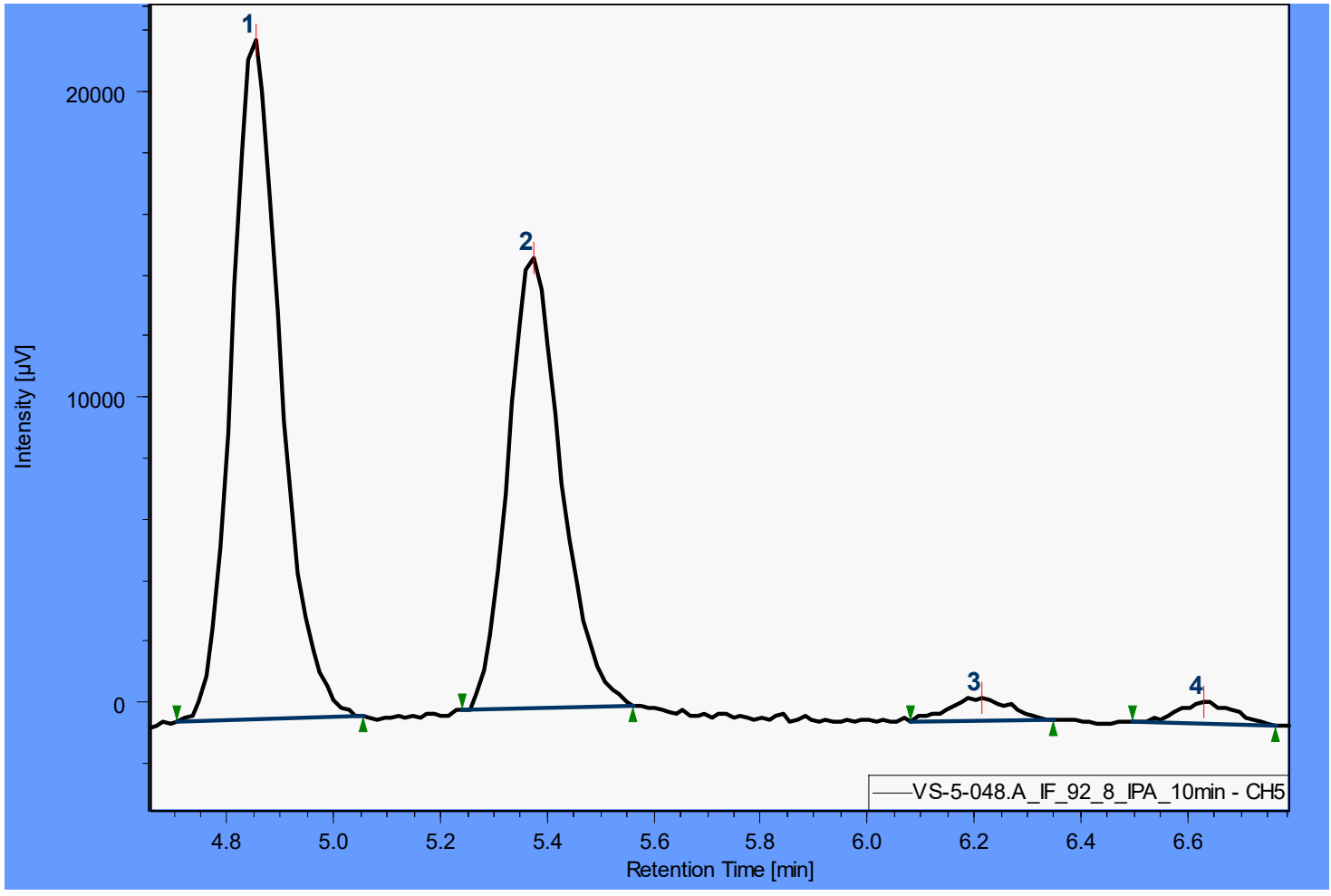

ii. SFC analysis of $\mathrm{Mb}(\mathrm{H} 64 \mathrm{G}, \mathrm{V} 68 \mathrm{~A})$-catalyzed reaction:

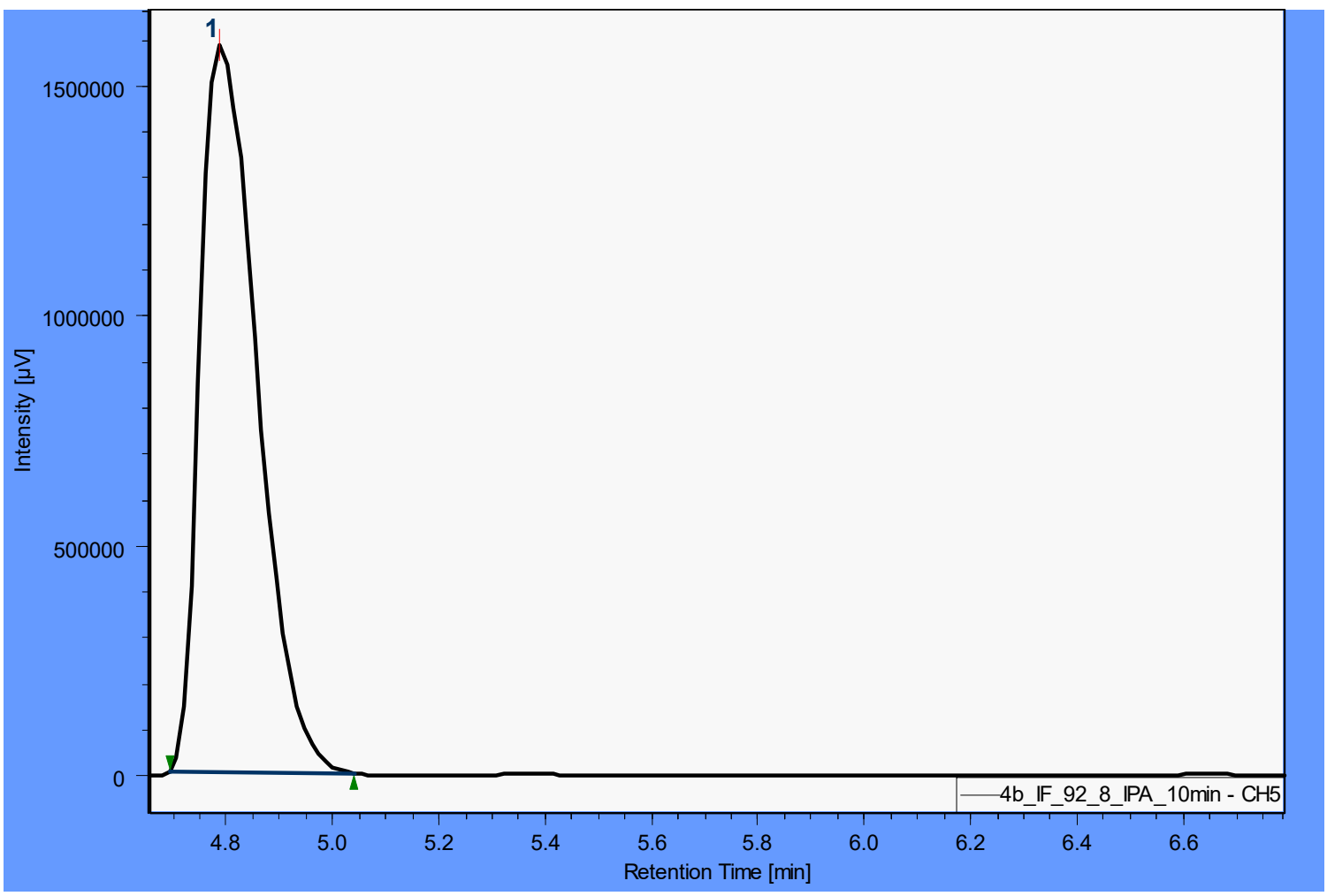


(o) Reaction with styrene $\mathbf{1}$ and diazoketone $\mathbf{6 c}$ to give cyclopropane $\mathbf{7 c}$ :

i. SFC analysis of racemic product $7 \mathbf{c}$ ( 2 trans enantiomers +2 cis enantiomers):

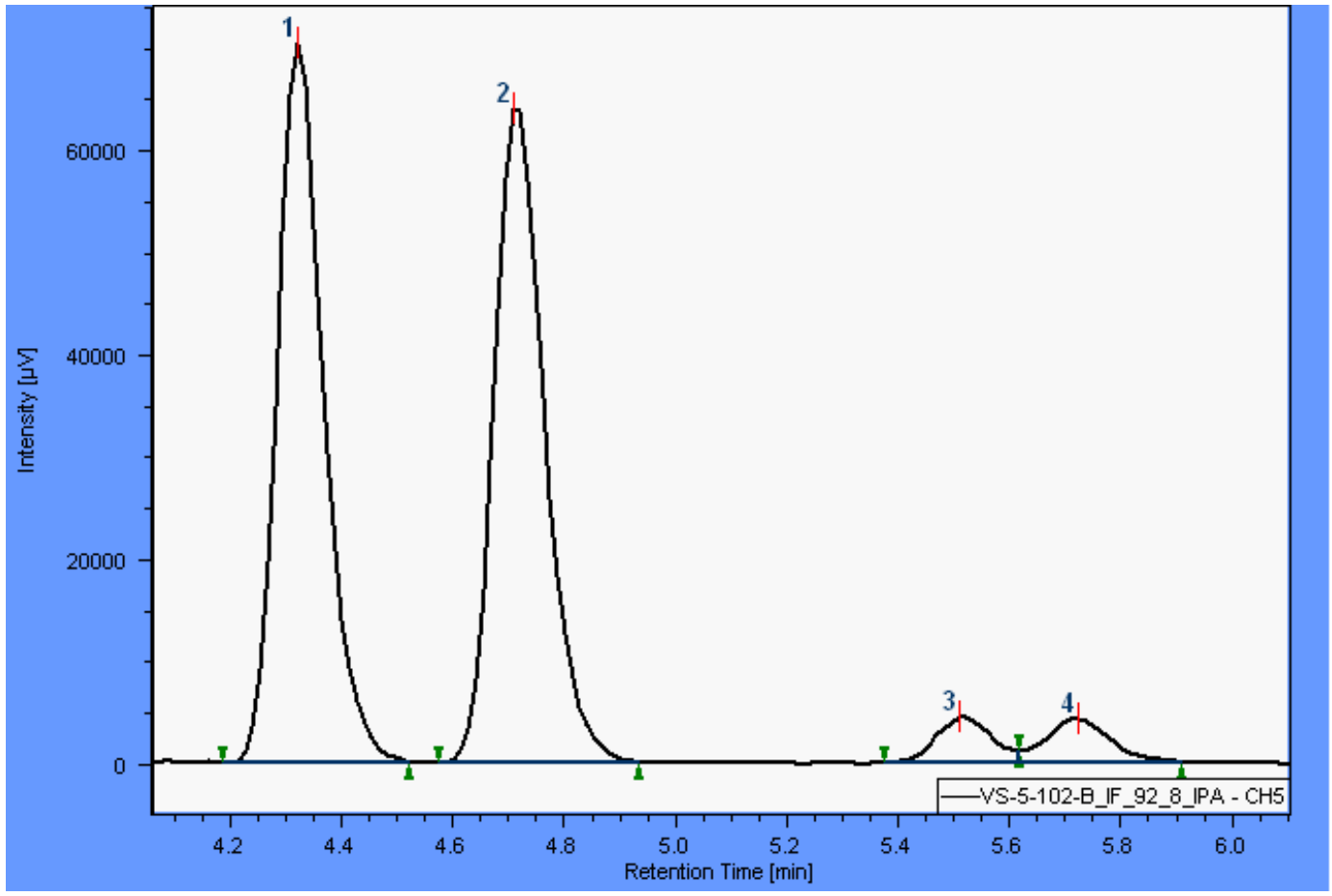

ii. $\mathrm{SFC}$ analysis of $\mathrm{Mb}(\mathrm{H} 64 \mathrm{G}, \mathrm{V} 68 \mathrm{~A})$-catalyzed reaction:

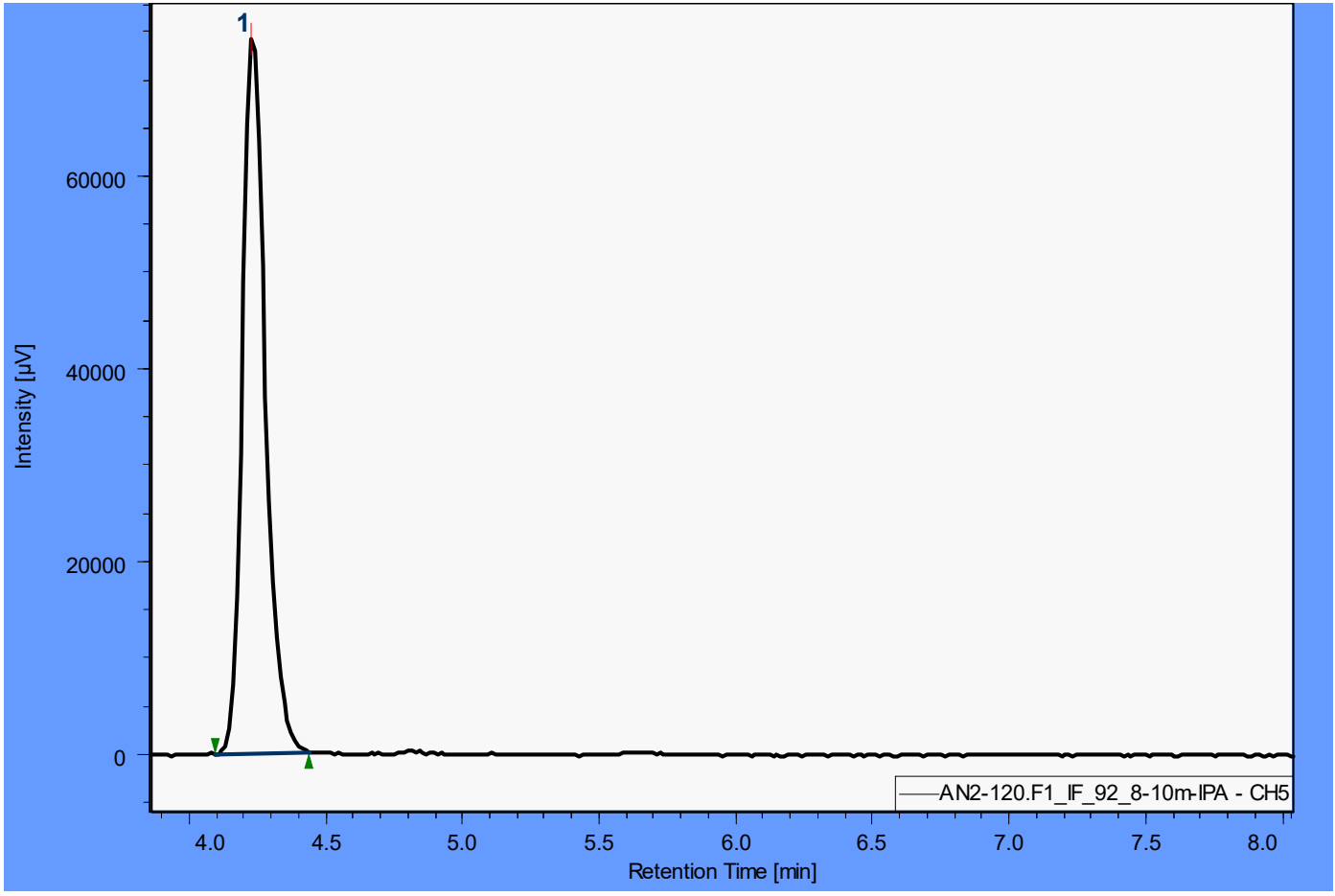


(p) Reaction with styrene $\mathbf{1}$ and diazoketone $\mathbf{6 d}$ to give cyclopropane $\mathbf{7 d}$ :

i. SFC analysis of racemic product $7 \mathbf{d}$ ( 2 trans enantiomers +2 cis enantiomers):

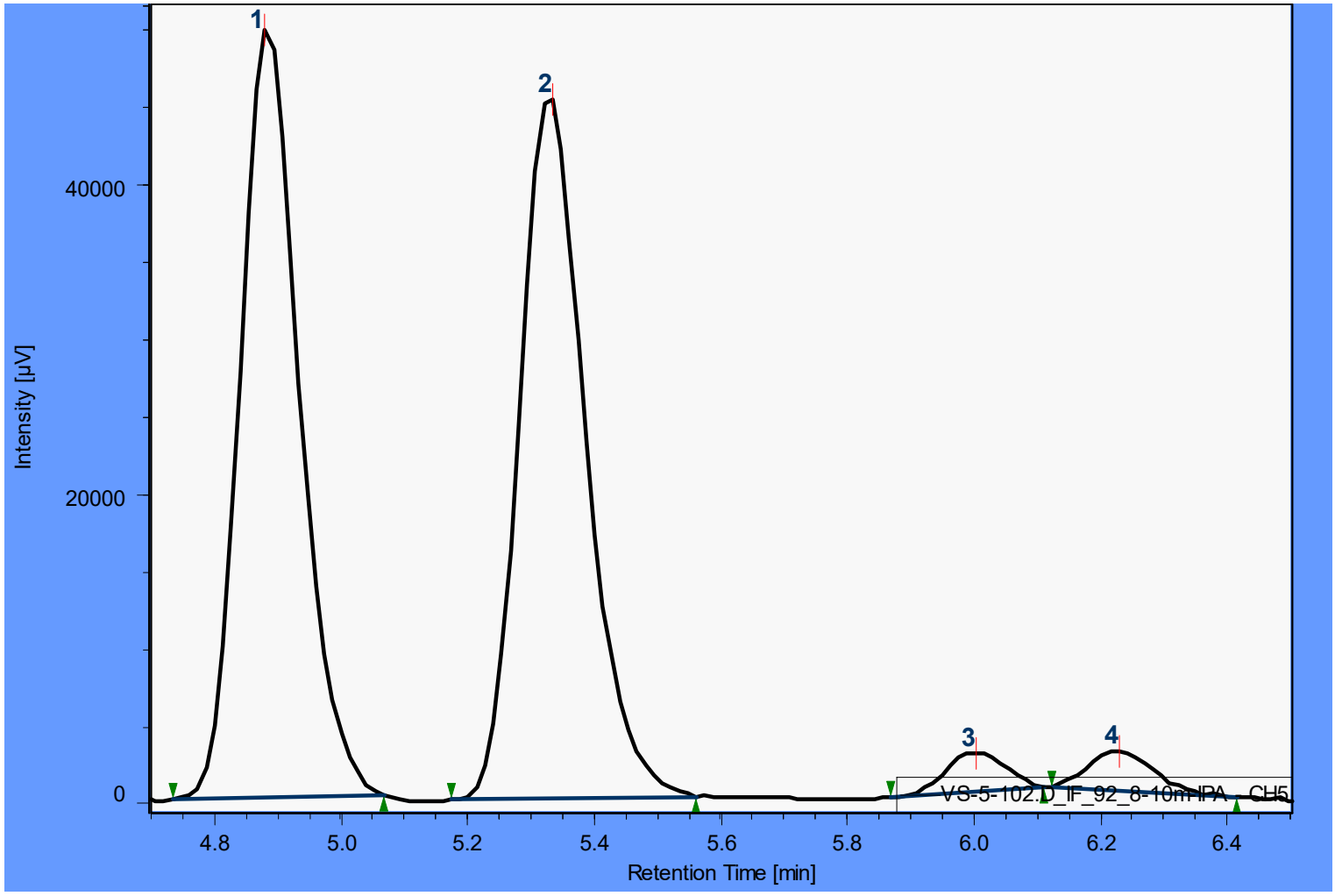

ii. SFC analysis of $\mathrm{Mb}(\mathrm{H} 64 \mathrm{G}, \mathrm{V} 68 \mathrm{~A})$-catalyzed reaction:

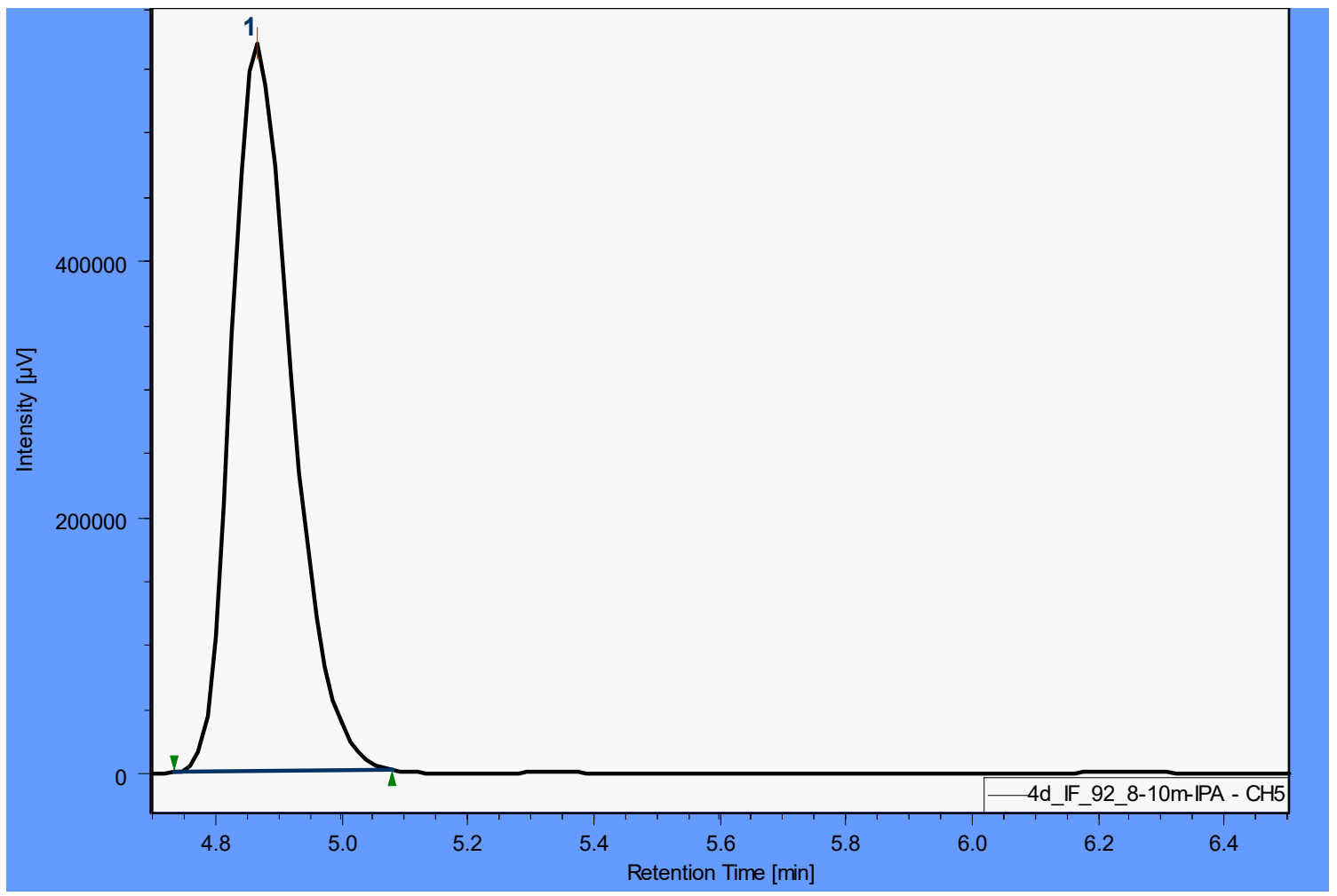


(q) Reaction with styrene $\mathbf{1}$ and diazoketone $\mathbf{6 e}$ to give cyclopropane 7e:

i. SFC analysis of racemic product $7 \mathbf{e}$ ( 2 trans enantiomers +2 cis enantiomers):

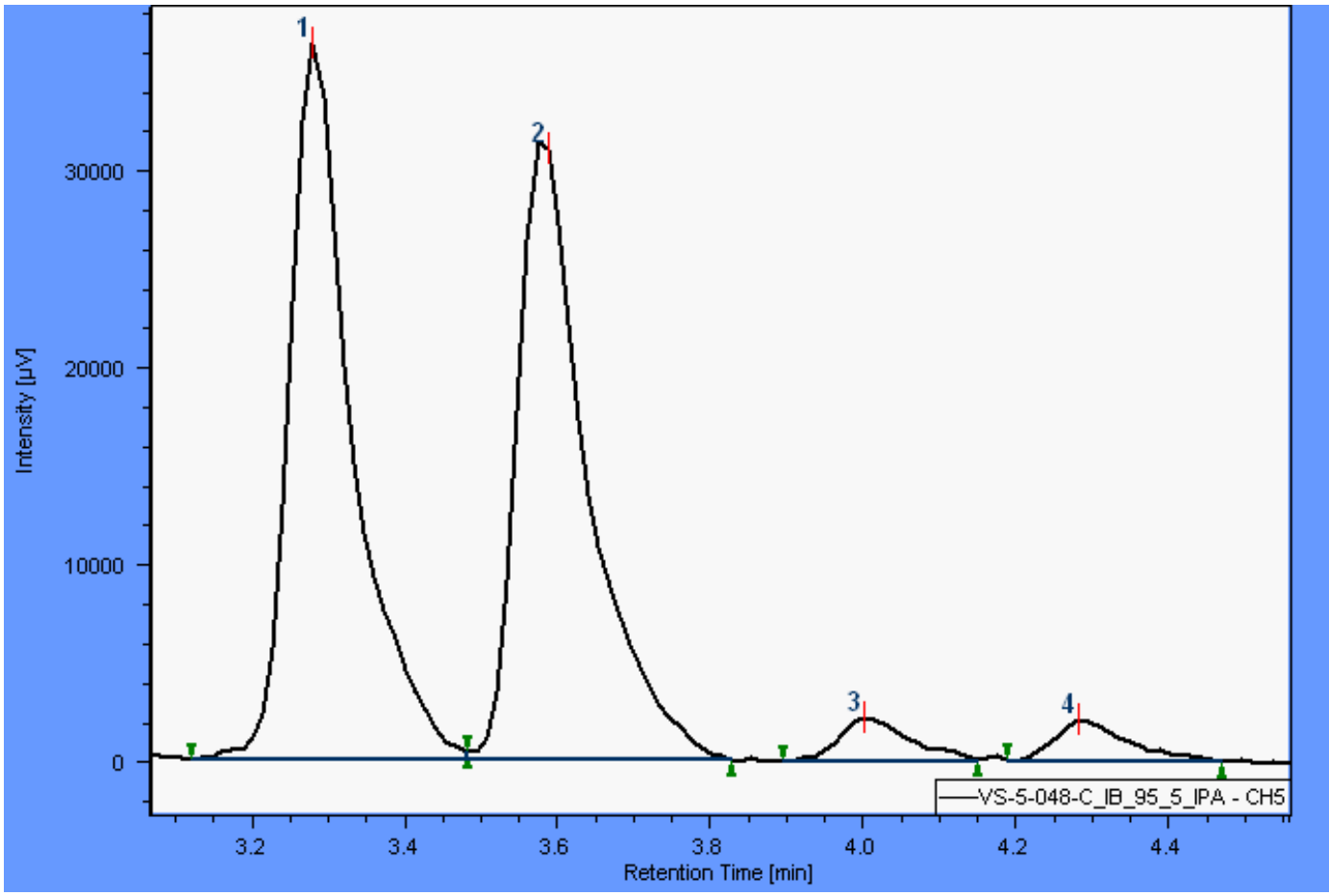

ii. $\mathrm{SFC}$ analysis of $\mathrm{Mb}(\mathrm{H} 64 \mathrm{G}, \mathrm{V} 68 \mathrm{~A})$-catalyzed reaction:

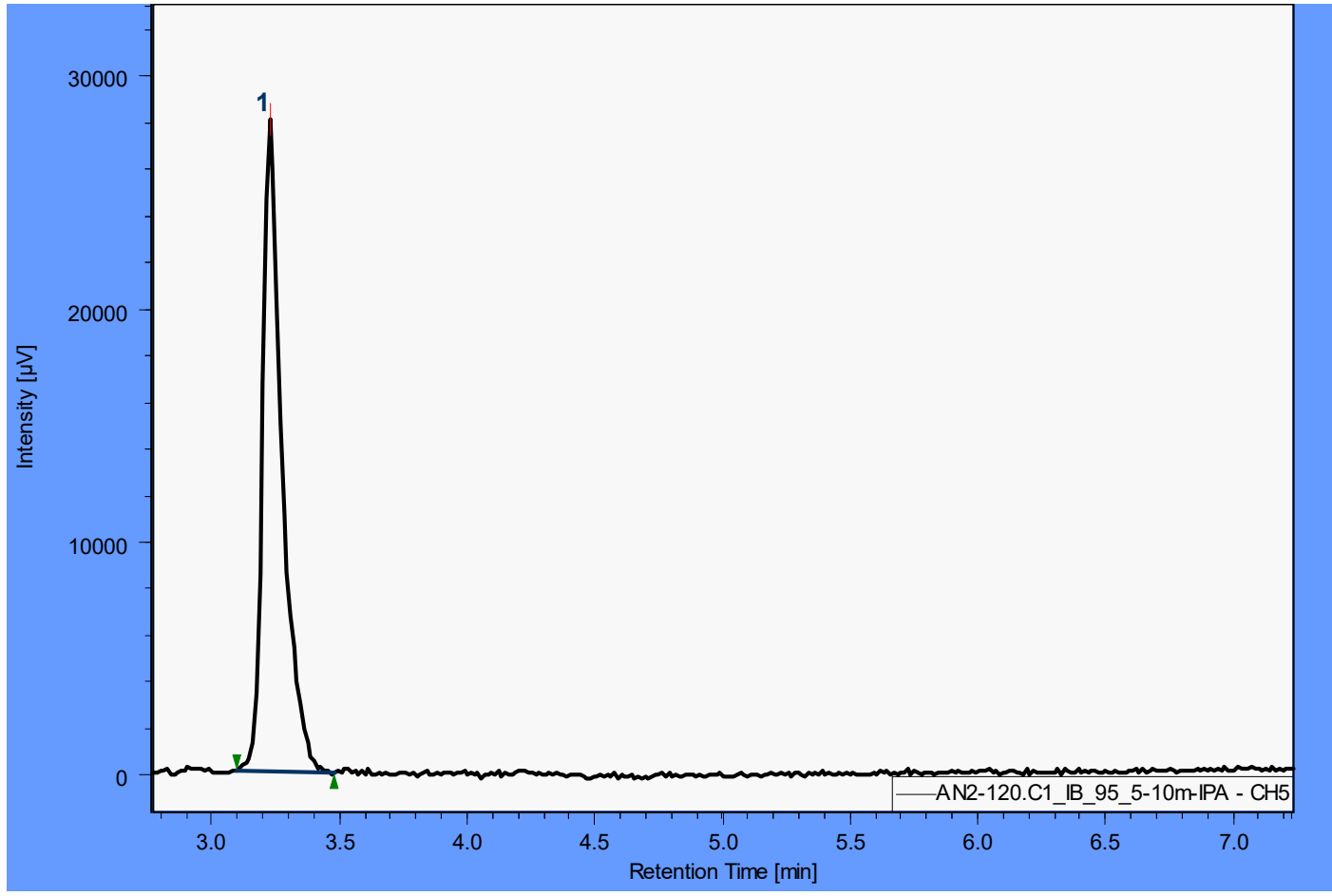


(r) Reaction with styrene $\mathbf{1}$ and diazoketone $\mathbf{6 f}$ to give cyclopropane $\mathbf{7 f}$ :

i. SFC analysis of racemic product $7 \mathbf{f}$ ( 2 trans enantiomers + unresolved enantiomers):

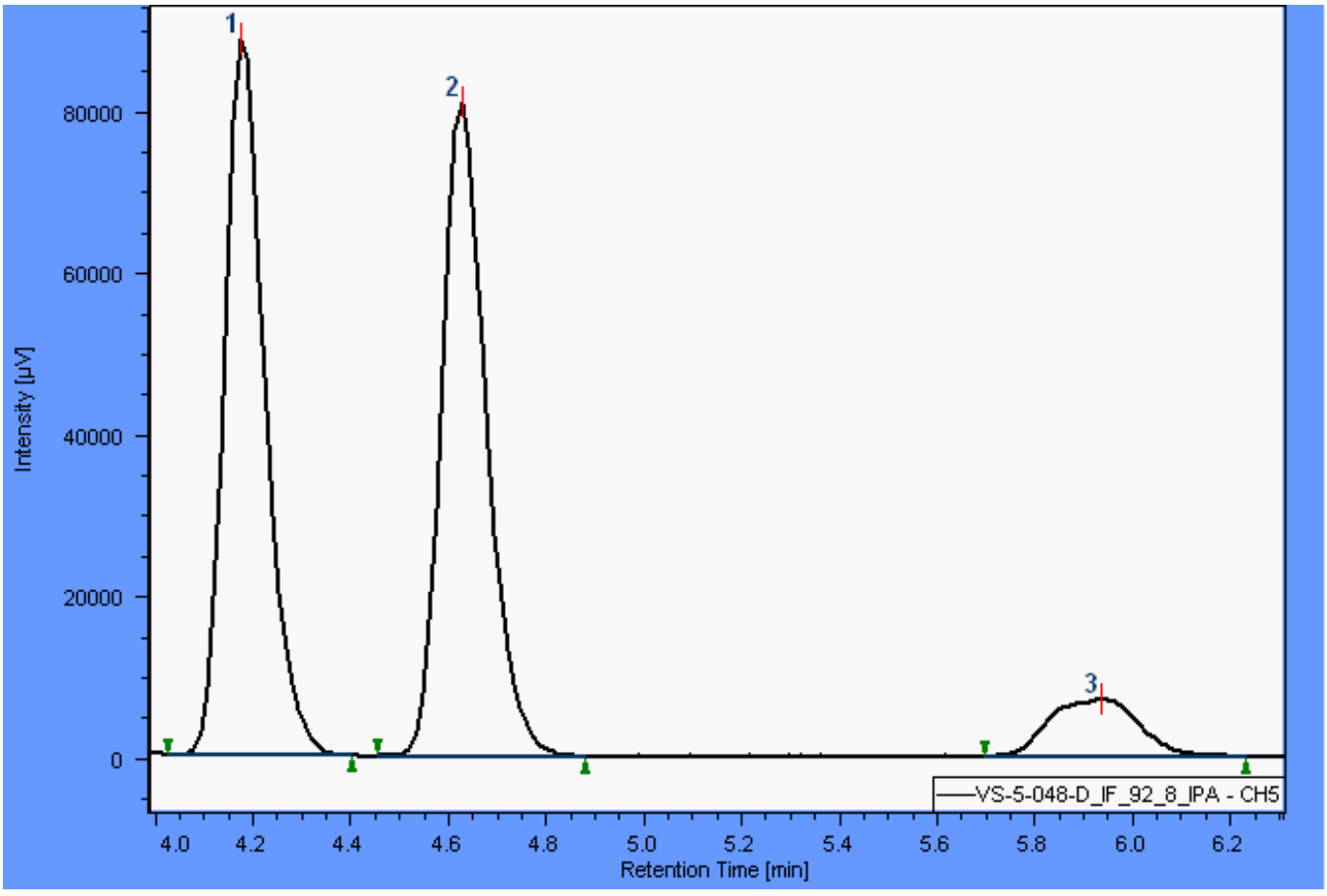

ii. $\mathrm{SFC}$ analysis of $\mathrm{Mb}(\mathrm{H} 64 \mathrm{G}, \mathrm{V} 68 \mathrm{~A})$-catalyzed reaction:

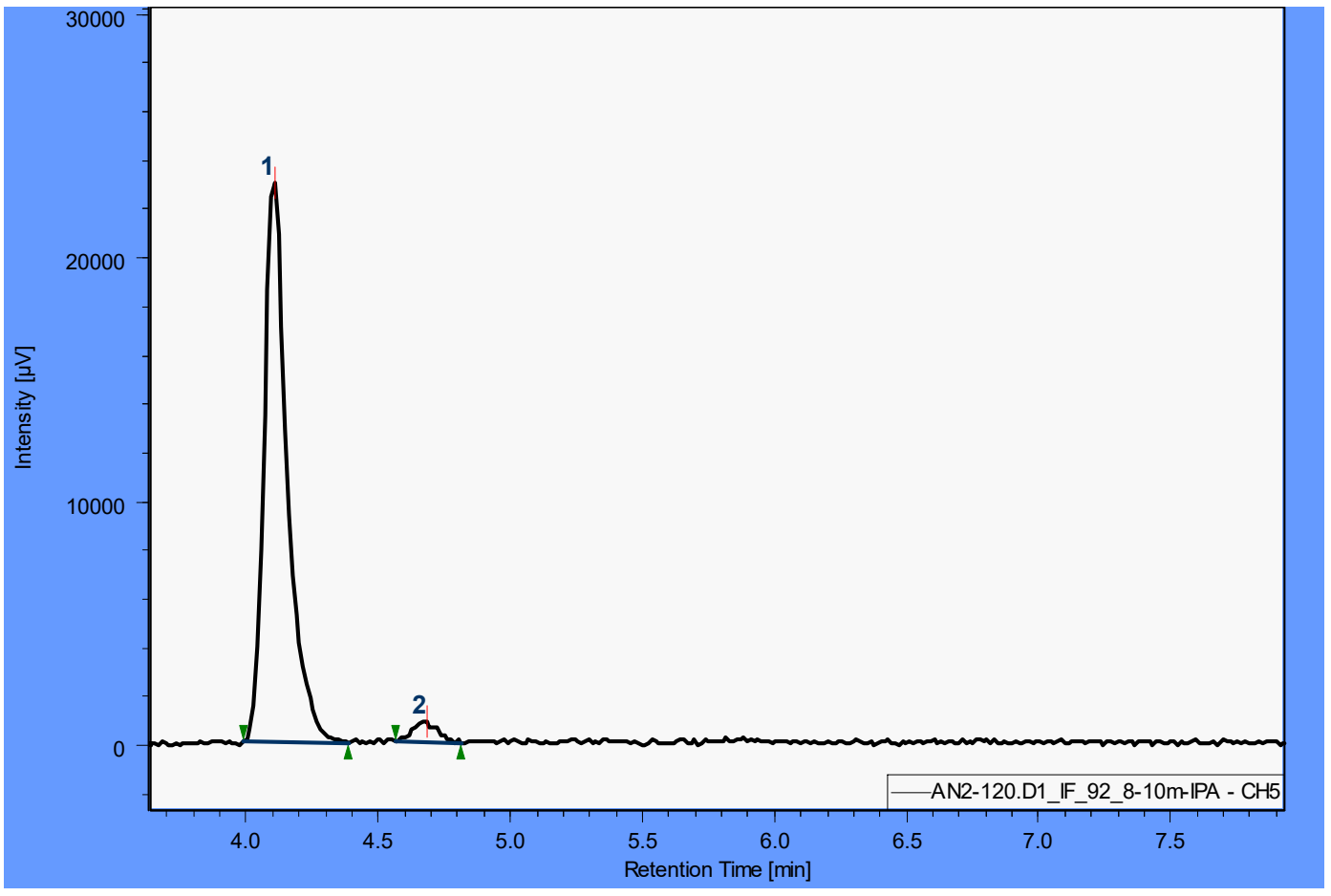


(s) Reaction with styrene $\mathbf{1}$ and diazoketone $\mathbf{6 g}$ to give cyclopropane $\mathbf{7 g}$ :

i. SFC analysis of racemic product $7 \mathbf{g}$ ( 2 trans enantiomers +2 cis enantiomers):

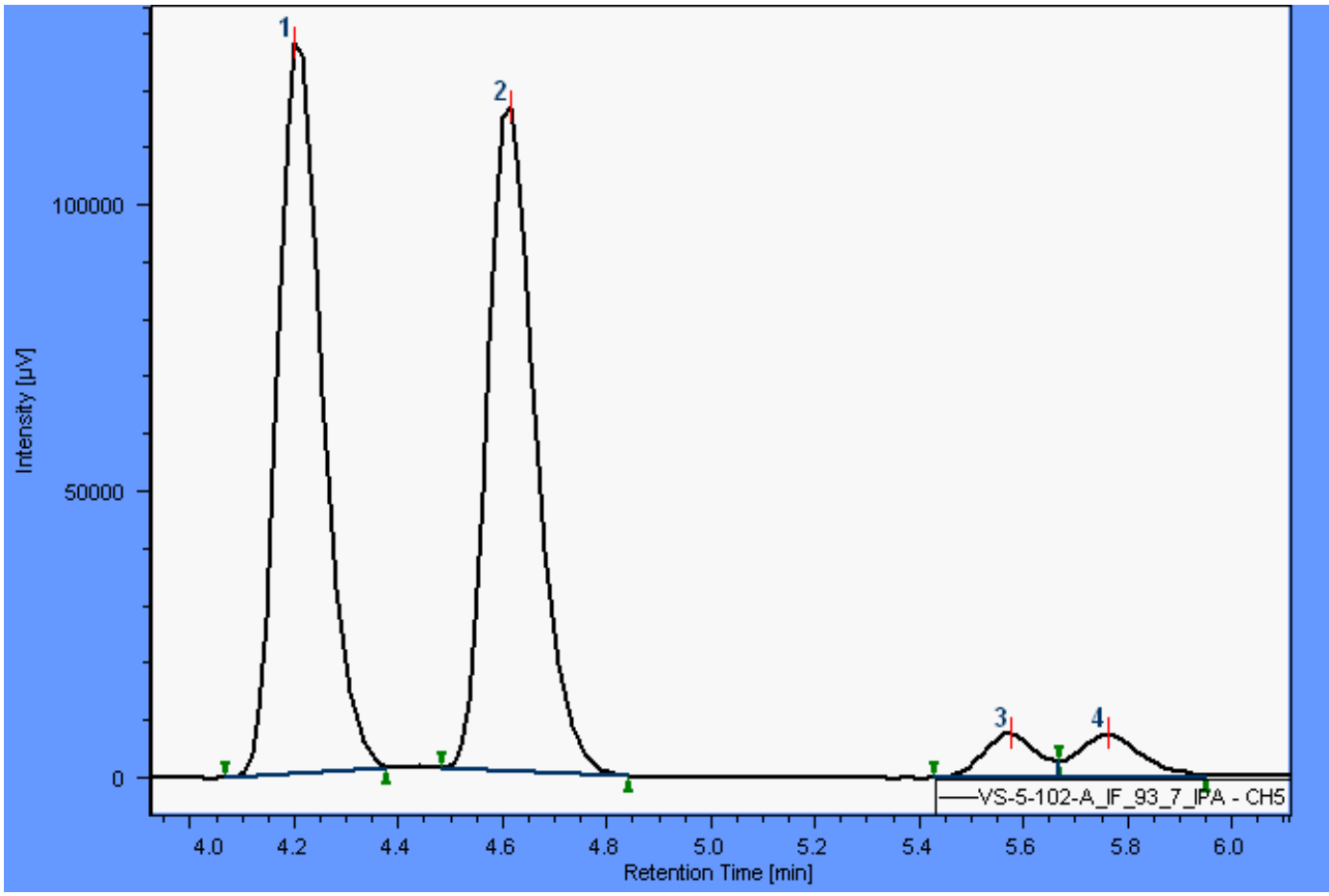

ii. SFC analysis of $\mathrm{Mb}(\mathrm{H} 64 \mathrm{G}, \mathrm{V} 68 \mathrm{~A})$-catalyzed reaction:

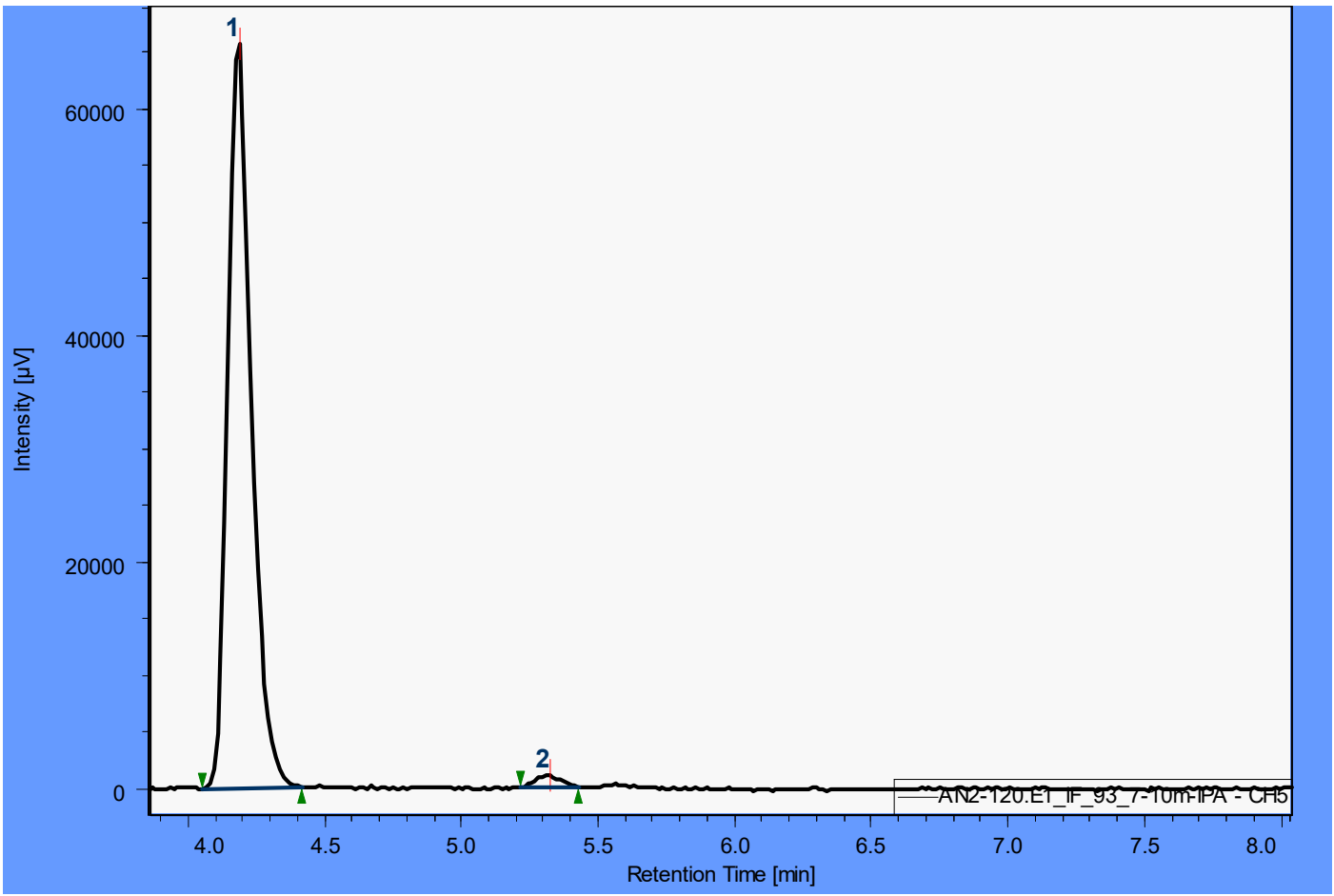


(t) Reaction with styrene $\mathbf{1}$ and diazoketone $\mathbf{6 h}$ to give cyclopropane $\mathbf{7 h}$ :

i. SFC analysis of racemic product $7 \mathbf{h}$ ( 2 trans enantiomers +2 cis enantiomers):

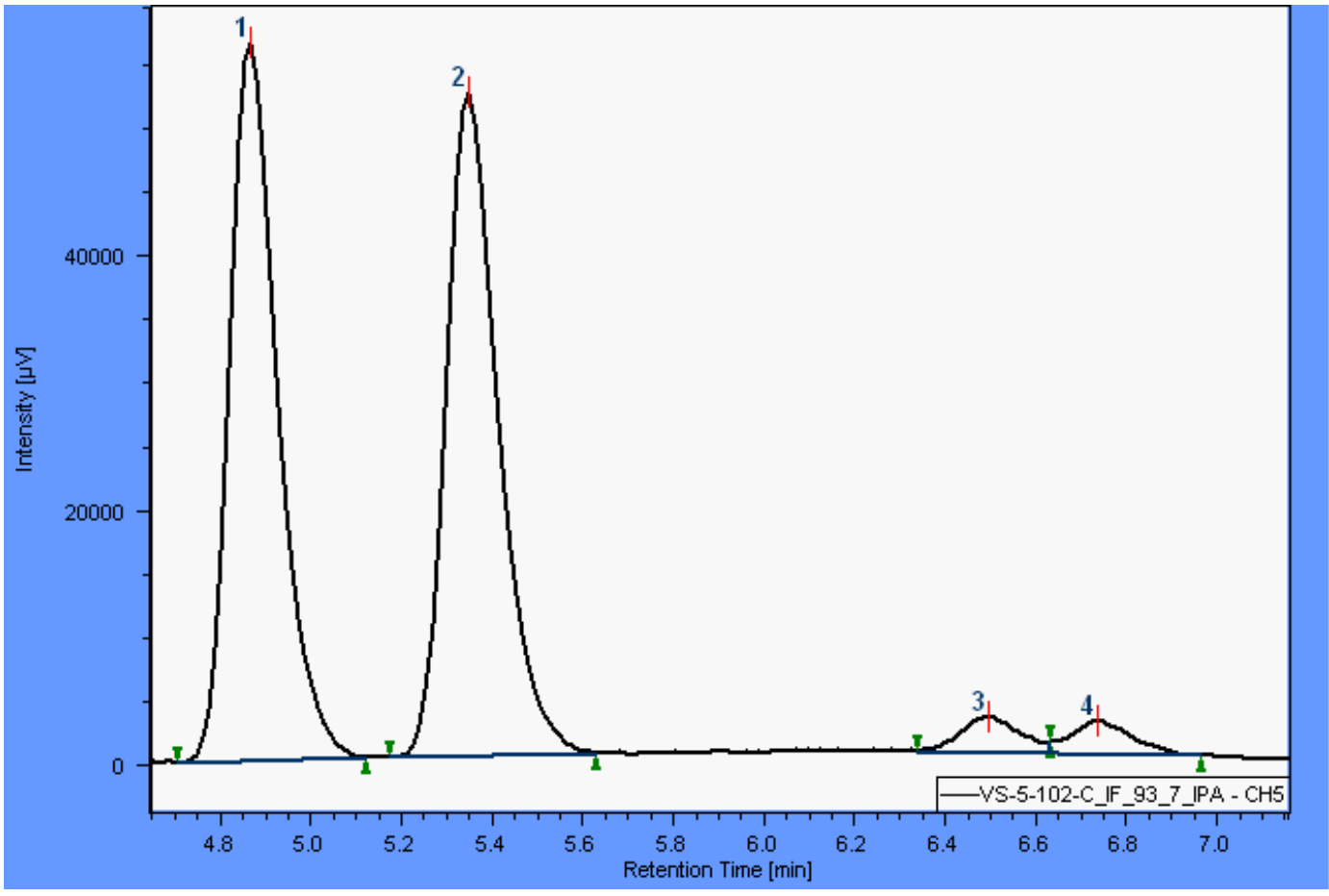

ii. $\mathrm{SFC}$ analysis of $\mathrm{Mb}(\mathrm{H} 64 \mathrm{G}, \mathrm{V} 68 \mathrm{~A})$-catalyzed reaction:

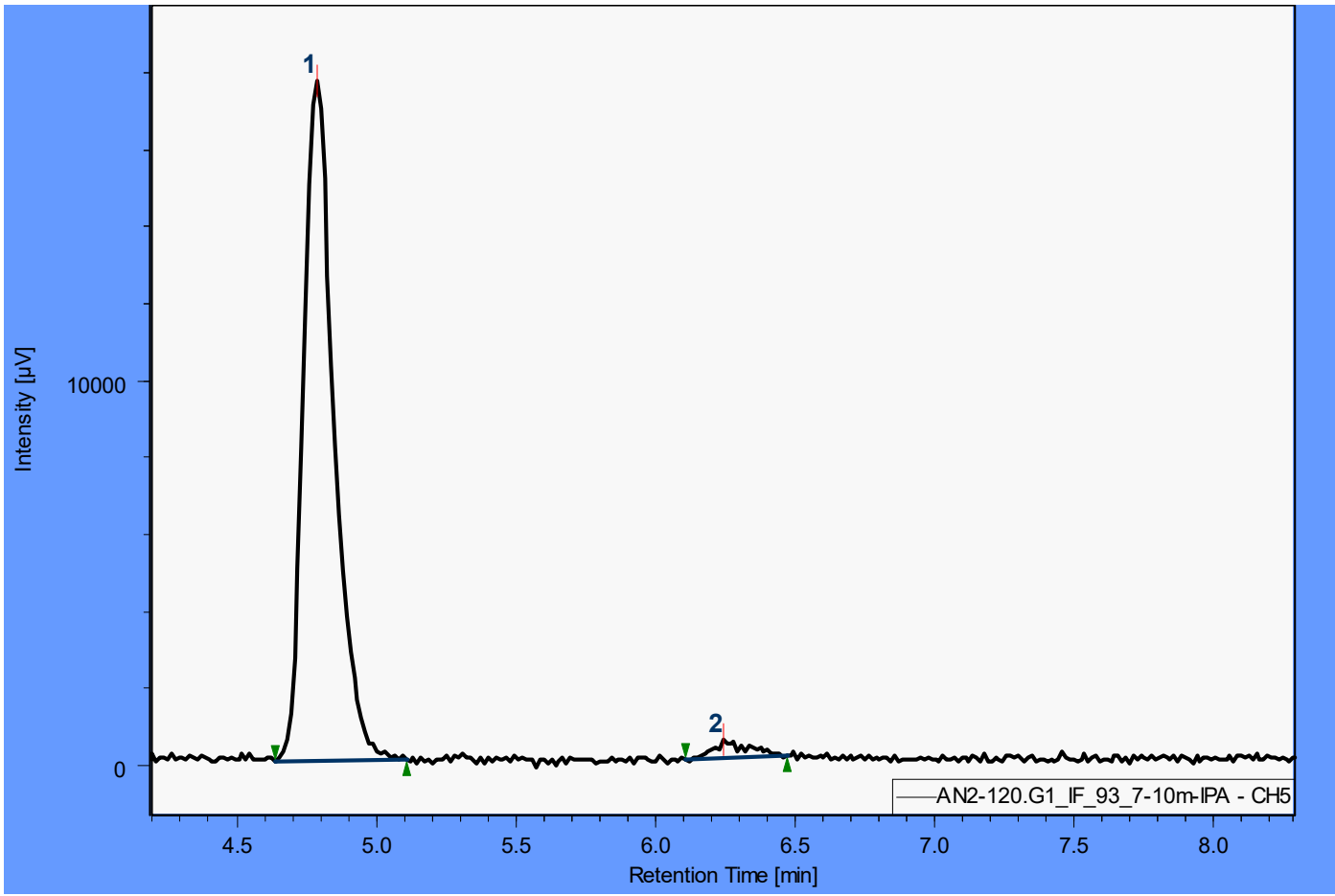


(u) Reaction with styrene $\mathbf{1}$ and diazoketone $\mathbf{6 i}$ to give cyclopropane 7i:

i. SFC analysis of racemic product $7 \mathbf{i}$ ( 2 trans enantiomers + unresolved enantiomers):

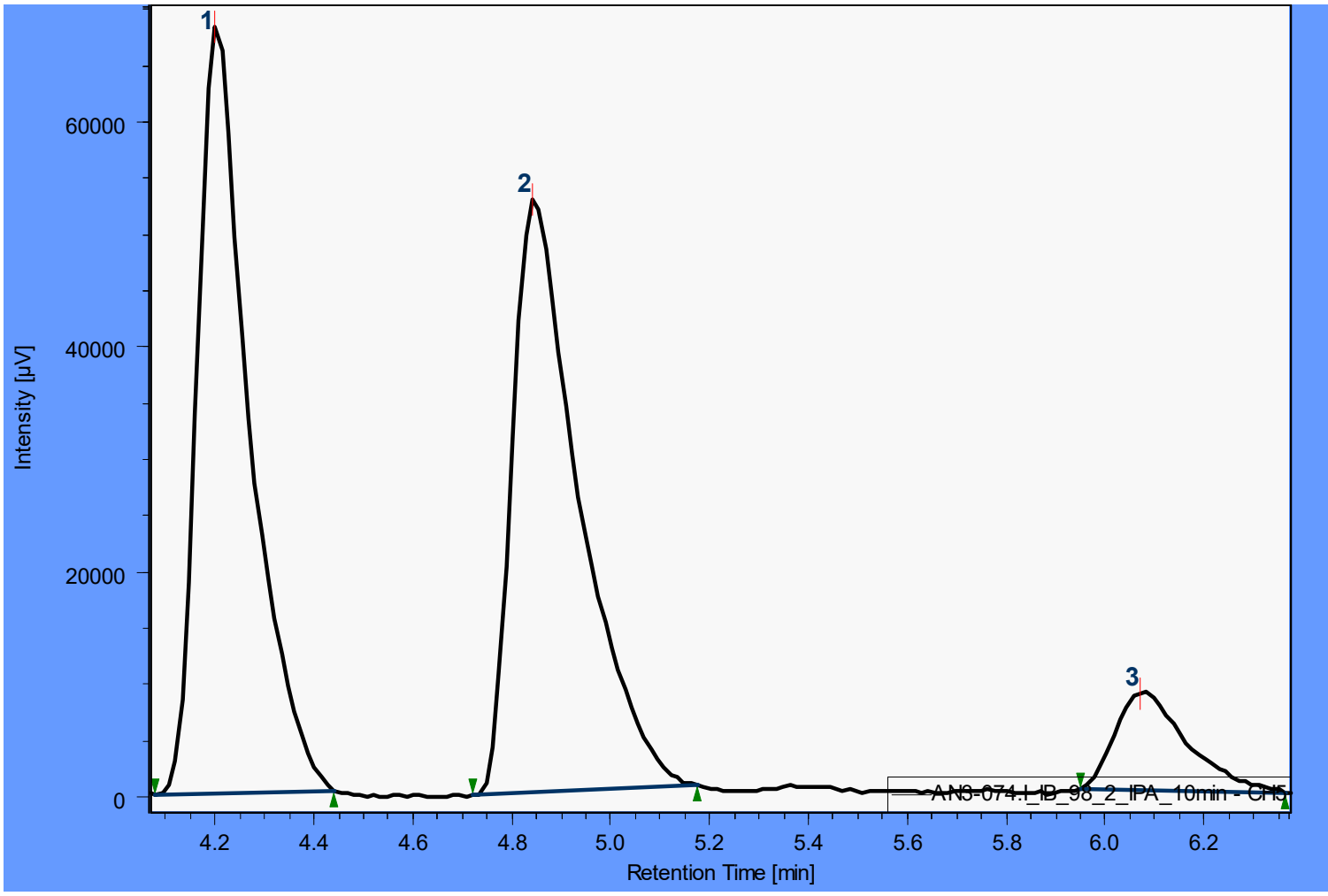

ii. SFC analysis of $\mathrm{Mb}(\mathrm{H} 64 \mathrm{G}, \mathrm{V} 68 \mathrm{~A})$-catalyzed reaction:

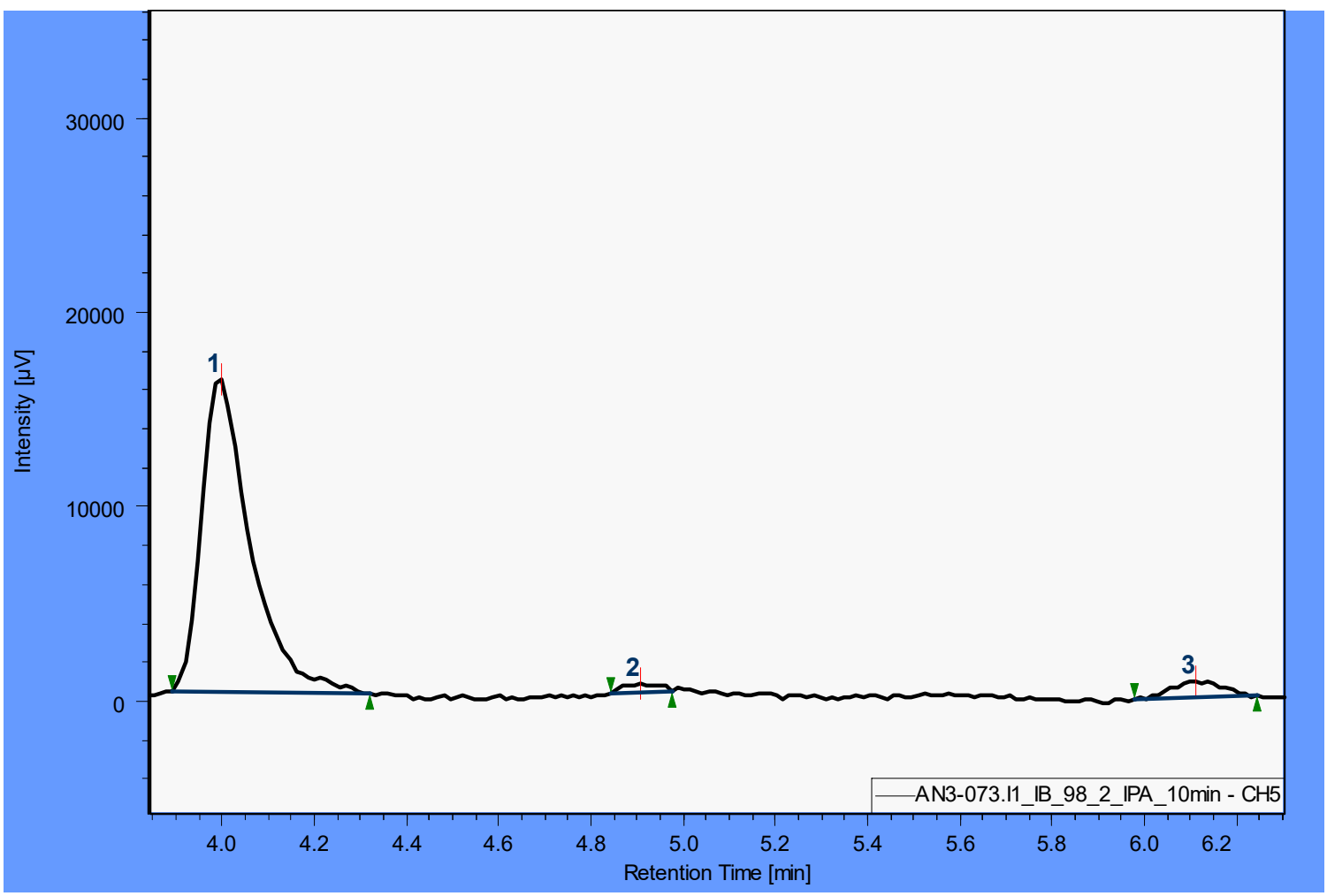


(v) Reaction with styrene $\mathbf{1}$ and diazoketone $\mathbf{6 k}$ to give cyclopropane $\mathbf{7 k}$ :

i. Chiral GC analysis of racemic standard (2 cis enantiomers + unresolved trans enantiomers):

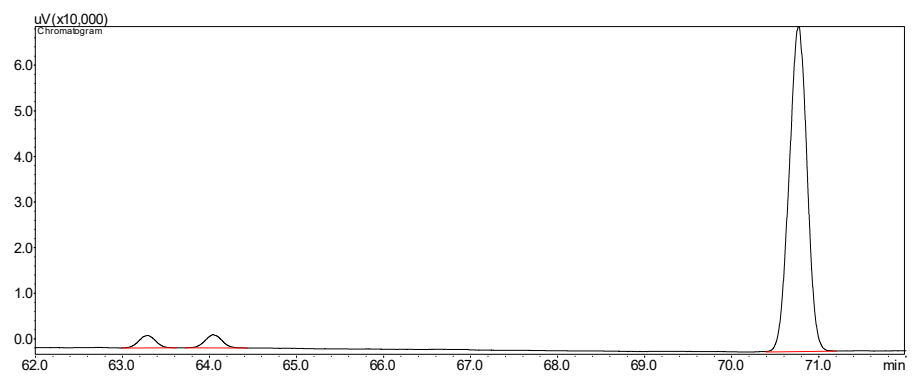

ii. Chiral GC analysis of $\mathbf{7 k}$ from $\mathrm{Mb}(\mathrm{H} 64 \mathrm{G}, \mathrm{V} 68 \mathrm{~A})$-catalyzed reaction:

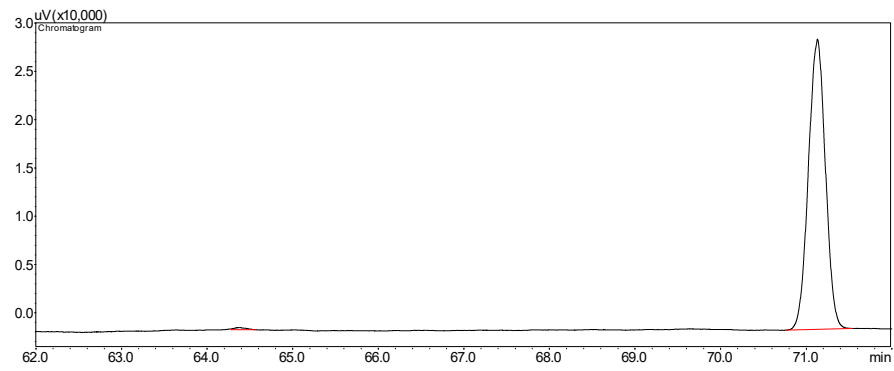

iii. Chiral HPLC analysis of racemic trans-7k (styrene starting material +2 trans enantiomers):

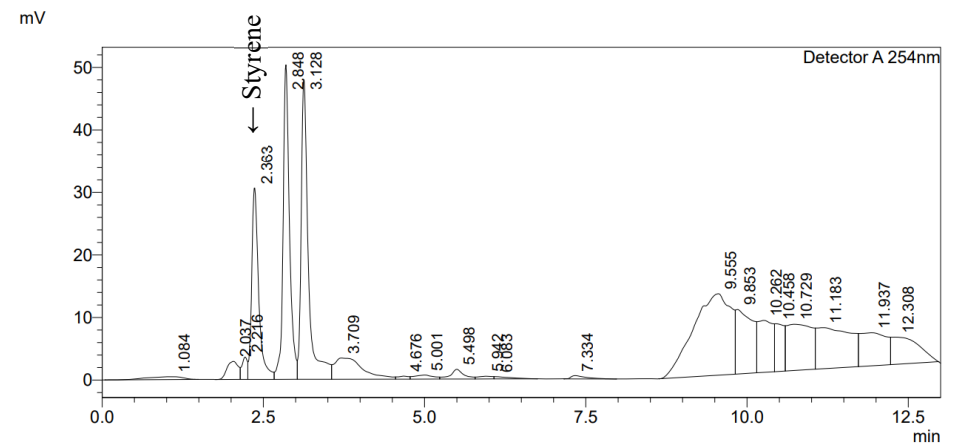

iv. Chiral HPLC analysis of $7 \mathbf{k}$ from Mb(H64G,V68A)-catalyzed reaction:

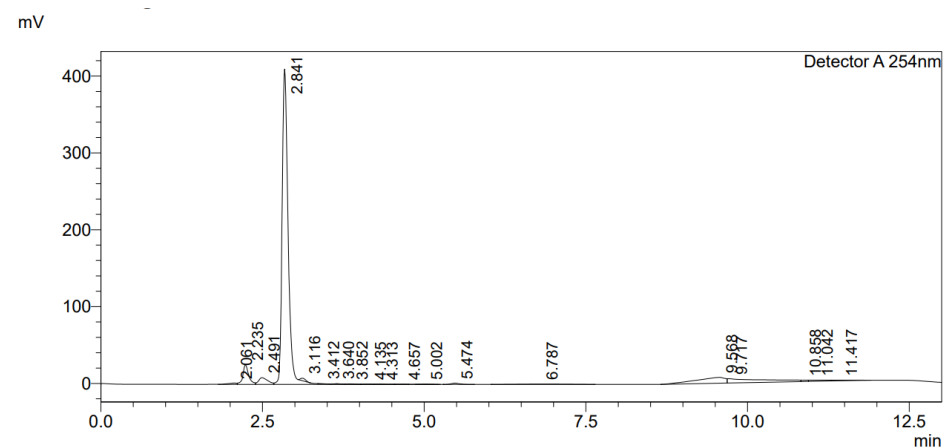


(w) Reaction with styrene $\mathbf{1}$ and diazoketone $\mathbf{6} \mathbf{l}$ to give cyclopropane 7l:

i. Chiral GC analysis of racemic standard $(2$ cis enantiomers + unresolved trans enantiomers):

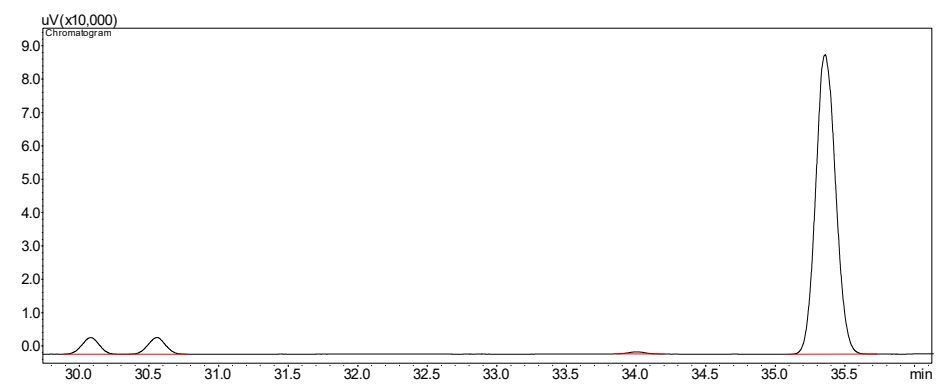

ii. Chiral GC analysis of $\mathbf{7 l}$ from $\mathrm{Mb}(\mathrm{H} 64 \mathrm{G}, \mathrm{V} 68 \mathrm{~A})$-catalyzed reaction:

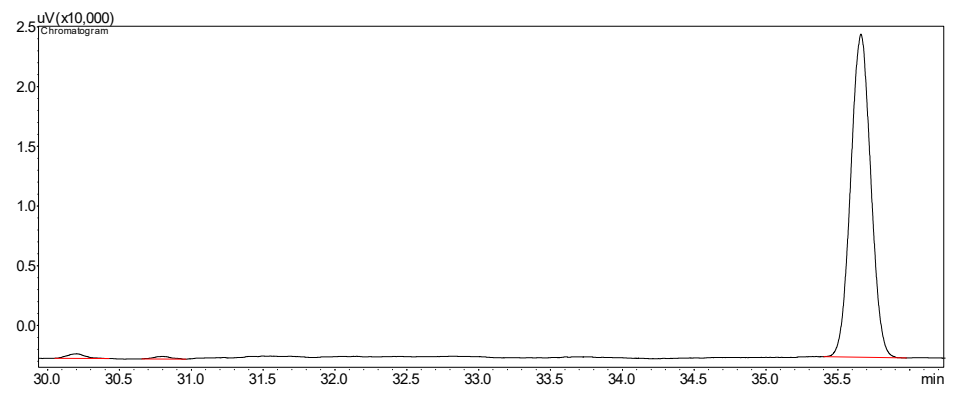

iii. Chiral HPLC analysis of racemic trans-7l (styrene starting material +2 trans enantiomers):

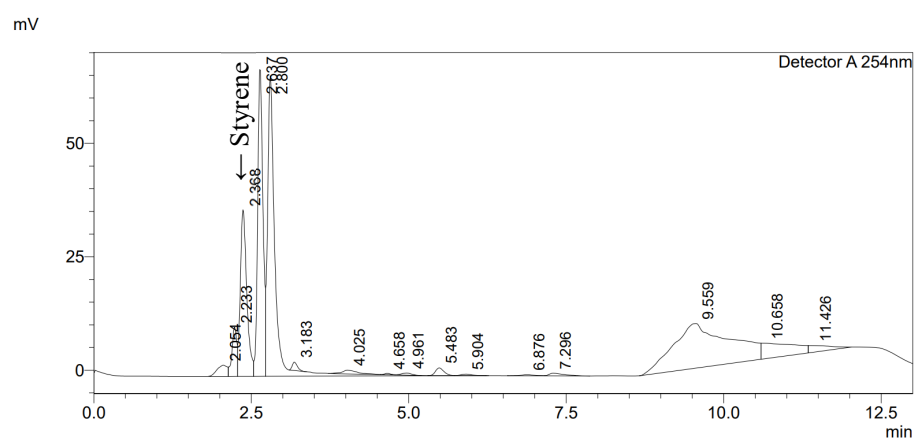

iv. Chiral HPLC analysis of 71 from Mb(H64G,V68A)-catalyzed reaction:

$\mathrm{mV}$

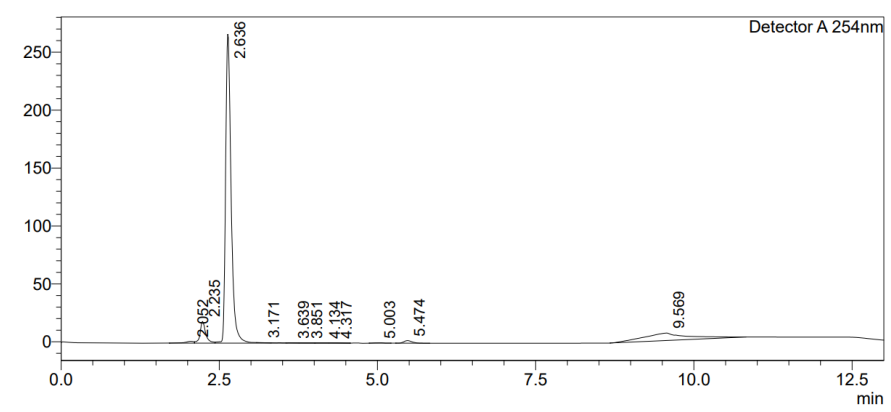


(x) Reaction with styrene $\mathbf{1}$ and diazoketone $\mathbf{6 m}$ to give cyclopropane $\mathbf{7 m}$ :

i. Chiral GC analysis of racemic standard (2 cis enantiomers + partially resolved 2 trans enantiomers):

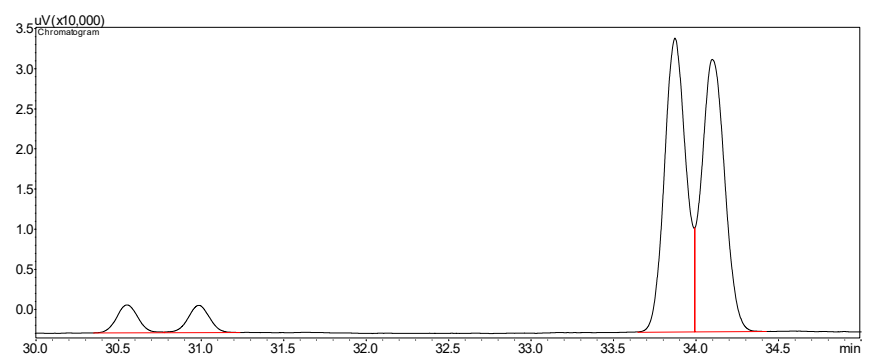

ii. Chiral GC analysis of $\mathbf{7 m}$ from $\mathrm{Mb}(\mathrm{H} 64 \mathrm{G}, \mathrm{V} 68 \mathrm{~A})$-catalyzed reaction:

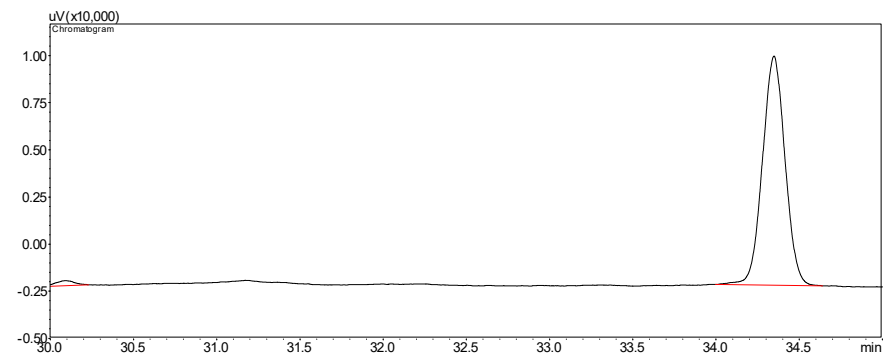

iii. Chiral HPLC analysis of racemic trans $\mathbf{- 7 m}$ (styrene starting material +2 trans enantiomers):

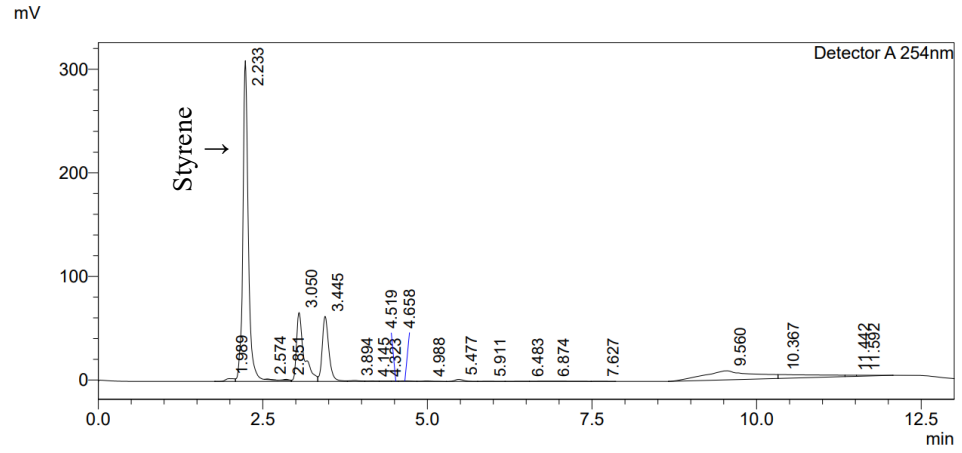

iv. Chiral HPLC analysis of $\mathbf{7 m}$ from Mb(H64G,V68A)-catalyzed reaction:

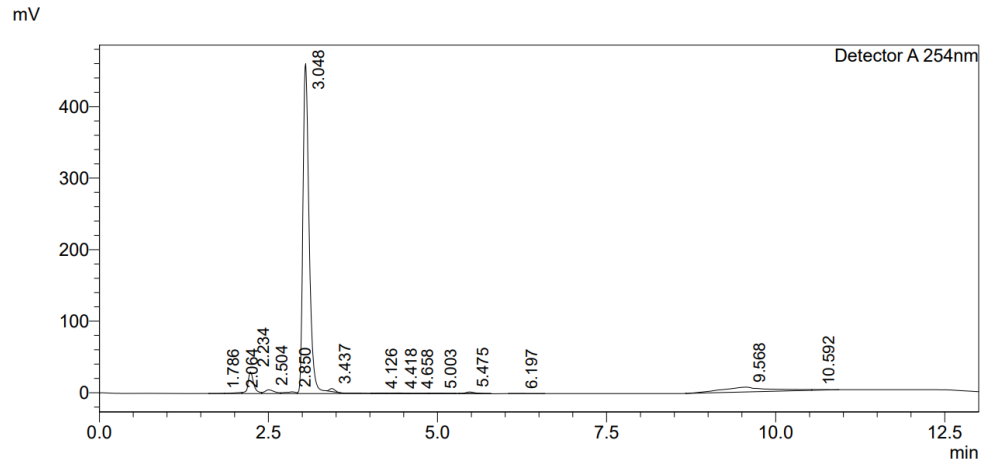


(y) Reaction with styrene $\mathbf{1}$ and diazoketone $\mathbf{6 n}$ o give cyclopropane $\mathbf{7 n}$ :

i. Chiral GC analysis of racemic standard (2 cis enantiomers + unresolved trans enantiomers):

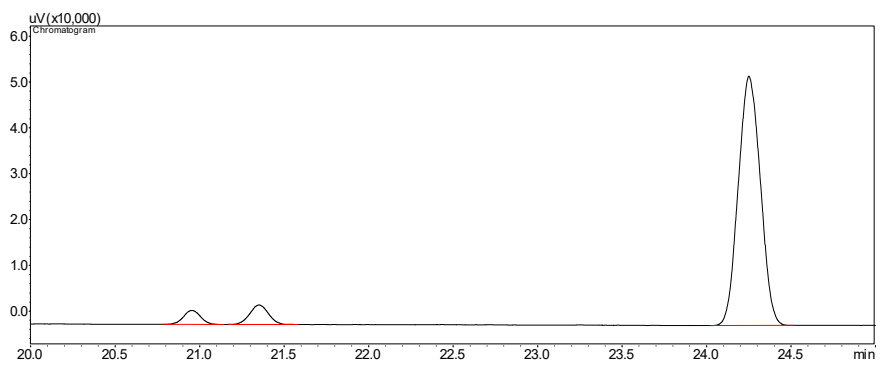

ii. Chiral GC analysis of $\mathbf{7 n}$ from $\mathrm{Mb}(\mathrm{H} 64 \mathrm{G}, \mathrm{V} 68 \mathrm{~A})$-catalyzed reaction:

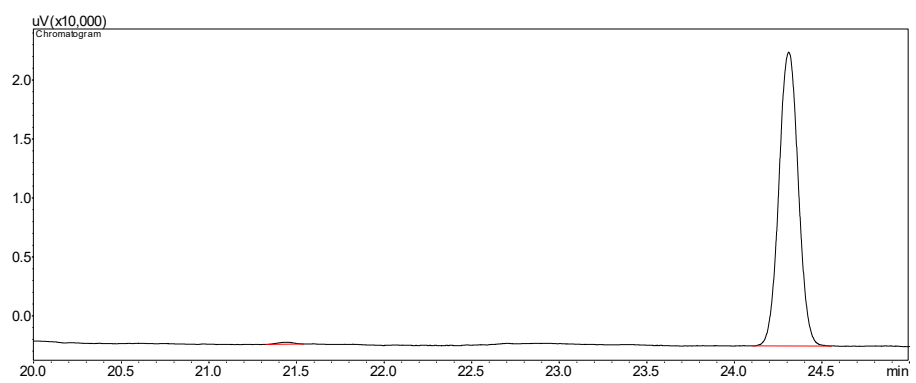

iii. Chiral HPLC analysis of racemic trans-7n (styrene starting material +2 trans enantiomers):

$\mathrm{mV}$

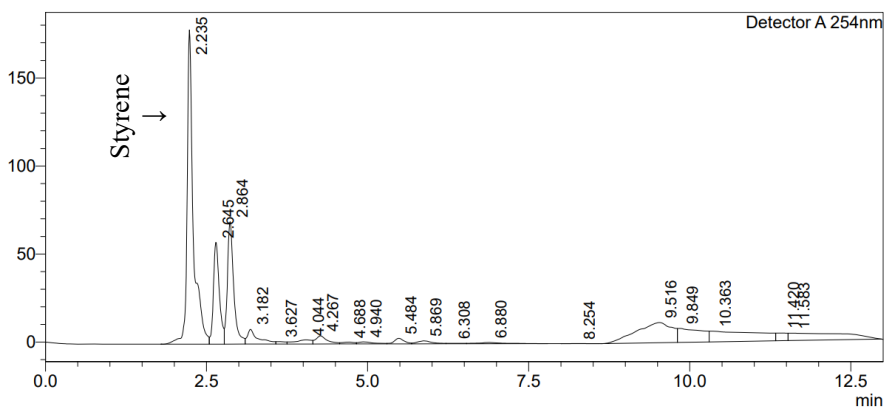

iv. Chiral HPLC analysis of $\mathbf{7 n}$ from Mb(H64G,V68A)-catalyzed reaction:

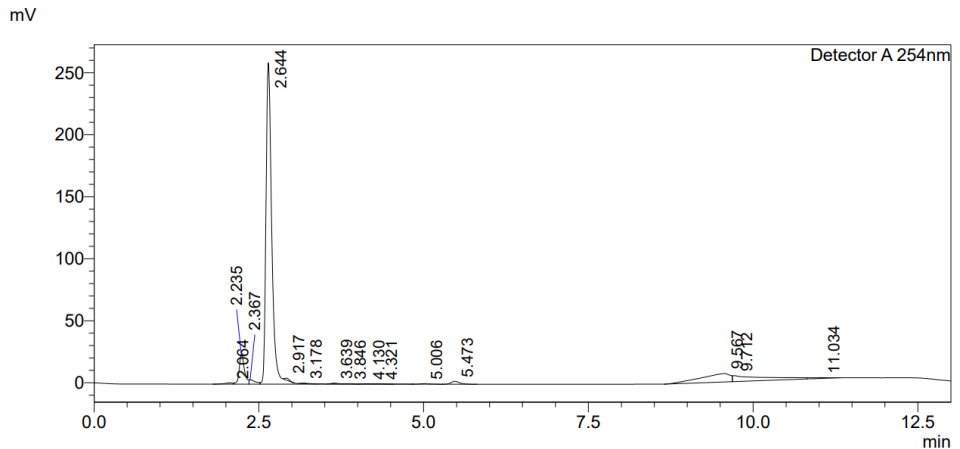


(z) Reaction with 4-methylstyrene 4a and diazoketone 6e to give cyclopropane 8a:

i. SFC analysis of racemic product ( 2 trans enantiomers +2 cis enantiomers):

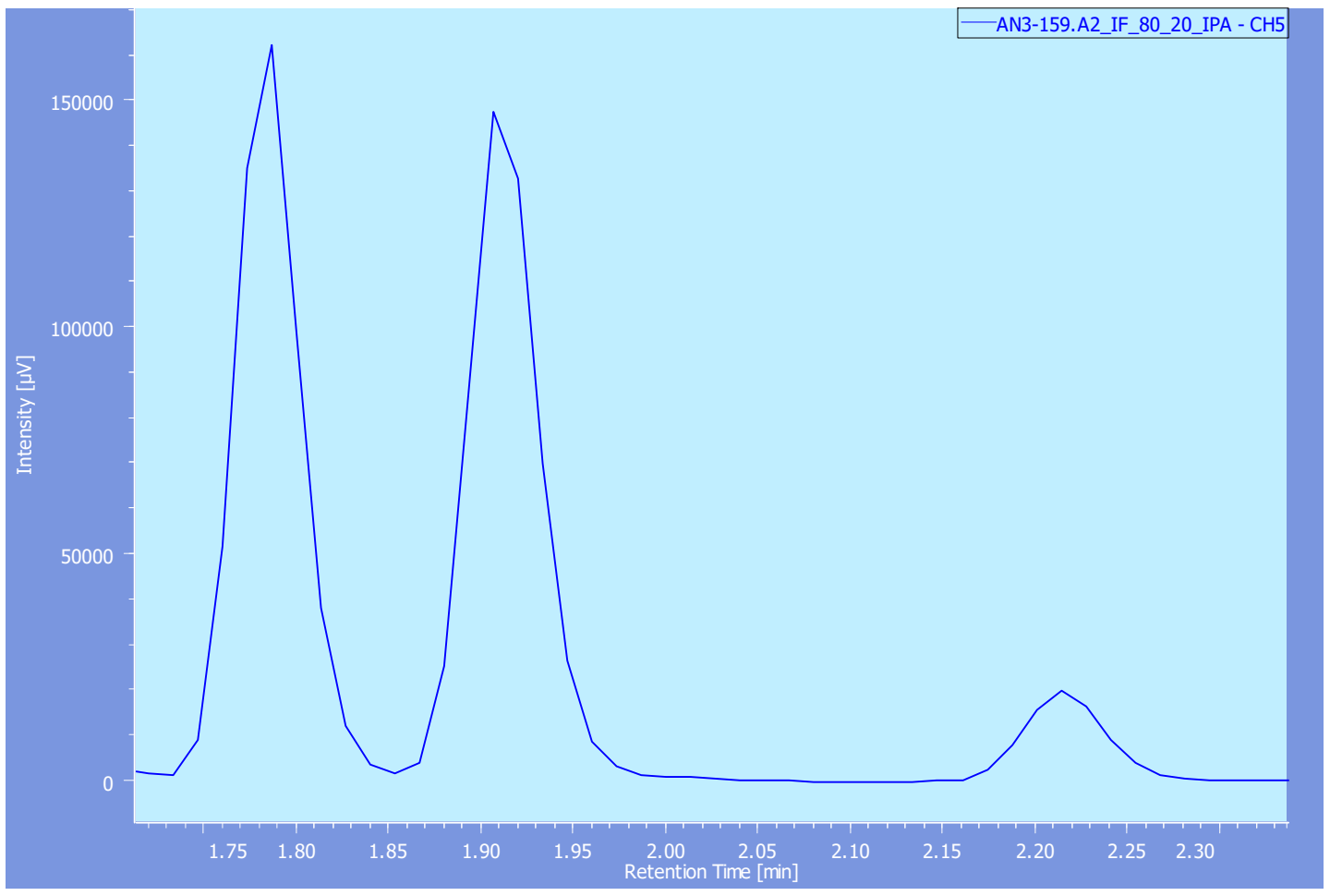

ii. $\mathrm{SFC}$ analysis of $\mathrm{Mb}(\mathrm{H} 64 \mathrm{G}, \mathrm{V} 68 \mathrm{~A})$-catalyzed reaction:

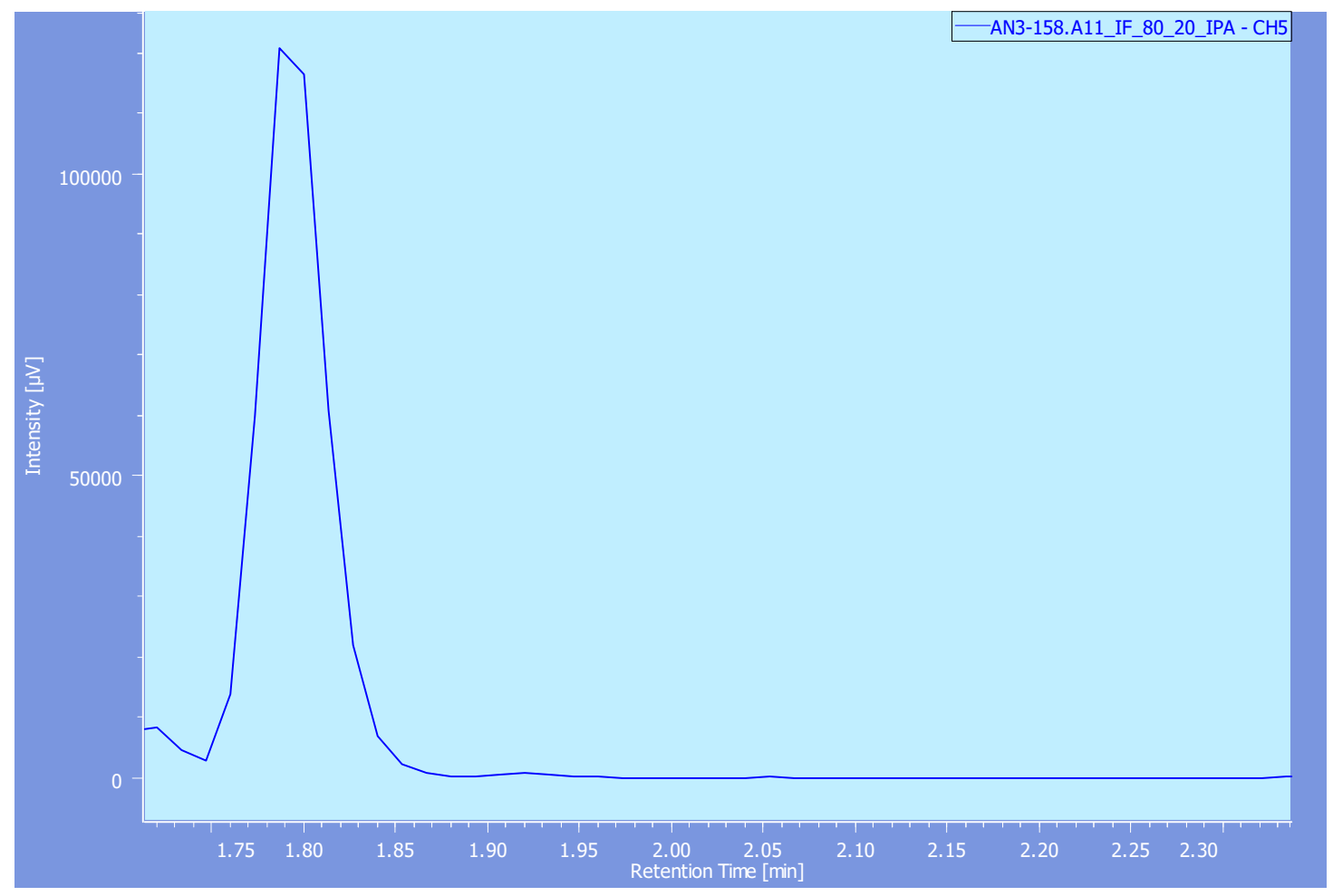


(aa) Reaction with 4-methoxystyrene $\mathbf{4 b}$ and diazoketone $\mathbf{6 e}$ to give cyclopropane $\mathbf{8 b}$ :

i. SFC analysis of racemic product ( 2 trans enantiomers +2 cis enantiomers):

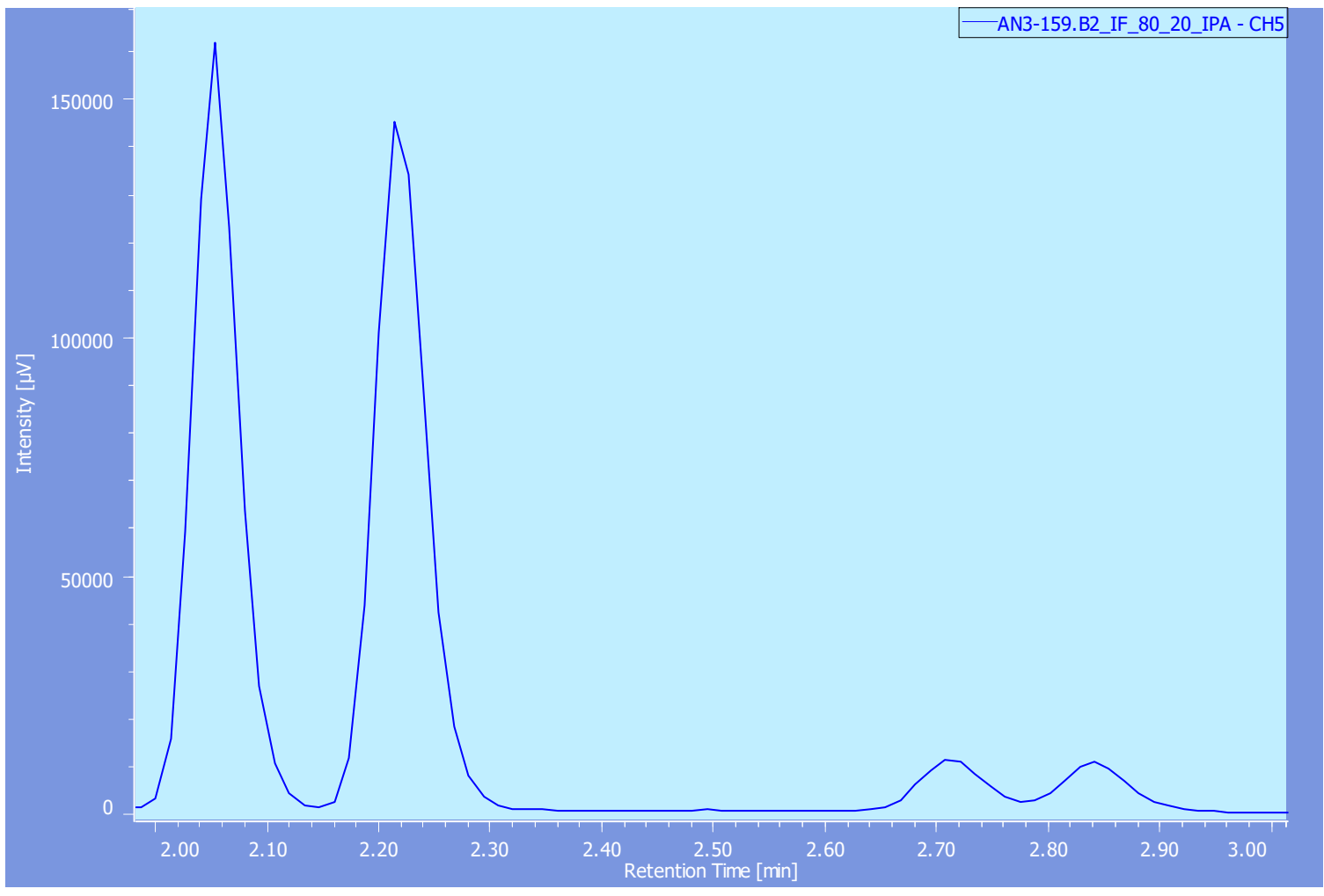

ii. $\mathrm{SFC}$ analysis of $\mathrm{Mb}(\mathrm{H} 64 \mathrm{G}, \mathrm{V} 68 \mathrm{~A})$-catalyzed reaction:

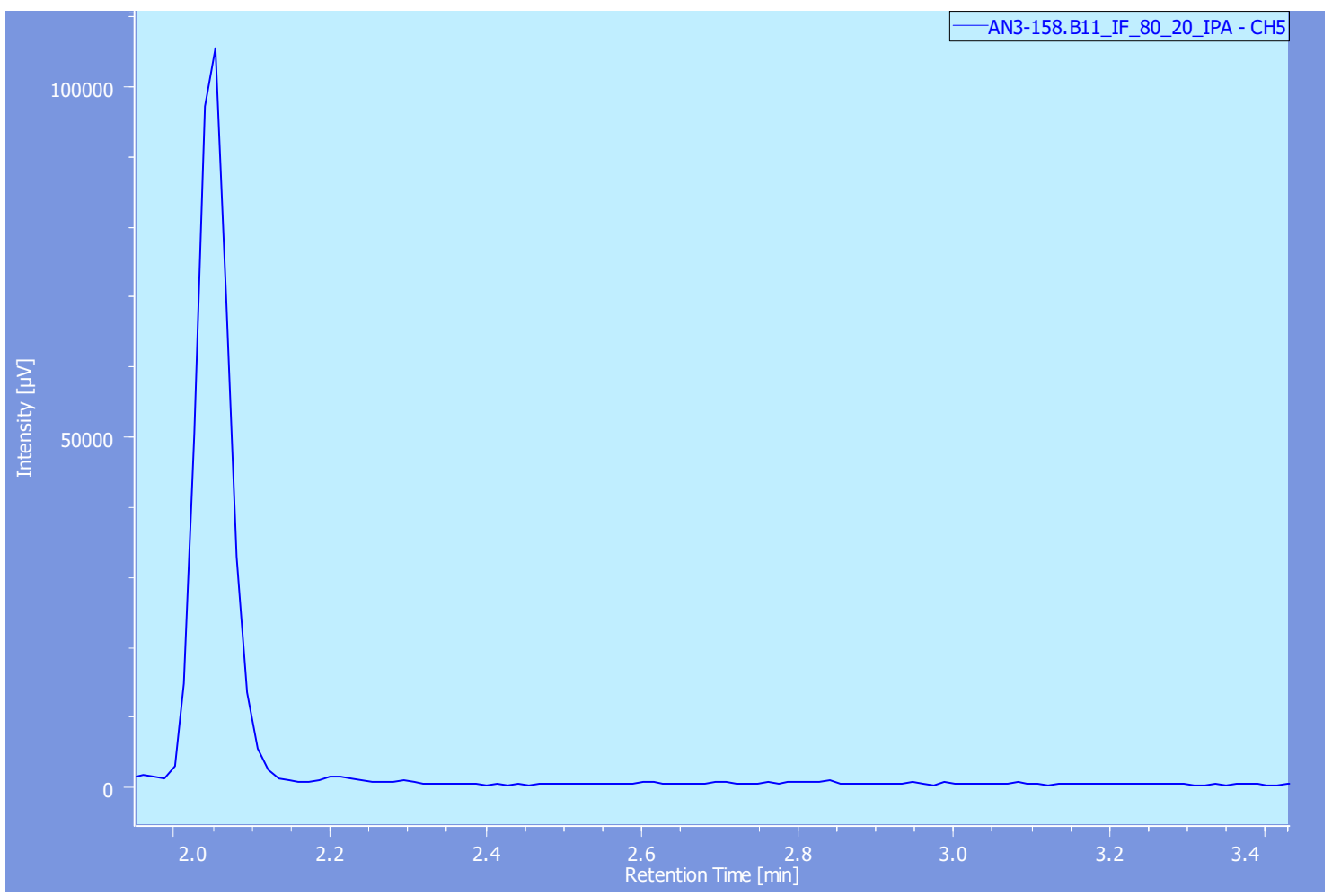


(bb) Reaction with 4-chlorostyrene $\mathbf{4 d}$ and diazoketone $\mathbf{6 e}$ to give cyclopropane $\mathbf{8 c}$ :

i. SFC analysis of racemic product ( 2 trans enantiomers +2 cis enantiomers):

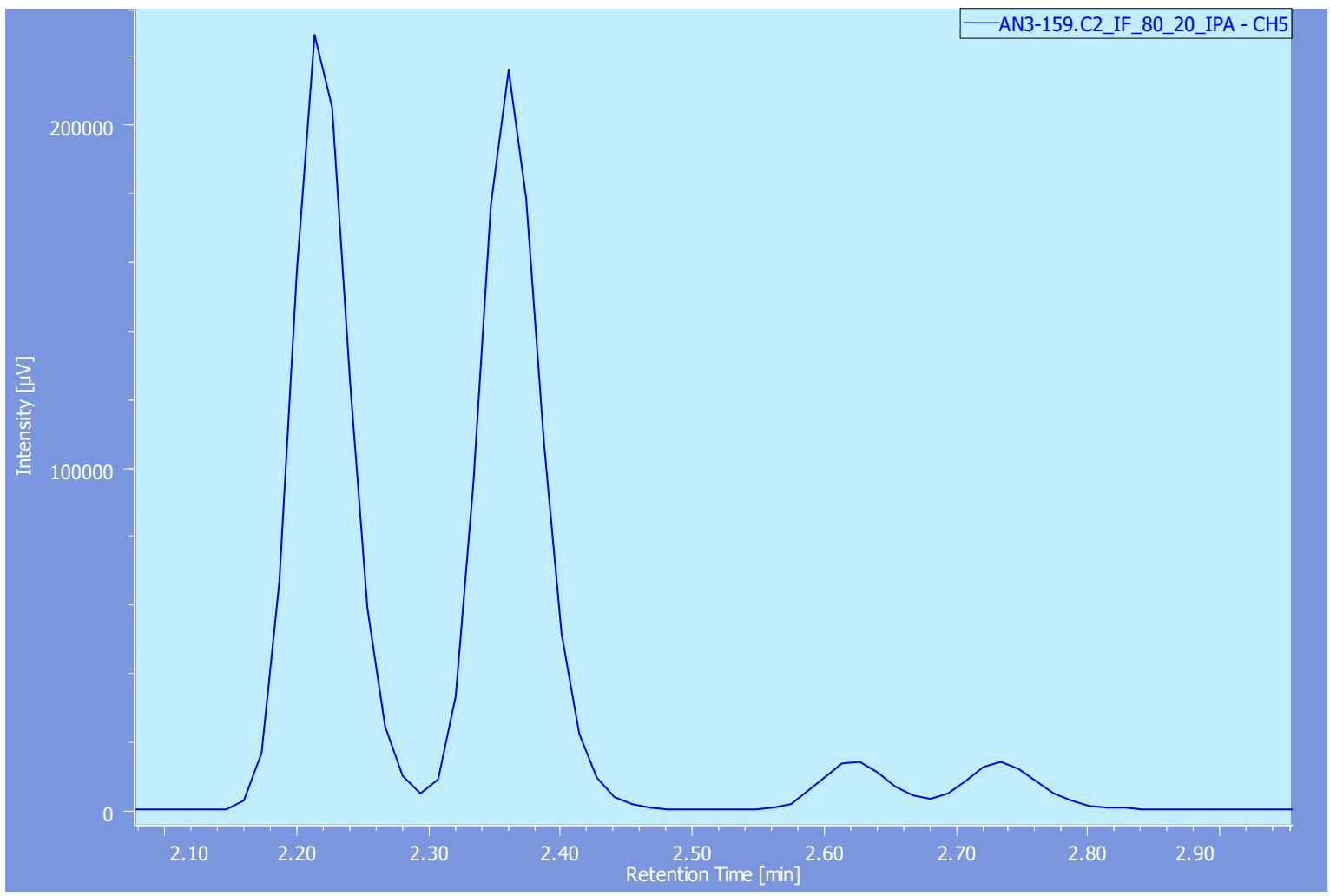

ii. SFC analysis of $\mathrm{Mb}(\mathrm{H} 64 \mathrm{G}, \mathrm{V} 68 \mathrm{~A})$-catalyzed reaction:

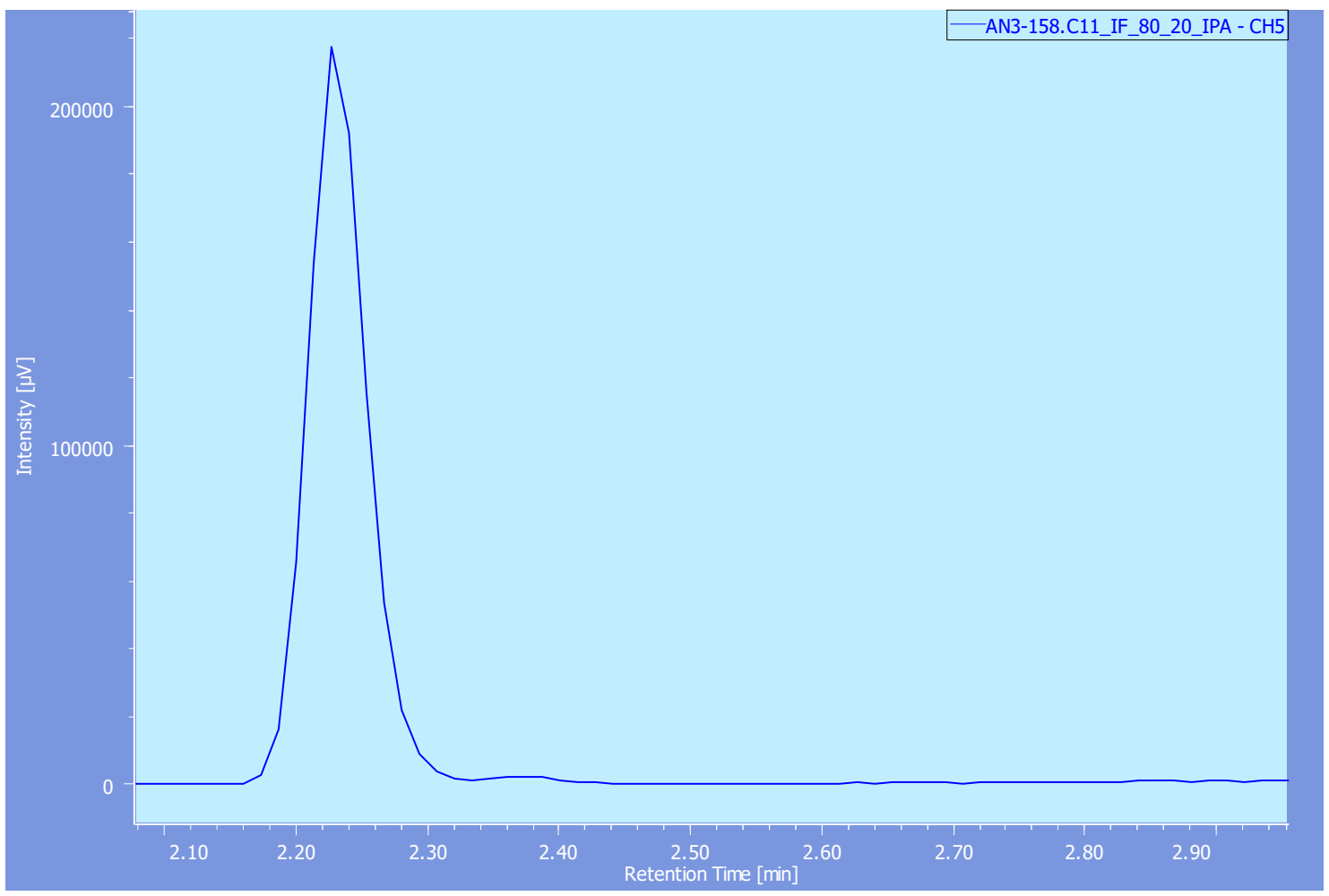


Figure S4. SFC chromatograms for determination of enantiomeric and diastereomeric excess in the chemical diversification of enzymatically produced keto-cyclopropane $\mathbf{3}$, as described in the experimental procedures. The racemic standards were prepared in the same manner starting from (racemic) trans-isomer of cyclopropane 3 .

(a) Reduction of 2-phenyl-1-((1S, 2S)-2-phenylcyclopropyl)ethan-1-one 3 to give 9:

i. SFC analysis of racemic 9 (4 stereoisomers):

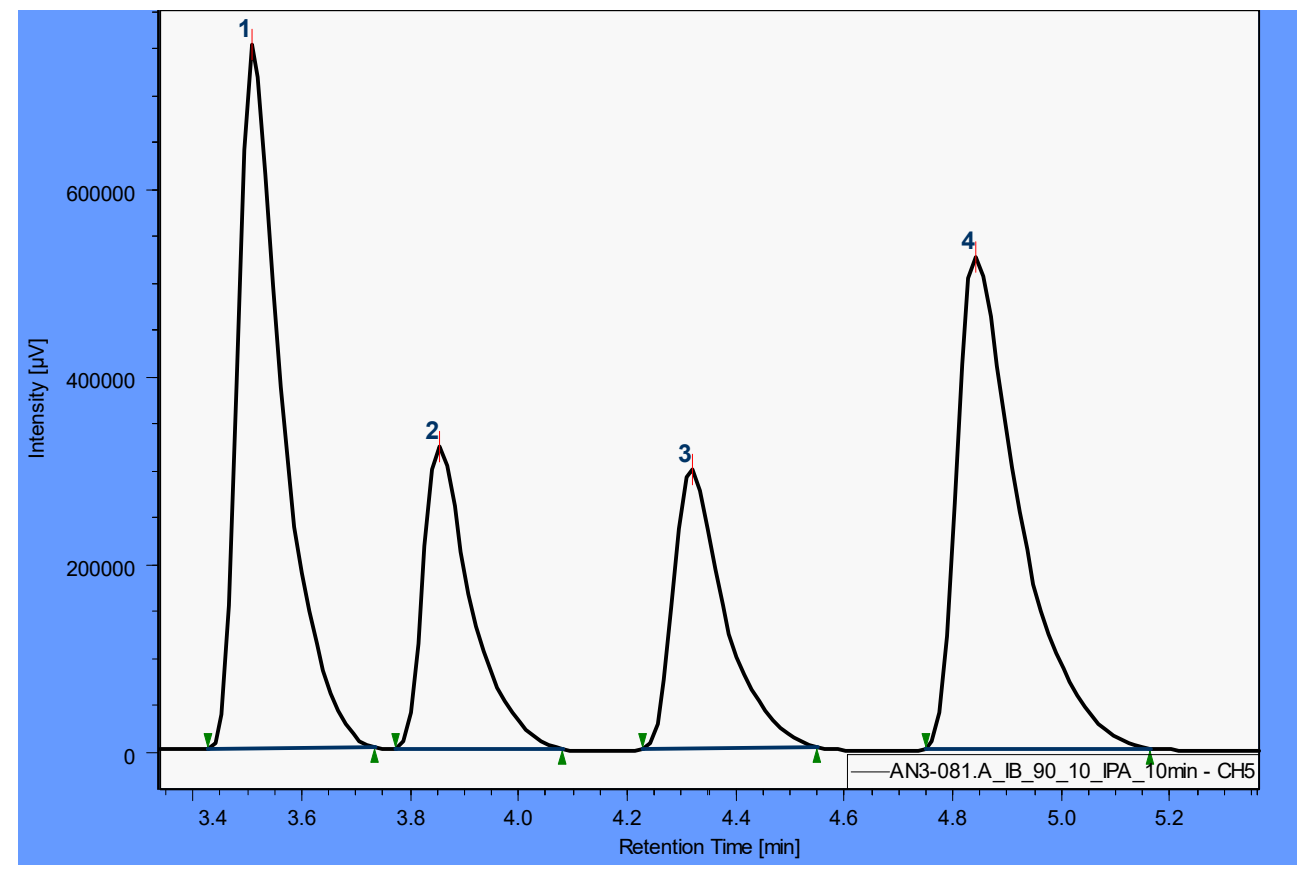

ii. SFC analysis of product $\mathbf{9}$ synthesized from enzymatically produced $\mathbf{3}$ :

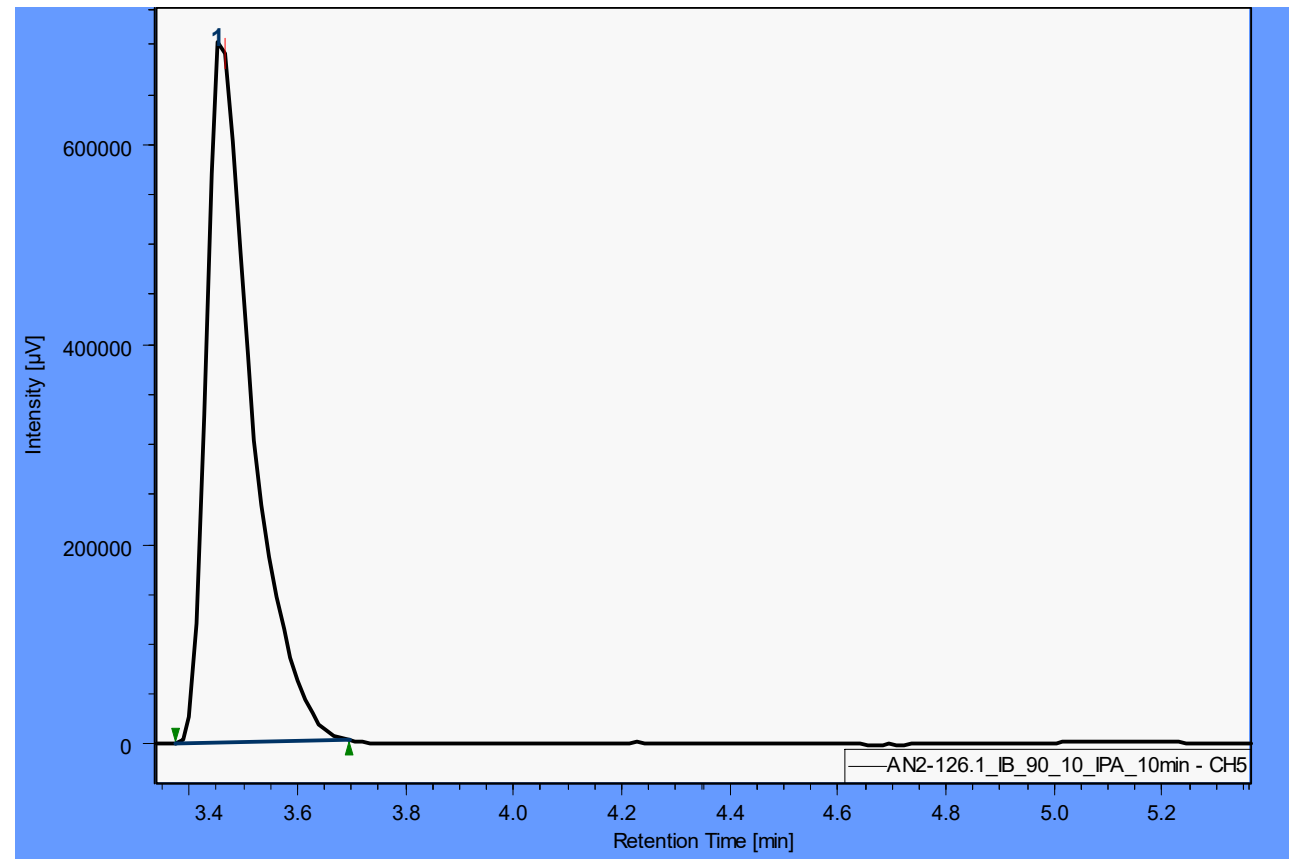


(b) Grignard reaction of 2-phenyl-1-((1S, 2S)-2-phenylcyclopropyl)ethan-1-one (3) to give 10: i. $\quad$ SFC analysis of racemic $\mathbf{1 0}$ (4 stereoisomers):

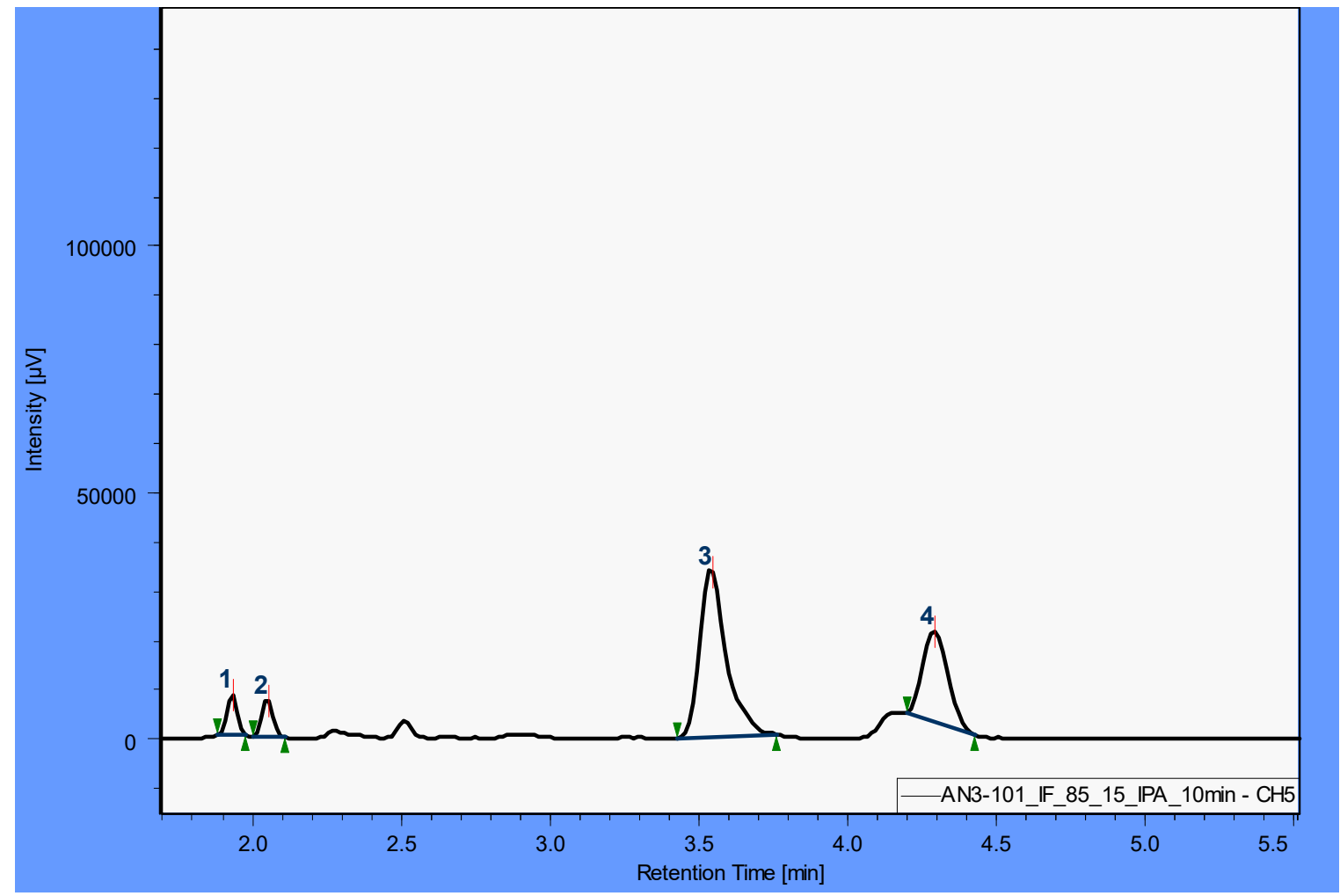

ii. SFC analysis of product $\mathbf{1 0}$ synthesized from enzymatically produced $\mathbf{3}$ :

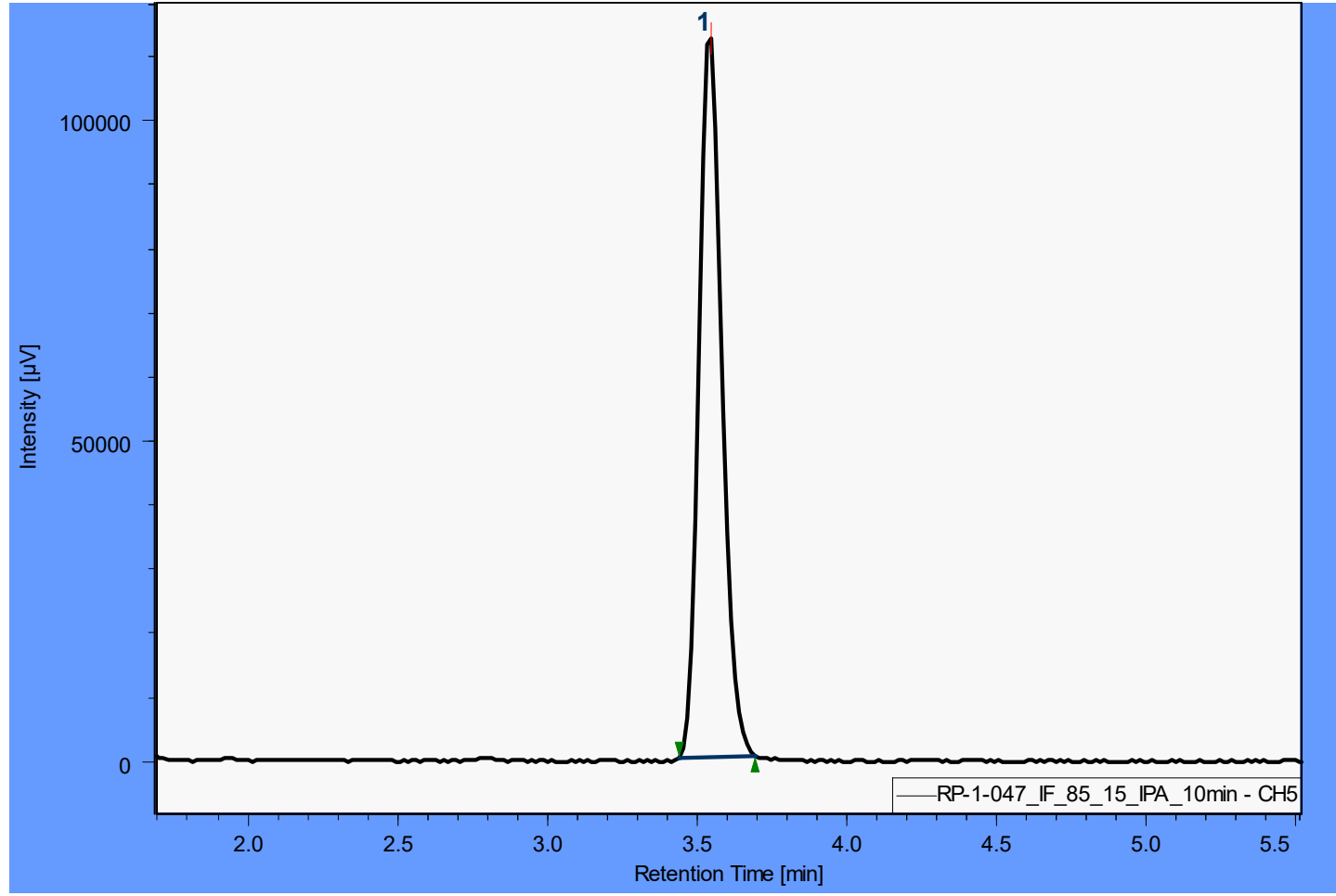


(c) Fluorination of 2-phenyl-1-((1S,2S)-2-phenylcyclopropyl)ethan-1-ol (9) to obtain 11:

i. SFC analysis of racemic $\mathbf{1 1}$ (4 stereoisomers):

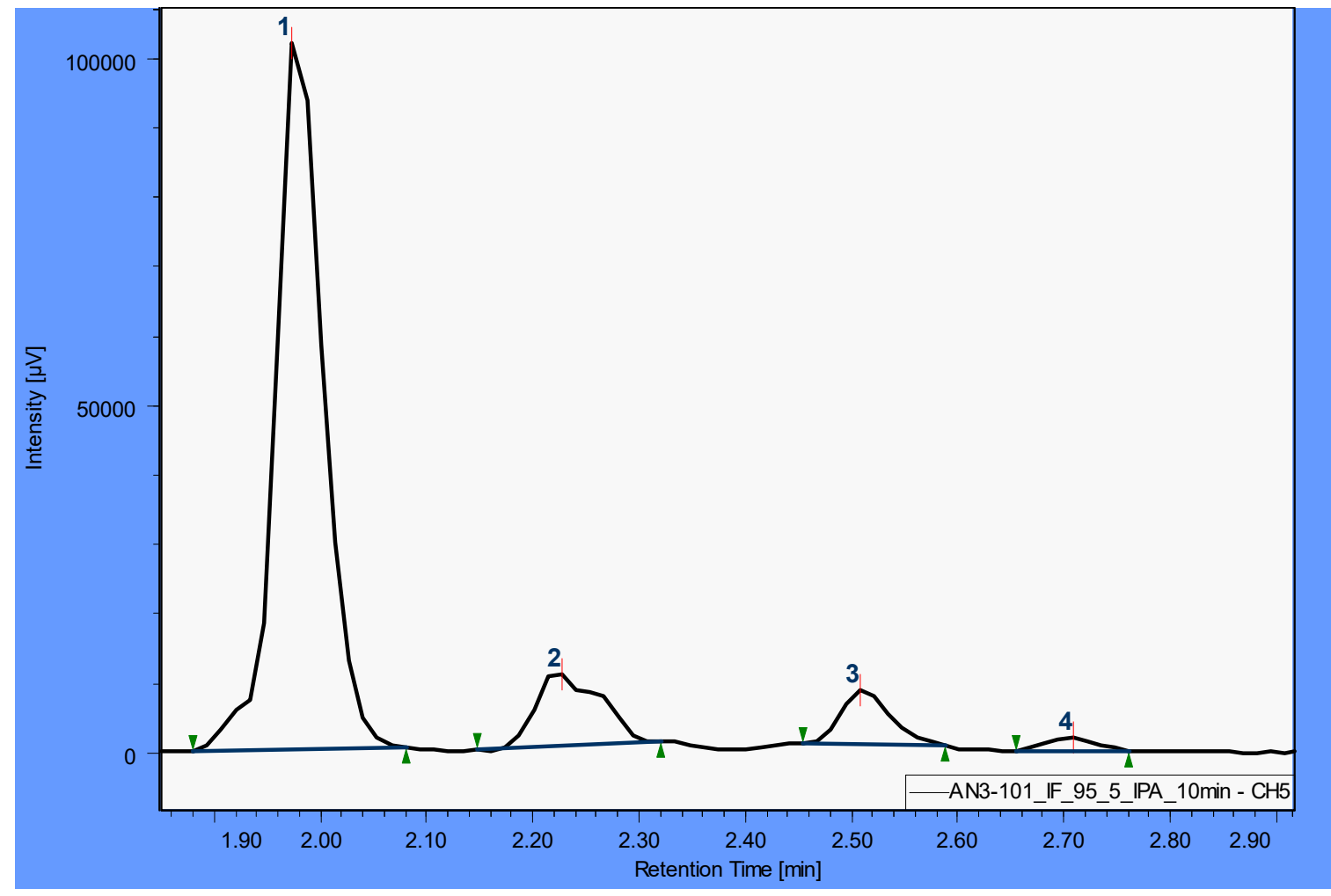

ii. SFC analysis of product $\mathbf{1 1}$ synthesized from chemoenzymatically produced $\mathbf{9}$ :

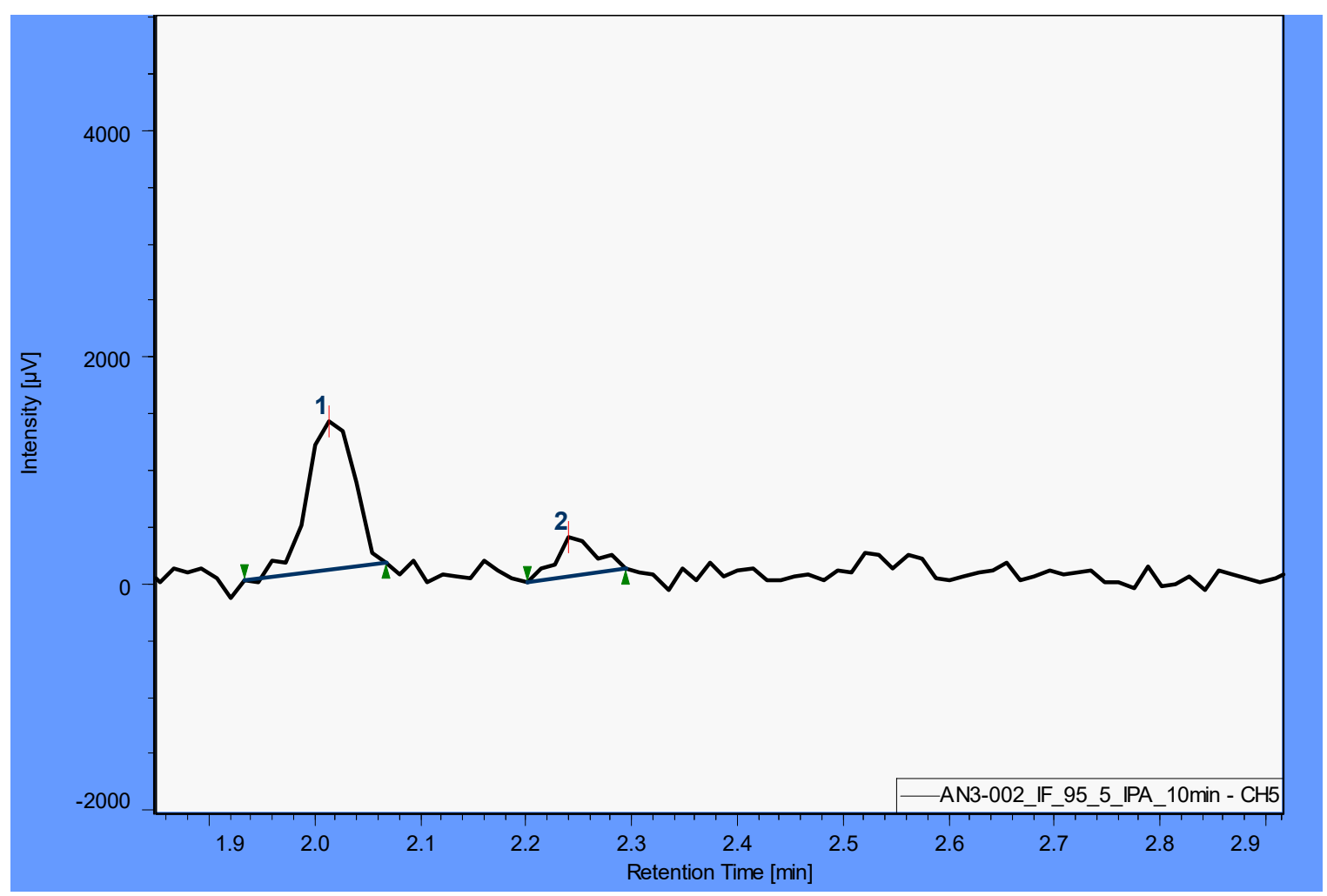


(d) Reductive amination of 2-phenyl-1-((1S, 2S)-2-phenylcyclopropyl)ethan-1-one (3) to give 12:

i. $\quad$ SFC analysis of racemic $\mathbf{1 2}$ (4 stereoisomers):

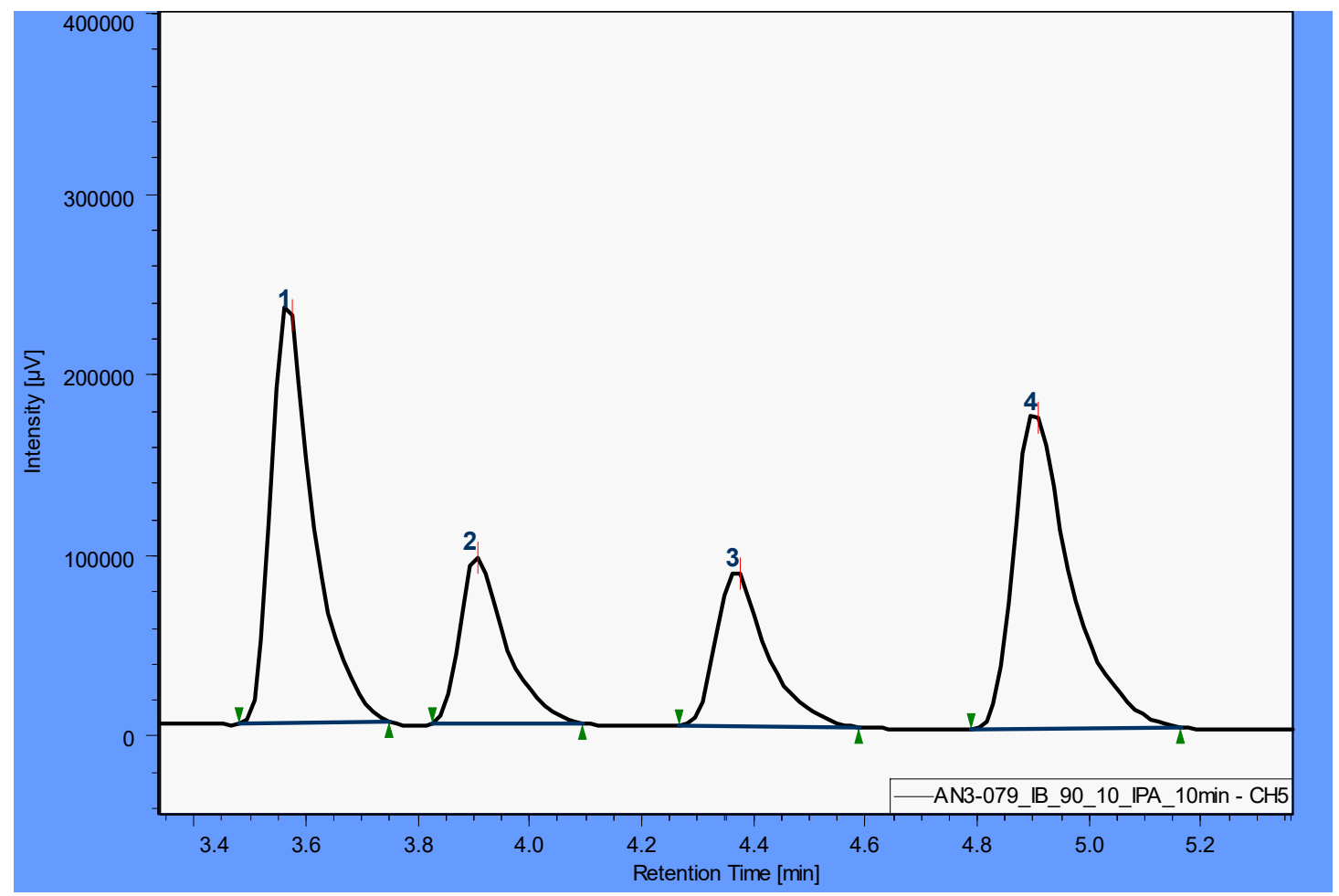

ii. SFC analysis of product $\mathbf{1 2}$ synthesized from enzymatically produced $\mathbf{3}$ :

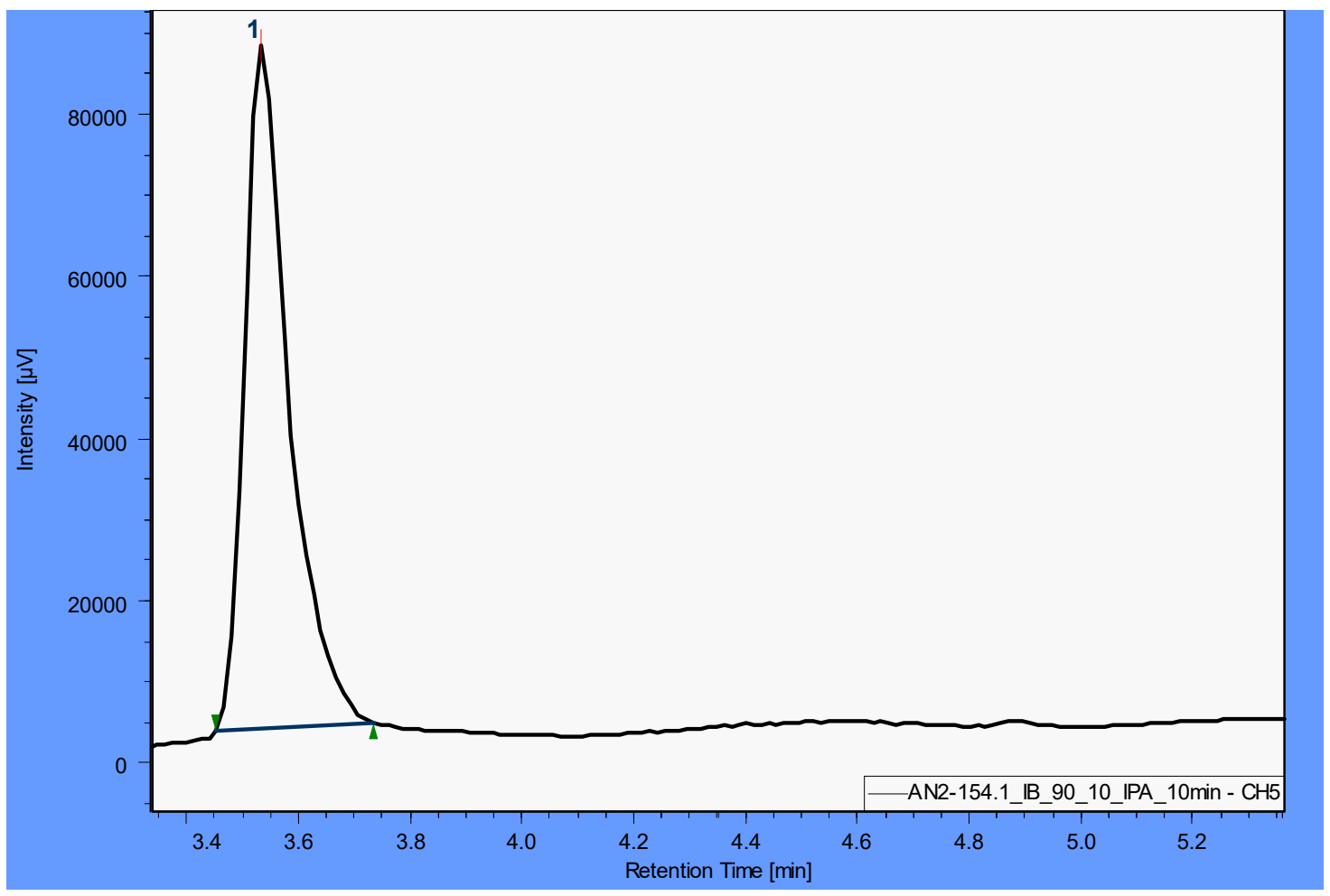


(e) $\alpha$-Methylation of 2-phenyl-1-((1S, 2S)-2-phenylcyclopropyl)ethan-1-one (3) to obtain 13:

i. SFC analysis of racemic $\mathbf{1 3}$ (4 stereoisomers):

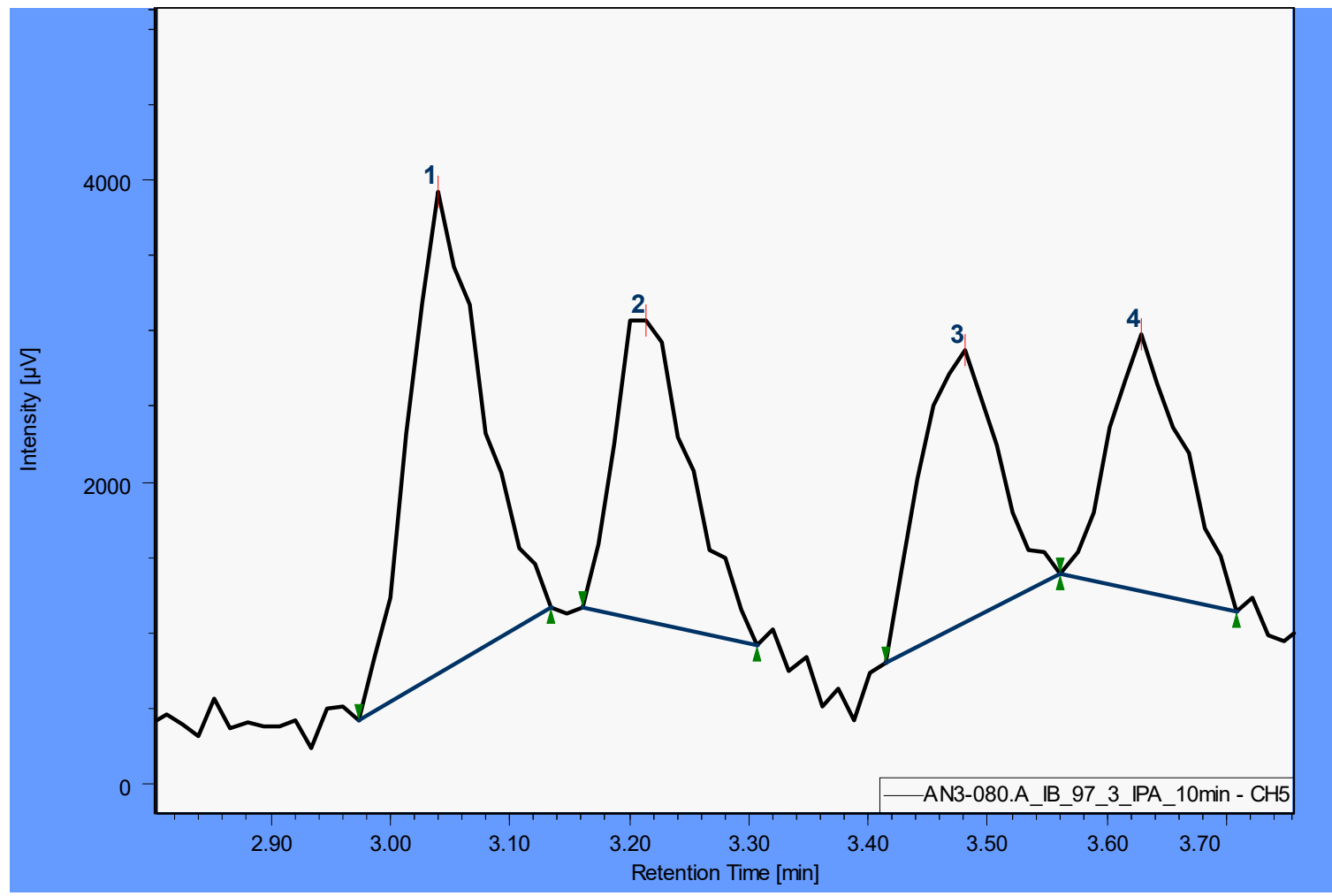

ii. $\quad$ SFC analysis of product $\mathbf{1 3}$ synthesized from enzymatically produced $\mathbf{3}$ :

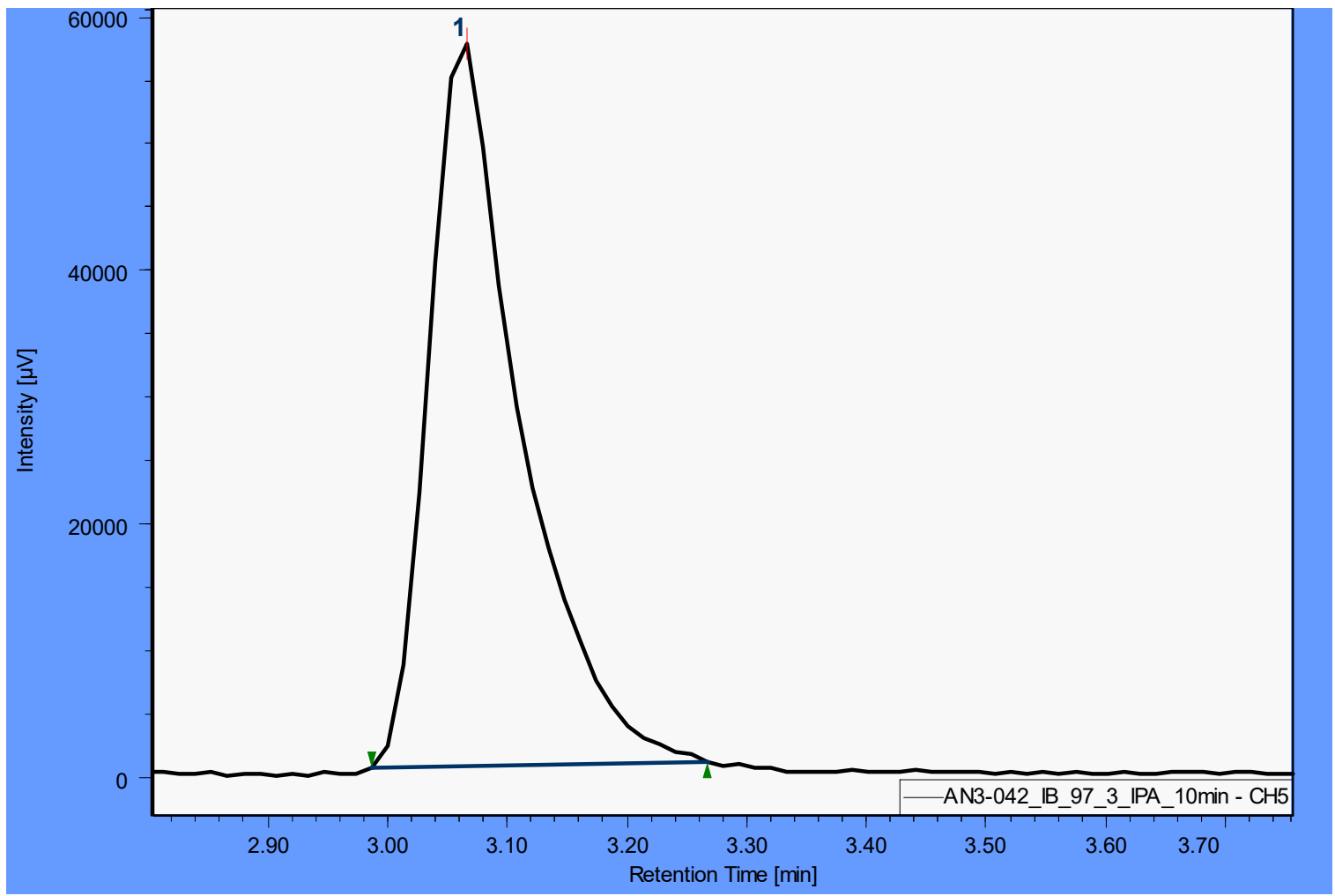


(f) $\alpha$-Fluorination of 2-phenyl-1-((1S, 2S)-2-phenylcyclopropyl)ethan-1-one (3) to obtain 14:

i. $\quad$ SFC analysis of racemic $\mathbf{1 4}$ (4 stereoisomers):

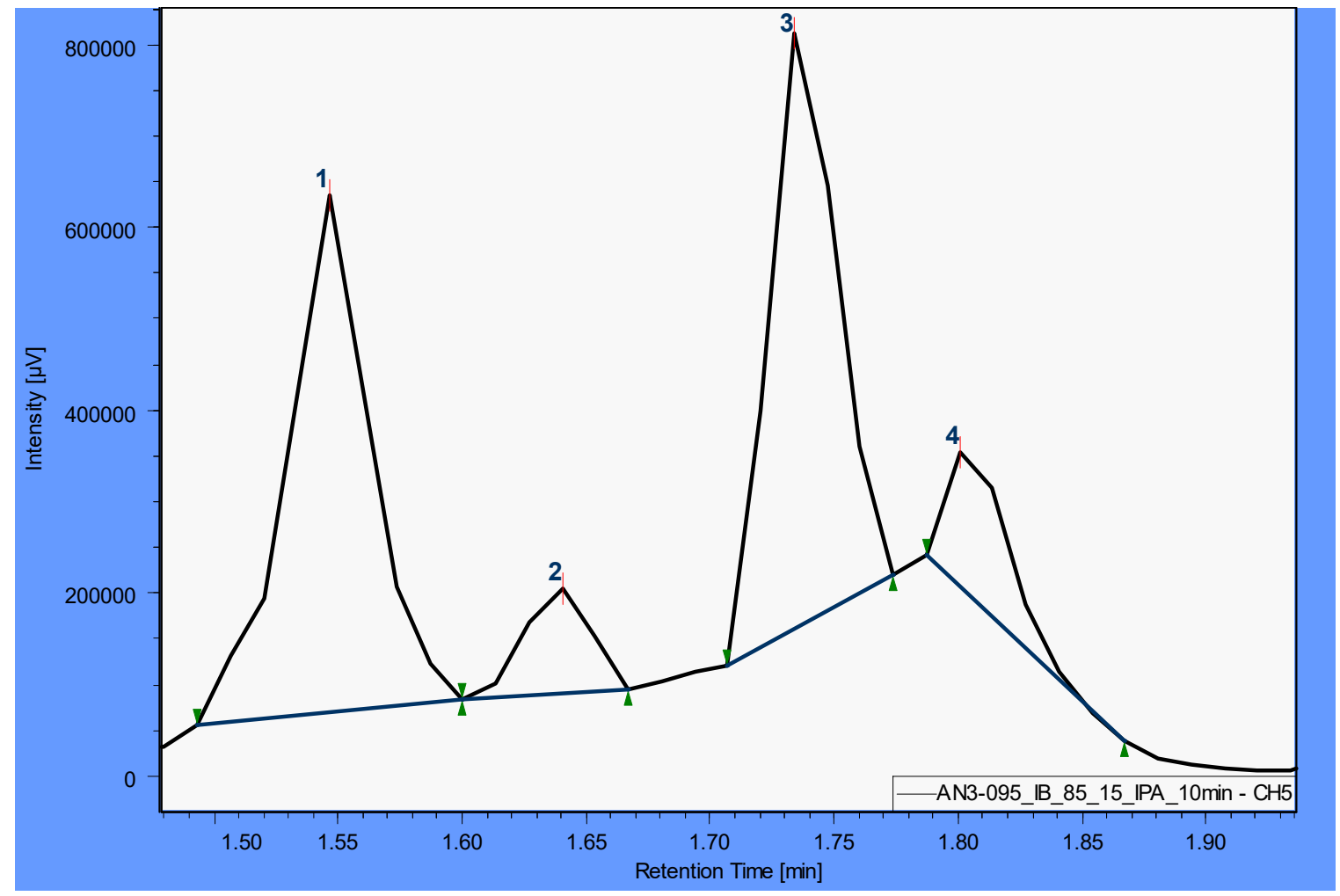

ii. SFC analysis of product $\mathbf{1 4}$ synthesized from enzymatically produced $\mathbf{3}$ :

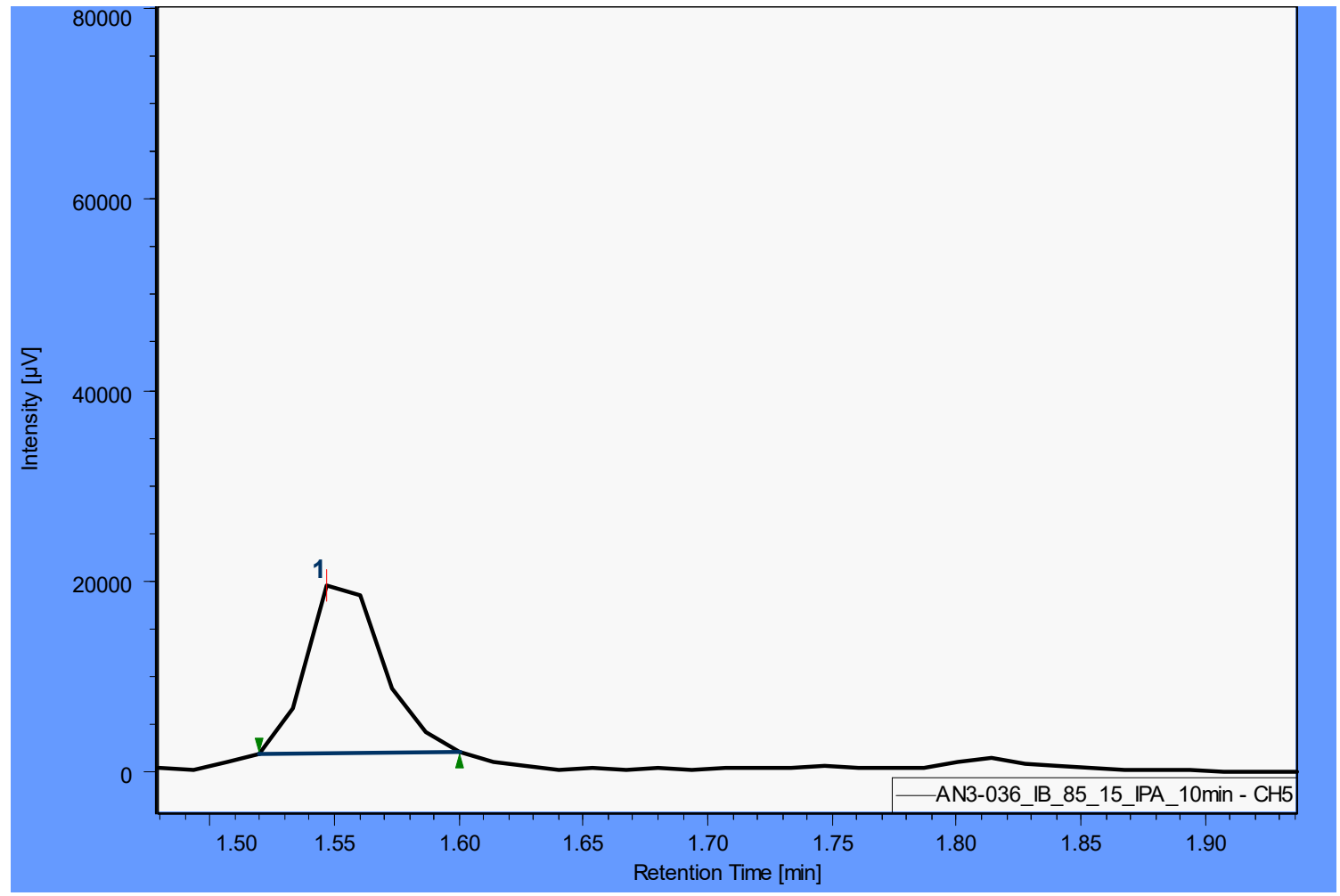


(g) Synthesis of diketone 15 from 2-phenyl-1-((1S, 2S)-2-phenylcyclopropyl)ethan-1-one (3): i. $\quad$ SFC analysis of racemic $\mathbf{1 5}$ (2 stereoisomers)::

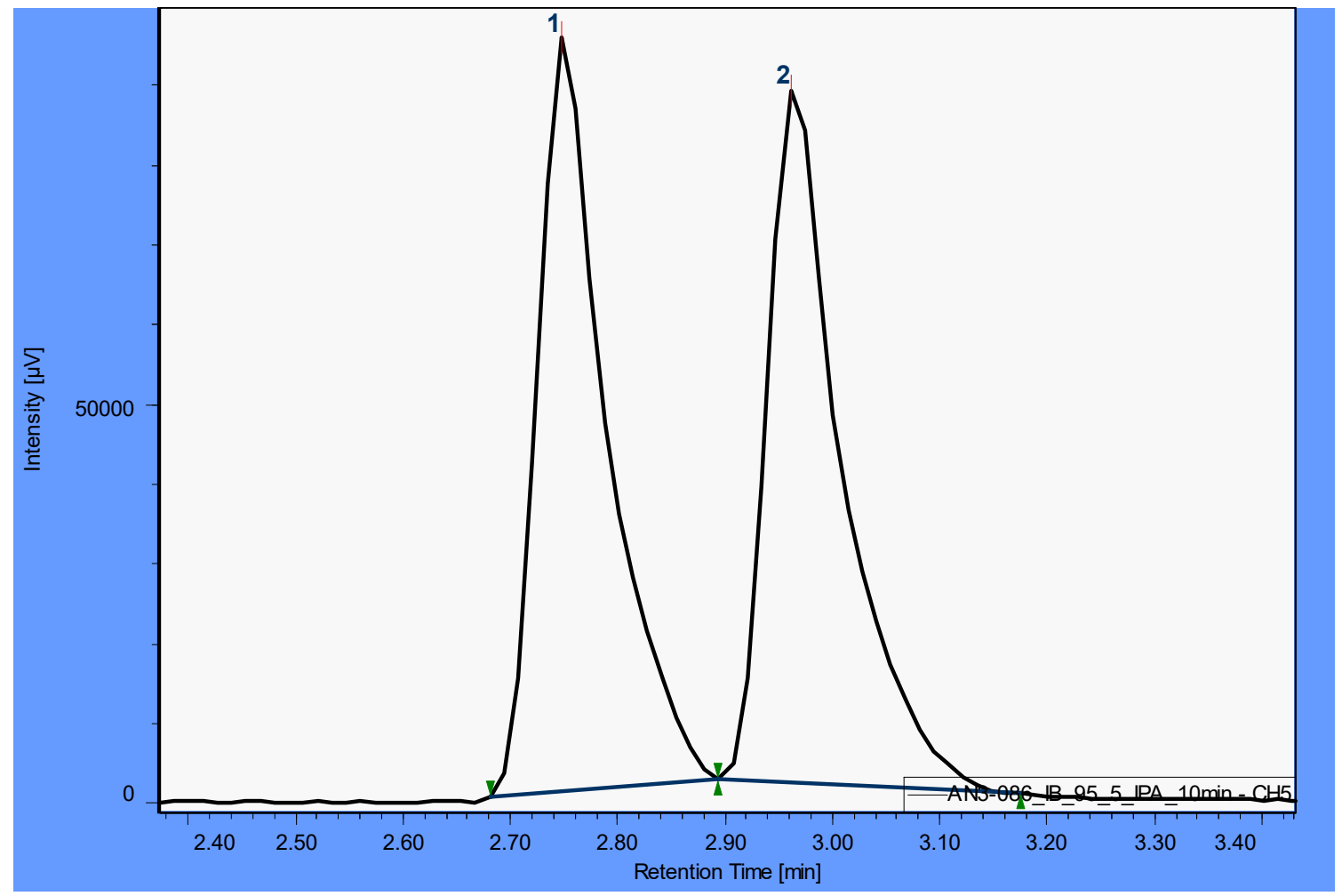

ii. SFC analysis of product $\mathbf{1 5}$ synthesized from enzymatically produced $\mathbf{3}$ :

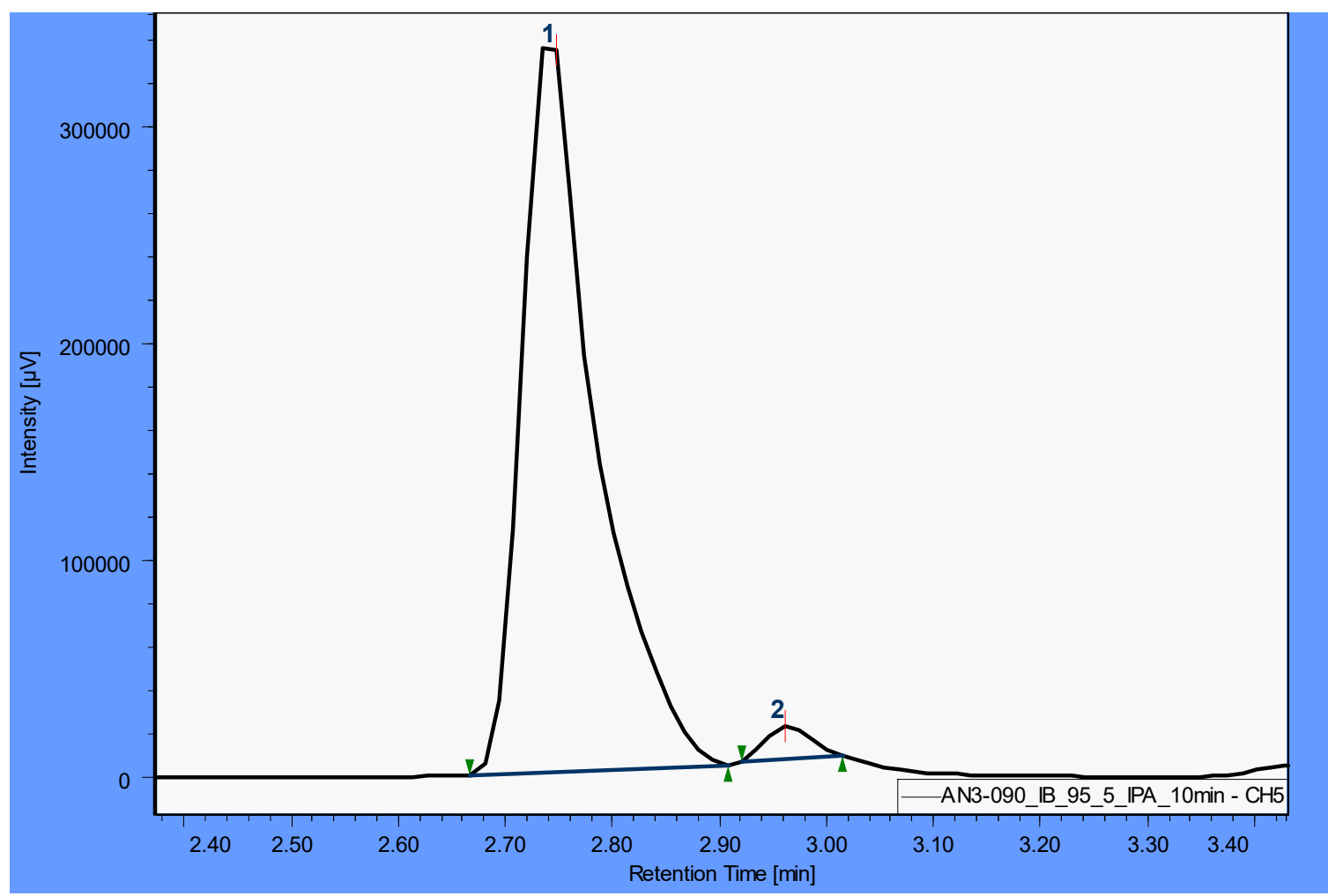


(h) Synthesis of enamine 16 from 2-phenyl-1-((1S, 2S)-2-phenylcyclopropyl)ethan-1-one (3):

i. $\quad$ SFC analysis of racemic $\mathbf{1 6}$ (2 stereoisomers):

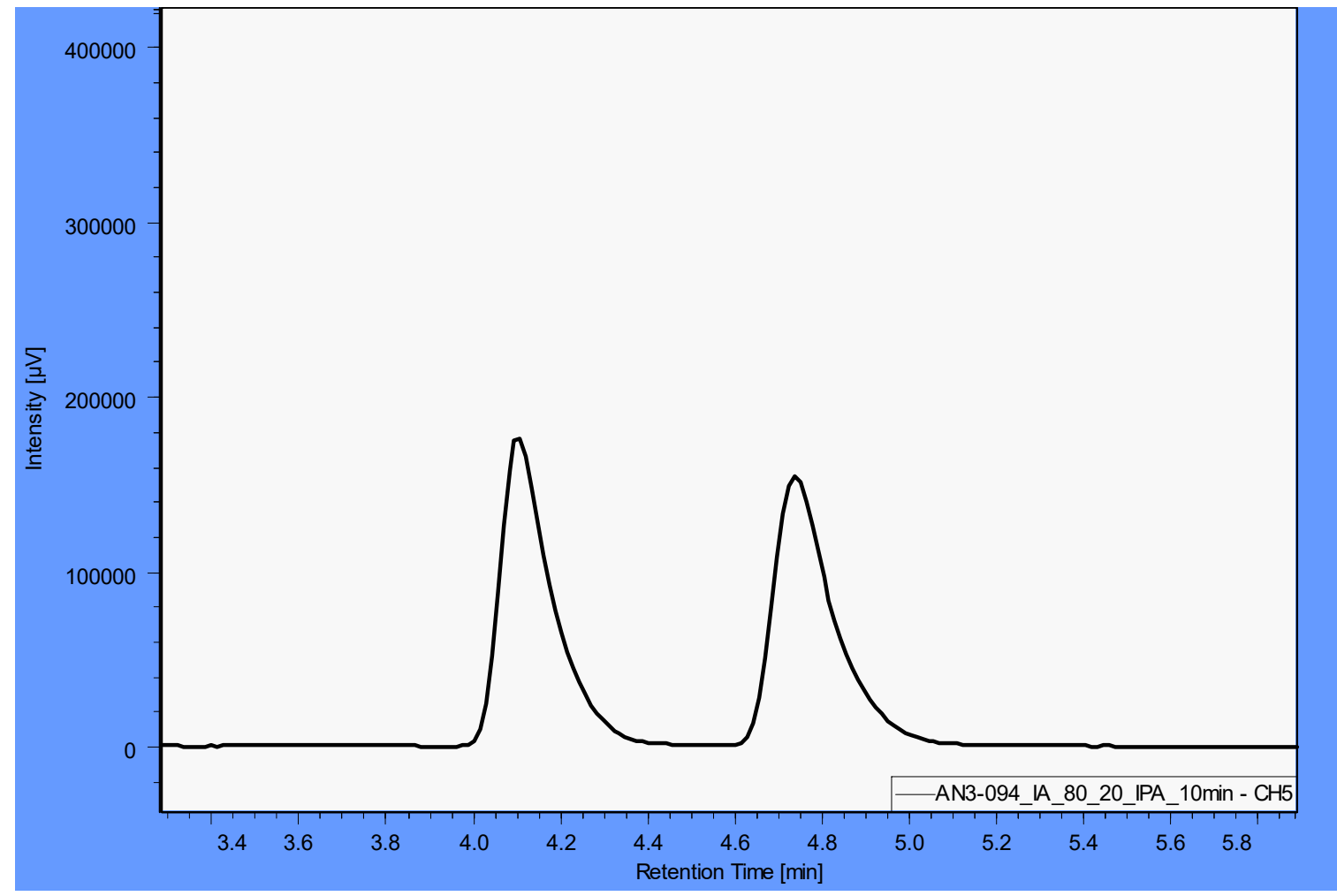

ii. SFC analysis of product $\mathbf{1 6}$ synthesized from enzymatically produced 3:

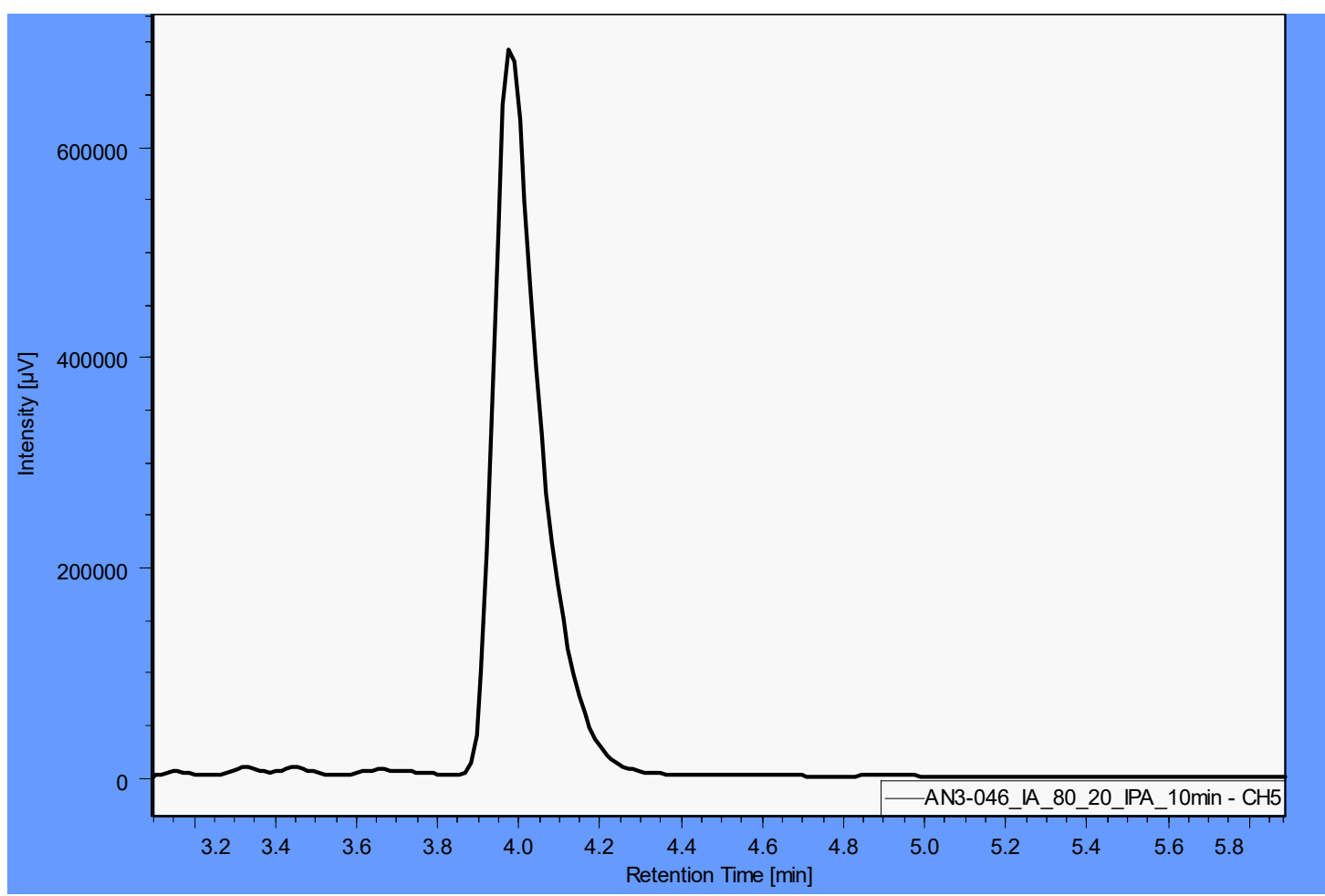


(i) Synthesis of silyl ether 17 from 2-phenyl-1-((1S, 2S)-2-phenylcyclopropyl)ethan-1-one (3): i. $\quad$ SFC analysis of racemic $\mathbf{1 7}$ (2 stereoisomers):

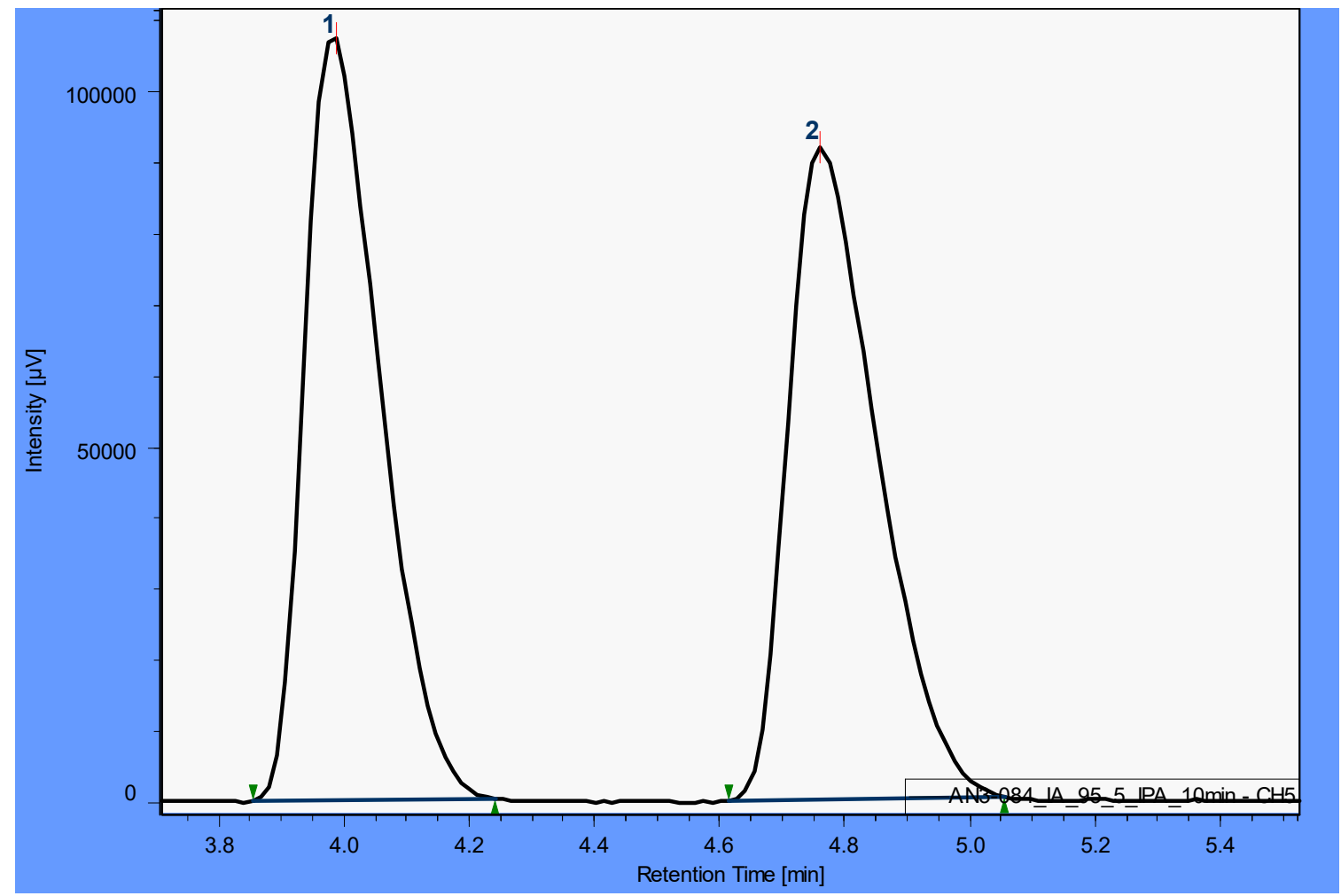

ii. SFC analysis of product synthesized from enzymatically produced $\mathbf{3}$ :

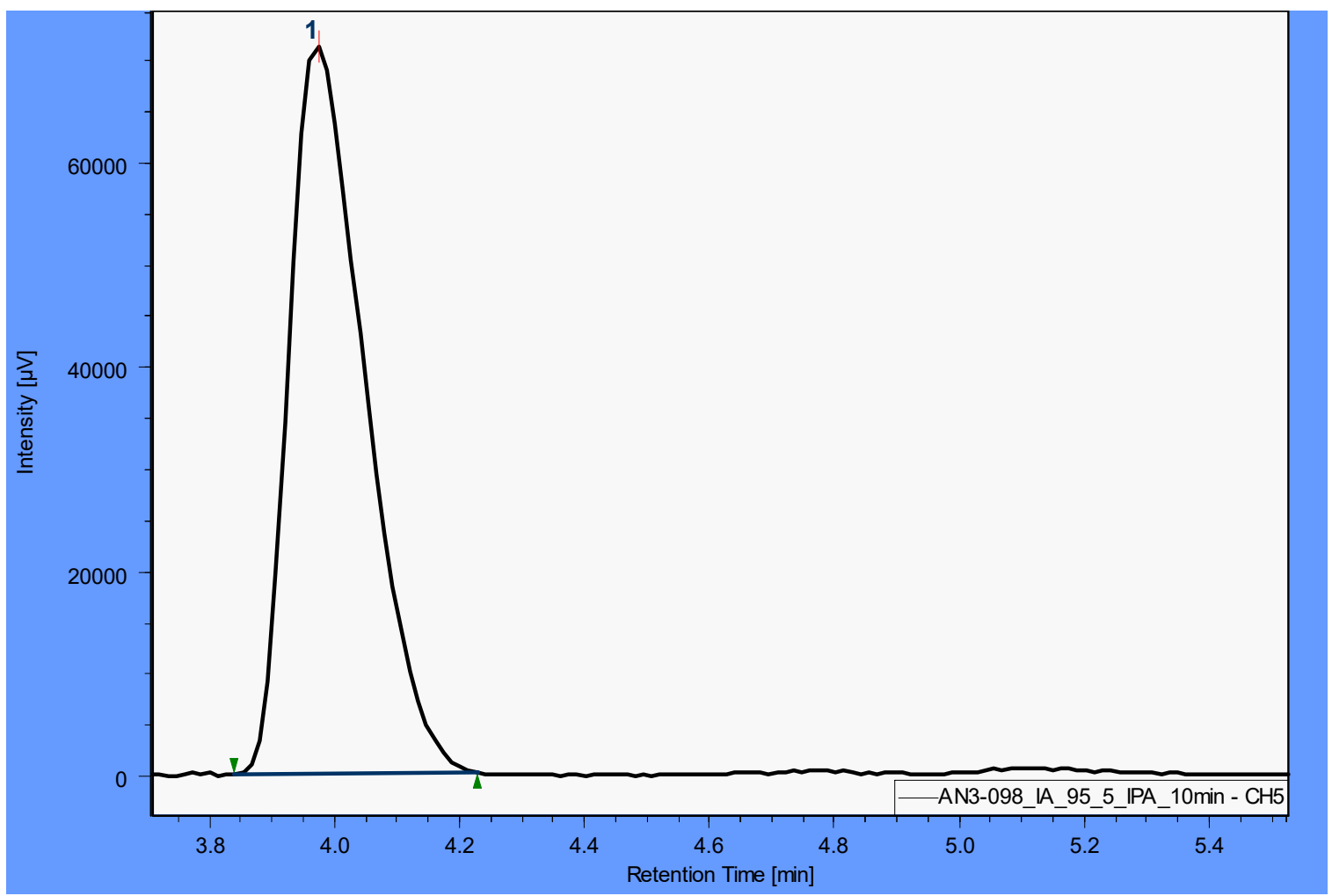


(j) Synthesis of quinoxaline 18 from 2-phenyl-1-((1S, 2S)-2-phenylcyclopropyl)ethan-1-one (3):

i. $\quad$ SFC analysis of racemic $\mathbf{1 8}$ (4 stereoisomers):

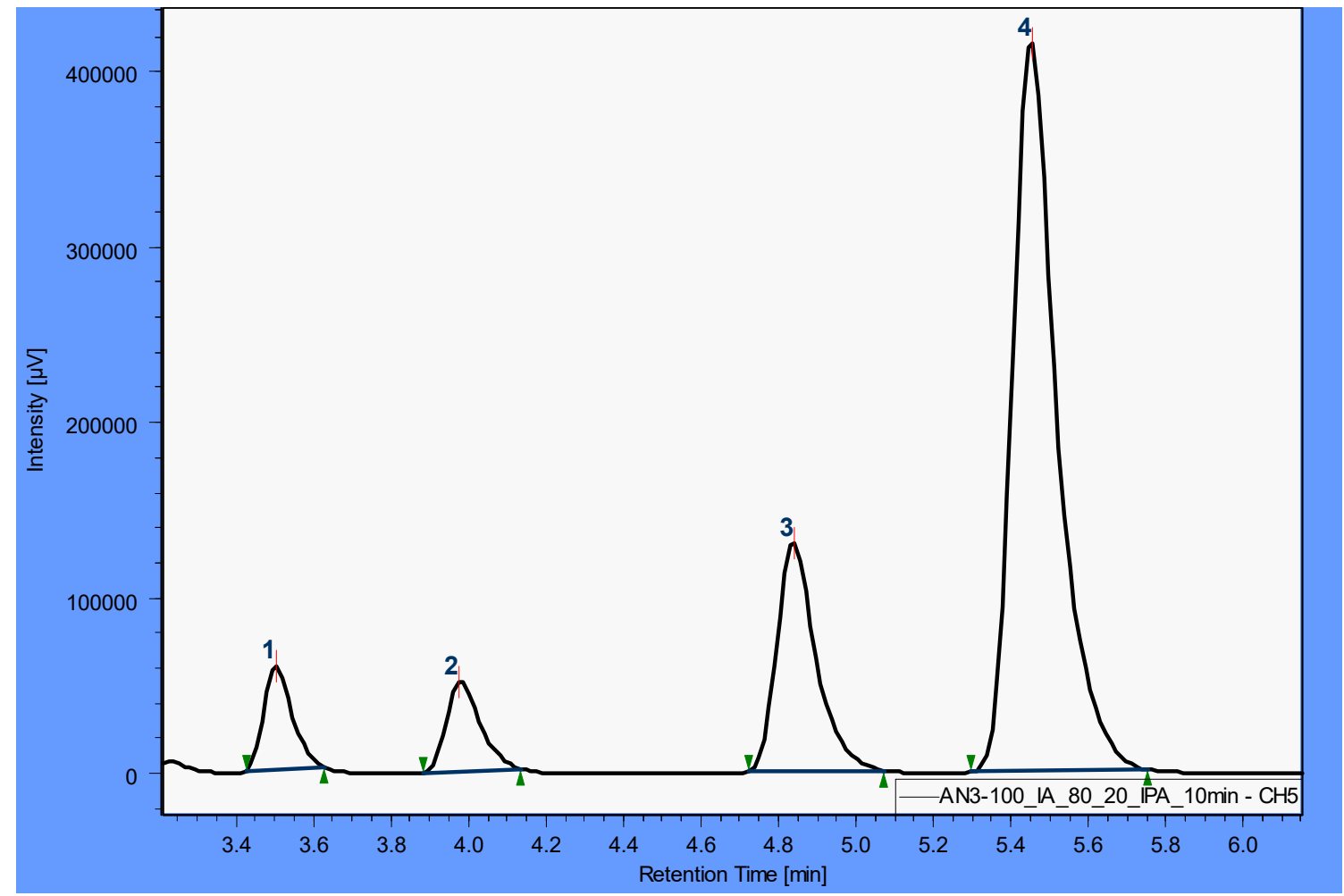

ii. $\quad$ SFC analysis of product $\mathbf{1 8}$ synthesized from enzymatically produced $\mathbf{3}$ :

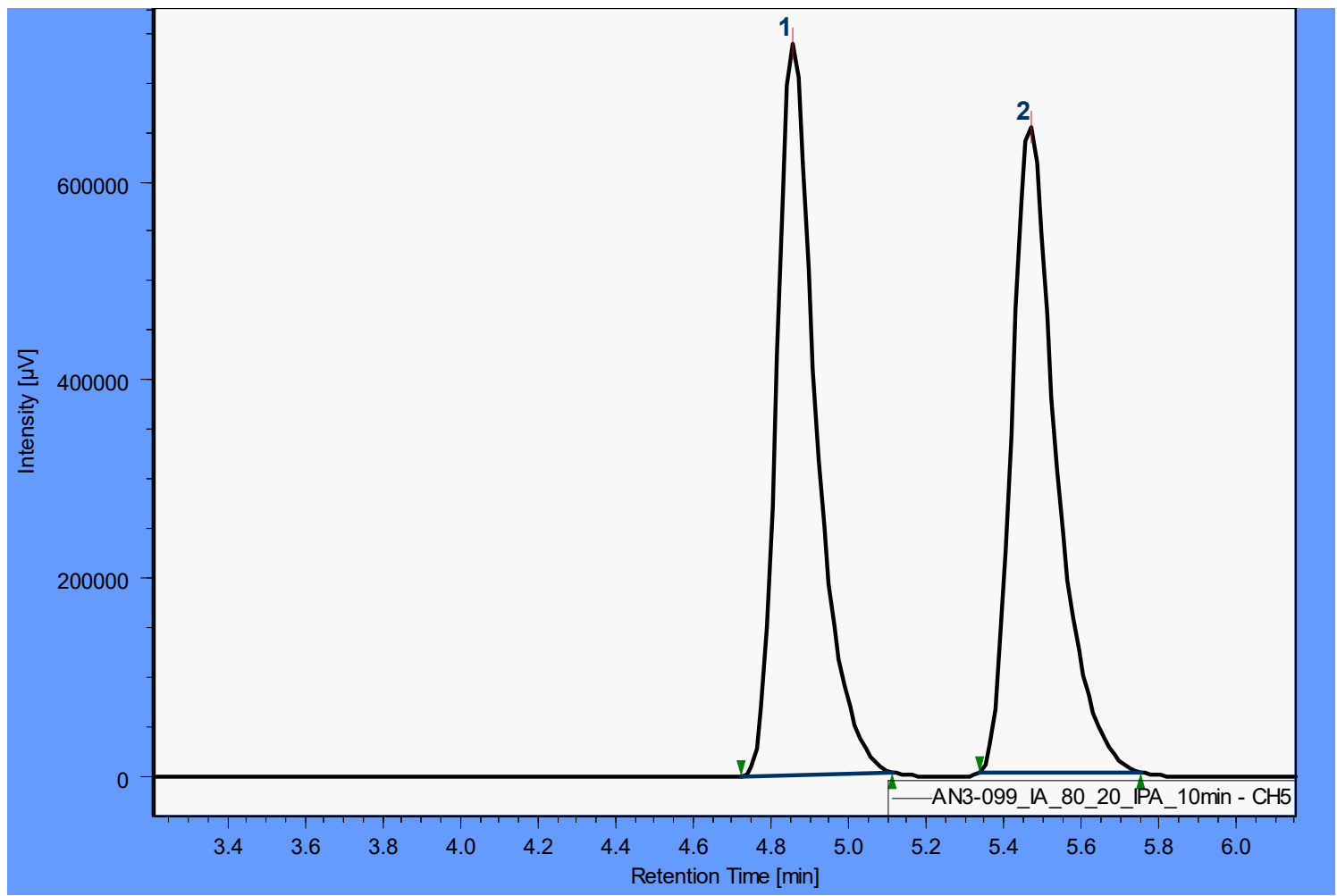


Figure S5. ORTEP of 1-(4-bromo-phenyl)-3-diazo-propan-2-one 5d with ellipsoids drawn at the $50 \%$ probability level. Hydrogen atoms were located in the difference Fourier map and refined freely. They are represented here as spheres of arbitrary radius for clarity.

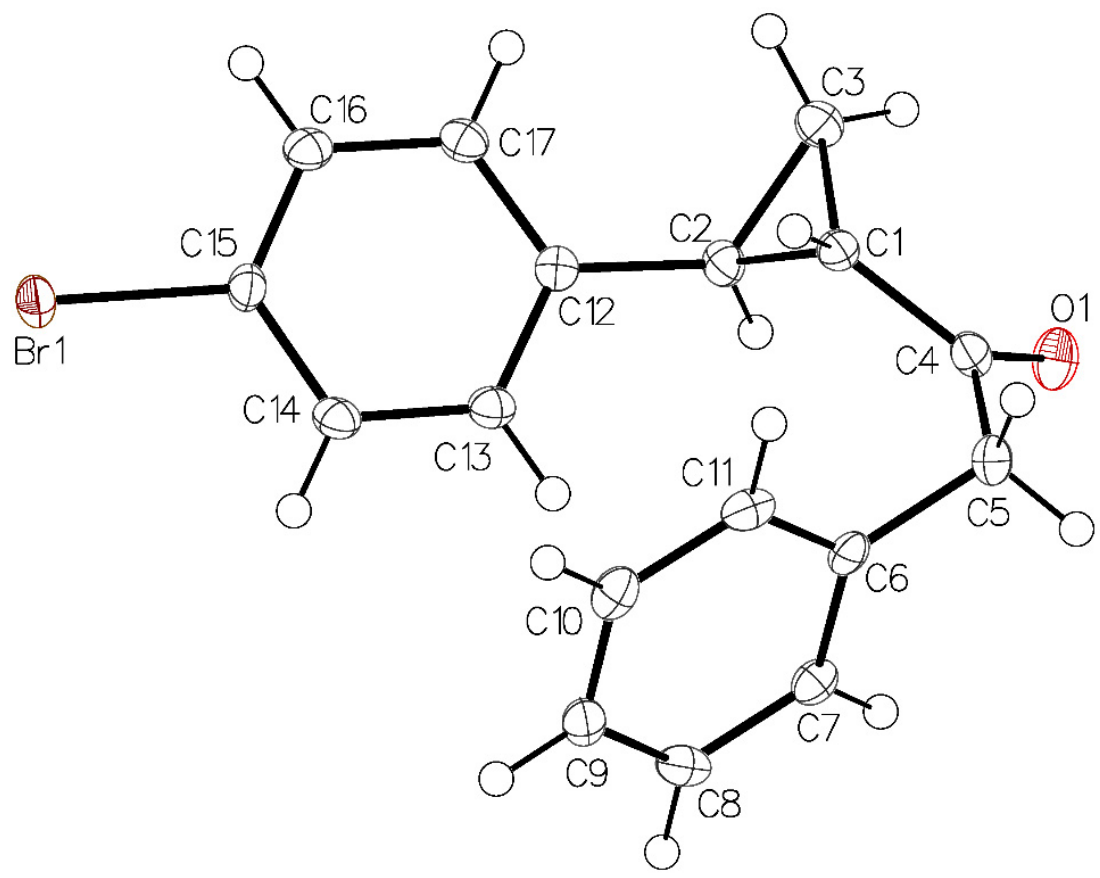


Figure S6. ORTEP of 1-(4-bromo-phenyl)-3-diazo-propan-2-one 8c with ellipsoids drawn at the $50 \%$ probability level. Hydrogen atoms were in the difference Fourier map and refined freely. They are represented here as spheres of arbitrary radius for clarity.

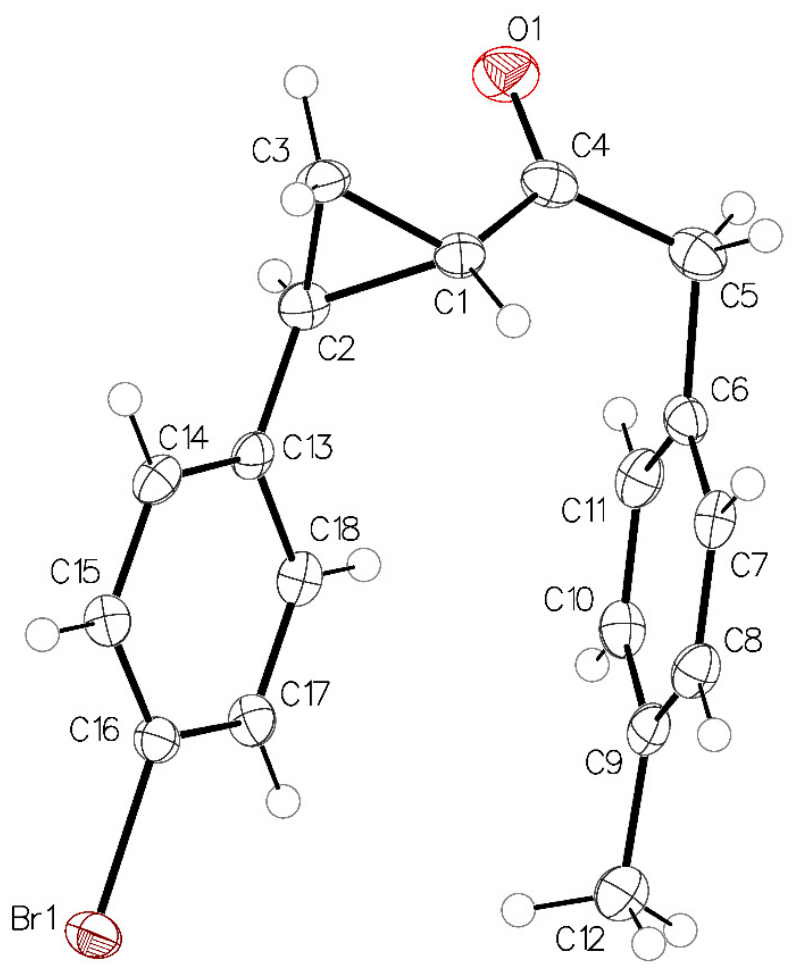




\section{Analytical Methods:}

Chiral SFC Methods. Stereoisomer resolution for compounds 3, 5a-5k, 7a-7i, 8a-8c and 9-18 was performed by Supercritical Fluid Chromatography (SFC) analysis, using a JASCO Analytical and Semi-Preparative SFC instrument equipped with a column oven $\left(35^{\circ} \mathrm{C}\right)$, photodiode array detector, a backpressure regulator $(\sim 14.0 \mathrm{MPa})$, a carbon dioxide pump and a sample injection volume of $3 \mu \mathrm{L}$. Daicel Chiralpak IA, IB, IC or IF column $(0.46 \mathrm{~cm} \mathrm{ID} \times 25 \mathrm{~cm} \mathrm{~L})$ were used for separation of enantiomers. All samples were eluted using an isocratic solvent system with the indicated modifier (see table below) in liquid $\mathrm{CO}_{2}$ at an elution rate of $4 \mathrm{~mL} / \mathrm{min}$ and detected at $\lambda$ $=220 \mathrm{~nm}$. Total run time was $10.2 \mathrm{~min}$.

\begin{tabular}{|c|c|c|c|c|c|c|}
\hline Product & Column & $\begin{array}{c}\text { Modifier } \\
\text { Solvent }\end{array}$ & $\begin{array}{l}\mathrm{t}_{\mathrm{R}} \text { for }(1 S, 2 S) \\
\text { isomer }(\mathrm{min})\end{array}$ & $\begin{array}{l}t_{R} \text { for }(1 R, 2 R) \\
\text { isomer }(\mathrm{min})\end{array}$ & $\begin{array}{c}t_{R} \text { for cis } 1 \\
\text { isomer }(\mathrm{min})\end{array}$ & $\begin{array}{c}t_{R} \text { for cis2 } \\
\text { isomer (min) }\end{array}$ \\
\hline 3 & IF & $5 \%$ IPA & 3.84 & 4.28 & $5.60^{a}$ & $5.60^{a}$ \\
\hline $5 \mathbf{a}$ & IF & $7 \%$ IPA & 4.08 & 4.48 & 5.36 & 5.93 \\
\hline $5 b$ & IF & $10 \%$ IPA & 3.19 & 3.53 & 4.69 & 5.09 \\
\hline $5 c$ & IF & $7 \%$ IPA & 3.45 & 3.83 & 4.95 & 5.11 \\
\hline $5 d$ & IB & $5 \%$ IPA & 4.32 & 4.55 & 4.83 & 5.13 \\
\hline $5 e$ & IF & $5 \%$ IPA & 2.89 & 3.08 & 3.39 & 3.69 \\
\hline $5 f$ & IB & $3 \%$ IPA & 4.73 & 5.11 & 5.64 & 6.04 \\
\hline $5 g$ & IB & $5 \%$ IPA & 3.32 & 3.63 & 4.87 & 5.41 \\
\hline $5 h$ & IB & $5 \%$ IPA & 2.64 & 3.09 & 3.32 & 3.64 \\
\hline $5 \mathbf{i}$ & IF & $5 \%$ IPA & 1.83 & 1.95 & 4.40 & 5.83 \\
\hline $5 \mathbf{j}$ & IB & $5 \%$ IPA & 3.24 & 3.60 & $-b$ & $-b$ \\
\hline $5 k$ & IB & $5 \%$ IPA & 3.59 & 3.92 & 2.91 & 3.15 \\
\hline $7 \mathbf{a}$ & IF & $8 \%$ IPA & 4.39 & 4.91 & 5.67 & 6.08 \\
\hline $7 \mathbf{b}$ & IF & $8 \%$ IPA & 3.51 & 3.89 & $-b$ & $-b$ \\
\hline $7 c$ & IF & $8 \%$ IPA & 4.32 & 4.78 & 5.51 & 5.72 \\
\hline $7 d$ & IF & $2 \%$ IPA & 4.68 & 5.13 & 5.75 & 6.04 \\
\hline $7 e$ & IB & $5 \%$ IPA & 3.28 & 3.59 & 4.00 & 4.28 \\
\hline $7 f$ & IF & $8 \%$ IPA & 4.17 & 4.63 & $5.93^{a}$ & $5.93^{a}$ \\
\hline $7 g$ & IF & $7 \%$ IPA & 4.20 & 4.61 & 5.58 & 5.76 \\
\hline $7 \mathrm{~h}$ & IF & $7 \%$ IPA & 4.87 & 5.35 & 6.49 & 6.73 \\
\hline $7 \mathbf{i}$ & IB & $2 \%$ IPA & 3.99 & 4.57 & $-b$ & $-b$ \\
\hline $8 \mathbf{a}$ & IF & $20 \%$ IPA & 180 & 1.92 & $2.23^{a}$ & $2.23^{a}$ \\
\hline $8 \mathbf{a}$ & IF & $20 \%$ IPA & 2.05 & 2.21 & 2.69 & 2.83 \\
\hline $8 b$ & IF & $20 \%$ IPA & 2.25 & 2.40 & 2.64 & 2.76 \\
\hline
\end{tabular}




\begin{tabular}{ccccccc}
\hline Product & Column & $\begin{array}{c}\text { Modifier } \\
\text { Solvent }\end{array}$ & $\begin{array}{c}\mathbf{t}_{\mathbf{R}} \text { for } \mathbf{1 s t} \\
\text { isomer (min) }\end{array}$ & $\begin{array}{c}\mathbf{t}_{\mathbf{R}} \text { for 2nd } \\
\text { isomer (min) }\end{array}$ & $\begin{array}{c}\mathbf{t}_{\mathbf{R}} \text { for 3rd } \\
\text { isomer }(\mathbf{m i n})\end{array}$ & $\begin{array}{c}\mathbf{t}_{\mathbf{R}} \text { for 4th } \\
\text { isomer } \\
(\mathbf{m i n})\end{array}$ \\
\hline $\mathbf{9}$ & IB & $10 \%$ IPA & 3.51 & 3.85 & 4.32 & 4.84 \\
$\mathbf{1 0}$ & IB & $15 \%$ IPA & 1.55 & 1.64 & 1.73 & 1.80 \\
$\mathbf{1 1}$ & IB & $10 \%$ IPA & 3.57 & 3.91 & 4.37 & 4.91 \\
$\mathbf{1 2}$ & IA & $20 \%$ IPA & 4.11 & 4.73 & N/A & N/A \\
$\mathbf{1 3}$ & IB & $5 \%$ IPA & 2.75 & 2.96 & N/A & N/A \\
$\mathbf{1 4}$ & IA & 20\% IPA & 3.51 & 3.97 & 4.84 & 5.45 \\
$\mathbf{1 5}$ & IF & $5 \%$ IPA & 1.97 & 2.23 & 2.51 & 2.71 \\
$\mathbf{1 6}$ & IB & $15 \%$ IPA & 1.55 & 1.64 & 1.73 & 1.80 \\
$\mathbf{1 7}$ & IB & 3\% IPA & 3.04 & 3.21 & 3.48 & 3.63 \\
$\mathbf{1 8}$ & IA & 5\% IPA & 3.99 & 4.76 & N/A & N/A \\
\hline
\end{tabular}

Chiral GC Methods. Gas chromatography (GC) analysis were carried out using a Shimadzu GC2010 gas chromatograph equipped with a FID detector, and a Cyclosil-B column $(30 \mathrm{~m}$ x $0.25 \mathrm{~mm}$ x $0.25 \mu \mathrm{m}$ film). The following GC methods were used for TON analysis for $\mathbf{3}, \mathbf{5 a - 5} \mathbf{d}, \mathbf{7 k - 7 n}$ and stereoisomer separation (\% de analysis) for $7 \mathbf{k}-\mathbf{7 n}, 1 \mu \mathrm{L}$ injection, injector temp.: $200{ }^{\circ} \mathrm{C}$, detector temp: $300{ }^{\circ} \mathrm{C}$. Gradient for method A: column temperature set at $140{ }^{\circ} \mathrm{C}$ for $3 \mathrm{~min}$, then to $165^{\circ} \mathrm{C}$ at $1.8^{\circ} \mathrm{C} / \mathrm{min}$, then to $245^{\circ} \mathrm{C}$ at $25^{\circ} \mathrm{C} / \mathrm{min}, 245^{\circ} \mathrm{C}$ hold for $8 \mathrm{~min}$. Total run time was $30.3 \mathrm{~min}$. Gradient for method B: column temperature set at $130{ }^{\circ} \mathrm{C}$ for $2 \mathrm{~min}$, then to $180{ }^{\circ} \mathrm{C}$ at $0.8^{\circ} \mathrm{C} / \mathrm{min}$, then to $245^{\circ} \mathrm{C}$ at $25^{\circ} \mathrm{C} / \mathrm{min}, 245^{\circ} \mathrm{C}$ hold for $13 \mathrm{~min}$. Total run time was $92.6 \mathrm{~min}$.

\begin{tabular}{cccccc}
\hline Product & Method & $\begin{array}{c}\mathbf{t}_{\mathbf{R}} \text { for }(\mathbf{1 S}, \mathbf{2 S}) \\
\mathbf{i s o m e r}(\mathbf{m i n})\end{array}$ & $\begin{array}{c}\mathbf{t}_{\mathbf{R}} \text { for }(\mathbf{1 R , 2 R}) \\
\mathbf{i s o m e r}(\mathbf{m i n})\end{array}$ & $\begin{array}{c}\mathbf{t}_{\mathbf{R}} \text { for cis1 } \\
\text { isomer }(\mathbf{m i n})\end{array}$ & $\begin{array}{c}\mathbf{t}_{\mathbf{R}} \text { for cis2 } \\
\text { isomer (min) }\end{array}$ \\
\hline $\mathbf{3}$ & $\mathrm{A}$ & 23.84 & - & - & - \\
$\mathbf{5 a}$ & $\mathrm{A}$ & 24.86 & - & - & - \\
$\mathbf{5 b}$ & $\mathrm{A}$ & 27.51 & - & - & - \\
$\mathbf{5 c}$ & $\mathrm{A}$ & 26.53 & - & - & - \\
$\mathbf{5 d}$ & $\mathrm{A}$ & 28.51 & - & - & - \\
$\mathbf{7 k}$ & $\mathrm{A}$ & 23.71 & - & - & - \\
$\mathbf{7 l}$ & $\mathrm{A}$ & 20.07 & - & - & - \\
$\mathbf{7 m}$ & $\mathrm{A}$ & 20.32 & - & - & - \\
$\mathbf{7 n}$ & $\mathrm{A}$ & 15.20 & - & - & - \\
$\mathbf{7 k}$ & $\mathrm{B}$ & $70.77^{a}$ & $70.77^{a}$ & 63.29 & 64.04 \\
$\mathbf{7 l}$ & $\mathrm{B}$ & $35.37^{a}$ & $35.37^{a}$ & 30.09 & 30.56 \\
$\mathbf{7 m}$ & $\mathrm{B}$ & 34.10 & 33.87 & 30.55 & 30.99 \\
$\mathbf{7 n}$ & $\mathrm{B}$ & $24.25^{a}$ & $24.25^{a}$ & 20.95 & 21.35 \\
\hline
\end{tabular}

${ }^{a}$ trans enantiomers not resolved 
Chiral HPLC Methods. Stereoisomer resolution for compounds 7k-7n was performed by Higher Performance Liquid Chromatography (HPLC) analysis, using a Prominence-I LC-2030 Plus, equipped with column oven $\left(25^{\circ} \mathrm{C}\right)$, UV/PDA detector and a sample injection volume of $5 \mu \mathrm{L}$. Daicel Chiralpak AD-H column $(0.46 \mathrm{~cm}$ ID $\times 15 \mathrm{~cm} \mathrm{~L})$ was used for separation of enantiomers. All samples were eluted using an 5\% isopropyl alcohol and 95\% hexanes at an elution rate of 1 $\mathrm{mL} / \mathrm{min}$ and detected at $254 \mathrm{~nm}$. Total run time was 6 min.

\begin{tabular}{ccc}
\hline Product & $\begin{array}{c}\mathbf{t}_{\mathbf{R}} \text { for }(\mathbf{1 S , 2 S )} \\
\text { isomer }(\mathbf{m i n})\end{array}$ & $\begin{array}{c}\mathbf{t}_{\mathbf{R}} \text { for }(\mathbf{1 R , 2 R}) \\
\text { isomer }(\mathbf{m i n})\end{array}$ \\
\hline $\mathbf{7 k}$ & 2.85 & 3.13 \\
$\mathbf{7 l}$ & 2.64 & 2.80 \\
$\mathbf{7 m}$ & 3.05 & 3.45 \\
$\mathbf{7 n}$ & 2.65 & 2.86 \\
\hline
\end{tabular}




\section{Compound Characterization Data}

1-Diazo-3-phenyl-2-propanone (2)<smiles>N#CC(=O)Cc1ccccc1</smiles>

Following the standard procedure A, diazoketone 2a was isolated as yellow oil, $79.1 \mathrm{mg}, 82 \%$ yield over two steps. $\mathbf{R}_{\mathbf{f}}: 0.3$ in $20 \%$ EtOAc in hexane. ${ }^{1} \mathbf{H} \mathbf{N M R}\left(500 \mathrm{MHz}, \mathrm{CDCl}_{3}\right): \delta 7.39-7.32$ (t, 2H, $J=7.1 \mathrm{~Hz}), 7.29(\mathrm{~d}, J=7.0 \mathrm{~Hz}, 1 \mathrm{H}), 7.24(\mathrm{t}, J=7.1 \mathrm{~Hz}, 2 \mathrm{H}), 5.12(\mathrm{~s}, 1 \mathrm{H}), 3.62(\mathrm{~s}, 2 \mathrm{H})$; ${ }^{13} \mathbf{C ~ N M R}\left(126 \mathrm{MHz}, \mathrm{CDCl}_{3}\right): \delta 206.8,130.2,129.7,128.1,55.7,48.9$. Quaternary carbon atom was not detectable.

\section{1-(4-Chloro-phenyl)-3-diazo-propan-2-one (6a)}<smiles>N#CC(=O)Cc1ccc(Cl)cc1</smiles>

Following the standard procedure A, diazoketone $\mathbf{2 b}$ was isolated as yellow solid, $90.3 \mathrm{mg}, 77 \%$ yield over two steps. ${ }^{1} \mathbf{H}$ NMR $\left(500 \mathrm{MHz}, \mathrm{CDCl}_{3}\right): \delta 7.31$ (d, $\left.J=8.3 \mathrm{~Hz}, 2 \mathrm{H}\right), 7.17(\mathrm{~d}, J=8.3 \mathrm{~Hz}$, 2H), 5.14 (s, 1H), 3.58 (s, 2H); ${ }^{13} \mathbf{C}$ NMR (126 MHz, $\left.\mathrm{CDCl}_{3}\right): \delta$ 192.7, 148.8 134.1, 131.5, 129.8, $55.7,47.9$.

\section{1-(4-Bromo-phenyl)-3-diazo-propan-2-one (6b)}<smiles>N#CCC(=O)Cc1ccc(Br)cc1</smiles>

Following the standard procedure A, diazoketone $\mathbf{6 b}$ was isolated as yellow solid, $76.2 \mathrm{mg}, 53 \%$ yield over two steps. ${ }^{1} \mathbf{H}$ NMR (500 MHz, $\left.\mathrm{CDCl}_{3}\right): \delta 7.46(\mathrm{~d}, J=8.3 \mathrm{~Hz}, 2 \mathrm{H}), 7.12(\mathrm{~d}, J=8.3 \mathrm{~Hz}$, 2H), 5.14 (s, 1H), 3.56 (s, 2H); ${ }^{13}$ C NMR (126 MHz, CDCl 3 ): $\delta$ 132.7, 131.8, 122.1, 55.7, 47.9. Carbonyl carbon atom and one of the quaternary carbon atoms were not detectable.

\section{1-(3-Bromo-phenyl)-3-diazo-propan-2-one (6c)}<smiles>N=CC(=O)Cc1cccc(Br)c1</smiles>

Following the standard procedure A, diazoketone 6c was isolated as yellow oil, $95.1 \mathrm{mg}, 66 \%$ yield over two steps. ${ }^{1} \mathbf{H}$ NMR $\left(500 \mathrm{MHz}, \mathrm{CDCl}_{3}\right): \delta 7.41(\mathrm{~d}, J=14.9 \mathrm{~Hz}, 1 \mathrm{H}), 7.40(\mathrm{~s}, 1 \mathrm{H}), 7.20$ 
(dd, $J=14.9,14.6 \mathrm{~Hz}, 1 \mathrm{H}), 7.19$ (d, $J=14.6 \mathrm{~Hz}, 1 \mathrm{H}), 5.16(\mathrm{~s}, 1 \mathrm{H}), 3.57(\mathrm{~s}, 2 \mathrm{H}) ;{ }^{13} \mathrm{C}$ NMR $(126$ $\left.\mathrm{MHz}, \mathrm{CDCl}_{3}\right): \delta$ 192.4, 137.4, 133.1, 131.2, 131.1, 128.8, 123.6, 55.9, 48.1.

\section{1-(2-Bromo-phenyl)-3-diazo-propan-2-one (6d)}<smiles>N#CC(=O)Cc1ccccc1Br</smiles>

Following the standard procedure A, diazoketone 6d was isolated as yellow oil, $33.3 \mathrm{mg}, 23 \%$ yield over two steps. ${ }^{1} \mathbf{H}$ NMR $\left(500 \mathrm{MHz}, \mathrm{CDCl}_{3}\right): \delta 7.58(\mathrm{~d}, J=8.0 \mathrm{~Hz}, 1 \mathrm{H}), 7.33-7.29(\mathrm{~m}, 2 \mathrm{H})$, $7.17(\mathrm{~d}, J=8.4 \mathrm{~Hz}, 1 \mathrm{H}), 5.17(\mathrm{~s}, 1 \mathrm{H}), 3.78(\mathrm{~s}, 2 \mathrm{H}) ;{ }^{13} \mathbf{C} \mathbf{N M R}\left(126 \mathrm{MHz}, \mathrm{CDCl}_{3}\right): \delta 192.1,135.4$, $133.8,132.5,129.9,128.6,125.8,55.8,48.7$.

\section{1-Diazo-3-(4-methylphenyl)-2-propanone (6e)}<smiles>Cc1ccc(CC(=O)C=N)cc1</smiles>

Following the standard procedure A, diazoketone 6e was isolated as yellow oil, $56.1 \mathrm{mg}, 54 \%$ yield over two steps. $\mathbf{R}_{\mathbf{f}}: 0.3$ in $20 \%$ EtOAc in hexane. ${ }^{1} \mathbf{H}$ NMR $\left(500 \mathrm{MHz}, \mathrm{CDCl}_{3}\right): \delta 7.15(\mathrm{~d}, J$ $=8.0 \mathrm{~Hz}, 2 \mathrm{H}), 7.12(\mathrm{~d}, J=8.0 \mathrm{~Hz}, 2 \mathrm{H}), 5.11(\mathrm{~s}, 1 \mathrm{H}), 3.57(\mathrm{~s}, 2 \mathrm{H}), 2.34(\mathrm{~s}, 3 \mathrm{H}) ;{ }^{13} \mathbf{C}$ NMR $(126$ $\left.\mathrm{MHz}, \mathrm{CDCl}_{3}\right): \delta 137.7,130.3,130.0,55.4,48.5,21.8$. Carbonyl carbon atom and one of the quaternary carbon atoms were not detectable.

\section{1-Diazo-3-(4-methoxyphenyl)-2-propanone (6f)}<smiles>COc1ccc(CC(=O)C=[W])cc1</smiles>

Following the standard procedure A, diazoketone $\mathbf{6 f}$ was isolated as yellow oil, $40.2 \mathrm{mg}, 35 \%$ yield over two steps. $\mathbf{R}_{\mathbf{f}}: 0.2$ in $20 \%$ EtOAc in hexane. ${ }^{1} \mathbf{H}$ NMR (500 MHz, $\left.\mathrm{CDCl}_{3}\right): \delta 7.15$ (d, $J=8.6$ $\mathrm{Hz}, 2 \mathrm{H}), 6.87$ (d, $J=8.6 \mathrm{~Hz}, 2 \mathrm{H}), 5.11(\mathrm{~s}, 1 \mathrm{H}), 3.80$ (s, 3H), 3.55 (s, 2H); ${ }^{13} \mathbf{C}$ NMR (126 MHz, $\left.\mathrm{CDCl}_{3}\right): \delta 159.6,131.2,115.1,56.0,48.0$. Carbonyl carbon atom and quaternary carbon atoms were not detectable.

\section{1-Diazo-3-(3-methoxyphenyl)-2-propanone (6g)}


$\mathrm{MeO}$<smiles>Cc1cccc(CC(=O)C=[W])c1</smiles>

Following the standard procedure A, diazoketone $\mathbf{6 g}$ was isolated as yellow oil, $62.8 \mathrm{mg}, 54 \%$ yield over two steps. ${ }^{1} \mathbf{H}$ NMR $\left(500 \mathrm{MHz}, \mathrm{CDCl}_{3}\right): \delta 7.29-7.27(\mathrm{~m}, 1 \mathrm{H}), 6.87-6.82(\mathrm{~m}, 2 \mathrm{H})$, $6.80(\mathrm{~s}, 1 \mathrm{H}), 5.16(\mathrm{~s}, 1 \mathrm{H}), 3.83(\mathrm{~s}, 3 \mathrm{H}), 3.61(\mathrm{~s}, 2 \mathrm{H}) ;{ }^{13} \mathbf{C ~ N M R}\left(126 \mathrm{MHz}, \mathrm{CDCl}_{3}\right): \delta$ 160.7, 130.6, $122.5,115.7,113.5,56.0,49.0,30.5$. Quaternary carbon atoms were not detectable.

1-Diazo-3-(3,4-(methylenedioxy)phenyl)-2-propanone (6h)<smiles>N#CC(=O)Cc1ccc2c(c1)OCO2</smiles>

Following the standard procedure $\mathbf{A}$, diazoketone $\mathbf{6 h}$ was isolated as yellow oil, $37.4 \mathrm{mg}, 30 \%$ yield over two steps. ${ }^{1} \mathbf{H}$ NMR $\left(500 \mathrm{MHz}, \mathrm{CDCl}_{3}\right): \delta 6.77(\mathrm{~d}, J=7.9 \mathrm{~Hz}, 1 \mathrm{H}), 6.72(\mathrm{~s}, 1 \mathrm{H}), 6.67$ $(\mathrm{d}, J=7.9 \mathrm{~Hz}, 1 \mathrm{H}), 5.95(\mathrm{~s}, 2 \mathrm{H}), 5.15(\mathrm{~s}, 1 \mathrm{H}), 3.51(\mathrm{~s}, 2 \mathrm{H}) ;{ }^{13} \mathbf{C} \mathbf{N M R}\left(126 \mathrm{MHz}, \mathrm{CDCl}_{3}\right): \delta 148.8$, $147.6,123.3,110.4,109.3,101.9,55.5,48.5$. Carbonyl carbon atom and one of the quaternary carbon atoms were not detectable.

\section{(S)-1-Diazo-3-phenylbutan-2-one (6i)}<smiles>CC(C(=O)C=[W])c1ccccc1</smiles>

Following the standard procedure A, diazoketone 6i was isolated as yellow oil, $72.1 \mathrm{mg}, 71 \%$ yield over two steps. Rf: 0.3 in 20\% EtOAc in hexane. ${ }^{1} \mathbf{H}$ NMR $\left(500 \mathrm{MHz}, \mathrm{CDCl}_{3}\right): \delta 7.34$ (dd, $J=7.4$, $7.4 \mathrm{~Hz}, 2 \mathrm{H}), 7.26(\mathrm{~m}, 3 \mathrm{H}), 5.08(\mathrm{~s}, 1 \mathrm{H}), 3.63(\mathrm{q}, J=7.1 \mathrm{~Hz}, 1 \mathrm{H}), 1.48(\mathrm{~d}, J=7.1 \mathrm{~Hz}, 3 \mathrm{H}) ;{ }^{13} \mathrm{C}$ NMR (126 MHz, $\left.\mathrm{CDCl}_{3}\right): \delta 223.3,174.9,170.2,129.7,128.5,128.1,18.5$. Diazo carbon atom was not detectable.

(R)-1-Diazo-3-phenylbutan-2-one (6j)<smiles>CC(C(=O)C=[W])c1ccccc1</smiles> 
Following the standard procedure A, diazoketone $\mathbf{6 j}$ was isolated as yellow oil, $69.1 \mathrm{mg}$, 66\% yield over two steps. NMR data are identical to $6 \mathbf{i}$.

\section{1-cyclohexyl-3-diazopropan-2-one (6k)}<smiles>[N]=CC(=O)CC1CCCCC1</smiles>

Following the standard procedure $\mathbf{A}$, diazoketone $\mathbf{6 k}$ was isolated as yellow oil, $53.2 \mathrm{mg}, 53 \%$ yield over two steps. $\mathbf{R}_{\mathbf{f}}: 0.4$ in $20 \%$ EtOAc in hexane. ${ }^{1} \mathbf{H}$ NMR (500 MHz, $\left.\mathrm{CDCl}_{3}\right): \delta 5.22(\mathrm{~s}$, 1H), $2.17(\mathrm{~s}, 2 \mathrm{H}), 1.79(\mathrm{~m}, 1 \mathrm{H}), 1.68(\mathrm{~m}, 4 \mathrm{H}) 1.26(\mathrm{~m}, 2 \mathrm{H}), 1.13(\mathrm{~m}, 1 \mathrm{H}), 0.94(\mathrm{~m}, 2 \mathrm{H}) ;{ }^{13} \mathrm{C} \mathrm{NMR}$ $\left(126 \mathrm{MHz} \mathrm{CDCl}_{3}\right): \delta 195.6,55.5,49.6,36.1,33.9,26.9,26.8$.

\section{1-cyclopropyl-3-diazopropan-2-one (61)}<smiles>[N]=CC(=O)CC1CC1</smiles>

Following the standard procedure A, diazoketone $6 \mathbf{l}$ was isolated as yellow oil, $42.2 \mathrm{mg}, 56 \%$ yield over two steps. Rf: 0.3 in 20\% EtOAc in hexane. ${ }^{1} \mathbf{H}$ NMR (500 MHz, CDCl 3$): \delta 5.39$ (s, $\left.1 \mathrm{H}\right), 2.20$ $(\mathrm{d}, J=4.1 \mathrm{~Hz}, 2 \mathrm{H}), 0.95(\mathrm{ddd}, J=5.3,5.0,4.1,1 \mathrm{H}), 0.56(\mathrm{ddd}, J=5.3,5.2,5.0 \mathrm{~Hz}, 2 \mathrm{H}), 0.15$ $(\mathrm{ddd}, J=5.2,5.0,5.0 \mathrm{~Hz}, 2 \mathrm{H}) ;{ }^{13} \mathbf{C} \mathbf{N M R}\left(126 \mathrm{MHz}, \mathrm{CDCl}_{3}\right): \delta 54.9,46.8,7.9$, 5.4. Carbonyl carbon was not detectable.

\section{1-diazo-5-methylhexan-2-one (6m)}<smiles>CC(C)CCC(=O)C=[W]</smiles>

Following the standard procedure A, diazoketone $\mathbf{6 m}$ was isolated as yellow oil, $55.1 \mathrm{mg}, 66 \%$ yield over two steps. $\mathbf{R}_{\mathbf{f}}: 0.3$ in $20 \%$ EtOAc in hexane. ${ }^{1} \mathbf{H} \mathbf{~ N M R}\left(500 \mathrm{MHz}, \mathrm{CDCl}_{3}\right): \delta 5.22(\mathrm{~s}$, 1H), $2.34-2.24(\mathrm{~m}, 2 \mathrm{H}), 1.55(\operatorname{septet}(\mathrm{t}), J=7.4,6.4 \mathrm{~Hz} 1 \mathrm{H}), 1.49(\mathrm{dt}, J=7.4,7.2 \mathrm{~Hz}, 2 \mathrm{H}), 0.87$ $(\mathrm{d}, J=6.4 \mathrm{~Hz}, 6 \mathrm{H}) ;{ }^{13} \mathrm{C}$ NMR $\left(126 \mathrm{MHz}, \mathrm{CDCl}_{3}\right): \delta$ 196.2, 54.9, 50.8, 34.7, 28.5, 23.0.

\section{1-diazo-4-methylpentan-2-one (6n)}<smiles>CC(C)CC(=O)C=[W]</smiles> 
Following the standard procedure A, diazoketone $\mathbf{6 n}$ was isolated as yellow oil, $31.8 \mathrm{mg}, 42 \%$ yield over two steps. $\mathbf{R}_{\mathbf{f}}: 0.2$ in $20 \%$ EtOAc in hexane. ${ }^{1} \mathbf{H}$ NMR $\left(500 \mathrm{MHz}, \mathrm{CDCl}_{3}\right): \delta 5.22$ (s, $1 \mathrm{H}), 2.25-1.98(\mathrm{~m}, 3 \mathrm{H}), 0.95(\mathrm{~d}, J=6.5 \mathrm{~Hz}, 6 \mathrm{H}) ;{ }^{13} \mathbf{C} \mathbf{~ N M R}\left(126 \mathrm{MHz}, \mathrm{CDCl}_{3}\right): \delta$ 195.6, 55.4, $50.8,26.8,23.23$.

\section{2-Phenyl-1-((1S, 2S)-2-phenylcyclopropyl)ethan-1-one (3)}

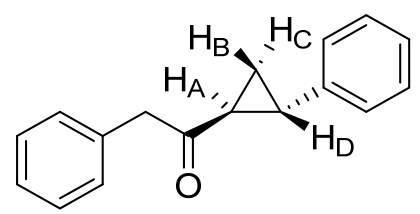

Following the standard procedure B, cyclopropane 3 was isolated as colorless oil, $18.2 \mathrm{mg}, 75 \%$ yield. Rf: 0.3 in 10\% EtOAc in hexane. GC-MS $m / z$ (\% relative intensity): 117 (92.6), 127 (75.1), 145 (100.0), 236 (16.7); ${ }^{1} \mathbf{H}$ NMR (500 MHz, $\left.\mathrm{CDCl}_{3}\right): \delta 7.34$ (t, $\left.J=7.3 \mathrm{~Hz}, 2 \mathrm{H}\right), 7.31-7.17$ (m, $6 \mathrm{H}), 7.01(\mathrm{~d}, J=7.1 \mathrm{~Hz}, 2 \mathrm{H}), 3.88(\mathrm{~s}, 2 \mathrm{H}), 2.52\left(\mathrm{ddd}, J_{\mathrm{A}-\mathrm{C}}=9.1, J_{\mathrm{A}-\mathrm{B}}=6.6, J_{\mathrm{A}-\mathrm{D}}=4.0 \mathrm{~Hz}, \mathrm{H}_{\mathrm{A}}\right)$, $2.23\left(\mathrm{ddd}, J_{\mathrm{D}-\mathrm{B}}=8.1, J_{\mathrm{D}-\mathrm{C}}=5.2, J_{\mathrm{D}-\mathrm{A}}=4.1 \mathrm{~Hz}, \mathrm{HD}\right), 1.69\left(\mathrm{ddd}, J_{\mathrm{C}-\mathrm{A}}=9.2, J_{\mathrm{C}-\mathrm{D}}=5.2, J_{\mathrm{C}-\mathrm{B}}=4.2 \mathrm{~Hz}\right.$, $\mathrm{HC}_{\mathrm{C}}, 1.35$ (ddd, $\left.J_{\mathrm{B}-\mathrm{D}}=8.0, J_{\mathrm{B}-\mathrm{A}}=6.6, J_{\mathrm{B}-\mathrm{C}}=4.2 \mathrm{~Hz}, \mathrm{H}_{\mathrm{B}}\right) ;{ }^{13} \mathbf{C} \mathbf{N M R}\left(126 \mathrm{MHz}, \mathrm{CDCl}_{3}\right): \delta 206.5$, $140.3,134.3,129.7,128.9,128.6,127.2,126.7,126.4,51.2,31.9,29.8,19.2$.

\section{2-Phenyl-1-((1S, 2S)-2-(p-tolyl)cyclopropyl)ethan-1-one (5a)}

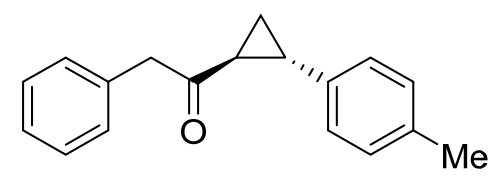

Following the standard procedure B, cyclopropane $\mathbf{5 b}$ was isolated as colorless oil, $19.6 \mathrm{mg}, 78 \%$ yield. GC-MS $m / z$ (\% relative intensity): 115 (38.0), 131 (100.0), 141 (33.1), 159 (67.9), 250 (24.5); ${ }^{1} \mathbf{H}$ NMR (500 MHz, $\left.\mathrm{CDCl}_{3}\right): \delta 7.34(\mathrm{t}, J=7.3 \mathrm{~Hz}, 2 \mathrm{H}), 7.31-7.25(\mathrm{~m}, 1 \mathrm{H}), 7.24$ (d, $J=$ $7.2 \mathrm{~Hz}, 2 \mathrm{H}), 7.07$ (d, $J=7.8 \mathrm{~Hz}, 2 \mathrm{H}), 6.91(\mathrm{~d}, J=7.9 \mathrm{~Hz}, 2 \mathrm{H}), 3.87(\mathrm{~s}, 2 \mathrm{H}), 2.52-2.46(\mathrm{~m}, 1 \mathrm{H})$, 2.32 (s, 3H), $2.22-2.17$ (m, 1H), 1.68 (ddd, $J=9.1,4.7,4.7 \mathrm{~Hz}, 1 \mathrm{H}$ ), 1.33 (ddd, $J=7.8,7.0,4.2$ $\mathrm{Hz}, 1 \mathrm{H}) ;{ }^{13} \mathbf{C}$ NMR $\left(126 \mathrm{MHz}, \mathrm{CDCl}_{3}\right): \delta 207.2,137.9,137.0,135.0,130.3,130.0,129.5,127.8$, $127.0,51.8,32.5,30.3,21.8,19.7$. 


\section{1-((1S, 2S)-2-(4-Methoxyphenyl)cyclopropyl)-2-phenylethan-1-one (5b)}<smiles>COc1ccc([C@@H]2C[C@H]2C(=O)Cc2ccccc2)cc1</smiles>

Following the standard procedure B, cyclopropane $\mathbf{5 b}$ was isolated as colorless oil, $22.1 \mathrm{mg}, 81 \%$ yield. GC-MS $m / z$ (\% relative intensity): 115 (17.9), 132 (9.9), 147 (100.0), 160 (24.2), 175 (24.8), 266 (34.4); ${ }^{1} \mathbf{H}$ NMR (500 MHz, $\left.\mathrm{CDCl}_{3}\right): \delta 7.34$ (t, $\left.J=7.3 \mathrm{~Hz}, 2 \mathrm{H}\right), 7.29$ - 7.26 (m, 1H), 7.23 (d, $J=7.0 \mathrm{~Hz}, 2 \mathrm{H}), 6.92(\mathrm{~d}, J=8.6 \mathrm{~Hz}, 2 \mathrm{H}), 6.79(\mathrm{~d}, J=8.7 \mathrm{~Hz}, 2 \mathrm{H}), 3.86(\mathrm{~s}, 2 \mathrm{H}), 3.79(\mathrm{~s}, 3 \mathrm{H}), 2.47$ $(\mathrm{ddd}, J=9.2,6.7,4.0 \mathrm{~Hz}, 1 \mathrm{H}), 2.18-2.11(\mathrm{~m}, 1 \mathrm{H}), 1.68-1.62(\mathrm{~m}, 1 \mathrm{H}), 1.30$ (ddd, $J=7.9,6.8$, $4.2 \mathrm{~Hz}, 1 \mathrm{H}) ;{ }^{13} \mathbf{C}$ NMR $\left(126 \mathrm{MHz}, \mathrm{CDCl}_{3}\right): \delta 207.2,159.1,135.0,132.9,130.3,129.5,128.2$, $127.7,114.6,56.1,51.8,32.4,30.1,19.5$.

\section{1-((1S, 2S)-2-(4-Chlorophenyl)cyclopropyl)-2-phenylethan-1-one (5c)}<smiles>O=C(Cc1ccccc1)[C@H]1C[C@H]1c1ccc(Cl)cc1</smiles>

Following the standard procedure B, cyclopropane 3d was isolated as white solid, $14.7 \mathrm{mg}, 54 \%$ yield. GC-MS $m / z$ (\% relative intensity): 115 (47.6), 144 (100.0), 179 (50.2), 270 (25.8); ${ }^{1}$ H NMR $\left(500 \mathrm{MHz}, \mathrm{CDCl}_{3}\right): \delta 7.33(\mathrm{t}, J=7.3 \mathrm{~Hz}, 2 \mathrm{H}), 7.30-7.25(\mathrm{~m}, 2 \mathrm{H}), 7.24-7.17(\mathrm{~m}, 3 \mathrm{H}), 6.89(\mathrm{~d}$, $J=8.4 \mathrm{~Hz}, 2 \mathrm{H}), 3.86$ (s, 2H), 2.45 (ddd, $J=9.8,6.5,4.1 \mathrm{~Hz} 1 \mathrm{H}), 2.20-2.10$ (m, 1H), 1.67 (ddd, $J=9.3,4.9,4.8 \mathrm{~Hz}, 1 \mathrm{H}), 1.35-1.27(\mathrm{~m}, 1 \mathrm{H}) ;{ }^{13} \mathbf{C ~ N M R}\left(126 \mathrm{MHz}, \mathrm{CDCl}_{3}\right): \delta 206.4,194.3,139.4$, $134.8,133.0,130.2,129.5,129.3,128.4,127.8,51.8,32.3,29.7,19.6$.

\section{1-((1S, 2S)-2-(4-Bromophenyl)cyclopropyl)-2-phenylethan-1-one (5d)}<smiles>O=C(Cc1ccccc1)C1C[C@H]1c1ccc(Br)cc1</smiles>

Following the standard procedure B, cyclopropane 5d was isolated as white solid, $15.2 \mathrm{mg}, 47 \%$ yield. GC-MS $m / z$ (\% relative intensity): 116 (35.7), 144 (100.0), 314 (8.8), 316 (8.4); ${ }^{1}$ H NMR $\left(500 \mathrm{MHz}, \mathrm{CDCl}_{3}\right): \delta 7.34(\mathrm{~m}, 3 \mathrm{H}), 7.27(\mathrm{~d}, J=12.5 \mathrm{~Hz}, 2 \mathrm{H}), 7.22(\mathrm{~d}, J=7.0 \mathrm{~Hz}, 2 \mathrm{H}), 6.84(\mathrm{~d}, J$ $=7.8 \mathrm{~Hz}, 2 \mathrm{H}), 3.86(\mathrm{~s}, 2 \mathrm{H}), 2.46-2.40(\mathrm{~m}, 1 \mathrm{H}), 2.19-2.13(\mathrm{~m}, 1 \mathrm{H}), 1.71-1.62(\mathrm{~m}, 1 \mathrm{H}), 1.32-$ 
$1.26(\mathrm{~m}, 1 \mathrm{H}) .{ }^{13} \mathrm{C}$ NMR (126 MHz, $\left.\mathrm{CDCl}_{3}\right): \delta 206.8,139.9,134.7,133.1,132.2,130.3,129.6$, $128.8,127.8,51.8,32.3,29.7,19.6$.

1-((1S, 2S)-2-(4-(Trifluoromethyl)phenyl)cyclopropyl)-2-phenylethan-1-one (5e)<smiles>O=C(Cc1ccccc1)[C@@H]1C[C@H]1c1ccc(C(F)(F)F)cc1</smiles>

Following the standard procedure B, cyclopropane 5e was isolated as white solid, $14.3 \mathrm{mg}$, 47\% yield. GC-MS $m / z$ (\% relative intensity): 115 (26.3), 127 (55.5), 144 (21.9), 165 (28.3), 193 (27.5), 213 (100.0), 304 (32.0); ${ }^{1} \mathbf{H}$ NMR (500 MHz, $\mathrm{CDCl}_{3}$ ): $\delta 7.49$ (d, $\left.J=8.1 \mathrm{~Hz}, 2 \mathrm{H}\right), 7.33$ (t, $J=7.2$ $\mathrm{Hz}, 2 \mathrm{H}), 7.30-7.25(\mathrm{~m}, 1 \mathrm{H}), 7.22(\mathrm{~d}, J=7.9 \mathrm{~Hz}, 2 \mathrm{H}), 7.07(\mathrm{~d}, J=8.0 \mathrm{~Hz}, 2 \mathrm{H}), 3.87(\mathrm{~s}, 2 \mathrm{H}), 2.59$ - $2.48(\mathrm{~m}, 1 \mathrm{H}), 2.26-2.20(\mathrm{~m}, 1 \mathrm{H}), 1.72$ (ddd, $J=9.4,4.8,4.7 \mathrm{~Hz}, 1 \mathrm{H}), 1.35$ (ddd, $J=8.0,6.6$, $4.4 \mathrm{~Hz}, 1 \mathrm{H}) ;{ }^{13} \mathbf{C}$ NMR (126 MHz, $\left.\mathrm{CDCl}_{3}\right): \delta 206.6,145.1,134.7,130.2,129.6,127.9,127.3$, 126.1, 126.1, 51.8, 32.5, 29.7, 19.9. $\mathrm{CF}_{3}$ carbon atom was not dectectable.; ${ }^{19} \mathrm{~F}$ NMR $(376 \mathrm{MHz}$, $\left.\mathrm{CDCl}_{3}\right):-62.61(\mathrm{~s})$.

\section{2-Phenyl-1-((1S, 2S)-2-(m-tolyl)cyclopropyl)ethan-1-one (5f)}<smiles>Cc1cccc([C@@H]2C[C@H]2C(=O)Cc2ccccc2)c1</smiles>

Following the standard procedure B, cyclopropane $\mathbf{5 f}$ was isolated as colorless oil, $11.3 \mathrm{mg}, 42 \%$ yield. GC-MS m/z (\% relative intensity): 115 (34.6), 131 (100.0), 141 (31.6), 159 (70.5), 250 (11.0); ${ }^{1} \mathbf{H}$ NMR $\left(500 \mathrm{MHz}, \mathrm{CDCl}_{3}\right): \delta 7.34(\mathrm{t}, J=7.4 \mathrm{~Hz}, 2 \mathrm{H}), 7.30-7.26(\mathrm{~m}, 1 \mathrm{H}), 7.24(\mathrm{~d}, J=$ $7.3 \mathrm{~Hz}, 2 \mathrm{H}), 7.15$ (t, $J=7.5 \mathrm{~Hz}, 1 \mathrm{H}), 7.01(\mathrm{~d}, J=7.5 \mathrm{~Hz}, 1 \mathrm{H}), 6.81(\mathrm{~d}, J=7.9 \mathrm{~Hz}, 2 \mathrm{H}), 3.87$ (s, 2H), $2.51-2.45(\mathrm{~m}, 1 \mathrm{H}), 2.31(\mathrm{~s}, 3 \mathrm{H}), 2.24-2.18(\mathrm{~m}, 1 \mathrm{H}), 1.67(\mathrm{ddd}, J=9.1,4.6,4.2 \mathrm{~Hz}, 1 \mathrm{H})$, $1.34(\mathrm{ddd}, J=7.5,4.6,4.2 \mathrm{~Hz}, 1 \mathrm{H}) ;{ }^{13} \mathbf{C} \mathbf{N M R}\left(126 \mathrm{MHz}, \mathrm{CDCl}_{3}\right): \delta 207.1,140.8,138.8,134.9$, $130.3,129.5,129.1,128.1,127.9,127.8,124.0,51.8,32.4,30.5,22.1,19.7$.

\section{2-Phenyl-1-((1S, 2S)-2-(o-tolyl)cyclopropyl)ethan-1-one (5g)}<smiles>Cc1ccccc1[C@@H]1C[C@H]1C(=O)Cc1ccccc1</smiles> 
Following the standard procedure B, cyclopropane $\mathbf{5 g}$ was isolated as colorless oil, $9.6 \mathrm{mg}$, 40\% yield. GC-MS $m / z$ (\% relative intensity): 115 (34.9), 131 (100.0), 141 (37.1), 159 (76.0), 250 (6.8); ${ }^{1} \mathbf{H}$ NMR (500 MHz, $\left.\mathrm{CDCl}_{3}\right): \delta 7.35(\mathrm{t}, J=7.3 \mathrm{~Hz}, 2 \mathrm{H}), 7.31-7.25(\mathrm{~m}, 3 \mathrm{H}), 7.16-7.12(\mathrm{~m}, 2 \mathrm{H})$, $7.11-7.07(\mathrm{~m}, 1 \mathrm{H}), 6.86(\mathrm{~d}, J=7.3 \mathrm{~Hz}, 1 \mathrm{H}), 3.90(\mathrm{~s}, 2 \mathrm{H}), 2.55$ (ddd, $J=8.7,8.7,4.5 \mathrm{~Hz}, 1 \mathrm{H})$, 2.22 (s, 3H), $2.13(\mathrm{~m}, 1 \mathrm{H}), 1.64$ (ddd, $J=8.9,4.5,4.5 \mathrm{~Hz}, 1 \mathrm{H}), 1.37$ (ddd, $J=7.6,7.6,3.9 \mathrm{~Hz}$, 1H). ${ }^{13}$ C NMR (126 MHz, $\left.\mathrm{CDCl}_{3}\right): \delta 207.6,138.7,138.7,134.9,130.6,130.3,129.6,127.8,127.5$, $126.6,126.5,51.8,30.5,28.6,20.2,18.9$.

\section{1-((1S, 2S)-2-Methyl-2-phenylcyclopropyl)-2-phenylethan-1-one (5h)}

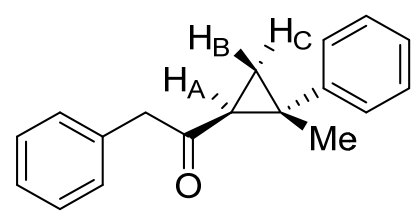

Following the standard procedure $\mathbf{B}$, cyclopropane $\mathbf{5 h}$ was isolated as colorless oil, $10.9 \mathrm{mg}, 21 \%$ yield. GC- MS $m / z$ (\% relative intensity): 103 (45.4), 117 (100.0), 131 (43.8), 159 (95.9), 250 (31.3); ${ }^{1} \mathbf{H}$ NMR (500 MHz, CDCl $): \delta 7.37$ (t, $\left.J=7.2 \mathrm{~Hz}, 2 \mathrm{H}\right), 7.33$ (d, $\left.J=7.2 \mathrm{~Hz}, 2 \mathrm{H}\right), 7.28-$ $7.24(\mathrm{~m}, 2 \mathrm{H}), 7.22-7.13(\mathrm{~m}, 2 \mathrm{H}), 6.89-6.85(\mathrm{~m}, 2 \mathrm{H}), 3.86\left(\mathrm{~d},{ }^{2} J=14.5 \mathrm{~Hz}, 1 \mathrm{H}\right), 3.83\left(\mathrm{~d},{ }^{2} J=\right.$ $14.5 \mathrm{~Hz}, 1 \mathrm{H}), 2.25\left(\mathrm{dd}, J_{\mathrm{A}-\mathrm{C}}=7.7, J_{\mathrm{A}-\mathrm{B}}=6.1, \mathrm{~Hz}, \mathrm{H}_{\mathrm{A}}\right), 1.59\left(\mathrm{dd}, J_{\mathrm{B}-\mathrm{A}}=5.9, J_{\mathrm{B}-\mathrm{C}}=4.5 \mathrm{~Hz}, \mathrm{H}_{\mathrm{B}}\right), 1.39$ $\left(\mathrm{dd}, J_{\mathrm{C}-\mathrm{A}}=7.8, J_{\mathrm{C}-\mathrm{B}}=4.4 \mathrm{~Hz}, \mathrm{H}_{\mathrm{C}}\right), 1.26(\mathrm{~s}, 3 \mathrm{H}) ;{ }^{13} \mathbf{C} \mathbf{N M R}\left(126 \mathrm{MHz}, \mathrm{CDCl}_{3}\right): \delta 206.0,146.6$, 135.1, 130.4, 129.6, 129.1, 128.0, 127.8, 127.2, 53.2, 36.1, 35.4, 22.1, 20.0.

\section{1-((1S, 2S)-2-(Perfluorophenyl)cyclopropyl)-2-phenylethan-1-one (5i)}<smiles>O=C(Cc1ccccc1)C1C[C@H]1c1c(F)c(F)c(F)c(F)c1F</smiles>

Following the standard procedure B, cyclopropane 5i was isolated as colorless oil, $12.8 \mathrm{mg}, 41 \%$ yield. GC- MS m/z (\% relative intensity): 181 (35.7), 187 (29.7), 207 (12.8), 221 (14.1), 235 (100.0), 326 (12.6); ${ }^{1} \mathbf{H}$ NMR (500 MHz, $\left.\mathrm{CDCl}_{3}\right): \delta 7.34$ (t, $\left.J=7.3 \mathrm{~Hz}, 2 \mathrm{H}\right), 7.30-7.25$ (m, 1H), $7.23(\mathrm{~d}, J=7.1 \mathrm{~Hz}, 2 \mathrm{H}), 3.92(\mathrm{~s}, 2 \mathrm{H}), 2.45-2.38(\mathrm{~m}, 2 \mathrm{H}), 1.70-1.65(\mathrm{~m}, 1 \mathrm{H}), 1.47$ (ddd, $J=7.5$, 7.5, $4.5 \mathrm{~Hz}, 1 \mathrm{H}) ;{ }^{13} \mathrm{C}$ NMR $\left(126 \mathrm{MHz}, \mathrm{CDCl}_{3}\right): \delta 206.5,134.4,130.3,129.6,127.9,51.7,28.1$, 
18.2, 17.6. Carbon atoms on perfluorinated aryl ring were not detectable.; ${ }^{19} \mathbf{F}$ NMR (376 MHz, $\left.\mathrm{CDCl}_{3}\right) \delta-80.86(\mathrm{~d}, J=20.9 \mathrm{~Hz}),-93.97(\mathrm{t}, J=20.5 \mathrm{~Hz}),-100.21(\mathrm{~d}, J=20.1 \mathrm{~Hz})$.

\section{1-((1S, 2S)-2-Methyl-2-(thiophen-3-yl)cyclopropyl)-2-phenylethan-1-one (5j)}

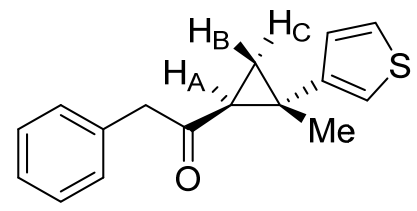

Following the standard procedure B, cyclopropane 5j was isolated as colorless oil, $13.2 \mathrm{mg}$, 52\% yield. GC-MS m/z (\% relative intensity): 111 (15.8), 123 (16.8), 165 (31.2), 223 (71.7), 238 (100.0), 239 (19.0), 256 (12.5); ${ }^{1} \mathbf{H}$ NMR (500 MHz, $\mathrm{CDCl}_{3}$ ): $\delta 7.33$ (dd, $J=7.0,7.0 \mathrm{~Hz}, 2 \mathrm{H}$ ), $7.30-7.24(\mathrm{~m}, 1 \mathrm{H}), 7.24-7.16(\mathrm{~m}, 3 \mathrm{H}), 6.75(\mathrm{~s}, 1 \mathrm{H}), 6.61(\mathrm{~d}, J=4.6 \mathrm{~Hz}, 1 \mathrm{H}), 3.80(\mathrm{~s}, 2 \mathrm{H}), 2.26$ $\left(\mathrm{dd}, J_{\mathrm{A}-\mathrm{C}}=7.1, J_{\mathrm{A}-\mathrm{B}}=5.3 \mathrm{~Hz}, \mathrm{H}_{\mathrm{A}}\right), 1.62\left(\mathrm{dd}, J_{\mathrm{B}-\mathrm{A}}=5.1, J_{\mathrm{B}-\mathrm{C}}=4.7 \mathrm{~Hz}, \mathrm{HB}\right), 1.37\left(\mathrm{dd}, J_{\mathrm{C}-\mathrm{A}}=7.3, J_{\mathrm{C}-}\right.$ $\mathrm{B}=4.5 \mathrm{~Hz}, \mathrm{HC}), 1.25$ (s, 3H); ${ }^{13} \mathbf{C} \mathbf{N M R}\left(126 \mathrm{MHz}, \mathrm{CDCl}_{3}\right): \delta 205.3,147.6,134.9,130.3,129.5$, $127.8,126.5,125.1,120.4,53.1,37.4,30.7,23.1,18.4$.

\section{1-((1S, 2S)-2-Methyl-2-(pyridine-2-yl)cyclopropyl)-2-phenylethan-1-one (5k)}

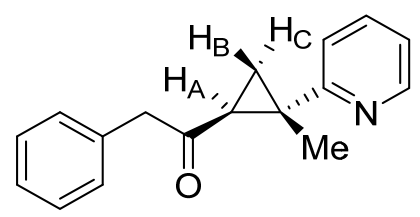

Following the standard procedure B, cyclopropane 5k was isolated as yellow oil, $14.7 \mathrm{mg}, 48 \%$ yield. GC- MS $m / z$ (\% relative intensity): 117 (43.4), 132 (46.7), 160 (100.0), 251 (2.6); ${ }^{1}$ H NMR $\left(500 \mathrm{MHz}, \mathrm{CDCl}_{3}\right): \delta 8.48(\mathrm{~d}, J=4.5 \mathrm{~Hz}, 1 \mathrm{H}), 7.56(\mathrm{ddd}, J=7.8,7.8,1.8 \mathrm{~Hz}, 1 \mathrm{H}), 7.27-7.17$ $(\mathrm{m}, 3 \mathrm{H}), 7.15(\mathrm{~d}, J=6.8 \mathrm{~Hz}, 2 \mathrm{H}), 7.11-7.06(\mathrm{~m}, 2 \mathrm{H}), 3.83\left(\mathrm{~d},{ }^{2} J=15.0 \mathrm{~Hz}, 1 \mathrm{H}\right), 3.79\left(\mathrm{~d},{ }^{2} J=\right.$ $14.5 \mathrm{~Hz}, 1 \mathrm{H}), 2.74\left(\mathrm{dd}, J_{\mathrm{A}-\mathrm{C}}=7.7, J_{\mathrm{A}-\mathrm{B}}=6.7 \mathrm{~Hz}, \mathrm{H}_{\mathrm{A}}\right), 1.76\left(\mathrm{dd}, J_{\mathrm{C}-\mathrm{A}}=7.9, J_{\mathrm{C}-\mathrm{B}}=3.8 \mathrm{~Hz}, \mathrm{HC}_{\mathrm{C}}\right), 1.61$ $\left(\mathrm{dd}, J_{\mathrm{B}-\mathrm{A}}=6.4, J_{\mathrm{B}-\mathrm{C}}=3.8 \mathrm{~Hz}, \mathrm{Hc}\right), 1.31(\mathrm{~s}, 3 \mathrm{H}) .{ }^{13} \mathrm{C}$ NMR $\left(126 \mathrm{MHz}, \mathrm{CDCl}_{3}\right): \delta 205.7,163.0$, $149.7,136.9,134.9,130.3,129.3,127.5,121.8,121.0,52.9,38.1,33.7,23.6,15.9$.

\section{2-(4-Chlorophenyl)-1-((1S, 2S)-2-phenylcyclopropyl)ethan-1-one (7a)}<smiles>O=C(Cc1ccc(Cl)cc1)C1C[C@H]1c1ccccc1</smiles> 
Following the standard procedure B, cyclopropane 7a was isolated as colorless oil, $18.2 \mathrm{mg}, 44 \%$ yield. $\mathbf{R}_{\mathbf{f}}: 0.3$ in $10 \%$ EtOAc in hexane. GC-MS $m / z$ (\% relative intensity): 117 (69.0), 127 (66.7), 145 (100.0), 270 (11.3); ${ }^{1} \mathbf{H}$ NMR (500 MHz, $\mathrm{CDCl}_{3}$ ): $\delta 7.29$ (dd, $\left.J=10.0,7.9 \mathrm{~Hz}, 2 \mathrm{H}\right), 7.26(\mathrm{~d}$, $J=1.8 \mathrm{~Hz}, 2 \mathrm{H}), 7.21(\mathrm{dd}, J=7.3,7.3 \mathrm{~Hz}, 1 \mathrm{H}), 7.15(\mathrm{~d}, J=8.3 \mathrm{~Hz}, 2 \mathrm{H}), 7.02(\mathrm{~d}, J=7.3 \mathrm{~Hz}, 2 \mathrm{H})$, 3.85 (s, 2H), $2.55-2.48$ (m, 1H), $2.22-2.17$ (m, 1H), 1.69 (ddd, $J=9.3,4.8,4.7 \mathrm{~Hz}, 1 \mathrm{H}), 1.38$ (ddd, $J=7.8,6.8,4.6 \mathrm{~Hz}, 1 \mathrm{H}) ;{ }^{13} \mathbf{C}$ NMR $\left(126 \mathrm{MHz}, \mathrm{CDCl}_{3}\right): \delta 206.5,140.7,133.7,133.3,131.6$, $129.6,129.2,127.4,126.9,50.9,32.7,30.5,20.0$.

\section{2-(4-Bromophenyl)-1-((1S, 2S)-2-phenylcyclopropyl)ethan-1-one (7b)}<smiles>O=C(Cc1ccc(Br)cc1)C1C[C@H]1c1ccccc1</smiles>

Following the standard procedure $\mathbf{B}$, cyclopropane $7 \mathbf{b}$ was isolated as colorless oil, $26.8 \mathrm{mg}, 58 \%$ yield. Rf: 0.3 in 10\% EtOAc in hexane. GC-MS $m / z$ (\% relative intensity): 117 (59.0), 127 (46.3), 145 (100.0), 314 (5.9), 316 (5.7); ${ }^{1} \mathbf{H}$ NMR (500 MHz, CDCl ): $\delta 7.45$ (d, $\left.J=8.3 \mathrm{~Hz}, 2 \mathrm{H}\right), 7.28$ (t, $J=7.4 \mathrm{~Hz}, 2 \mathrm{H}), 7.21$ (t, $J=7.3 \mathrm{~Hz}, 1 \mathrm{H}), 7.09$ (d, $J=8.2 \mathrm{~Hz}, 2 \mathrm{H}), 7.02(\mathrm{~d}, J=7.4 \mathrm{~Hz}, 2 \mathrm{H}), 3.83$ (s, $2 \mathrm{H}), 2.52$ (ddd, $J=9.3,6.7,4.0 \mathrm{~Hz}, 1 \mathrm{H}), 2.22-2.16$ (m, $1 \mathrm{H}), 1.69$ (m, $1 \mathrm{H}), 1.38$ (ddd, $J=7.9$, 6.7, $4.2 \mathrm{~Hz}, 1 \mathrm{H}) ;{ }^{13} \mathbf{C} \mathbf{N M R}\left(126 \mathrm{MHz}, \mathrm{CDCl}_{3}\right): \delta 206.3,140.7,133.8,132.6,132.0,129.3,127.4$, $126.9,121.8,50.9,32.8,30.6,20.0$.

\section{2-(3-Bromophenyl)-1-((1S, 2S)-2-phenylcyclopropyl)ethan-1-one (7c)}<smiles>O=C(Cc1cccc(Br)c1)[C@H]1C[C@H]1c1ccccc1</smiles>

Following the standard procedure B, cyclopropane 7c was isolated as colorless oil, $21.9 \mathrm{mg}, 47 \%$ yield. $\mathbf{R}_{\mathbf{f}}: 0.3$ in $10 \%$ EtOAc in hexane. GC-MS $m / z$ (\% relative intensity): 117 (75.2), 127 (51.5), 145 (100.0), 314 (5.0), 316 (4.9); ${ }^{1} \mathbf{H}$ NMR (500 MHz, $\mathrm{CDCl}_{3}$ ): $\delta 7.50$ (d, $\left.J=12.7 \mathrm{~Hz}, 2 \mathrm{H}\right), 7.42$ $-7.35(\mathrm{~m}, 2 \mathrm{H}), 7.31$ (dd, $J=13.7,7.0 \mathrm{~Hz}, 2 \mathrm{H}), 7.25$ (d, $J=7.3 \mathrm{~Hz}, 1 \mathrm{H}), 7.14(\mathrm{~d}, J=7.3 \mathrm{~Hz}, 2 \mathrm{H})$, 3.95 (s, 2H), $2.66-2.60(\mathrm{~m}, 1 \mathrm{H}), 2.35-2.25(\mathrm{~m}, 1 \mathrm{H}), 1.82$ (ddd, $J=8.7,4.4,4.4 \mathrm{~Hz}, 1 \mathrm{H}), 1.53-$ $1.47(\mathrm{~m}, 1 \mathrm{H}) .{ }^{13} \mathbf{C}$ NMR $\left(126 \mathrm{MHz}, \mathrm{CDCl}_{3}\right): \delta 206.2,140.7,137.0,133.3,131.0,130.9,129.3$, 129.0, 127.4, 127.0, 123.5, 51.0, 32.8, 30.7, 19.9. 


\section{2-(2-Bromophenyl)-1-((1S, 2S)-2-phenylcyclopropyl)ethan-1-one (7d)}<smiles>O=C(Cc1ccccc1Br)C1C[C@H]1c1ccccc1</smiles>

Following the standard procedure B, cyclopropane 7d was isolated as colorless oil, $27.3 \mathrm{mg}, 57 \%$ yield. R: 0.3 in 10\% EtOAc in hexane. GC-MS $m / z$ (\% relative intensity): 117 (68.7), 127 (57.1), 145 (100.0), 314 (6.0), 316 (5.9); ${ }^{1} \mathbf{H}$ NMR (500 MHz, $\left.\mathrm{CDCl}_{3}\right): \delta 7.55$ (d, J= $\left.7.9 \mathrm{~Hz}, 2 \mathrm{H}\right), 7.29-$ $7.22(\mathrm{~m}, 3 \mathrm{H}), 7.22-7.16(\mathrm{~m}, 1 \mathrm{H}), 7.13-7.08(\mathrm{~m}, 1 \mathrm{H}), 7.04$ (d, J=7.3 Hz, 2H), 4.02 (s, 2H), 2.58 $-2.52(\mathrm{~m}, 1 \mathrm{H}), 2.27-2.21(\mathrm{~m}, 1 \mathrm{H}), 1.74-1.67(\mathrm{~m}, 7 \mathrm{H}), 1.37(\mathrm{ddd}, J=8.0,6.8,4.2 \mathrm{~Hz}, 1 \mathrm{H}) ;{ }^{13} \mathbf{C}$ NMR (126 MHz, $\left.\mathrm{CDCl}_{3}\right): \delta 205.7,140.9,135.5,133.6,132.5,129.6,129.2,128.4,127.3,127.0$, 125.9, 51.7, 32.8, 30.4, 19.9.

\section{1-((1S, 2S)-2-Phenylcyclopropyl)-2-(p-tolyl)ethan-1-one (7e)}

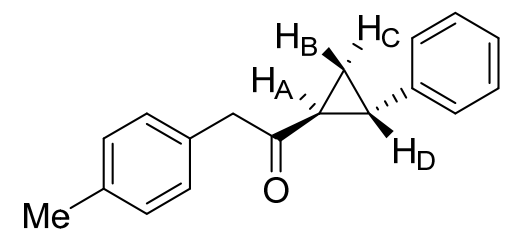

Following the standard procedure B, cyclopropane 7e was isolated as colorless oil, $26.2 \mathrm{mg}, 30 \%$ yield. R: 0.2 in 5\% EtOAc in hexane. GC- MS $m / z$ (\% relative intensity): 105 (97.3), 117 (69.1), 127 (63.0), 145 (100.0), 250 (22.3); ${ }^{1} \mathbf{H}$ NMR (500 MHz, $\left.\mathrm{CDCl}_{3}\right)$ : $\delta 7.27$ - 7.23 (m, 3H), 7.19 (m, $2 \mathrm{H}), 7.12(\mathrm{~m}, 2 \mathrm{H}), 7.00(\mathrm{~d}, J=7.2 \mathrm{~Hz}, 2 \mathrm{H}), 3.82(\mathrm{~s}, 2 \mathrm{H}), 2.50\left(\mathrm{ddd}, J_{\mathrm{A}-\mathrm{C}}=9.2, J_{\mathrm{A}-\mathrm{B}}=6.5, J_{\mathrm{A}-\mathrm{D}}=\right.$ $\left.3.9 \mathrm{~Hz}, \mathrm{H}_{\mathrm{A}}\right), 2.33(\mathrm{~s}, 3 \mathrm{H}), 2.21\left(\mathrm{ddd}, J_{\mathrm{D}-\mathrm{B}}=8.3, J_{\mathrm{D}-\mathrm{C}}=5.1, J_{\mathrm{D}-\mathrm{A}}=4.2 \mathrm{~Hz}, \mathrm{H}_{\mathrm{D}}\right), 1.67\left(\mathrm{ddd}, J_{\mathrm{C}-\mathrm{A}}=\right.$ $\left.9.2, J_{\mathrm{C}-\mathrm{D}}=5.1, J_{\mathrm{C}-\mathrm{B}}=4.2 \mathrm{~Hz}, \mathrm{H}_{\mathrm{C}}\right), 1.33\left(\mathrm{ddd}, J_{\mathrm{B}-\mathrm{D}}=8.1, J_{\mathrm{B}-\mathrm{A}}=6.7, J_{\mathrm{B}-\mathrm{C}}=4.2 \mathrm{~Hz}, \mathrm{H}_{\mathrm{B}}\right) ;{ }^{13} \mathrm{C} \mathrm{NMR}$ $\left(126 \mathrm{MHz}, \mathrm{CDCl}_{3}\right): \delta 210.2,151.8,141.0,137.4,130.2,130.1,129.2,127.2,127.0,51.4,32.4$, $31.7,30.3,19.8$.

\section{2-(4-Methoxyphenyl)-1-((1S, 2S)-2-phenylcyclopropyl)ethan-1-one (7f)}<smiles>COc1ccc(CC(=O)C2C[C@H]2c2ccccc2)cc1</smiles>

Following the standard procedure B, cyclopropane $7 \mathbf{f}$ was isolated as colorless oil, $11.1 \mathrm{mg}, 28 \%$ yield. Rf: 0.2 in 10\% EtOAc in hexane. GC-MS $m / z$ (\% relative intensity): 121 (100.0), 127 (15.4), 
145 (30.7), 266 (20.7); ${ }^{1} \mathbf{H}$ NMR (500 MHz, $\mathrm{CDCl}_{3}$ ): $\delta 7.24$ (d, $\left.J=7.4,2 \mathrm{H}\right), 7.17$ (dd, $J=7.6,7.6$ $\mathrm{Hz}, 1 \mathrm{H}), 7.11(\mathrm{~d}, J=7.7 \mathrm{~Hz}, 2 \mathrm{H}), 6.99$ (d, $J=7.4 \mathrm{~Hz}, 2 \mathrm{H}), 6.84(\mathrm{~d}, J=7.7 \mathrm{~Hz}, 2 \mathrm{H}), 3.78(\mathrm{~s}, 2 \mathrm{H})$, $2.50-2.44(\mathrm{~m}, 1 \mathrm{H}), 2.22-2.16(\mathrm{~m}, 1 \mathrm{H}), 1.68-1.61(\mathrm{~m}, 1 \mathrm{H}), 1.35-1.28(\mathrm{~m}, 1 \mathrm{H}), 1.24(\mathrm{~s}, 3 \mathrm{H})$; ${ }^{13}$ C NMR (126 MHz, $\left.\mathrm{CDCl}_{3}\right): \delta 170.8,167.0,131.2,129.2,127.2,127.0,126.9,114.9,56.0,50.8$, $32.4,30.3,19.8$. Carbonyl carbon atom was not detectable.

\section{2-(3-Methoxyphenyl)-1-((1S, 2S)-2-phenylcyclopropyl)ethan-1-one (7g)}<smiles>COc1cccc(CC(=O)[C@@H]2C[C@H]2c2ccccc2)c1</smiles>

Following the standard procedure $\mathbf{B}$, cyclopropane $\mathbf{7 g}$ was isolated as colorless oil, $30.2 \mathrm{mg}$, $74 \%$ yield. Rf: 0.2 in 10\% EtOAc in hexane. GC-MS $m / z$ (\% relative intensity): 117 (78.0), 127 (78.7), 145 (100.0), 162 (60.1), 175 (29.2), 266 (28.6); ${ }^{1} \mathbf{H}$ NMR (500 MHz, $\left.\mathrm{CDCl}_{3}\right): \delta 7.25$ - 7.20 (m, 3H), 7.17 (dd, $J=7.2,7.2 \mathrm{~Hz}, 1 \mathrm{H}), 6.99$ (d, $J=7.5 \mathrm{~Hz}, 2 \mathrm{H}), 6.79$ (d, $J=7.9 \mathrm{~Hz}, 2 \mathrm{H}), 6.74(\mathrm{~s}, 1 \mathrm{H})$, 3.81 (s, 2H), 3.75 (s, 3H), $2.51-2.45$ (m, 1H), $2.23-2.18$ (m, 1H), 1.67 (ddd, $J=9.2,4.7,4.7$ $\mathrm{Hz}, 1 \mathrm{H}), 1.35-1.30(\mathrm{~m}, 1 \mathrm{H}) ;{ }^{13} \mathbf{C} \mathbf{~ N M R}\left(126 \mathrm{MHz}, \mathrm{CDCl}_{3}\right): \delta 207.0,160.6,140.9,136.3,130.5$, 129.2, 127.3, 127.0, 122.6, 115.8, 113.4, 55.9, 51.8, 32.5, 30.5, 19.8 .

\section{2-(Benzo[1,3]dioxol-5-yl)-1-((1S, 2S)-2-phenylcyclopropyl)ethan-1-one (7h)}

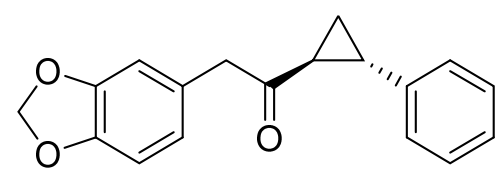

Following the standard procedure B, cyclopropane $7 \mathbf{h}$ was isolated as colorless oil, $14.3 \mathrm{mg}, 34 \%$ yield. $\mathbf{R}_{\mathbf{f}}: 0.2$ in $10 \%$ EtOAc in hexane. GC-MS $m / z$ (\% relative intensity): 117 (24.4), 135 (100.0), 145 (40.6), 280 (39.7); ${ }^{1} \mathbf{H}$ NMR (500 MHz, $\mathrm{CDCl}_{3}$ ): $\delta 7.26$ (dd, $\left.J=7.4,7.4 \mathrm{~Hz}, 2 \mathrm{H}\right), 7.20$ (dd, $J$ $=7.3,7.3 \mathrm{~Hz}, 1 \mathrm{H}), 7.03(\mathrm{~d}, J=7.4 \mathrm{~Hz}, 2 \mathrm{H}), 6.78-6.64(\mathrm{~m}, 3 \mathrm{H}), 5.94\left(\mathrm{~d},{ }^{4} J=0.4 \mathrm{~Hz}, 2 \mathrm{H}\right), 3.77$ (s, $2 \mathrm{H}), 2.53-2.47(\mathrm{~m}, 1 \mathrm{H}), 2.24-2.16(\mathrm{~m}, 1 \mathrm{H}), 1.71-1.64(\mathrm{~m}, 1 \mathrm{H}), 1.38-1.32(\mathrm{~m}, 1 \mathrm{H}),{ }^{13} \mathrm{C}$ NMR (126 MHz, $\left.\mathrm{CDCl}_{3}\right): \delta$ 148.7, 147.4, 140.9, 129.2, 128.5, 127.3, 126.9, 123.4, 110.6, 109.2, 101.8, 51.3, 32.4, 30.4, 19.9. Carbonyl carbon atom was not detectable. 


\section{(S)-2-Phenyl-1-((1S, 2S)-2-phenylcyclopropyl)propan-1-one (7i)}

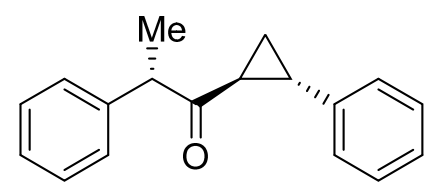

Following the standard procedure B, cyclopropane 7i was isolated as colorless oil, $19.1 \mathrm{mg}, 50 \%$ yield. R: 0.2 in 5\% EtOAc in hexane. GC-MS $m / z$ (\% relative intensity): 105 (79.8), 117 (45.3), 127 (48.2), 145 (100.0), 250 (14.8); ${ }^{1} \mathbf{H}$ NMR (500 MHz, $\left.\mathrm{CDCl}_{3}\right): \delta 7.38$ (dd, $\left.J=7.4 \mathrm{~Hz}, 3 \mathrm{H}\right)$, $7.29-7.23(\mathrm{~m}, 5 \mathrm{H}), 7.03(\mathrm{~d}, J=7.2 \mathrm{~Hz}, 2 \mathrm{H}), 3.95(\mathrm{q}, J=6.9 \mathrm{~Hz}, 1 \mathrm{H}), 2.60-2.49(\mathrm{~m}, 1 \mathrm{H}), 2.20$ $-2.10(\mathrm{~m}, 1 \mathrm{H}), 1.69-1.59(\mathrm{~m}, 1 \mathrm{H}), 1.45(\mathrm{~d}, J=6.9 \mathrm{~Hz}, 3 \mathrm{H}), 1.31-1.23(\mathrm{~m}, 1 \mathrm{H}) ;{ }^{13} \mathbf{C}$ NMR $(126$ $\left.\mathrm{MHz}_{\mathrm{CDCl}}\right): \delta 209.4,141.4,141.2,129.7,129.2,128.9,127.9,127.2,126.9,54.7,32.5,30.4$, $20.1,18.4$.

\section{2-cyclohexyl-1-((1S,2S)-2-phenylcyclopropyl)ethan-1-one (7k)}<smiles>O=C(CC1CCCCC1)[C@H]1C[C@H]1c1ccccc1</smiles>

Following the standard procedure B, cyclopropane $7 \mathbf{k}$ was isolated as colorless oil, $18.3 \mathrm{mg}, 52 \%$ yield. R: 0.2 in 5\% EtOAc in hexane. GC-MS $m / z$ (\% relative intensity): 104 (50.0), 115 (100.0), 127 (25.1), 145 (20.0), 160 (72.9), 242 (1.95) ; ${ }^{1} \mathbf{H}$ NMR (500 MHz, CDCl $)$ : $\delta 7.29$ (dd, J= 7.3, $7.4 \mathrm{~Hz}, 2 \mathrm{H}), 7.21$ (t, $J=7.3 \mathrm{~Hz}, 1 \mathrm{H}), 7.09$ (d, $J=7.4 \mathrm{~Hz}, 2 \mathrm{H}), 2.49$ (m, 1H), 2.45 (d, J=6.9 Hz, 2H), $2.19(\mathrm{~m}, 1 \mathrm{H}), 1.89(\mathrm{~m}, 1 \mathrm{H}), 1.68(\mathrm{~m}, 6 \mathrm{H}), 1.35(\mathrm{ddd}, J=8.0,6.6,4.2 \mathrm{~Hz}, 1 \mathrm{H}), 1.26(\mathrm{~m}, 2 \mathrm{H})$, $1.14(\mathrm{~m}, 1 \mathrm{H}), 1.6(\mathrm{~m}, 2 \mathrm{H}) ;{ }^{13} \mathbf{C}$ NMR (126 MHz, $\left.\mathrm{CDCl}_{3}\right): \delta 209.6,141.3,129.2,127.2,126.8,52.6$, $35.0,34.0,34.0,33.4,29.7,26.9,26.8,19.4$.

\section{2-cyclopropyl-1-((1S,2S)-2-phenylcyclopropyl)ethan-1-one (7l)}

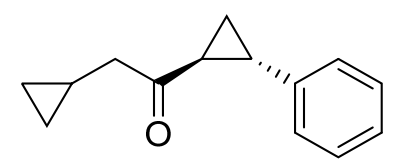

Following the standard procedure B, cyclopropane $7 \mathbf{l}$ was isolated as colorless oil, $10.9 \mathrm{mg}, 28 \%$ yield. R: 0.2 in 5\% EtOAc in hexane. GC-MS $m / z$ (\% relative intensity): 104 (38.8), 115 (100.0), 127 (27.8), 145 (22.4), 160 (59.8), 201 (1.3) ; ${ }^{1} \mathbf{H}$ NMR (500 MHz, $\mathrm{CDCl}_{3}$ ): $\delta 7.26$ (dd, J = 7.4, $7.3 \mathrm{~Hz}, 2 \mathrm{H}), 7.19$ (t, $J=7.3 \mathrm{~Hz}, 1 \mathrm{H}), 7.08(\mathrm{~d}, J=7.4 \mathrm{~Hz}, 2 \mathrm{H}), 2.50(\mathrm{~m}, 1 \mathrm{H}), 2.46(\mathrm{~d}, J=6.9 \mathrm{~Hz}$, 
2H), $2.24(\mathrm{~m}, 1 \mathrm{H}), 1.67(\mathrm{~m}, 1 \mathrm{H}), 1.35(\mathrm{ddd}, J=8.0,6.6,4.1 \mathrm{~Hz}, 1 \mathrm{H}), 1.03(\mathrm{~m}, 1 \mathrm{H}), 0.55(\mathrm{~m}, 2 \mathrm{H})$, 0.13 (m, 2H); ${ }^{13} \mathbf{C}$ NMR (126 MHz, $\left.\mathrm{CDCl}_{3}\right): \delta 209.5,141.3,129.2,127.2,126.8,49.7,32.6,29.8$, 19.6, 7.0, 5.3, 5.2.

\section{4-methyl-1-((1S,2S)-2-phenylcyclopropyl)pentan-1-one (7m)<smiles>CC(C)CCC(=O)[C@@H]1C[C@H]1c1ccccc1</smiles>

Following the standard procedure $\mathbf{B}$, cyclopropane $7 \mathrm{~m}$ was isolated as colorless oil, $16.2 \mathrm{mg}, 36 \%$ yield. Rf: 0.2 in 5\% EtOAc in hexane. GC-MS $m / z$ (\% relative intensity): 115 (100.0), 127 (40.9), 144 (49.4); ${ }^{1} \mathbf{H}$ NMR (500 MHz, $\left.\mathrm{CDCl}_{3}\right): \delta 7.29$ (dd, $\left.J=7.5,7.3 \mathrm{~Hz}, 2 \mathrm{H}\right), 7.21(\mathrm{t}, J=7.5 \mathrm{~Hz}, 1 \mathrm{H})$, 7.10 (d, $J=7.3 \mathrm{~Hz}, 2 \mathrm{H}), 2.60$ (t, $J=9.8 \mathrm{~Hz}, 2 \mathrm{H}), 2.50$ (ddd, $J=9.3,6.5,4.1 \mathrm{~Hz}, 1 \mathrm{H}$ ), 2.20 (ddd, $J=8.2,5.2,4.1 \mathrm{~Hz}, 1 \mathrm{H}), 1.66(\mathrm{ddd}, J=9.3,5.2,4.3 \mathrm{~Hz}, 1 \mathrm{H}), 1,55(\mathrm{~m}, 1 \mathrm{H}), 1.53(\mathrm{~m}, 2 \mathrm{H}), 1.36$ (ddd, $J=8.2,6.5,4.3 \mathrm{~Hz}, 1 \mathrm{H}), 0.90(\mathrm{dd}, J=6.2,1.3 \mathrm{~Hz}, 6 \mathrm{H}) ;{ }^{13} \mathbf{C}$ NMR $\left(126 \mathrm{MHz}, \mathrm{CDCl}_{3}\right): \delta$ $210.1,141.3,129.2,127.2,126.8,42.9,33.5,32.9,29.6,28.5,23.1,19.6$.

\section{4-methyl-1-((1S,2S)-2-phenylcyclopropyl)pentan-1-one (7n)}

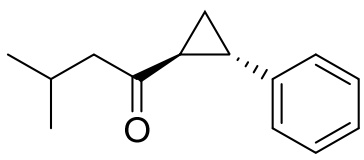

Following the standard procedure B, cyclopropane $\mathbf{7 n}$ was isolated as colorless oil, $12.9 \mathrm{mg}, 21 \%$ yield. Rf: 0.2 in 5\% EtOAc in hexane. GC-MS $m / z$ (\% relative intensity): 104 (25.6), 115 (100.0), 127 (39.2), 145 (22.4) 160 (17.1), 202 (5.9); ${ }^{1} \mathbf{H}$ NMR (500 MHz, CDCl ): $\delta 7.28$ (dd, $J=7.3,7.2$ $\mathrm{Hz}, 2 \mathrm{H}), 7.21$ (t, $J=7.3 \mathrm{~Hz}, 1 \mathrm{H}), 7.09$ (d, $J=7.2 \mathrm{~Hz}, 2 \mathrm{H}), 2.50$ (ddd, $J=9.3,6.6,4.1 \mathrm{~Hz}, 1 \mathrm{H})$, $2.46(\mathrm{~d}, J=7.0 \mathrm{~Hz}, 2 \mathrm{H}), 2.19$ (ddd, $J=8.1,5.2,4.1 \mathrm{~Hz}, 1 \mathrm{H}), 2.18((\mathrm{t})$ septet, $J=7.0,6.7 \mathrm{~Hz}, 1 \mathrm{H})$, 1.67 (ddd, $J=9.3,5.2,4.2 \mathrm{~Hz}, 1 \mathrm{H}$ ), 1.35 (ddd, $J=8.1,6.6,4.2 \mathrm{~Hz}, 1 \mathrm{H}$ ), 0.95 (dd, $J=6.7,2.6 \mathrm{~Hz}$, $6 \mathrm{H}) ;{ }^{13} \mathrm{C}$ NMR $\left(126 \mathrm{MHz}, \mathrm{CDCl}_{3}\right): \delta 209.6,141.2,129.2,127.2,126.8,53.9,33.2,29.6,25.7$, 23.4, 23.4, 19.5. 


\section{2-(p-tolyl)-1-((1S,2S)-2-(p-tolyl)cyclopropyl)ethan-1-one (8a)}

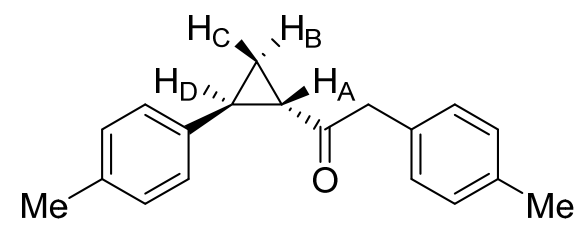

Following the standard procedure B, cyclopropane 8a was isolated as colorless oil, $13.9 \mathrm{mg}, 24 \%$ yield. Rf: 0.2 in 5\% EtOAc in hexane. GC-MS $m / z$ (\% relative intensity): 105 (95.2), 115 (52.6), 117 (91.8), 127 (81.4), 145 (100.0), 250 (22.1); ${ }^{1} \mathbf{H}$ NMR (500 MHz, $\left.\mathrm{CDCl}_{3}\right): \delta 7.14$ (d, $J=8.1$ $\mathrm{Hz}, 2 \mathrm{H}), 7.10(\mathrm{~d}, J=8.1 \mathrm{~Hz}, 2 \mathrm{H}), 7.06$ (d, $J=7.9 \mathrm{~Hz}, 2 \mathrm{H}), 6.90$ (d, J=7.9 Hz, 2H), 3.81 (s, 2H), $2.47\left(\mathrm{ddd}, J_{\mathrm{A}-\mathrm{C}}=9.2, J_{\mathrm{A}-\mathrm{B}}=6.7, J_{\mathrm{A}-\mathrm{D}}=4.2 \mathrm{~Hz}, \mathrm{H}_{\mathrm{A}}\right), 2.34(\mathrm{~s}, 3 \mathrm{H}), 2.31(\mathrm{~s}, 3 \mathrm{H}), 2.18\left(\mathrm{ddd}, J_{\mathrm{D}-\mathrm{B}}=\right.$ $\left.8.1, J_{\mathrm{D}-\mathrm{C}}=5.1, J_{\mathrm{D}-\mathrm{A}}=4.2 \mathrm{~Hz}, \mathrm{H}_{\mathrm{D}}\right), 1.65\left(\mathrm{ddd}, J_{\mathrm{C}-\mathrm{A}}=9.2, J_{\mathrm{C}-\mathrm{D}}=5.1, J_{\mathrm{C}-\mathrm{B}}=4.2 \mathrm{~Hz}, \mathrm{H}_{\mathrm{C}}\right) 1.30(\mathrm{ddd}$, $\left.J_{\mathrm{B}-\mathrm{D}}=8.1, J_{\mathrm{B}-\mathrm{A}}=6.7, J_{\mathrm{B}-\mathrm{C}}=4.2 \mathrm{~Hz}, \mathrm{H}_{\mathrm{B}}\right) .{ }^{13} \mathbf{C} \mathbf{N M R}\left(126 \mathrm{MHz}, \mathrm{CDCl}_{3}\right): \delta 207.4,137.9,137.3$, $136.9,131.8,130.2,130.1,129.8,126.9,51.3,32.4,30.2,21.8,21.7,19.7$.

\section{1-((1S,2S)-2-(4-methoxyphenyl)cyclopropyl)-2-(p-tolyl)ethan-1-one (8b)}<smiles>COc1ccc(CC(=O)[C@@H]2C[C@H]2c2ccc(C)cc2)cc1</smiles>

Following the standard procedure B, cyclopropane $\mathbf{8 b}$ was isolated as colorless oil, $15.8 \mathrm{mg}, 39 \%$ yield. R: 0.2 in 10\% EtOAc in hexane.GC-MS $m / z$ (\% relative intensity): 105 (100), 115 (38.5), 131 (97.7), 141 (41.9), 159 (87.4), 264 (21.1); ${ }^{1} \mathbf{H}$ NMR (500 MHz, CDCl $): \delta 7.14$ (d, $J=8.1 \mathrm{~Hz}$, 2H), $7.11(\mathrm{~d}, J=8.1 \mathrm{~Hz}, 2 \mathrm{H}), 6.92(\mathrm{~d}, J=8.6 \mathrm{~Hz}, 2 \mathrm{H}), 6.79(\mathrm{~d}, J=8.6 \mathrm{~Hz}, 2 \mathrm{H}), 3.82(\mathrm{~s}, 2 \mathrm{H}), 3.78$ (s, 3H), $2.49-2.43(\mathrm{~m}, 1 \mathrm{H}), 2.34(\mathrm{~s}, 3 \mathrm{H}), 2.17-2.11(\mathrm{~m}, 1 \mathrm{H}), 1.67-1.61(\mathrm{~m}, 1 \mathrm{H}), 1.28$ (ddd, $J$ $=7.9,6.7,4.1 \mathrm{~Hz}, 1 \mathrm{H}) ;{ }^{13} \mathbf{C}$ NMR $\left(126 \mathrm{MHz}, \mathrm{CDCl}_{3}\right): \delta 207.4,159.1,137.3,133.0,131.9,130.2$, $130.1,128.2,114.6,56.0,51.4,32.3,29.9,21.8,19.5$.

\section{1-((1S,2S)-2-(4-methoxyphenyl)cyclopropyl)-2-(p-tolyl)ethan-1-one (8c)}

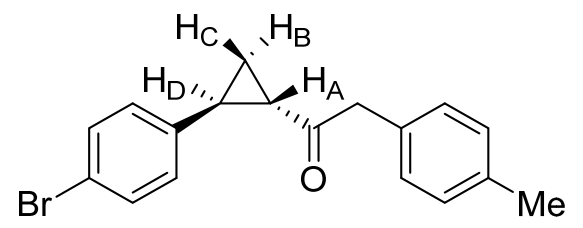

Following the standard procedure B, cyclopropane 8c was isolated as yellow oil, $12.1 \mathrm{mg}, 19 \%$ yield. R: 0.3 in 10\% EtOAc in hexane. GC-MS m/z (\% relative intensity): 105 (31.3), 147 (100), 
160 (29.1), 175 (28.8), 280 (34.5); ${ }^{1} \mathbf{H}$ NMR (500 MHz, $\mathrm{CDCl}_{3}$ ): $\delta 7.35$ (d, $\left.J=8.4 \mathrm{~Hz}, 2 \mathrm{H}\right), 7.14$ (d, $J=8.0 \mathrm{~Hz}, 2 \mathrm{H}), 7.10(\mathrm{~d}, J=8.0 \mathrm{~Hz}, 2 \mathrm{H}), 6.84(\mathrm{~d}, J=8.4 \mathrm{~Hz}, 2 \mathrm{H}), 3.81(\mathrm{~s}, 2 \mathrm{H}), 2.43$ (ddd, $J_{\mathrm{A}-}$ $\left.\mathrm{C}=9.2, J_{\mathrm{A}-\mathrm{B}}=6.6, J_{\mathrm{A}-\mathrm{D}}=4.2 \mathrm{~Hz}, \mathrm{H}_{\mathrm{A}}\right), 2.34(\mathrm{~s}, 3 \mathrm{H}), 2.16\left(\mathrm{ddd}, J_{\mathrm{D}-\mathrm{B}}=8.2, J_{\mathrm{D}-\mathrm{C}}=5.1, J_{\mathrm{D}-\mathrm{A}}=4.2 \mathrm{~Hz}\right.$, $\left.\mathrm{HD}_{\mathrm{D}}\right), 1.66\left(\mathrm{ddd}, J_{\mathrm{C}-\mathrm{A}}=9.2, J_{\mathrm{C}-\mathrm{D}}=5.1, J_{\mathrm{C}-\mathrm{B}}=4.3 \mathrm{~Hz}, \mathrm{HC}_{\mathrm{C}}\right), 1.28\left(\mathrm{ddd}, J_{\mathrm{B}-\mathrm{D}}=8.2, J_{\mathrm{B}-\mathrm{A}}=6.6, J_{\mathrm{B}-\mathrm{C}}=\right.$ $\left.4.3 \mathrm{~Hz}, \mathrm{H}_{\mathrm{B}}\right) ;{ }^{13} \mathbf{C}$ NMR $\left(126 \mathrm{MHz}, \mathrm{CDCl}_{3}\right): \delta 207.0,140.0,137.5,132.2,131.7,130.2,130.1$, $128.8,120.9,51.4,32.2,29.6,21.8,19.6$.

\section{2-Phenyl-1-((1S,2S)-2-phenylcyclopropyl)ethan-1-ol (9)}

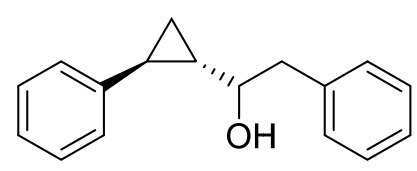

2-Phenyl-1-((IS,2S)-2-phenylcyclopropyl)ethan-1-ol (9) was prepared according to a modified version of a reported procedure. ${ }^{1}$ In a flame-dried $10 \mathrm{~mL}$ RBF, sodium borohydride ( $26.8 \mathrm{mg}, 0.7$ mmol, 1.5 equiv.) was suspended in methanol (1.2 mL). 2-phenyl-1-((1S,2S)-2-phenylcyclopropyl)ethan-1-one (3) (111 mg, $0.472 \mathrm{mmol}, 1$ equiv.) dissolved in methanol (1.2 mL) was added via syringe and stirred for $3 \mathrm{~h}$ at room temperature. After quenching with sat. $\mathrm{NaHCO}_{3}(5$ $\mathrm{mL})$, the aqueous layer was extracted with DCM $(3 \times 3 \mathrm{~mL})$. The combined organic layers were dried over $\mathrm{MgSO}_{4}$ and the crude product was purified by silica-gel column chromatography using $5-20 \%$ EtOAc in hexanes as eluant to afford the product as a colorless oil, $94.9 \mathrm{mg}, 84 \%$ yield (total amount for two separable diastereomers). $\mathbf{R}_{\mathbf{f}}$ (major diastereomer): 0.3 in $20 \%$ EtOAc in hexane; $\mathbf{R}_{\mathbf{f}}$ (minor diastereomer): $\mathbf{R}_{\mathbf{f}}: 0.2$ in $20 \%$ EtOAc in hexane. The two diastereomeric products were determined to be produced in 2.5:1 ratio based on isolated yields. Characterization data for major diastereomer: GC-MS $m / z$ (\% relative intensity): 105 (14.6), 128 (22.3), 129 (14.5), 157 (32.9), 233 (38.8), 234 (100), 235 (18.6); ${ }^{1} \mathbf{H}$ NMR (400 MHz, CDCl ): $\delta 7.37$ - 7.20 (m, 7H), $7.15(\mathrm{dd}, J=7.3,7.3 \mathrm{~Hz}, 1 \mathrm{H}), 7.11-7.03(\mathrm{~m}, 2 \mathrm{H}), 3.45(\mathrm{dd}, J=12.1,7.4 \mathrm{~Hz}, 1 \mathrm{H}), 3.00\left(\mathrm{dd},{ }^{2} J=\right.$ $\left.13.5,{ }^{3} J=4.6 \mathrm{~Hz}, 1 \mathrm{H}\right), 2.88\left(\mathrm{dd},{ }^{2} J=13.6,{ }^{3} J=7.8 \mathrm{~Hz}, 1 \mathrm{H}\right), 2.01-1.90(\mathrm{~m}, 1 \mathrm{H}), 1.35-1.22(\mathrm{~m}$, $1 \mathrm{H}), 0.98-0.83(\mathrm{~m}, 2 \mathrm{H}) ;{ }^{13} \mathbf{C}$ NMR $\left(101 \mathrm{MHz}, \mathrm{CDCl}_{3}\right): \delta 142.6,138.1,129.5,128.5,128.3,126.4$, 125.9, 125.6, 76.0, 43.5, 28.8, 21.0, 13.5.

Rf: 0.2 in $20 \%$ EtOAc in Hexane 


\section{1,2-Diphenyl-1-((1S,2S)-2-phenylcyclopropyl)ethan-1-ol (10)}

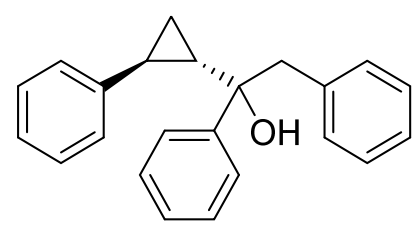

1,2-Diphenyl-1-((1S,2S)-2-phenylcyclopropyl)ethan-1-ol (10) was prepared according to a modified version of a reported procedure. ${ }^{2}$ In a $10 \mathrm{ml}$ flame-dried RBF, 2-phenyl-1-((1S,2S)-2phenylcyclopropyl)ethan-1-one (3) (64.6 mg, $0.273 \mathrm{mmol}, 1$ equiv.) and cerium chloride (202 mg, $0.820 \mathrm{mmol}, 3$ equiv.) were dissolved in dry THF (4 mL). After stirring for $1 \mathrm{~h}$ and cooling to 0 ${ }^{\circ} \mathrm{C}$, a solution of $3 \mathrm{M}$ phenylmagnesium bromide in hexane (273 $\mu 1,0.820 \mathrm{mmol}, 3$ equiv.) was added to the reaction and stirred for $2 \mathrm{~h}$ at $0{ }^{\circ} \mathrm{C}$. The reaction mixture was treated with $10 \%$ aqueous acetic acid $(10 \mathrm{~mL})$, extracted with ether $(3 \times 10 \mathrm{~mL})$, washed with brine $(10 \mathrm{~mL})$ and dried over $\mathrm{MgSO}_{4}$. The combined organic layers were concentrated and the crude residue was purified by silica-gel column chromatography using 10\% EtOAc in hexanes as eluant to afford the product as a colorless oil, $38.6 \mathrm{mg}, 45 \%$ yield. (total amount for two separable diastereomers). $\mathbf{R}_{\mathbf{f}}$ (major diastereomer): 0.3 in 10\% EtOAc in hexane; $\mathbf{R}_{\mathbf{f}}$ (minor diastereomer): 0.2 in 10\% EtOAc in hexane. The two diastereomeric products were determined to be produced in 2.4:1 ratio based on isolated yields. Characterization data for major diastereomer: LC-MS $m / z$ (\% relative intensity): 219 (22), 205 (33), 193 (32), 91 (100) ; ${ }^{1} \mathbf{H}$ NMR (400 MHz, CDCl $)$ ): $\delta 7.45$ (d, J= 7.4 Hz, 1H), $7.34(\mathrm{dd}, J=7.6,7.6 \mathrm{~Hz}, 1 \mathrm{H}), 7.14(\mathrm{dd}, J=9.1,7.2 \mathrm{~Hz}, 1 \mathrm{H}), 7.08(\mathrm{dd}, J=7.3,7.3 \mathrm{~Hz}, 1 \mathrm{H}), 7.00$ $(\mathrm{d}, J=7.1 \mathrm{~Hz}, 1 \mathrm{H}), 6.86(\mathrm{~d}, J=7.2 \mathrm{~Hz}, 1 \mathrm{H}), 3.29(\mathrm{~d}, J=13.3 \mathrm{~Hz}, 1 \mathrm{H}), 3.23(\mathrm{~d}, J=13.3 \mathrm{~Hz}, 1 \mathrm{H})$, $2.03-1.95(\mathrm{~m}, 1 \mathrm{H}), 1.66(\mathrm{ddd}, J=16.5,10.1,5.8 \mathrm{~Hz}, 1 \mathrm{H}), 1.54$ (br, $1 \mathrm{H}) 1.08-0.99$ (m, 1H), 0.86 - $0.78(\mathrm{~m}, 1 \mathrm{H}) ;{ }^{13} \mathrm{C}$ NMR $\left(101 \mathrm{MHz}, \mathrm{CDCl}_{3}\right): \delta 146.3,143.0,136.0,130.7,128.2,128.1,128.0$, $126.9,126.6,126.1,125.8,125.5,125.4,49.3,33.1,19.9,11.6$.

\section{(2-Fluoro-2-((1S,2S)-2-phenylcyclopropyl)ethyl)benzene (11)}

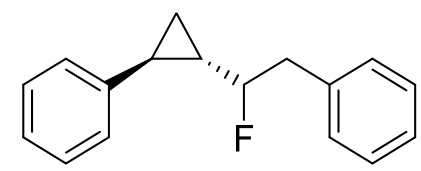

2-phenyl-1-((1S,2S)-2-phenylcyclopropyl)ethan-1-ol (9) (35.3 mg, $0.148 \mathrm{mmol}, 1$ equiv.) and XtalFluor-E (51 mg, $0.222 \mathrm{mmol}, 1.5$ equiv.) were dissolved in dry DCM (1 mL) and DBU (34 $\mu \mathrm{L}, 0.222 \mathrm{mmol}, 1.5$ equiv.) was added at $0{ }^{\circ} \mathrm{C}$. After stirring at room temperature for $12 \mathrm{~h}$, the 
reaction was quenched with $\mathrm{H}_{2} \mathrm{O}(1 \mathrm{~mL})$, extracted with DCM $(3 \times 1 \mathrm{~mL})$, washed with brine (1 $\mathrm{mL}$ ) and dried over $\mathrm{MgSO}_{4}$. The combined organic layers were concentrated and the resulting residue was purified by silica-gel column chromatography using $2 \%$ EtOAc in hexanes as eluant to afford the product as a colorless oil, $17.2 \mathrm{mg}, 79 \%$ yield (total amount for two separable diastereomers). $\mathbf{R}_{\mathbf{f}}$ (major diastereomer): 0.3 in 2\% EtOAc in hexane; $\mathbf{R}_{\mathbf{f}}$ (minor diastereomer): 0.2 in 2\% EtOAc in hexane. The two diastereomeric products were determined to be produced in 2.5:1

ratio based on isolated yields. Characterization data for major diastereomer: GC-MS $\mathrm{m} / \mathrm{z}$ (\% relative intensity): 109 (100), 115 (21), 116 (16.8), 128 (16.5), 129 (39.3), 131 (60.0), 149 (11.3), 240 (14.9); ${ }^{1} \mathbf{H}$ NMR (500 MHz, $\left.\mathrm{CDCl}_{3}\right): \delta 7.40$ - $7.35(\mathrm{~m}, 2 \mathrm{H}), 7.32$ (t, $\left.J=6.2 \mathrm{~Hz}, 3 \mathrm{H}\right), 7.26(\mathrm{dd}$, $J=15.0,7.5 \mathrm{~Hz}, 2 \mathrm{H}), 7.19(\mathrm{dd}, J=7.2,7.2 \mathrm{~Hz}, 1 \mathrm{H}), 7.11(\mathrm{~d}, J=7.5 \mathrm{~Hz}, 2 \mathrm{H}), 5.72-5.64(\mathrm{~m}, 1 \mathrm{H})$, $5.50(\mathrm{ddd}, J=14.8,7.6,7.6 \mathrm{~Hz}, 1 \mathrm{H}), 5.41(\mathrm{dd}, J=7.0,5.8 \mathrm{~Hz}, 1 \mathrm{H}), 3.34(\mathrm{~d}, J=6.7 \mathrm{~Hz}, 2 \mathrm{H}), 2.72$ (dddd, $J=14.6,14.6,7.3,7.3 \mathrm{~Hz}, 1 \mathrm{H}), 2.64-2.53(\mathrm{~m}, 1 \mathrm{H}) ;{ }^{13} \mathbf{C} \mathbf{N M R}\left(126 \mathrm{MHz}, \mathrm{CDCl}_{3}\right): \delta 141.1$, $140.7(\mathrm{~d}, J=19.9 \mathrm{~Hz}), 133.7,129.2,129.2,129.1,129.0,126.8,126.4(\mathrm{~d}, J=6.8 \mathrm{~Hz}), 94.8(\mathrm{~d}, J=$ $172.4 \mathrm{~Hz}), 41.1(\mathrm{~d}, J=24.4 \mathrm{~Hz}), 39.8 ;{ }^{19} \mathbf{F}$ NMR (376 MHz, $\left.\mathrm{CDCl}_{3}\right): \delta-174.31(\mathrm{~m})$.

\section{N-((2-Phenyl-1-((1S,2S)-2-phenylcyclopropyl)ethyl)aniline (12)}

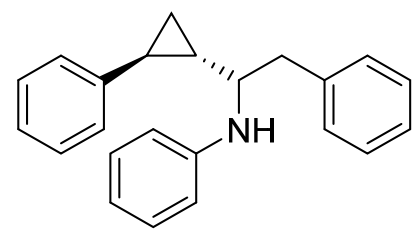

$N$-((2-phenyl-1-((1S,2S)-2-phenylcyclopropyl)ethyl)aniline (12) was prepared according to a modified version of a reported procedure. ${ }^{3}$ 2-Phenyl-1-((1S,2S)-2-phenylcyclopropyl)ethan-1-one (3) (15.7 mg, $0.066 \mathrm{mmol}, 1$ equiv.) and aniline (12.4 mg, $0.133 \mathrm{mmol}, 2$ equiv.) were dissolved in dry THF ( $1 \mathrm{~mL})$. Sodium borohydride $(7.5 \mathrm{mg}, 0.199 \mathrm{mmol}, 3$ equiv.) dissolved in THF ( $1 \mathrm{~mL})$ was added to the reaction. After stirring for $16 \mathrm{~h}$ at room temperature, the reaction mixture was quenched with $1 \mathrm{~N} \mathrm{NaOH}$, extracted with DCM $(3 \times 1 \mathrm{~mL})$ and dried over $\mathrm{MgSO}_{4}$. Combined organic layers were concentrated and the crude residue was purified by silica-gel column chromatography using 10\% EtOAc in hexanes as eluant to afford the product as a colorless oil, 9.4 $\mathrm{mg}, 63 \%$ yield. (total amount for two separable diastereomers). $\mathbf{R}_{\mathbf{f}}$ (major diastereomer): 0.3 in 10\% EtOAc in hexane; $\mathbf{R}_{\mathbf{f}}$ (minor diastereomer): 0.2 in 10\% EtOAc in hexane. The two diastereomeric products were determined to be produced in 2.5:1 ratio based on isolated yields. 
Characterization data for major diastereomer: LC-MS $m / z$ (\% relative intensity): 282 (10), 145 (29), 117 (45), 115 (22), 91 (100); ${ }^{1} \mathbf{H}$ NMR (500 MHz, CDCl $)$ : $\delta 7.35$ - 7.29 (m, 2H), 7.28 $7.22(\mathrm{~m}, 4 \mathrm{H}), 7.20-7.12(\mathrm{~m}, 2 \mathrm{H}), 7.08(\mathrm{~d}, J=7.4,2 \mathrm{H}), 6.77(\mathrm{dd}, J=7.3,7.3 \mathrm{~Hz}, 1 \mathrm{H}), 6.69(\mathrm{~d}, J$ $=8.4 \mathrm{~Hz}, 1 \mathrm{H}), 3.95(\mathrm{ddd}, J=7.7,7.7,4.7 \mathrm{~Hz}, 1 \mathrm{H}), 3.00\left(\mathrm{dd},{ }^{2} J=13.6,{ }^{3} J=4.6 \mathrm{~Hz}, 1 \mathrm{H}\right), 2.88(\mathrm{dd}$, $\left.{ }^{2} J=13.6,{ }^{3} J=7.8 \mathrm{~Hz}, 1 \mathrm{H}\right), 1.99-1.93(\mathrm{~m}, 1 \mathrm{H}), 1.31(\mathrm{ddd}, J=7.8,6.5,4.1 \mathrm{~Hz}, 1 \mathrm{H}), 1.27$ (br, $\left.1 \mathrm{H}\right)$, $0.96-0.85(\mathrm{~m}, 2 \mathrm{H}) ;{ }^{13} \mathbf{C} \mathbf{N M R}\left(126 \mathrm{MHz}, \mathrm{CDCl}_{3}\right): \delta 142.6,138.1,129.5,129.3,128.5,128.3$, $126.4,125.9,125.6,118.6,115.1,76.0,43.5,28.8,21.0,13.5$. One of the quaternary carbon atoms was not detectable.

\section{(S)-2-phenyl-1-((1S,2S)-2-phenylcyclopropyl)propan-1-one (13)}

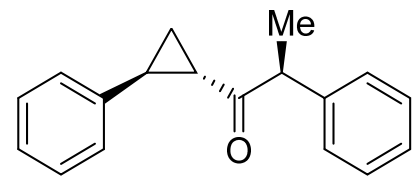

2-phenyl-1-((1S, 2S)-2-phenylcyclopropyl)ethan-1-one (3) (24.4 mg, $0.103 \mathrm{mmol}, 1$ equiv.) was suspended in dry $\mathrm{Et}_{2} \mathrm{O}$ and cooled to $0{ }^{\circ} \mathrm{C}$. A solution of $2 \mathrm{M}$ lithium diisopropylamine in THF (62 $\mu \mathrm{L}, 0.124 \mathrm{mmol}, 1.2$ equiv.) was added dropwise, followed by slow addition of MeI ( $8 \mu \mathrm{L}, 0.134$ mmol, 1.3 equiv.). After stirring at $0{ }^{\circ} \mathrm{C}$ for $12 \mathrm{~h}$, the reaction was quenched with $\mathrm{H}_{2} \mathrm{O}(1 \mathrm{~mL})$, extracted with DCM (3 x $1 \mathrm{~mL})$, washed with brine $(1 \mathrm{~mL})$ and dried over $\mathrm{MgSO}_{4}$. Combined organic layers were concentrated and the resulting residue was purified by silica-gel column chromatography using 2\% EtOAc in hexane as eluant to afford the product as a colorless oil, 20 $\mathrm{mg}, 78 \%$ yield (total amount for two separable diastereomers). $\mathbf{R}_{\mathbf{f}}$ (major diastereomer): 0.2 in $2 \%$ EtOAc in hexane; $\mathbf{R}_{\mathbf{f}}$ (minor diastereomer): 0.1 in 2\% EtOAc in hexane. The two diastereomeric products were determined to be produced in 2.5:1 ratio based on isolated yields. Characterization data for major diastereomer: GC-MS $m / z$ (\% relative intensity): 105 (67.9), 115 (32.6), 117 (54.9), 127 (58.1), 145 (100), 146 (17.6), 250 (18.9); ${ }^{1} \mathbf{H}$ NMR (500 MHz, $\left.\mathrm{CDCl}_{3}\right): \delta 7.33$ (dd, $J=7.4$, $7.4 \mathrm{~Hz}, 2 \mathrm{H}), 7.30-7.16(\mathrm{~m}, 6 \mathrm{H}), 6.99$ (d, $J=7.4 \mathrm{~Hz}, 2 \mathrm{H}), 3.91$ (q, $J=6.9 \mathrm{~Hz}, 1 \mathrm{H}), 2.50$ (ddd, $J$ $=9.1,6.6,4.1 \mathrm{~Hz}, 1 \mathrm{H}), 2.13-2.08(\mathrm{~m}, 1 \mathrm{H}), 1.63-1.58(\mathrm{~m}, 1 \mathrm{H}), 1.42(\mathrm{~d}, J=6.9 \mathrm{~Hz}, 3 \mathrm{H}), 1.22$ (ddd, $J=8.0,6.6,4.2 \mathrm{~Hz}, 1 \mathrm{H}) ;{ }^{13} \mathbf{C}$ NMR (126 MHz, $\left.\mathrm{CDCl}_{3}\right): \delta 209.5,141.4,129.7,129.2,128.9$, $127.8,127.2,126.8,106.2,54.7,32.5,30.4,20.1,18.4$. The major diastereomer was determined to correspond to $(S)$-2-phenyl-1-((1S,2S)-2-phenylcyclopropyl)propan-1-one upon comparison with $7 \mathbf{i}$. 


\section{2-Fluoro-2-phenyl-1-((1S,2S)-2-phenylcyclopropyl)ethan-1-one (14)}<smiles>O=C([C@H]1C[C@H]1c1ccccc1)[C@H](F)c1ccccc1</smiles>

2-fluoro-2-phenyl-1-((1S,2S)-2-phenylcyclopropyl)ethan-1-one (14) was prepared according to a modified version of a reported procedure. ${ }^{4}$ 2-phenyl-1-((1S, 2S)-2-phenylcyclopropyl)ethan-1-one (3) $(19.3 \mathrm{mg}, 0.082 \mathrm{mmol}, 1$ equiv.) and sodium dodecyl benzenesulfonate (23.6 mg, $0.082 \mathrm{mmol}$, 1 equiv.) were suspended in $\mathrm{H}_{2} \mathrm{O}(1 \mathrm{~mL})$. SelectF (31.8 mg, $0.090 \mathrm{mmol}, 1.1$ equiv.) was added at $80{ }^{\circ} \mathrm{C}$ and stirred for $16 \mathrm{~h}$ at $80^{\circ} \mathrm{C}$. After extraction with DCM $(3 \times 1 \mathrm{~mL})$, washing with brine (1 $\mathrm{mL}$ ) and drying over $\mathrm{MgSO}_{4}$, combined organic layers were concentrated and the resulting residue was purified by silica-gel column chromatography using $0.5 \%$ methanol in DCM as eluant to afford the product as a colorless oil, $20.2 \mathrm{mg}, 78 \%$ (total amount for two separable diastereomers). $\mathbf{R}_{\mathbf{f}}$ (major diastereomer): 0.3 in $0.5 \%$ methanol in DCM; $\mathbf{R}_{\mathbf{f}}$ (minor diastereomer): 0.2 in $0.5 \%$ methanol in DCM. The two diastereomeric products were determined to be produced in 1.8:1 ratio based on isolated yields. Characterization data for major diastereomer: The major isomer is labeled A and the minor B. GC-MS $m / z$ (\% relative intensity): 109 (26.3), 115 (19.0), 117 (100), 121 (68), 134 (22.2), 135 (15.8), 145 (17.2), 177 (62.3), 266 (3.9); ${ }^{1} \mathbf{H}$ NMR (500 MHz, CDCl ): $\delta 7.41$ (s, 5H), $7.30-7.24(\mathrm{~m}, 2 \mathrm{H}), 7.24-7.19(\mathrm{~m}, 1 \mathrm{H}), 7.02(\mathrm{~d}, J=7.4 \mathrm{~Hz}, 2 \mathrm{H}), 5.82(\mathrm{~d}, J=48.7 \mathrm{~Hz}, 1 \mathrm{H})$, $2.57-2.46(\mathrm{~m}, 2 \mathrm{H}), 1.80-1.75(\mathrm{~m}, 1 \mathrm{H}), 1.52-1.46(\mathrm{~m}, 1 \mathrm{H}) ;{ }^{13} \mathbf{C} \mathbf{N M R}\left(126 \mathrm{MHz}, \mathrm{CDCl}_{3}\right): \delta$ 205.0, 140.2, 130.3, 129.7, 129.3, 127.6, 127.5, 127.0, 96.7 (d, J=188.0 HZ), 31.7, 28.9, 19.9. Carbonyl carbon atom and one of the quaternary carbon atoms were not detectable; ${ }^{19} \mathbf{F}$ NMR (376 $\left.\mathrm{MHz}, \mathrm{CDCl}_{3}\right) \delta-180.30(\mathrm{~d}, J=48.6 \mathrm{~Hz})$. The major isomer is tentatively assigned to $(S)$-2-fluoro2-phenyl-1-((1S,2S)-2-phenylcyclopropyl)ethan-1-one based on diastereoselectivity of the $\alpha$ functionalization reaction to obtain $\mathbf{1 3}$.

\section{1-Phenyl-2-((1S,2S)-2-phenylcyclopropyl)ethane-1,2-dione (15)}<smiles>O=C(C(=O)[C@@H]1C[C@H]1c1ccccc1)c1ccccc1</smiles>

2-Phenyl-1-((1S, 2S)-2-phenylcyclopropyl)ethan-1-one (3) (20 mg, $0.085 \mathrm{mmol}, 1 \mathrm{equiv}$.$) and$ copper (II) bromide (2.4 mg, $0.017 \mathrm{mmol}, 0.2$ equiv.) were dissolved in DMSO (1 mL). After stirring for 10 minutes and addition of thiomorpholine (26 mg, $0.25 \mathrm{mmol}, 3$ equiv.), the reaction 
mixture was stirred for $12 \mathrm{~h}$ open to the air at room temperature. The reaction mixture was purified by silica-gel column chromatography using $10 \%$ EtOAc in hexanes as eluant to afford the product as a colorless oil, $20.0 \mathrm{mg}$, $94 \%$ yield. $\mathbf{R}_{\mathbf{f}}$ : 0.3 in $10 \%$ EtOAc in hexanes. GC-MS $\mathrm{m} / z$ (\% relative intensity): 105 (100), 115 (19.6), 117 (20.6), 127 (27.7), 145 (51.6), 250 (3.4); ${ }^{1}$ H NMR (400 $\left.\mathrm{MHz}, \mathrm{CDCl}_{3}\right): \delta 8.03(\mathrm{dd}, J=8.3,1.2 \mathrm{~Hz}, 2 \mathrm{H}), 7.69-7.61(\mathrm{~m}, 1 \mathrm{H}), 7.51(\mathrm{dd}, J=10.8,4.6 \mathrm{~Hz}$, $2 \mathrm{H}), 7.35-7.27(\mathrm{~m}, 2 \mathrm{H}), 7.27-7.20(\mathrm{~m}, 1 \mathrm{H}), 7.17(\mathrm{dd}, J=5.2,3.2 \mathrm{~Hz}, 2 \mathrm{H}), 2.90-2.77(\mathrm{~m}, 2 \mathrm{H})$, 1.95 (ddd, $J=9.3,5.3,4.3 \mathrm{~Hz}, 1 \mathrm{H}), 1.68$ (ddd, $J=8.1,6.9,4.2 \mathrm{~Hz}, 1 \mathrm{H}) ;{ }^{13} \mathbf{C}$ NMR $(101 \mathrm{MHz}$, $\left.\mathrm{CDCl}_{3}\right): \delta 200.3,191.8,139.3,134.6,132.1,130.3,128.8,128.6,126.9,126.3,31.6,29.8,20.8$.

\section{3-(Dimethylamino)-2-phenyl-1-((1S,2S)-2-phenylcyclopropyl)prop-2-en-1-one (16)}

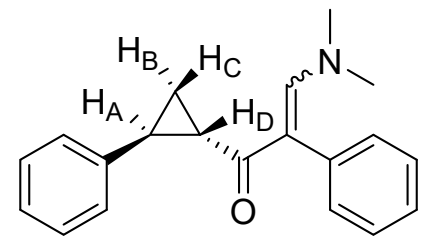

3-(Dimethylamino)-2-phenyl-1-((1S,2S)-2-phenylcyclopropyl)prop-2-en-1-one (16) was prepared according to a modified version of a reported procedure. ${ }^{5}$ 2-Phenyl-1-((1S,2S)-2phenylcyclopropyl)ethan-1-one (3) (16.9 mg, $0.072 \mathrm{mmol}, 1$ equiv.) was dissolved in dimethylformamide dimethyl acetal $(0.4 \mathrm{~mL}, 0.143 \mathrm{mmol}, 2$ equiv. $)$ and stirred at $90{ }^{\circ} \mathrm{C}$ for $16 \mathrm{~h}$. The reaction was purified by silica-gel column chromatography using $2 \%$ methanol in DCM as eluant to afford the product as a colorless oil, $18.2 \mathrm{mg}, 87 \%$ (total amount for two separable $E / Z$ diastereomers). $\mathbf{R}_{\mathbf{f}}$ (major diastereomer): 0.3 in $2 \%$ methanol in DCM; $\mathbf{R}_{\mathbf{f}}$ (minor diastereomer): 0.2 in $2 \%$ methanol in DCM. The two diastereomeric products were determined to be produced in 15:1 ratio based on isolated yields. Characterization data for major diastereomer: GC-MS $m / z$ ( $\%$ relative intensity): 102 (16.6), 103 (16.6), 105 (15.9), 115 (42.1) 116 (64.6) 117 (23.9), 130 (19.0) 131 (54.0), 144 (42.7), 146 (23.1), 147 (23.4), 157 (22.9), 158 (27.9), 170 (22), 172 (20.8), 173 (28.3), 174 (48.3), 186 (100), 187 (70.2), 247 (21.3), 248 (39.2), 291 (84.3), 292 (20.4); ${ }^{1} \mathbf{H}$ NMR $\left(500 \mathrm{MHz}, \mathrm{CDCl}_{3}\right): \delta 7.65(\mathrm{~s}, 1 \mathrm{H}), 7.26-7.12(\mathrm{~m}, 7 \mathrm{H}), 7.11-7.05(\mathrm{~m}, 1 \mathrm{H}), 6.91(\mathrm{~d}, J=7.1,2 \mathrm{H})$, $2.69(\mathrm{~s}, 6 \mathrm{H}), 2.46\left(\mathrm{ddd}, J_{\mathrm{D}-\mathrm{B}}=9.1, J_{\mathrm{D}-\mathrm{C}}=6.2, J_{\mathrm{D}-\mathrm{B}}=4.3 \mathrm{~Hz}, \mathrm{H}_{\mathrm{D}}\right), 1.93\left(\mathrm{ddd}, J_{\mathrm{A}-\mathrm{C}}=8.2, J_{\mathrm{A}-\mathrm{B}}=5.2\right.$, $\left.J_{\mathrm{A}-\mathrm{C}}=4.4 \mathrm{~Hz}, \mathrm{HA}_{\mathrm{A}}\right), 1.64\left(\mathrm{ddd},, J_{\mathrm{B}-\mathrm{D}}=9.0, J_{\mathrm{B}-\mathrm{A}}=5.3, J_{\mathrm{B}-\mathrm{C}}=3.7 \mathrm{~Hz}, \mathrm{HB}\right), 1.01\left(\mathrm{ddd}, J_{\mathrm{C}-\mathrm{A}}=8.2, J_{\mathrm{C}-}\right.$ $\left.\mathrm{D}=6.3, J_{\mathrm{C}-\mathrm{B}}=3.7 \mathrm{~Hz}, \mathrm{HC}\right) ;{ }^{13} \mathbf{C} \mathbf{~ N M R}\left(126 \mathrm{MHz}, \mathrm{CDCl}_{3}\right): \delta 196.9,149.6,142.5,138.5,133.2$, 
$128.9,128.6,127.3,127.0,126.5,111.8,29.9,29.0,19.4$. Carbonyl carbon atom was not detectable.

\section{Trimethyl((2-phenyl-1-((1S,2S)-2-phenylcyclopropyl)vinyl)oxy)silane (17)}

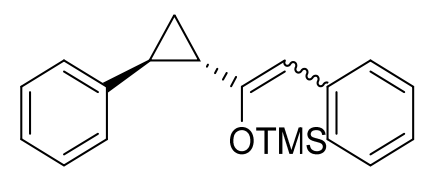

2-phenyl-1-((1S, 2S)-2-phenylcyclopropyl)ethan-1-one (3) (22.8 mg, $0.097 \mathrm{mmol}, 1$ equiv.) was suspended in dry $\mathrm{Et}_{2} \mathrm{O}$ and cooled to $0^{\circ} \mathrm{C}$. A solution of $2 \mathrm{M}$ Lithium diisopropylamine in THF (58 $\mu \mathrm{L}, 0.116 \mathrm{mmol}, 1.2$ equiv.) was added dropwise, followed by slow addition of TMSCl (16 $\mu \mathrm{L}$, $0.125 \mathrm{mmol}, 1.3$ equiv.). After stirring at $0{ }^{\circ} \mathrm{C}$ for $12 \mathrm{~h}$, the reaction was concentrated in vacuo and was purified by silica-gel column chromatography using 10\% EtOAc in hexane as eluant to afford the product as a colorless oil, $25.6 \mathrm{mg}, 86 \%$ (total amount for two inseparable $E / Z$ diastereomers). $\mathbf{R}_{\mathbf{f}}: 0.2$ in $10 \%$ EtOAc in hexane. The two $E / Z$ diastereomeric products were determined to be $4: 1$ ratio based on NMR. The major isomer is labeled A and the minor B. GC-MS $m / z$ (\% relative intensity): 115 (25.1), 201 (44.3), 202 (24.9), 203 (25.8), 204 (13.7), 216 (47.7), 217 (100), 218 (35.4), 308 (62.3), 309 (17.4); ${ }^{1} \mathbf{H}$ NMR (500 MHz, $\left.\mathrm{CDCl}_{3}\right): \delta 7.48$ (d, J= $\left.7.4 \mathrm{~Hz}, 2 \mathrm{H}\right), 7.30$ $7.22(\mathrm{~m}, 5 \mathrm{H}), 7.20(\mathrm{~d}, J=4.4 \mathrm{~Hz}, 1 \mathrm{H}), 7.16(\mathrm{dd}, J=13.3,5.9 \mathrm{~Hz}, 1 \mathrm{H}), 7.13-7.04(\mathrm{~m}, 4 \mathrm{H}), 5.86$ $(\mathrm{s}, 0.2 \mathrm{H}, \mathbf{B}), 5.48(\mathrm{~s}, 1 \mathrm{H}), 2.29-2.24(\mathrm{~m}, 0.3 \mathrm{H}, \mathbf{B}), 2.20(\mathrm{dd}, J=8.6,5.7 \mathrm{~Hz}, 0.3 \mathrm{H}, \mathbf{B}), 2.18-2.13$ (m, 1H, A), 1.77 (ddd, $J=8.8,5.5,5.5 \mathrm{~Hz}, 1 \mathrm{H}, \mathbf{A}), 1.43(\mathrm{dd}, J=10.0,4.5 \mathrm{~Hz}, 0.3 \mathrm{H}, \mathbf{B}), 1.41-$ $1.36(\mathrm{~m}, 1 \mathrm{H}, \mathbf{A}), 1.18$ (ddd, $J=8.7,5.4,5.4 \mathrm{~Hz}, 1 \mathrm{H}, \mathbf{A}), 1.07-1.03$ (m, 0.3H, B), 0.29 (s, 2.2H, B), 0.12 (s, 9H, A); ${ }^{13} \mathbf{C}$ NMR (126 MHz, CDCl 3 ): $\delta 153.4$ (B), 153.4 (A), 142.8 (A), 142.7 (B), 137.8 (B), 137.4 (A), 130.3 (B), 129.5 (A), 129.2 (A), 129.1 (B), 128.9 (A), 128.8 (B), 127.0 (B), 126.7 (A), 126.6 (B), 126.4 (A), 126.3 (A), 126.1 (B), 109.2 (B), 106.8 (A), 29.8 (A), 25.8 (A), 25.4 (B), 23.7 (B), 16.6 (A), 15.3 (B), 1.6 (A), 1.2 (B). 


\section{2-Phenyl-3-((1S,2S)-2-phenylcyclopropyl)quinoxaline (18)}<smiles>c1ccc(-c2nc3ccccc3nc2[C@H]2C[C@H]2c2ccccc2)cc1</smiles>

3-(Dimethylamino)-2-phenyl-1-((1S,2S)-2-phenylcyclopropyl)prop-2-en-1-one (18) was prepared according to a modified version of a reported procedure. ${ }^{6}$ 1-Phenyl-2-((1S,2S)-2phenylcyclopropyl)ethane-1,2-dione (15) $(34.0 \mathrm{mg}, 0.136 \mathrm{mmol}, 1$ equiv.) and potassium tertbutoxide ( $0.6 \mathrm{mg}, 0.4$ equiv.) were suspended in methanol $(1 \mathrm{~mL})$. After addition of benzne-1,2diamine (14.7 $\mathrm{mg}, 0.136 \mathrm{mmol}, 1$ equiv.), the reaction solution was stirred for $12 \mathrm{~h}$ at room temperature. The resulting mixture was concentrated and purified by silica-gel column chromatography using 20\% EtOAc in hexanes as eluant to afford the product as a colorless oil, 39.9 mg, 91\% yield, 0\% ee. $\mathbf{R}_{\mathbf{f}}: 0.3$ in 20\% EtOAc in hexanes. LC-MS $m / z$ (\% relative intensity): 323 (100), 324 (28); ${ }^{1} \mathbf{H}$ NMR (500 MHz, $\left.\mathrm{CDCl}_{3}\right): \delta 8.11(\mathrm{~d}, J=8.0 \mathrm{~Hz}, 1 \mathrm{H}), 8.03$ (d, $J=8.0 \mathrm{~Hz}$, 1H), $7.76-7.63(\mathrm{~m}, 4 \mathrm{H}), 7.41(\mathrm{~d}, J=6.8 \mathrm{~Hz}, 3 \mathrm{H}), 7.28(\mathrm{t}, J=7.3 \mathrm{~Hz}, 2 \mathrm{H}), 7.19$ (dd, $J=7.1,7.1$ $\mathrm{Hz}, 1 \mathrm{H}), 7.11(\mathrm{~d}, J=7.4 \mathrm{~Hz}, 2 \mathrm{H}), 2.84-2.76(\mathrm{~m}, 1 \mathrm{H}), 2.67-2.59(\mathrm{~m}, 1 \mathrm{H}), 2.23-2.15(\mathrm{~m}, 1 \mathrm{H})$, $1.56-1.50(\mathrm{~m}, 1 \mathrm{H}) ;{ }^{13} \mathbf{C} \mathbf{N M R}\left(126 \mathrm{MHz}, \mathrm{CDCl}_{3}\right): \delta 155.6,155.0,142.2,142.2,141.2,139.2$ $130.3,130.3,130.0,129.7,129.5,129.2,129.2,126.8,126.7,31.4,28.2,20.6$. 


\section{X-ray Crystallographic Analysis}

X-ray crystal diffraction data was collected using a XtaLab Synergy-S Dualflex diffractometer equipped with a HyPix-6000HE HPC area detector for data collection at 100.00(10) K. A preliminary set of cell constants and an orientation matrix was calculated from a small sampling of reflections. A short pre-experiment was run, from which an optimal data collection strategy was determined. The full data collection was carried out using a PhotonJet $(\mathrm{Cu}) \mathrm{X}$-ray Source with a frame time of 0.05 seconds and a detector distance of $31.2 \mathrm{~mm}$. Series of frames were collected in $0.50^{\circ}$ steps in $\omega$ at different $2 \theta, \kappa$, and $\phi$ settings. After the intensity data were corrected for absorption, the final cell constants were calculated from the xyz centroids of 14400 strong reflections from the actual data collection after integration. See Table S3-4 for additional crystal and refinement information. The structure was solved using $\mathrm{ShelXT}^{7}$ and refined using ShelXL. ${ }^{8}$ The space group $P 212121$ was determined based on systematic absences. Most or all non-hydrogen atoms were assigned from the solution. Full-matrix least squares / difference Fourier cycles were performed which located any remaining non-hydrogen atoms. All non-hydrogen atoms were refined with anisotropic displacement parameters. All hydrogen atoms were placed in ideal positions and refined as riding atoms with relative isotropic displacement parameters. The final full matrix least squares refinement converged to $R 1=0.0307\left(F^{2}, I>2 \sigma(I)\right)$ and $w R 2=0.0836$ $\left(F^{2}\right.$, all data). 
Table S4. Crystal data and structure refinement for 1-((1S, 2S)-2-(4-bromophenyl)cyclopropyl)2-phenylethan-1-one (5d). Cambridge Crystallographic Data Centre (CCDC) entry: 050794.

\begin{tabular}{|c|c|c|}
\hline Empirical formula & \multicolumn{2}{|l|}{$\mathrm{C}_{17} \mathrm{H}_{15} \mathrm{BrO}$} \\
\hline Formula weight & \multicolumn{2}{|l|}{315.20} \\
\hline Temperature & \multicolumn{2}{|l|}{$100.00(10) \mathrm{K}$} \\
\hline Wavelength & \multicolumn{2}{|l|}{$1.54184 \AA$} \\
\hline Crystal system & \multicolumn{2}{|l|}{ orthorhombic } \\
\hline Space group & \multicolumn{2}{|l|}{$P 212121$} \\
\hline \multirow[t]{3}{*}{ Unit cell dimensions } & $a=5.8732(2) \AA$ & $\alpha=90^{\circ}$ \\
\hline & $b=7.3387(3) \AA$ & $\beta=90^{\circ}$ \\
\hline & $c=32.1290(14) \AA$ & $\gamma=90^{\circ}$ \\
\hline Volume & \multicolumn{2}{|l|}{$1384.81(10) \AA^{3}$} \\
\hline$Z$ & \multicolumn{2}{|l|}{4} \\
\hline Density (calculated) & \multicolumn{2}{|l|}{$1.512 \mathrm{Mg} / \mathrm{m}^{3}$} \\
\hline Absorption coefficient & \multicolumn{2}{|l|}{$3.940 \mathrm{~mm}^{-1}$} \\
\hline$F(000)$ & \multicolumn{2}{|l|}{640} \\
\hline Crystal color, morphology & \multicolumn{2}{|l|}{ colourless, needle } \\
\hline Crystal size & \multicolumn{2}{|c|}{$0.256 \times 0.149 \times 0.067 \mathrm{~mm}^{3}$} \\
\hline Theta range for data collection & \multicolumn{2}{|l|}{2.751 to $82.168^{\circ}$} \\
\hline Index ranges & \multicolumn{2}{|c|}{$-7 \leq h \leq 7,-7 \leq k \leq 9,-40 \leq l \leq 38$} \\
\hline Reflections collected & \multicolumn{2}{|l|}{16745} \\
\hline Independent reflections & \multicolumn{2}{|c|}{$2907[R($ int $)=0.0568]$} \\
\hline Observed reflections & \multicolumn{2}{|l|}{2830} \\
\hline Completeness to theta $=74.504^{\circ}$ & \multicolumn{2}{|l|}{$99.5 \%$} \\
\hline Absorption correction & \multicolumn{2}{|l|}{ Multi-scan } \\
\hline Max. and min. transmission & \multicolumn{2}{|c|}{1.00000 and 0.70207} \\
\hline Refinement method & \multicolumn{2}{|c|}{ Full-matrix least-squares on $F^{2}$} \\
\hline Data / restraints / parameters & \multicolumn{2}{|l|}{$2907 / 0$ / 172} \\
\hline Goodness-of-fit on $F^{2}$ & \multicolumn{2}{|l|}{1.101} \\
\hline Final $R$ indices $[I>2 \operatorname{sigma}(I)]$ & \multicolumn{2}{|c|}{$R 1=0.0307, w R 2=0.0830$} \\
\hline$R$ indices (all data) & \multicolumn{2}{|c|}{$R 1=0.0364, w R 2=0.0836$} \\
\hline Absolute structure parameter & \multicolumn{2}{|l|}{$-0.026(12)$} \\
\hline Largest diff. peak and hole & \multicolumn{2}{|c|}{0.483 and -0.734 e. $\AA^{-3}$} \\
\hline
\end{tabular}


Table S5. Crystal data and structure refinement for 1-((1S,2S)-2-(4-bromophenyl)cyclopropyl)-2(p-tolyl)ethan-1-one (8c). Cambridge Crystallographic Data Centre (CCDC) entry: 2050795.

\begin{tabular}{|c|c|c|}
\hline Empirical formula & \multicolumn{2}{|l|}{$\mathrm{C} 18 \mathrm{H} 17 \mathrm{Br} \mathrm{O}$} \\
\hline Formula weight & \multicolumn{2}{|l|}{329.22} \\
\hline Temperature & \multicolumn{2}{|l|}{$100.00(10) \mathrm{K}$} \\
\hline Wavelength & \multicolumn{2}{|l|}{$1.54184 \AA$} \\
\hline Crystal system & \multicolumn{2}{|l|}{ monoclinic } \\
\hline Space group & \multicolumn{2}{|l|}{$P 2{ }_{1}$} \\
\hline \multirow[t]{3}{*}{ Unit cell dimensions } & $a=7.38540(10) \AA$ & $\square=90^{\circ}$ \\
\hline & $b=5.85390(10) \AA$ & $\square=93.6740(10)^{\circ}$ \\
\hline & $c=17.0708(2) \AA$ & $\square=90^{\circ}$ \\
\hline Volume & \multicolumn{2}{|l|}{$736.512(18) \AA^{3}$} \\
\hline$Z$ & \multicolumn{2}{|l|}{2} \\
\hline Density (calculated) & \multicolumn{2}{|l|}{$1.485 \mathrm{Mg} / \mathrm{m}^{3}$} \\
\hline Absorption coefficient & \multicolumn{2}{|l|}{$3.729 \mathrm{~mm}^{-1}$} \\
\hline$F(000)$ & \multicolumn{2}{|l|}{336} \\
\hline Crystal color, morphology & \multicolumn{2}{|l|}{ colourless, plate } \\
\hline Crystal size & \multicolumn{2}{|c|}{$0.257 \times 0.184 \times 0.049 \mathrm{~mm}^{3}$} \\
\hline Theta range for data collection & \multicolumn{2}{|l|}{2.594 to $77.776^{\circ}$} \\
\hline Index ranges & \multicolumn{2}{|c|}{$-8 \square h \square 9,-7 \square k \square 7,-21 \square l \square 21$} \\
\hline Reflections collected & \multicolumn{2}{|l|}{24493} \\
\hline Independent reflections & \multicolumn{2}{|c|}{$3103[R(\mathrm{int})=0.0918]$} \\
\hline Observed reflections & \multicolumn{2}{|l|}{3069} \\
\hline Completeness to theta $=74.504^{\circ}$ & \multicolumn{2}{|l|}{$100.0 \%$} \\
\hline Absorption correction & \multicolumn{2}{|l|}{ Multi-scan } \\
\hline Max. and min. transmission & \multicolumn{2}{|c|}{1.00000 and 0.81146} \\
\hline Refinement method & \multicolumn{2}{|c|}{ Full-matrix least-squares on $F^{2}$} \\
\hline Data / restraints / parameters & \multicolumn{2}{|l|}{$3103 / 1 / 182$} \\
\hline Goodness-of-fit on $F^{2}$ & \multicolumn{2}{|l|}{1.130} \\
\hline Final $R$ indices $[I>2 \operatorname{sigma}(I)]$ & \multicolumn{2}{|c|}{$R 1=0.0338, w R 2=0.0890$} \\
\hline$R$ indices (all data) & \multicolumn{2}{|c|}{$R 1=0.0342, w R 2=0.0892$} \\
\hline Absolute structure parameter & \multicolumn{2}{|l|}{$-0.020(19)$} \\
\hline Largest diff. peak and hole & \multicolumn{2}{|c|}{0.594 and $-0.637 \mathrm{e} . \AA^{-3}$} \\
\hline
\end{tabular}




\section{NMR Spectra}

1-diazo-3-phenyl-2-propanone (2), ${ }^{1} \mathrm{H} \mathrm{NMR}\left(500 \mathrm{MHz}, \mathrm{CDCl}_{3}\right)$ :

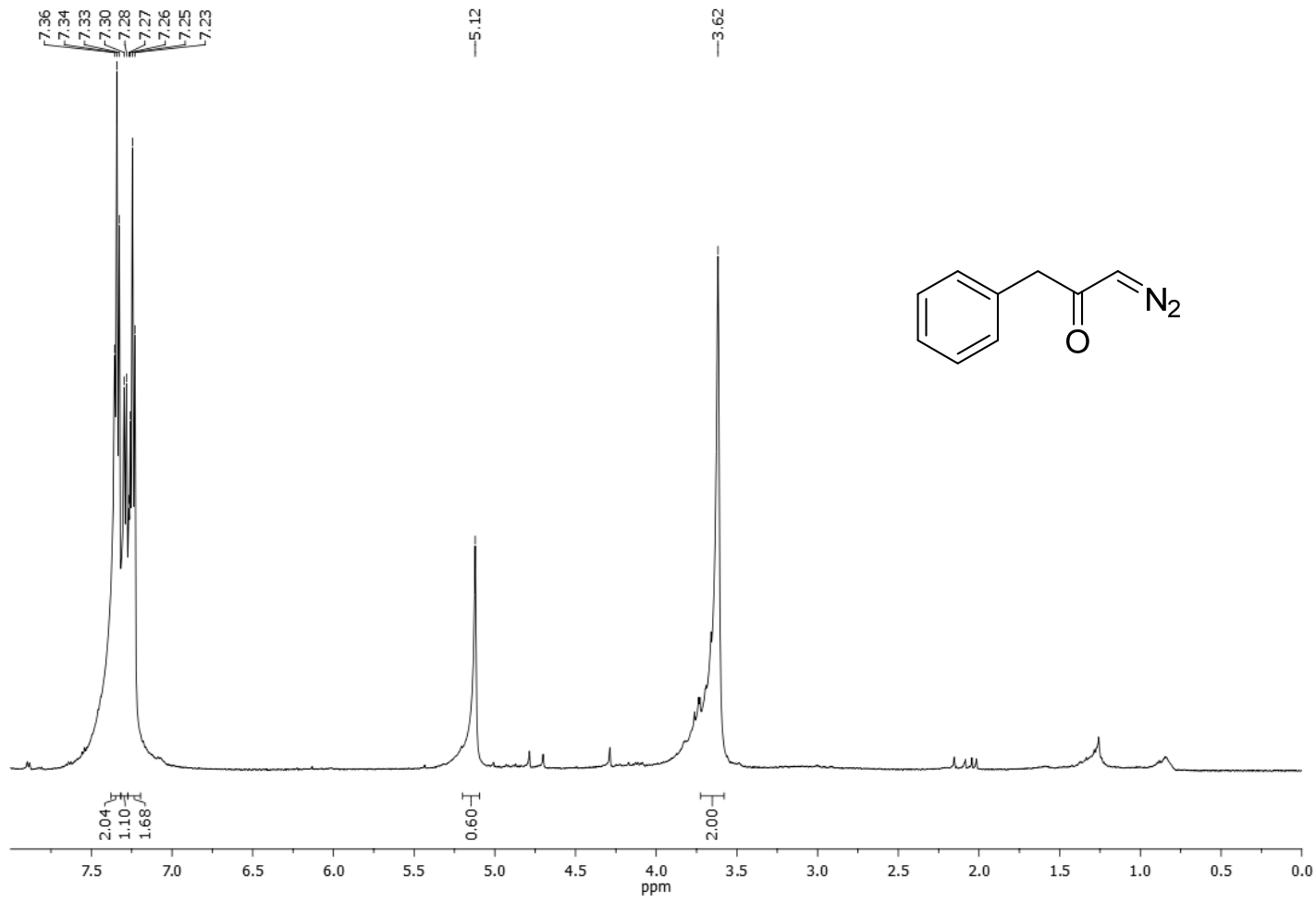

1-diazo-3-phenyl-2-propanone (2), $\left.{ }^{13} \mathrm{C} \mathrm{NMR} \mathrm{(126} \mathrm{MHz,} \mathrm{CDCl}_{3}\right)$ :
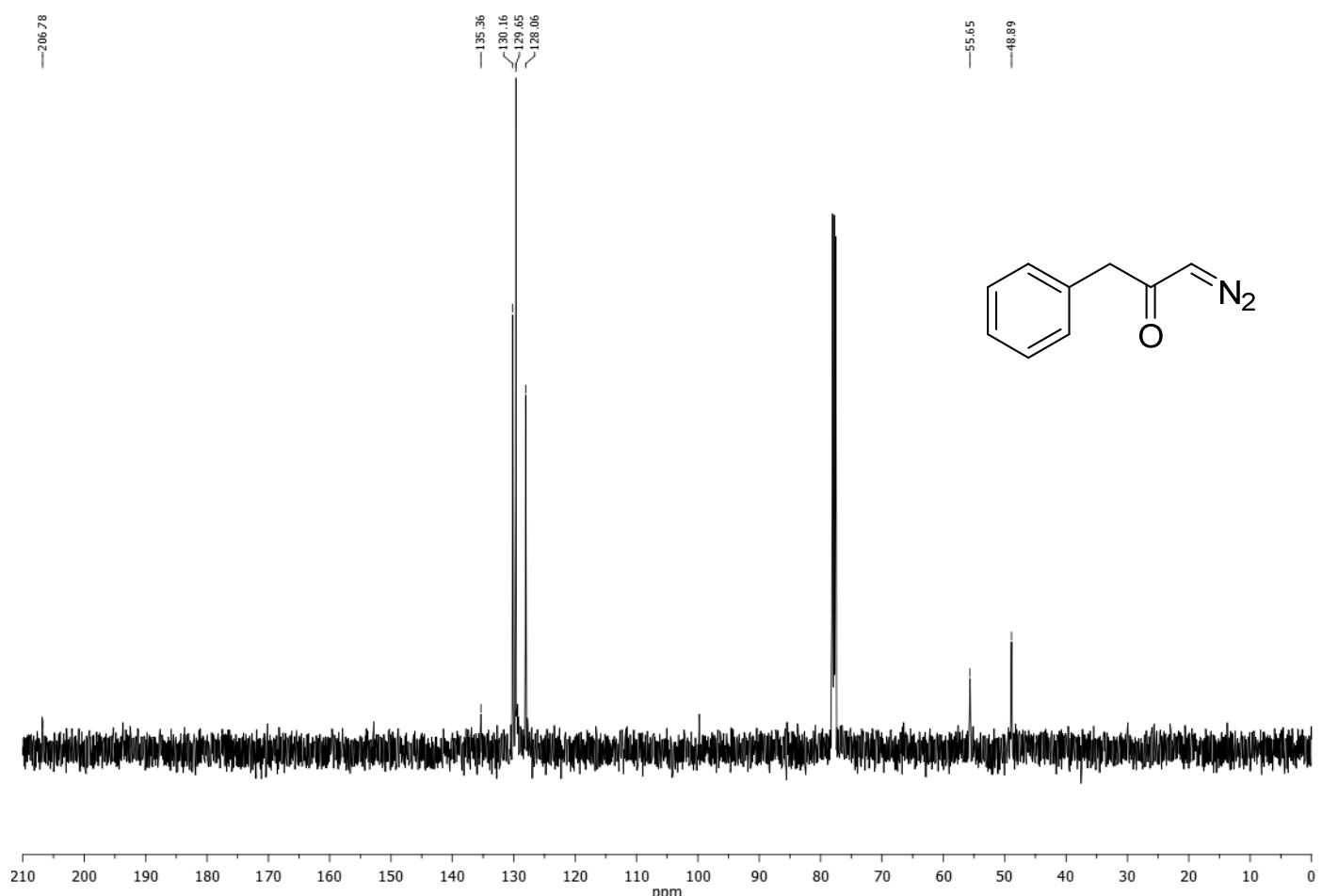
1-(4-chloro-phenyl)-3-diazo-propan-2-one (6a), ${ }^{1} \mathrm{H} \mathrm{NMR}\left(500 \mathrm{MHz}, \mathrm{CDCl}_{3}\right)$ :

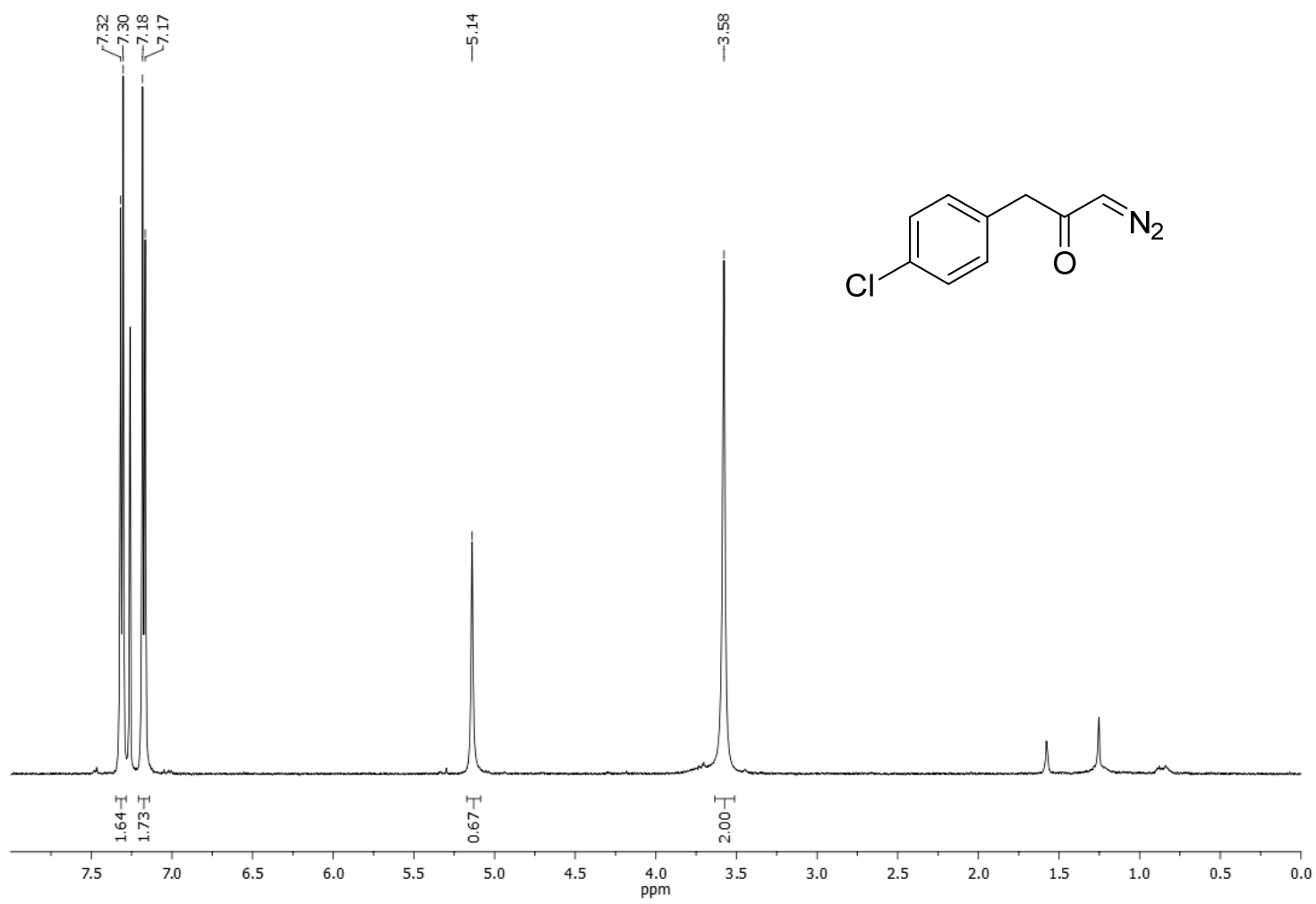

1-(4-chloro-phenyl)-3-diazo-propan-2-one (6a), ${ }^{13} \mathrm{C} \mathrm{NMR}\left(126 \mathrm{MHz}, \mathrm{CDCl}_{3}\right)$ :

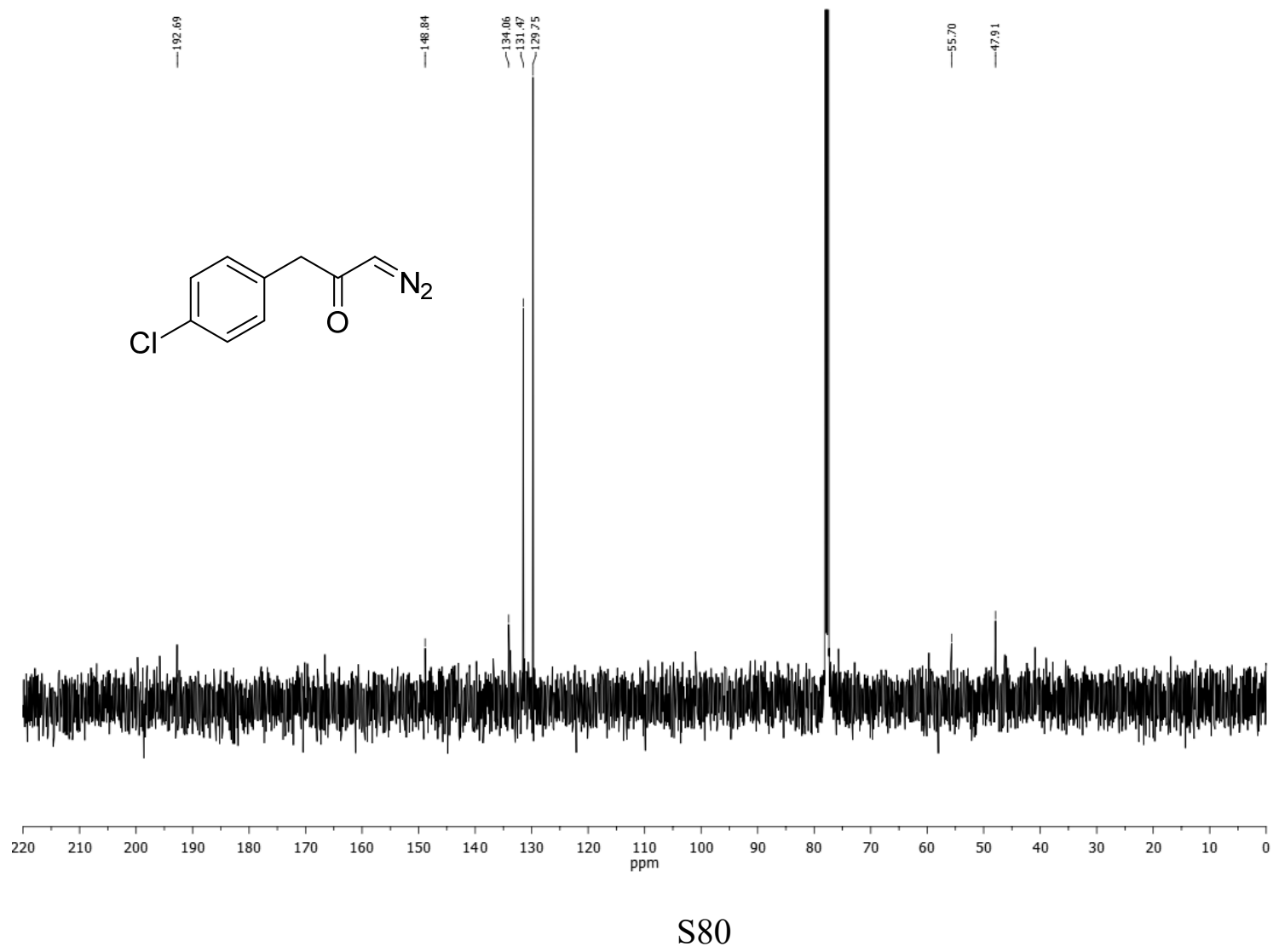


1-(4-bromo-phenyl)-3-diazo-propan-2-one (6b), ${ }^{1} \mathrm{H}$ NMR (500 MHz, $\left.\mathrm{CDCl}_{3}\right)$ :

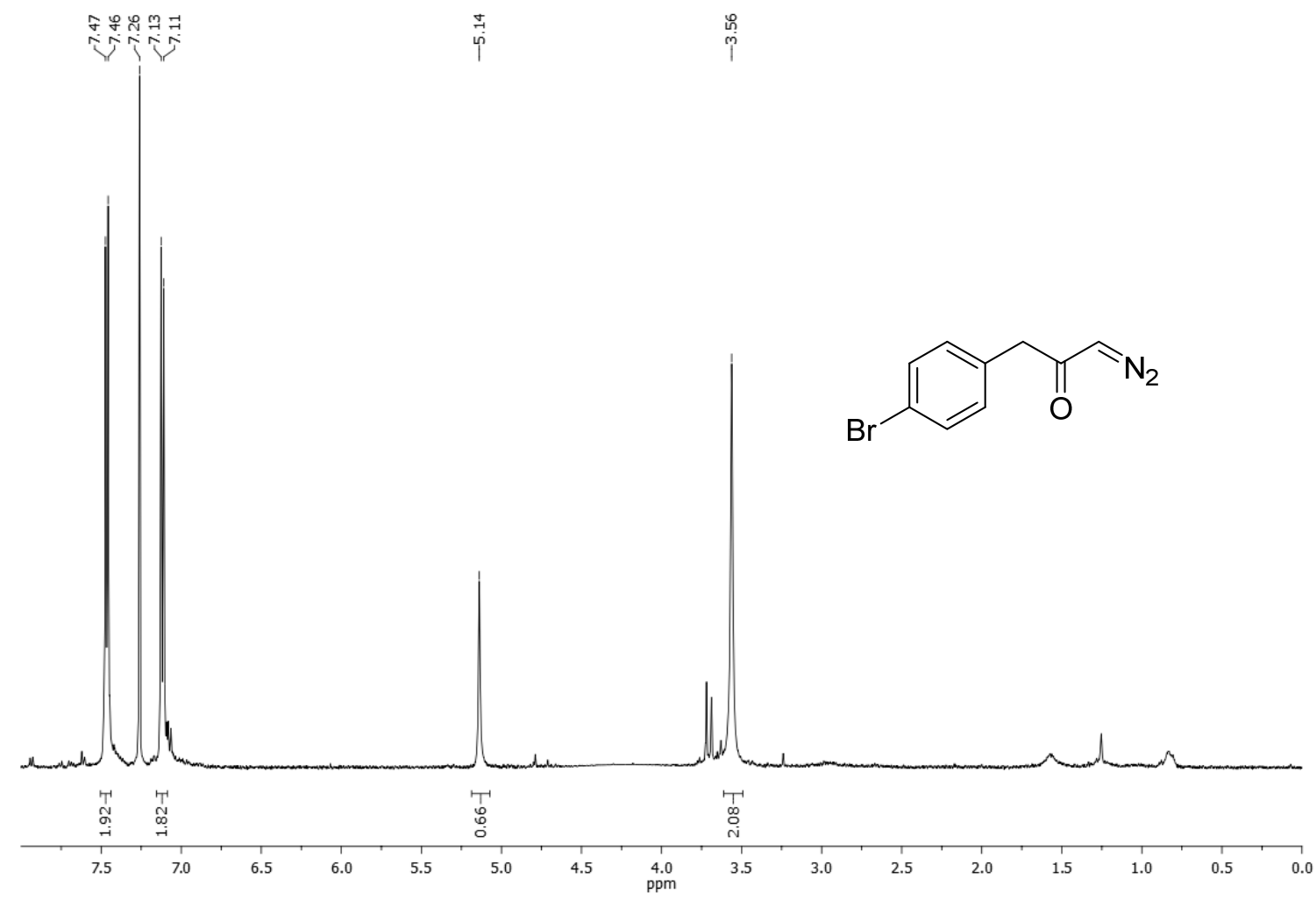

1-(4-bromo-phenyl)-3-diazo-propan-2-one (6b), ${ }^{13} \mathrm{C} \mathrm{NMR}\left(126 \mathrm{MHz}, \mathrm{CDCl}_{3}\right)$ :

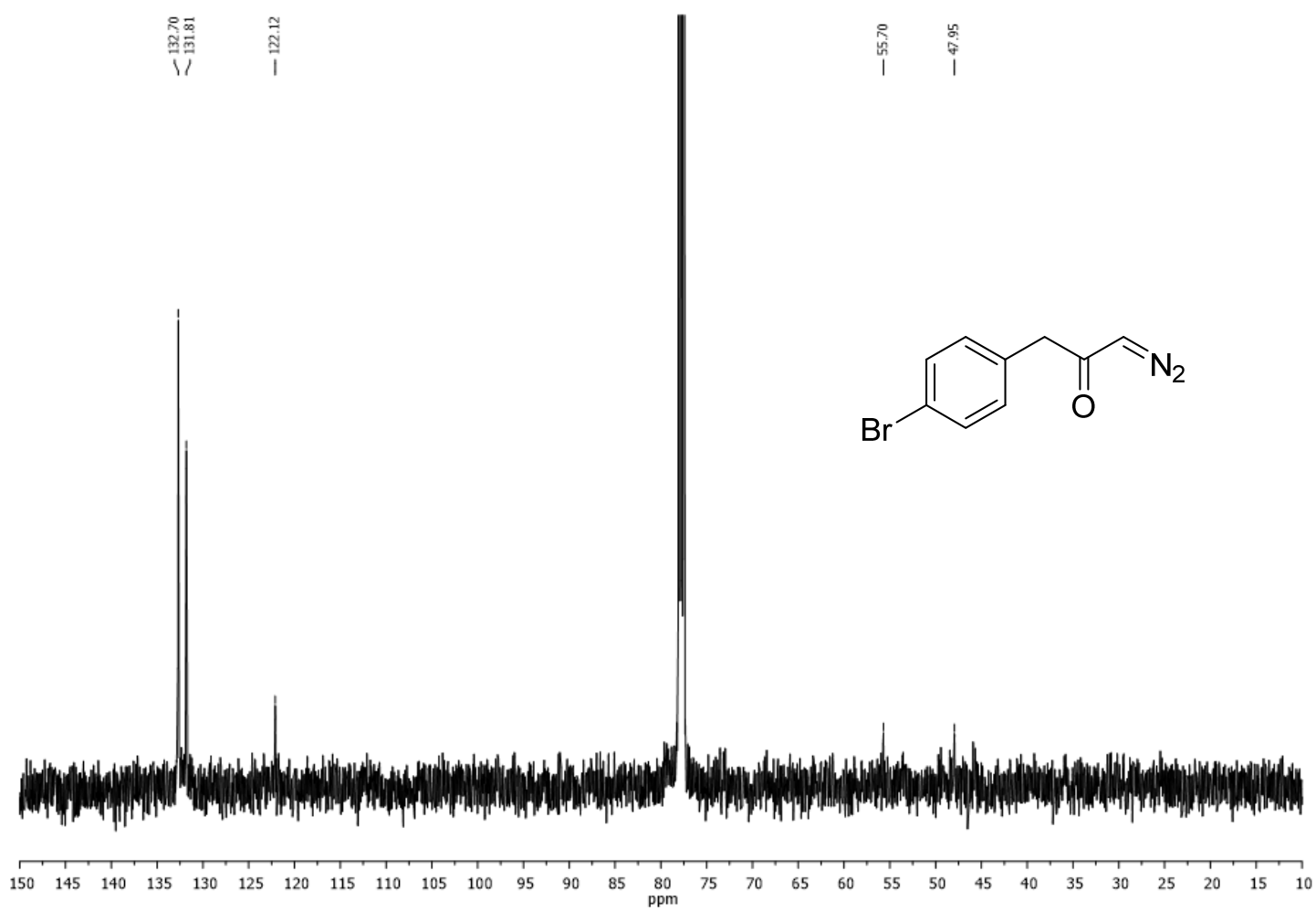


1-(3-bromo-phenyl)-3-diazo-propan-2-one (6c), ${ }^{1} \mathrm{H}$ NMR (500 MHz, $\left.\mathrm{CDCl}_{3}\right)$ :

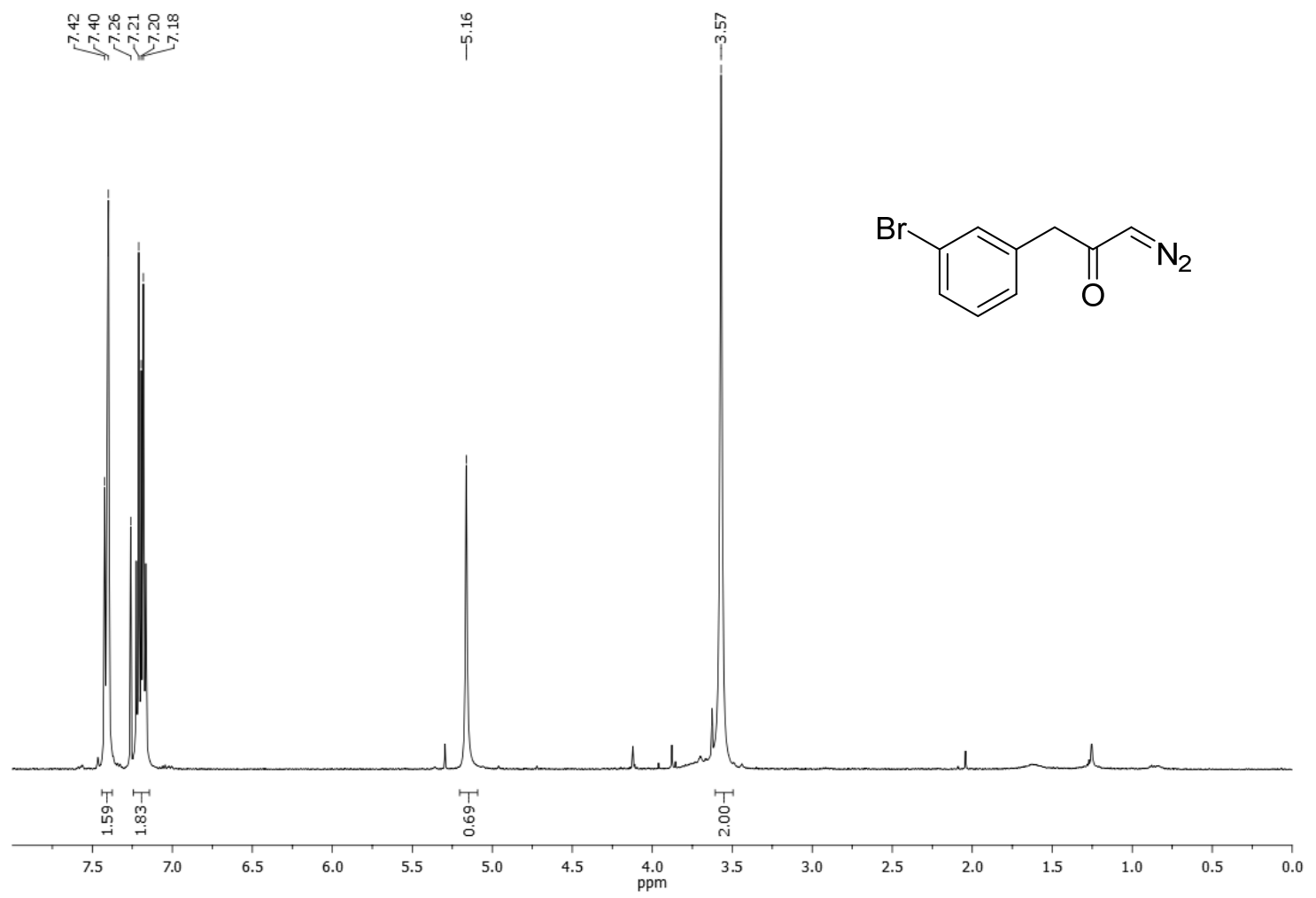

1-(3-bromo-phenyl)-3-diazo-propan-2-one (6c), $\left.{ }^{13} \mathrm{C} \mathrm{NMR} \mathrm{(126} \mathrm{MHz,} \mathrm{CDCl}_{3}\right)$ :
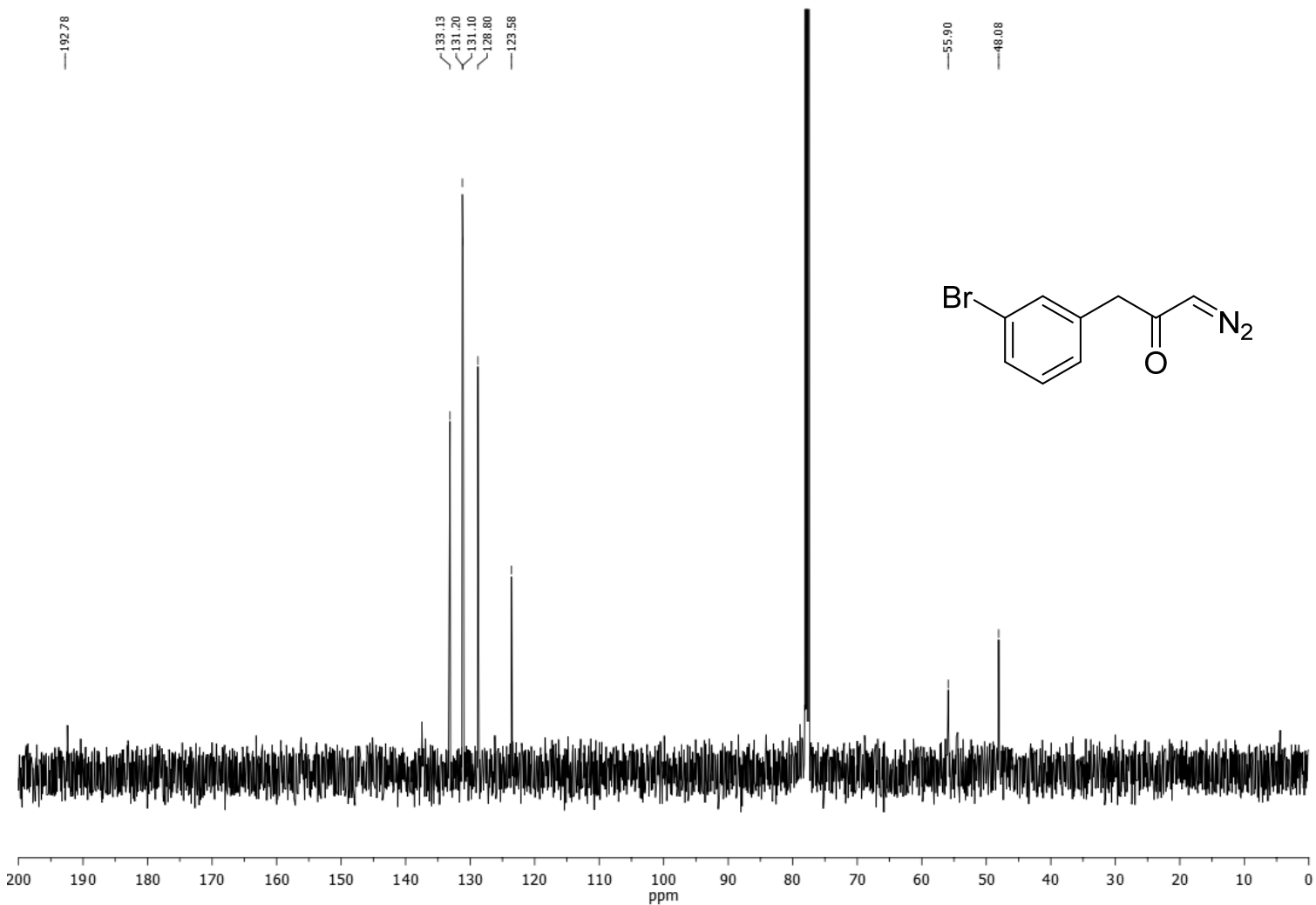
1-(2-bromo-phenyl)-3-diazo-propan-2-one (6d), ${ }^{1} \mathrm{H} \mathrm{NMR}\left(500 \mathrm{MHz}, \mathrm{CDCl}_{3}\right)$ :

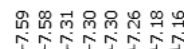
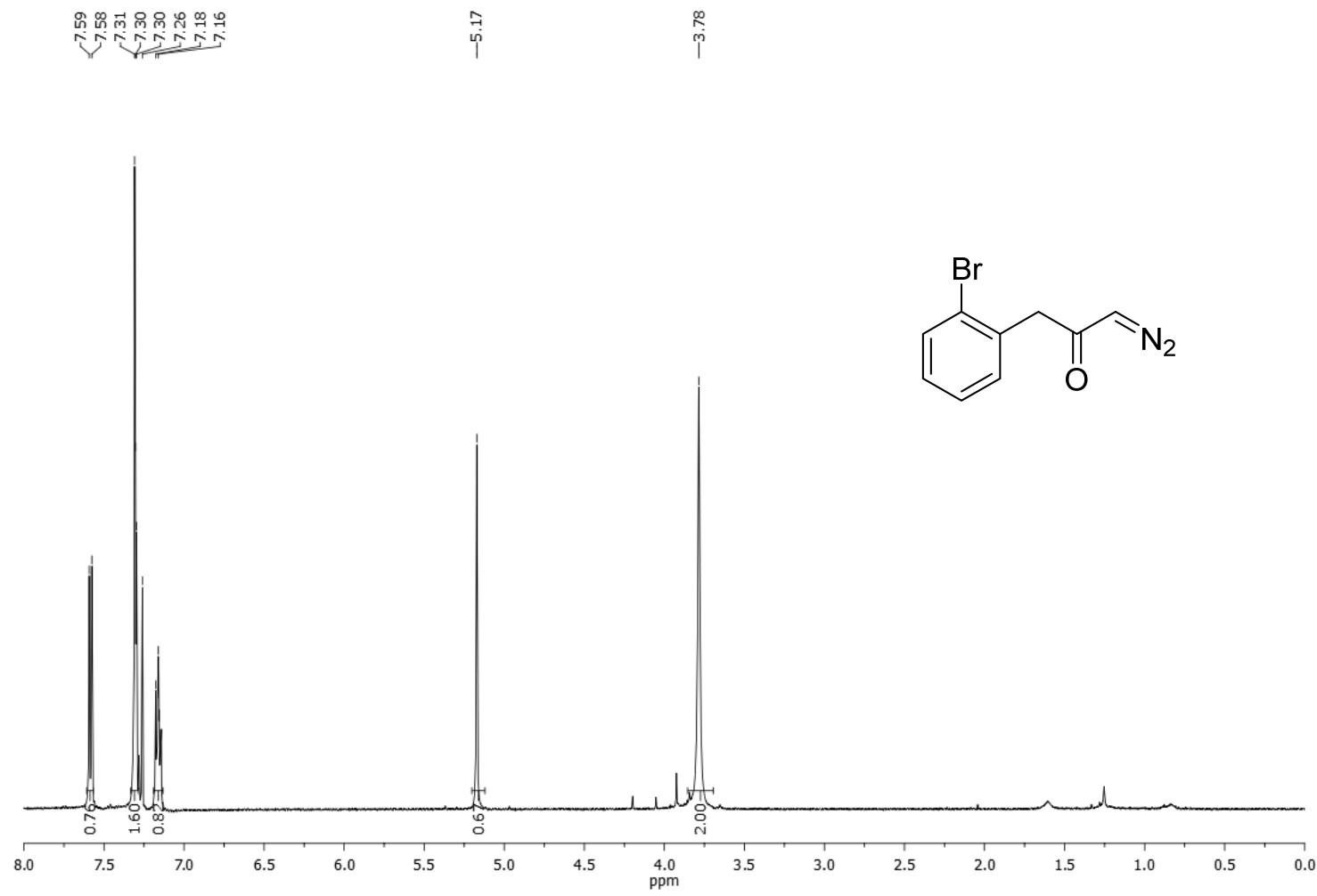

1-(2-bromo-phenyl)-3-diazo-propan-2-one (6d), ${ }^{13} \mathrm{C} \mathrm{NMR}\left(126 \mathrm{MHz}, \mathrm{CDCl}_{3}\right)$ :

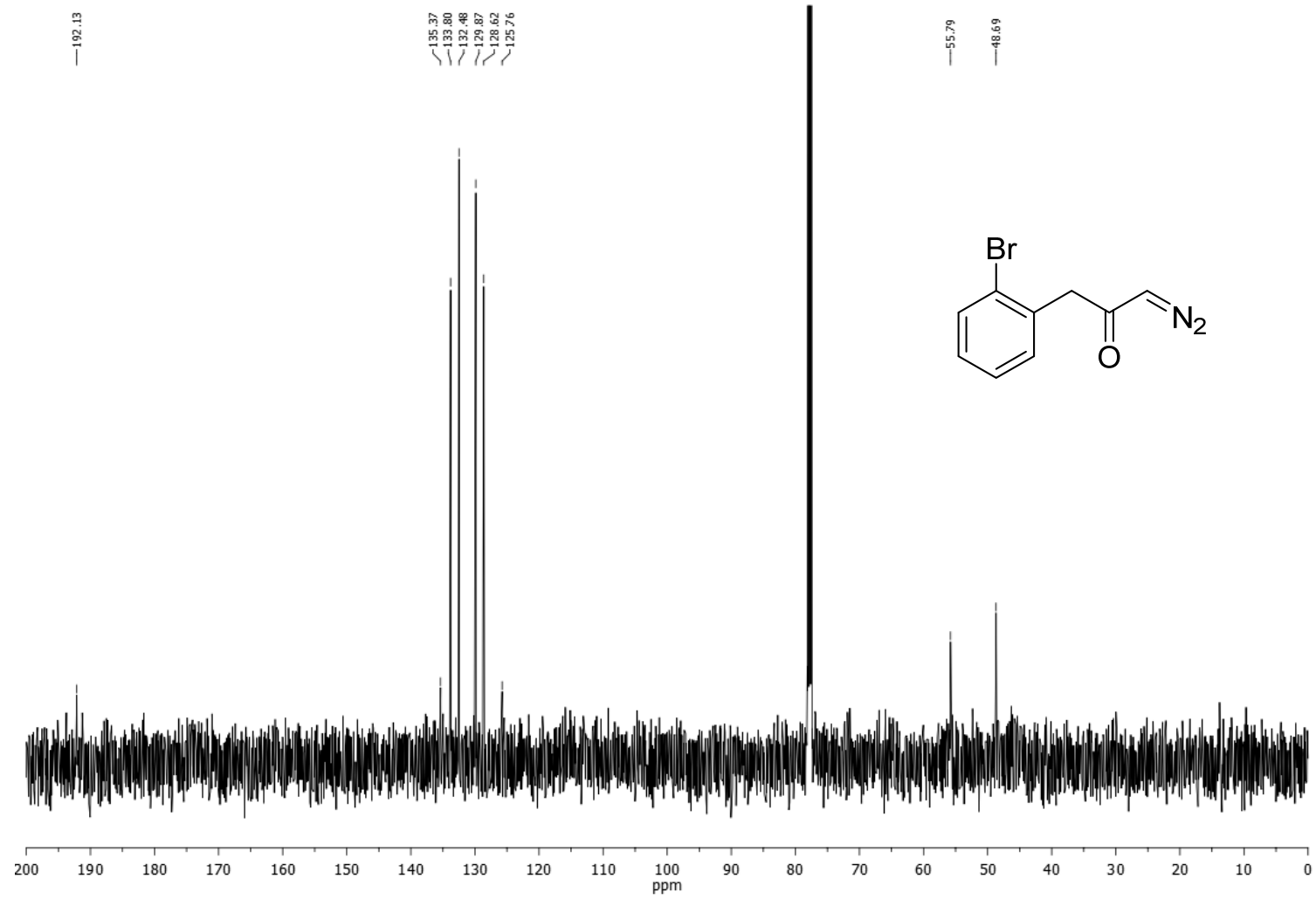


1-diazo-3-(4-methylphenyl)-2-propanone (6e), ${ }^{1} \mathrm{H} \mathrm{NMR}\left(500 \mathrm{MHz}, \mathrm{CDCl}_{3}\right)$ :

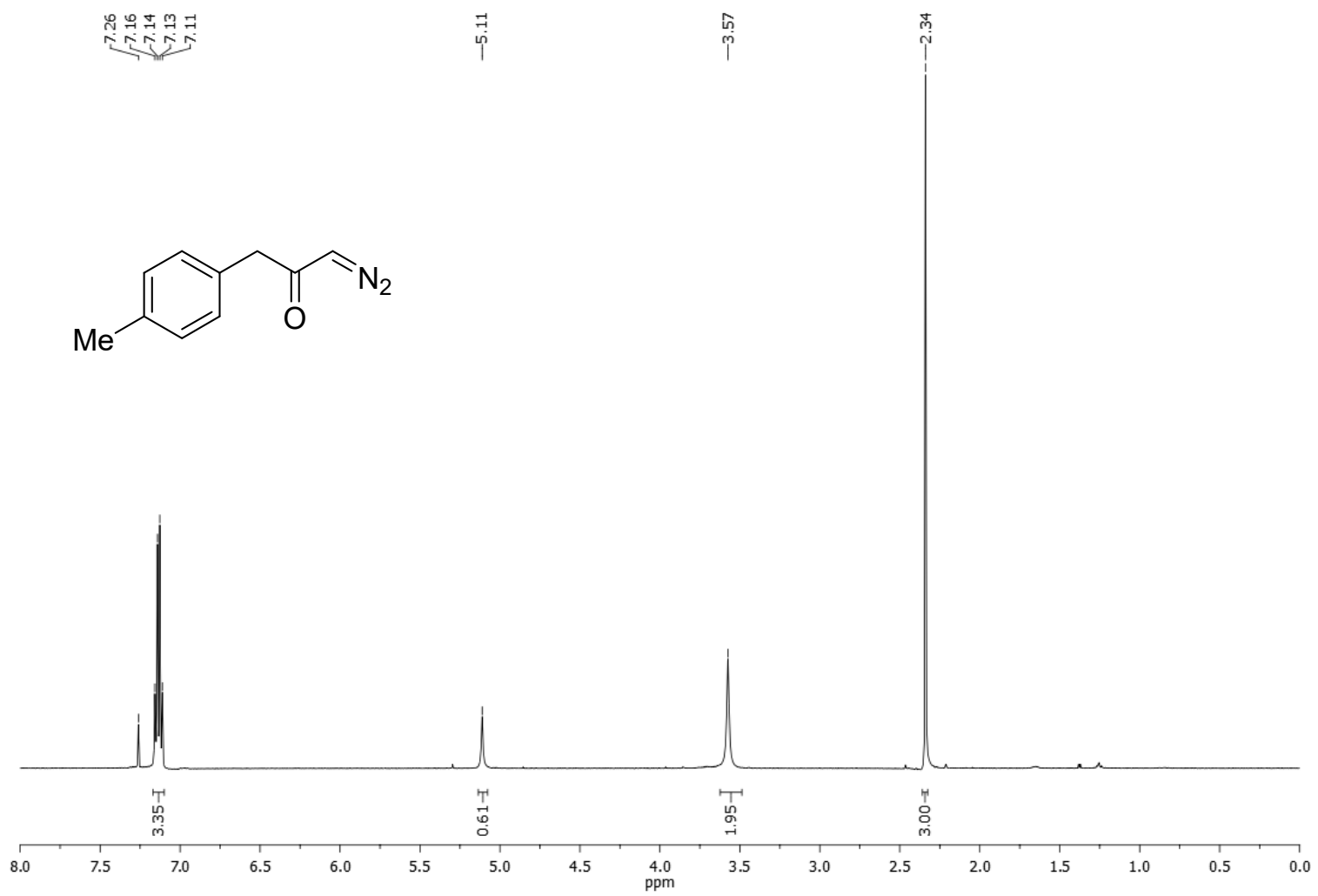

1-diazo-3-(4-methylphenyl)-2-propanone (6e), ${ }^{13} \mathrm{C} \mathrm{NMR}\left(126 \mathrm{MHz}, \mathrm{CDCl}_{3}\right)$ :

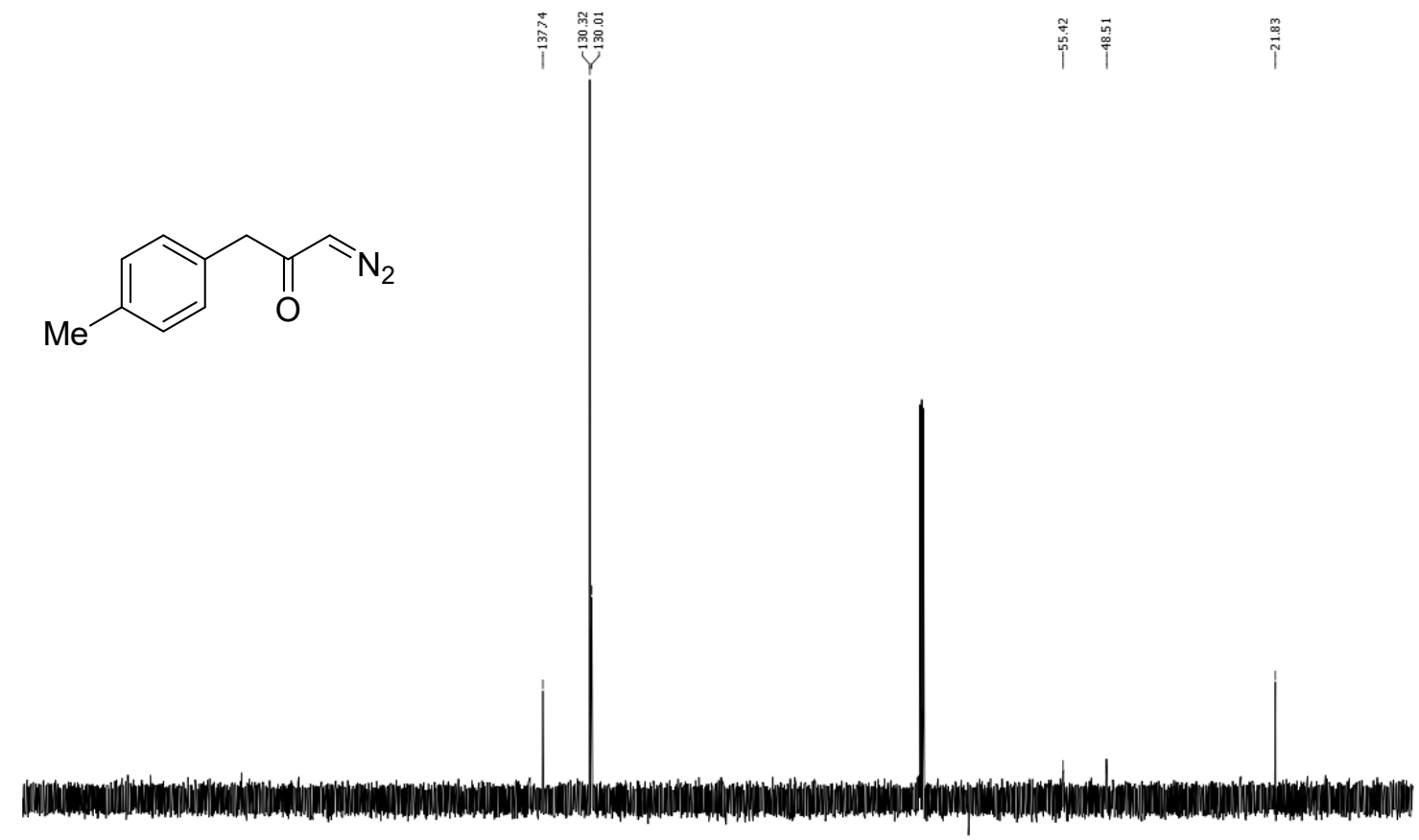

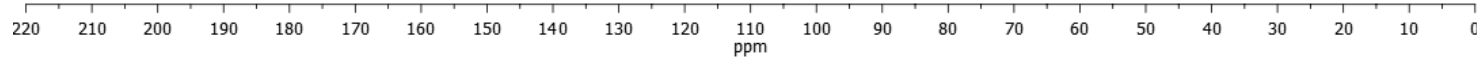


1-diazo-3-(4-methoxyphenyl)-2-propanone (6f), ${ }^{1} \mathrm{H}$ NMR (500 MHz, $\mathrm{CDCl}_{3}$ ):

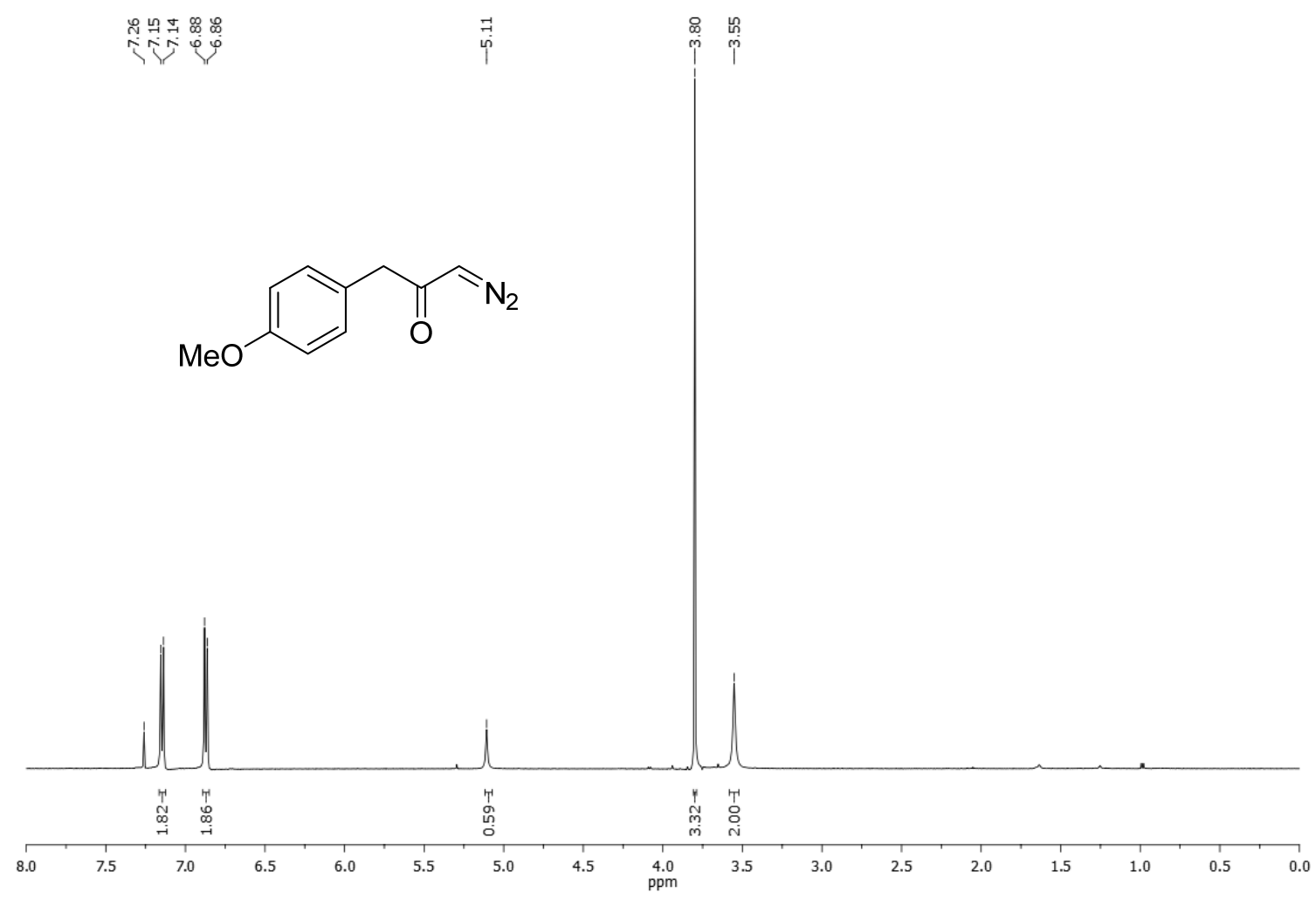

1-diazo-3-(4-methoxyphenyl)-2-propanone (6f), ${ }^{13} \mathrm{C} \mathrm{NMR}\left(126 \mathrm{MHz}, \mathrm{CDCl}_{3}\right)$ :

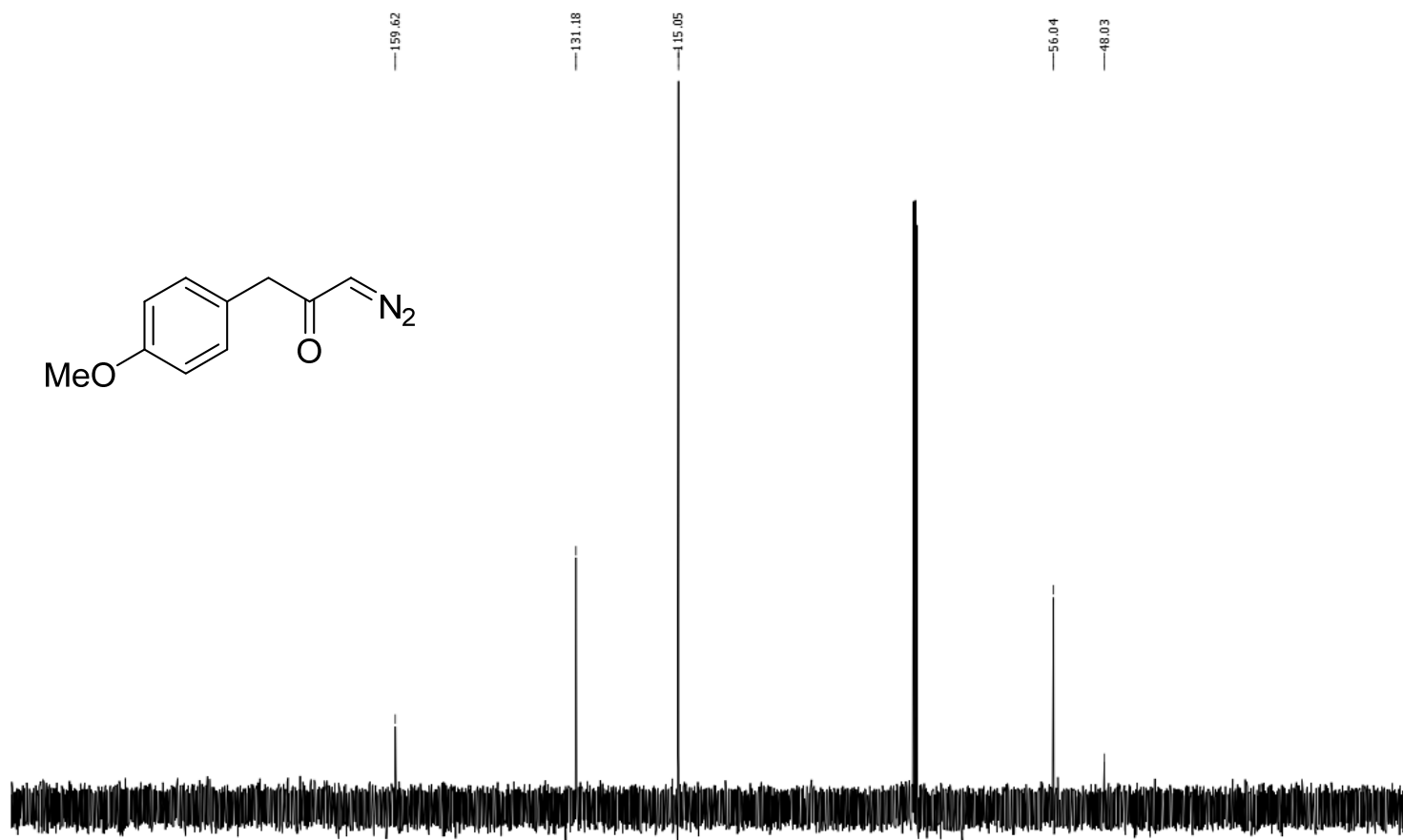

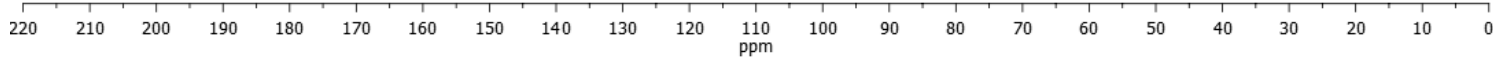


1-diazo-3-(3-methoxyphenyl)-2-propanone (6g), ${ }^{1} \mathrm{H}$ NMR (500 MHz, $\left.\mathrm{CDCl}_{3}\right)$ :

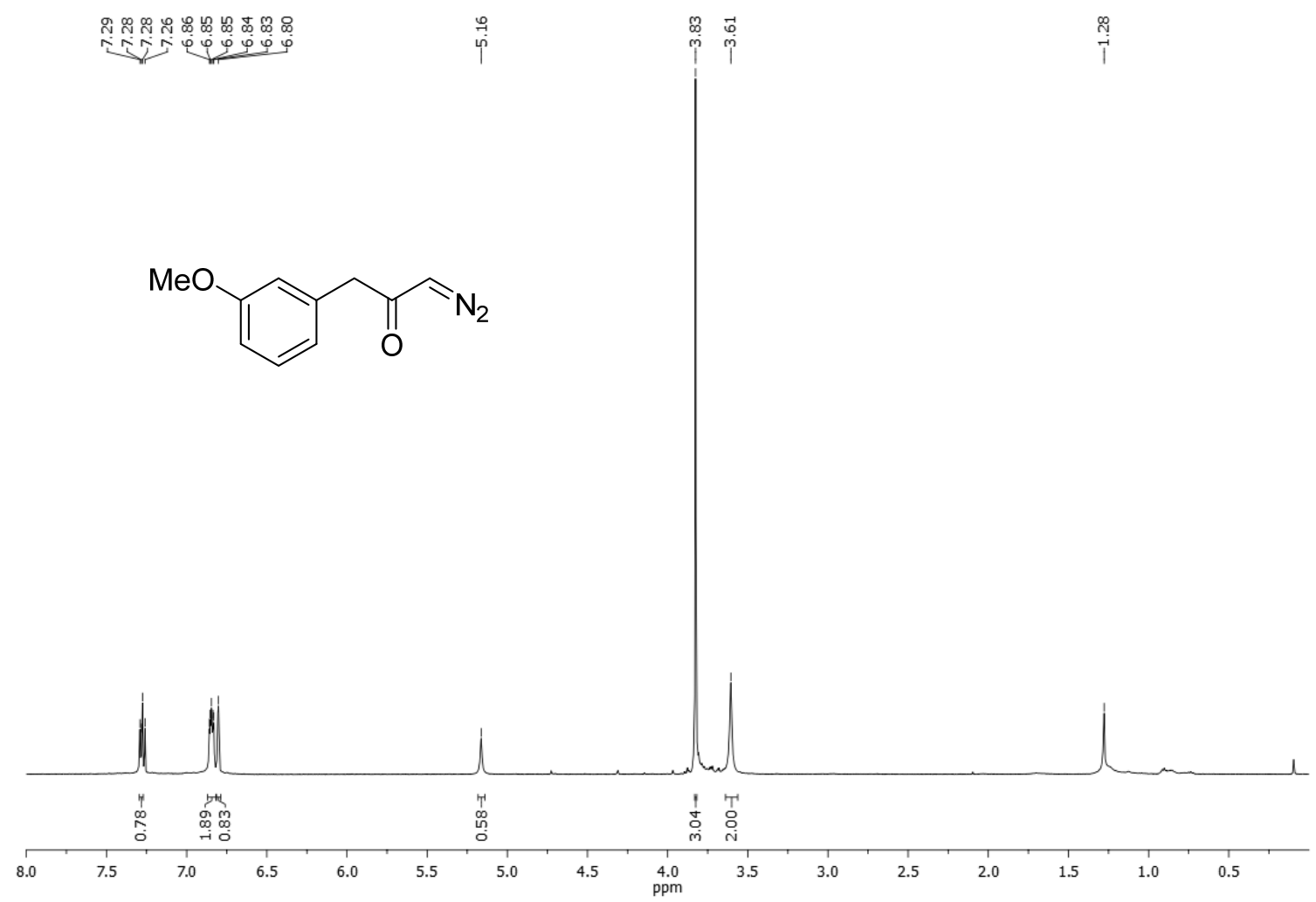

1-diazo-3-(3-methoxyphenyl)-2-propanone (6g), ${ }^{13} \mathrm{C} \mathrm{NMR}\left(126 \mathrm{MHz}, \mathrm{CDCl}_{3}\right)$ :

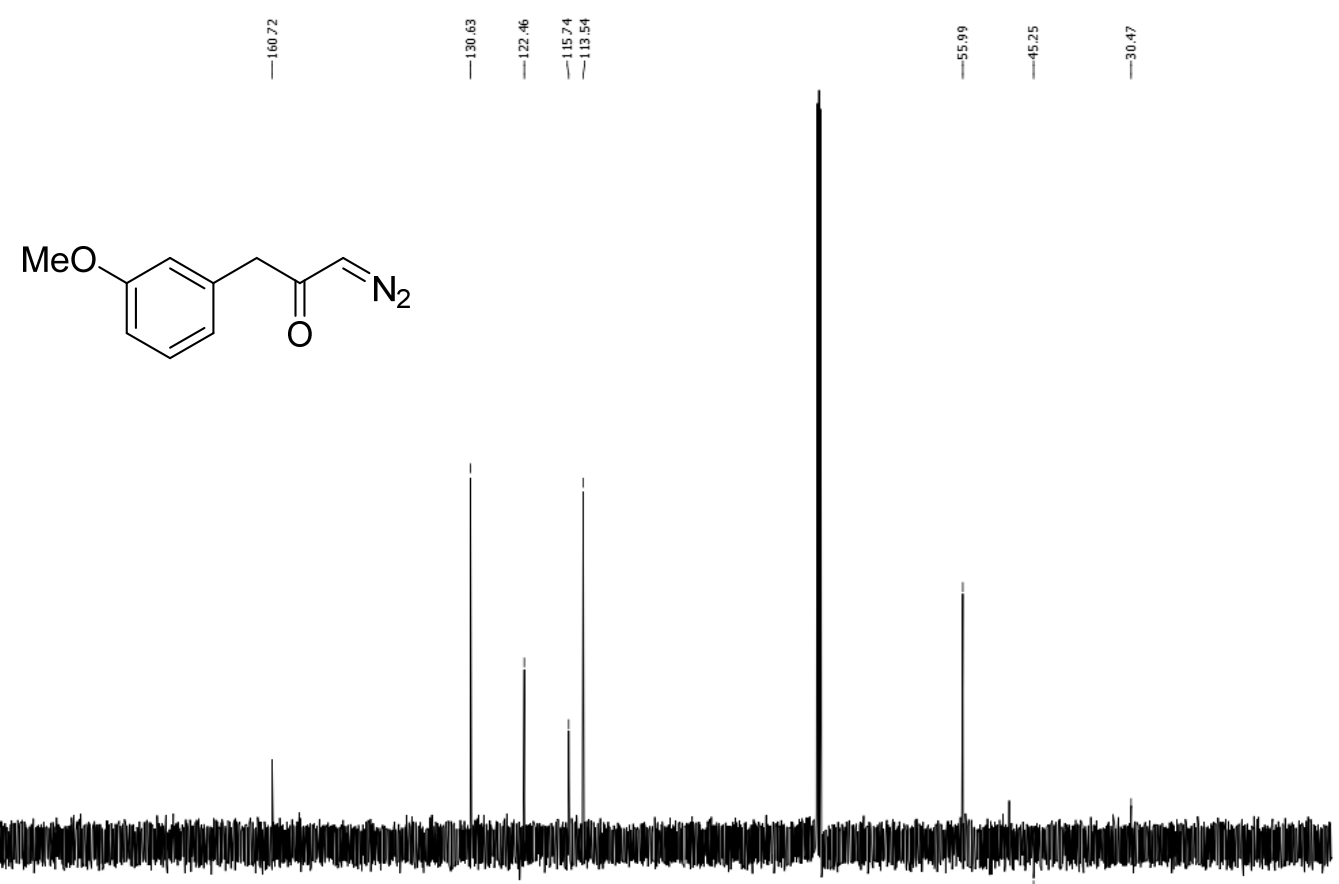

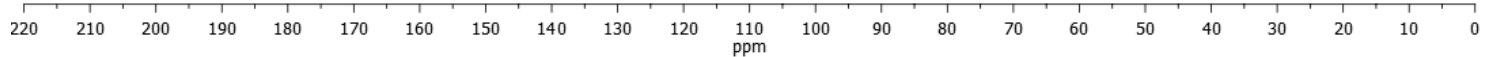


1-diazo-3-(3,4-(methylenedioxy)phenyl)-2-propanone (6h), ${ }^{1} \mathrm{H} \mathrm{NMR}\left(500 \mathrm{MHz}, \mathrm{CDCl}_{3}\right)$ :

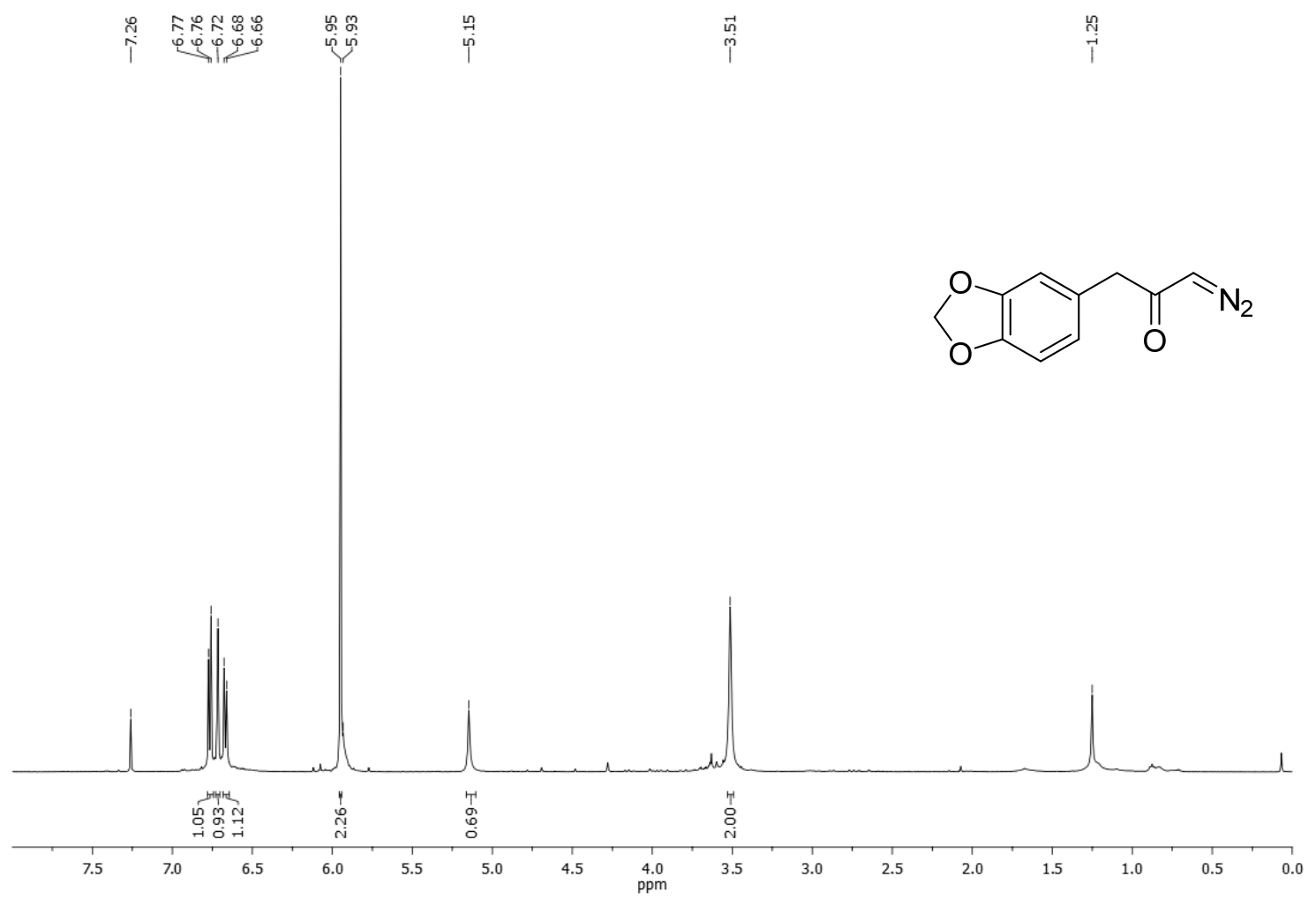

1-diazo-3-(3,4-(methylenedioxy)phenyl)-2-propanone (6h), ${ }^{13} \mathrm{C} \mathrm{NMR}\left(126 \mathrm{MHz}, \mathrm{CDCl}_{3}\right)$ :

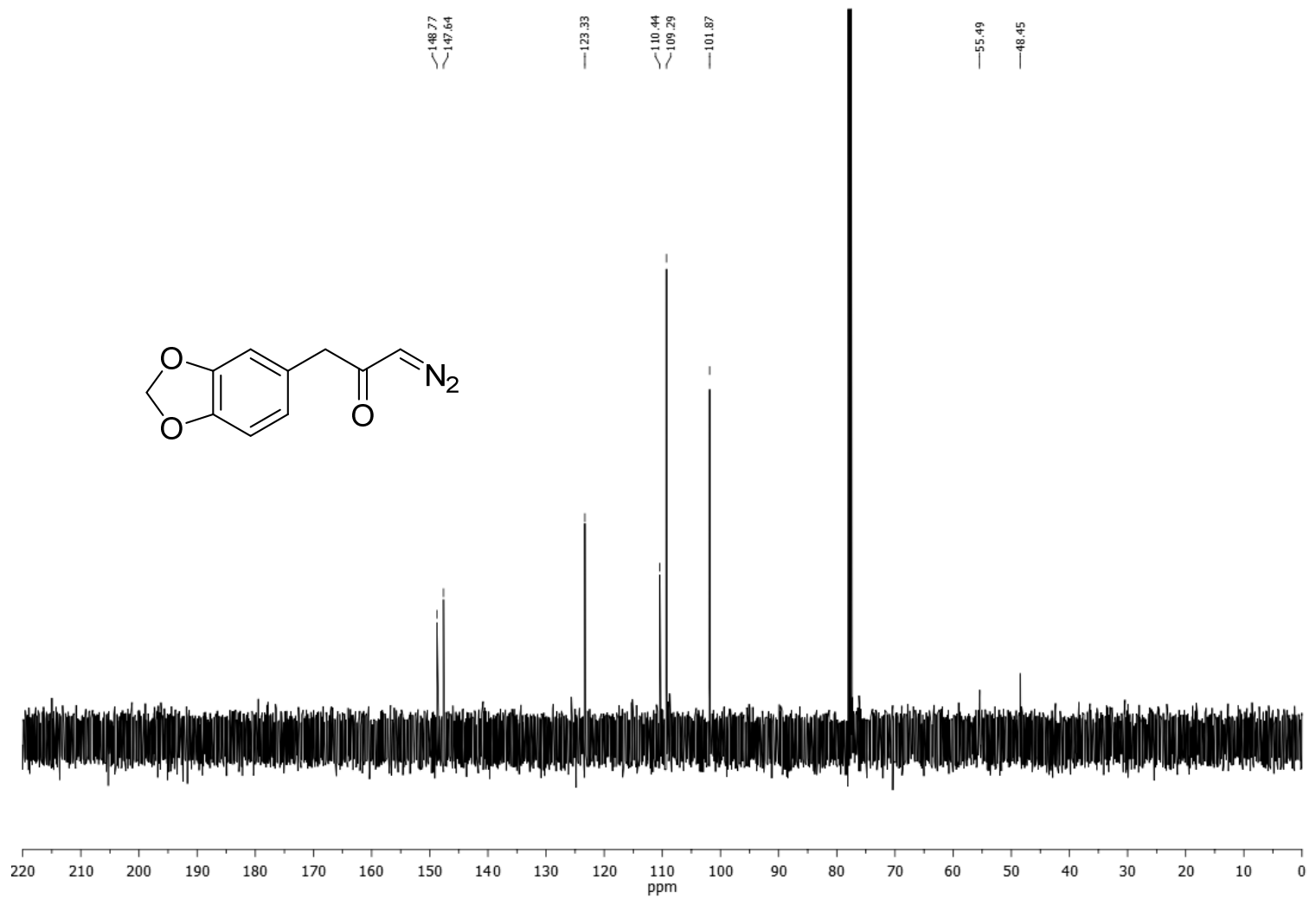


(S)-1-diazo-3-phenylbutan-2-one (6i), ${ }^{1} \mathrm{H}$ NMR (500 MHz, $\left.\mathrm{CDCl}_{3}\right)$ :

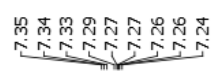

$\stackrel{\infty}{\varphi}$

i̊n.<smiles>CC(C=[W])c1ccccc1</smiles>
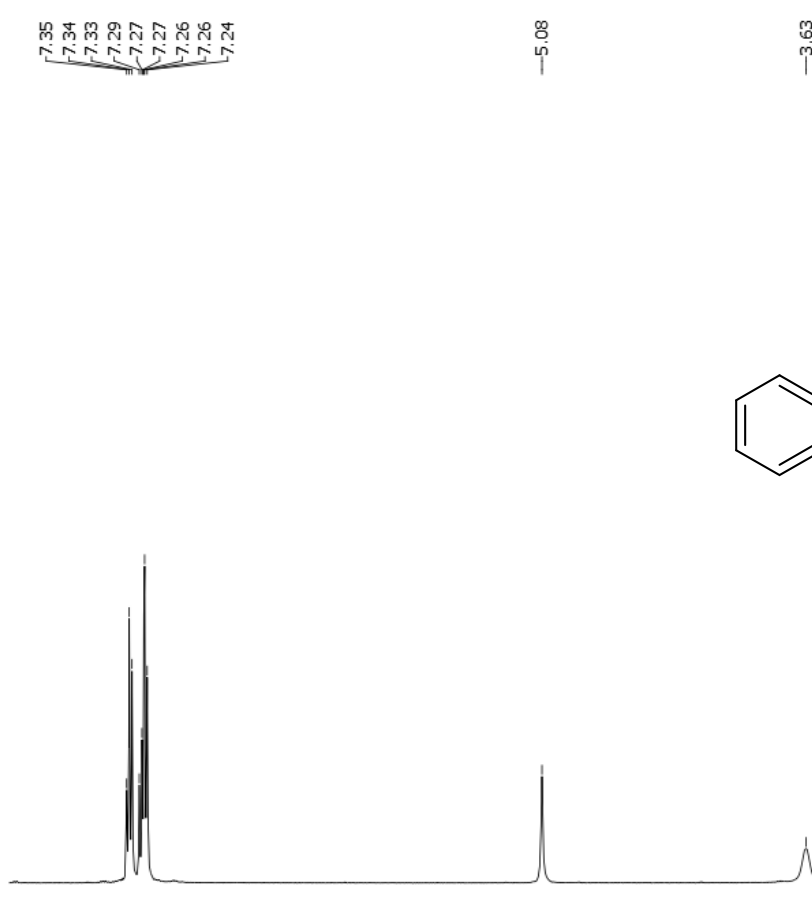

位

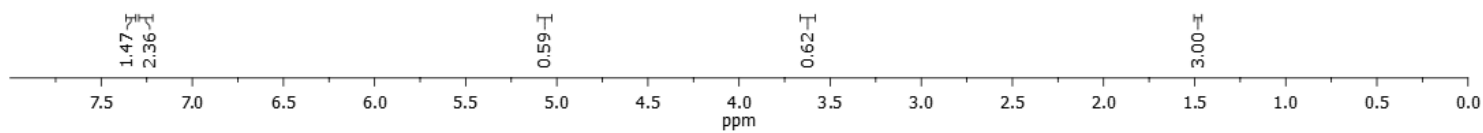

(S)-1-diazo-3-phenylbutan-2-one (6i), ${ }^{13} \mathrm{C}$ NMR (126 MHz, $\left.\mathrm{CDCl}_{3}\right)$ :

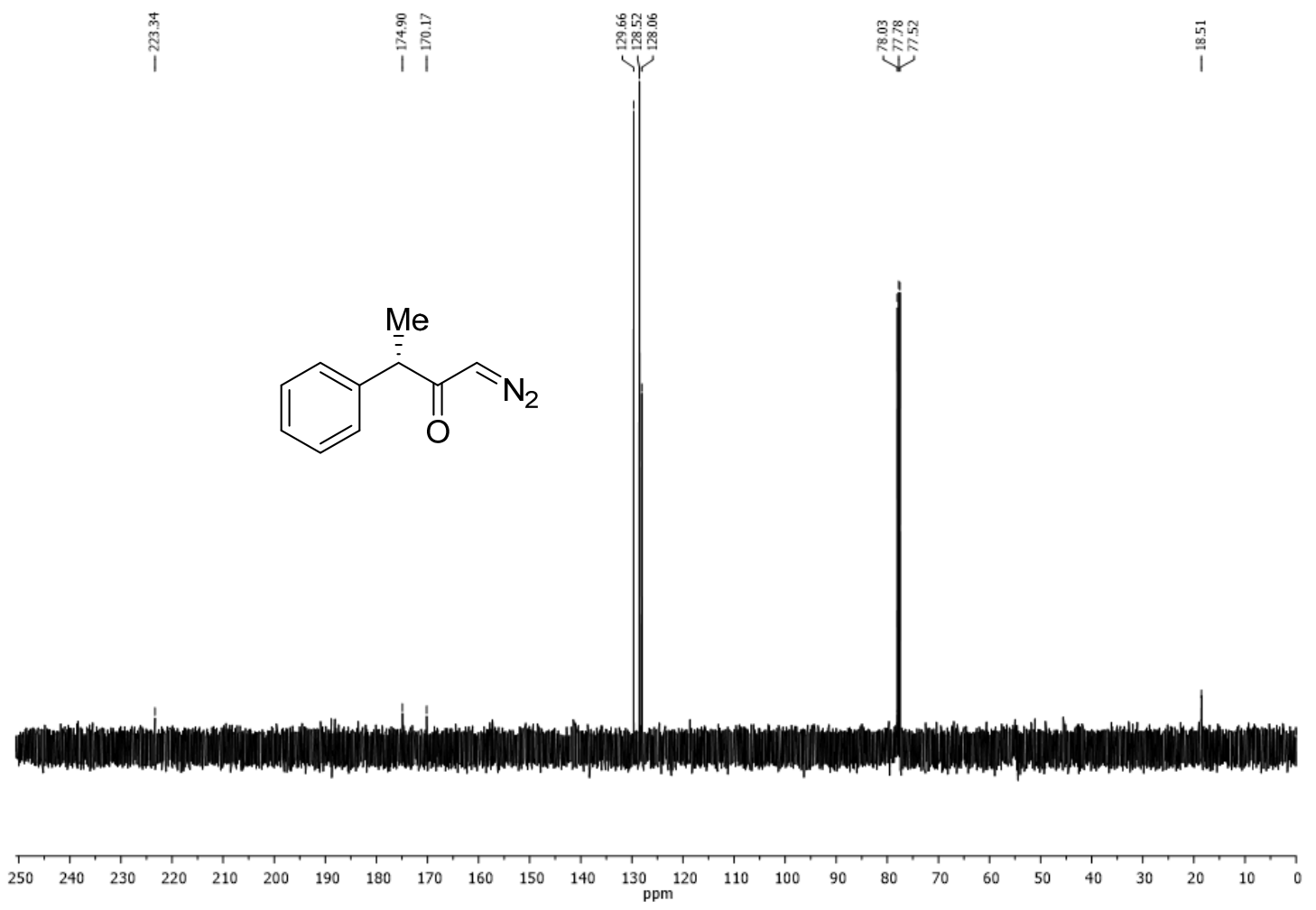


1-cyclohexyl-3-diazopropan-2-one (6k), ${ }^{1} \mathrm{H}$ NMR $\left(500 \mathrm{MHz}, \mathrm{CDCl}_{3}\right)$ :

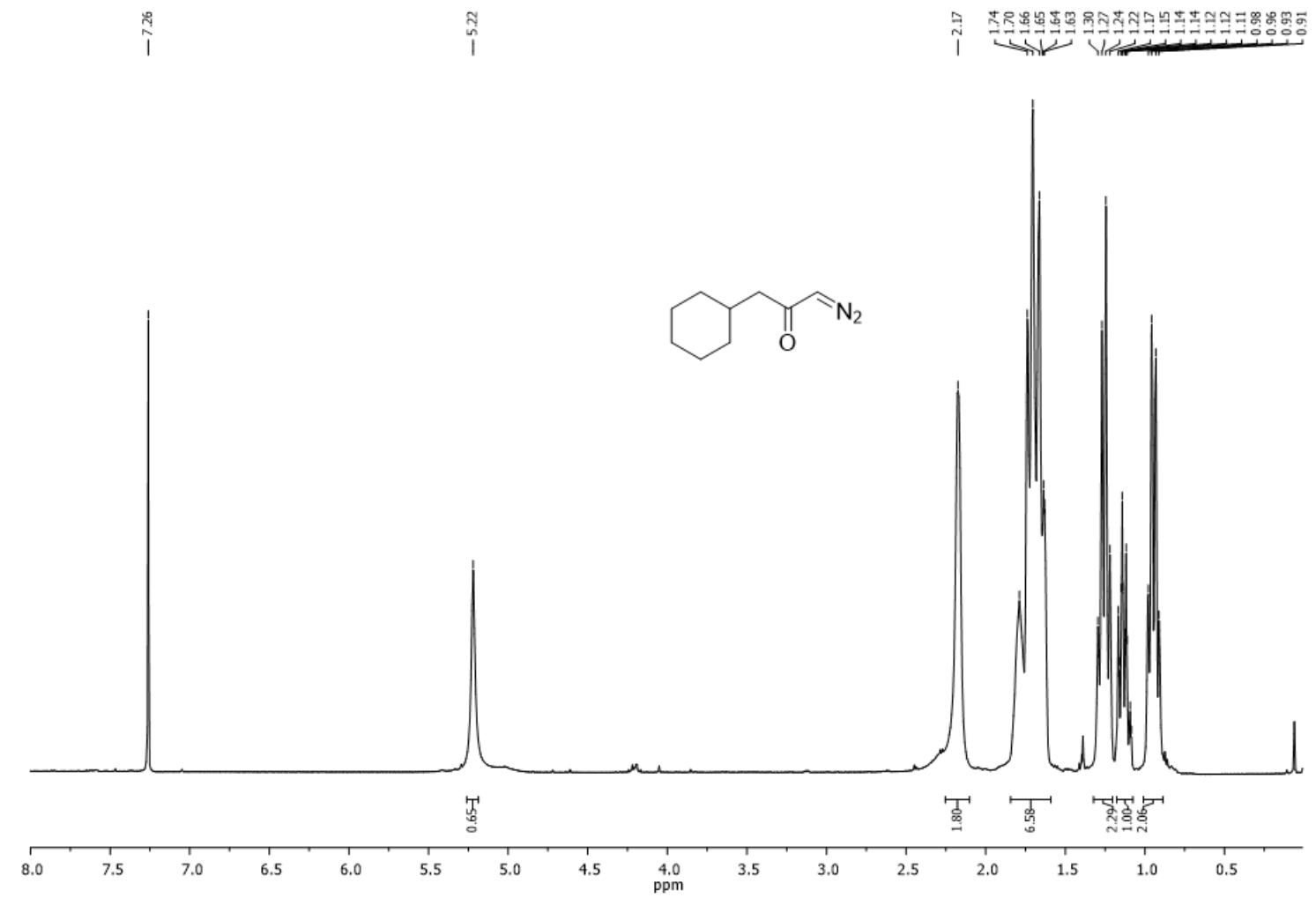

1-cyclohexyl-3-diazopropan-2-one (6k), ${ }^{13} \mathrm{C} \mathrm{NMR}\left(126 \mathrm{MHz}, \mathrm{CDCl}_{3}\right)$ :

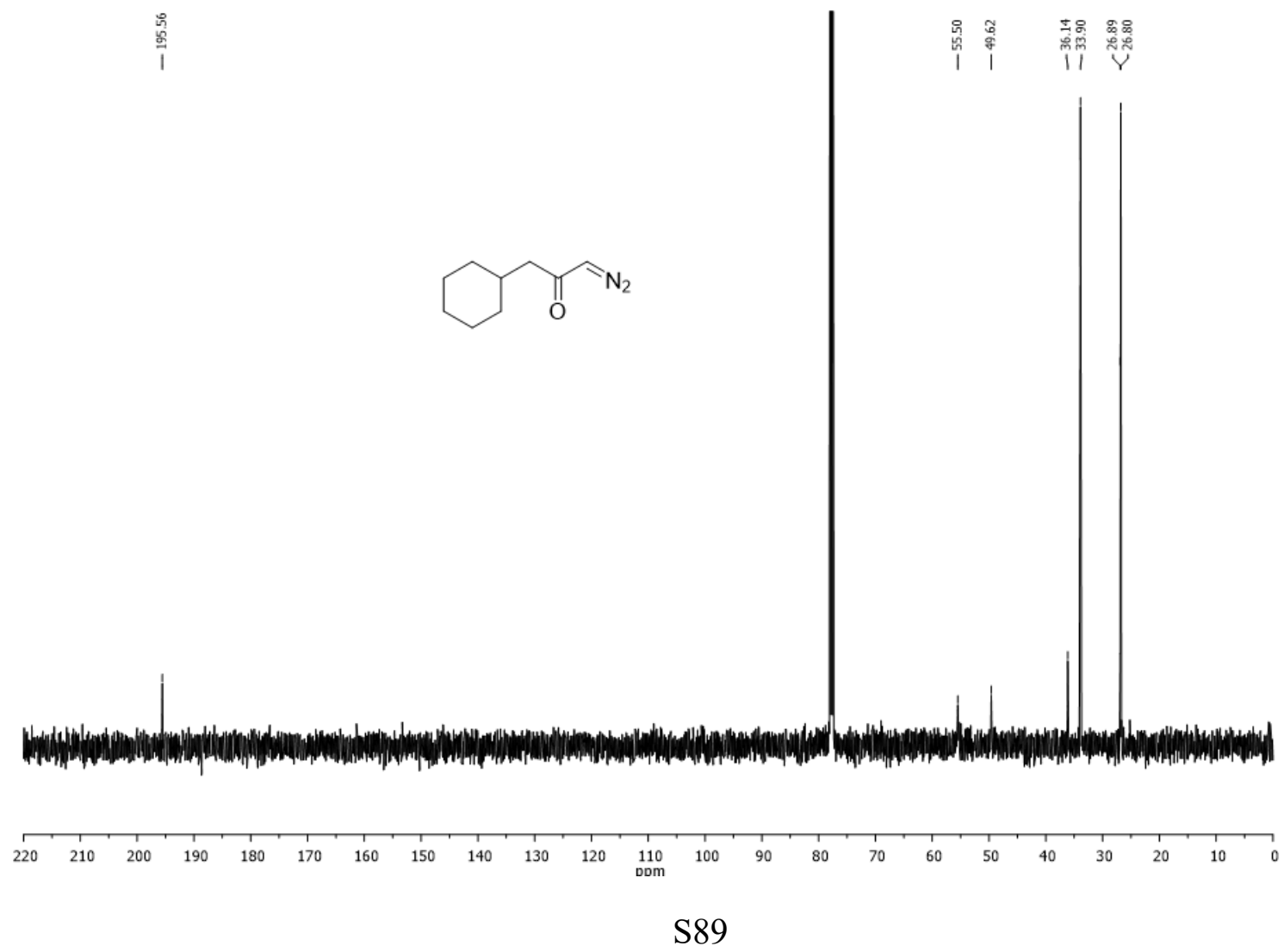


1-cyclopropyl-3-diazopropan-2-one (61), ${ }^{1} \mathrm{H}$ NMR $\left(500 \mathrm{MHz}, \mathrm{CDCl}_{3}\right)$ :

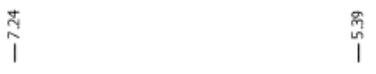

ฉุจ
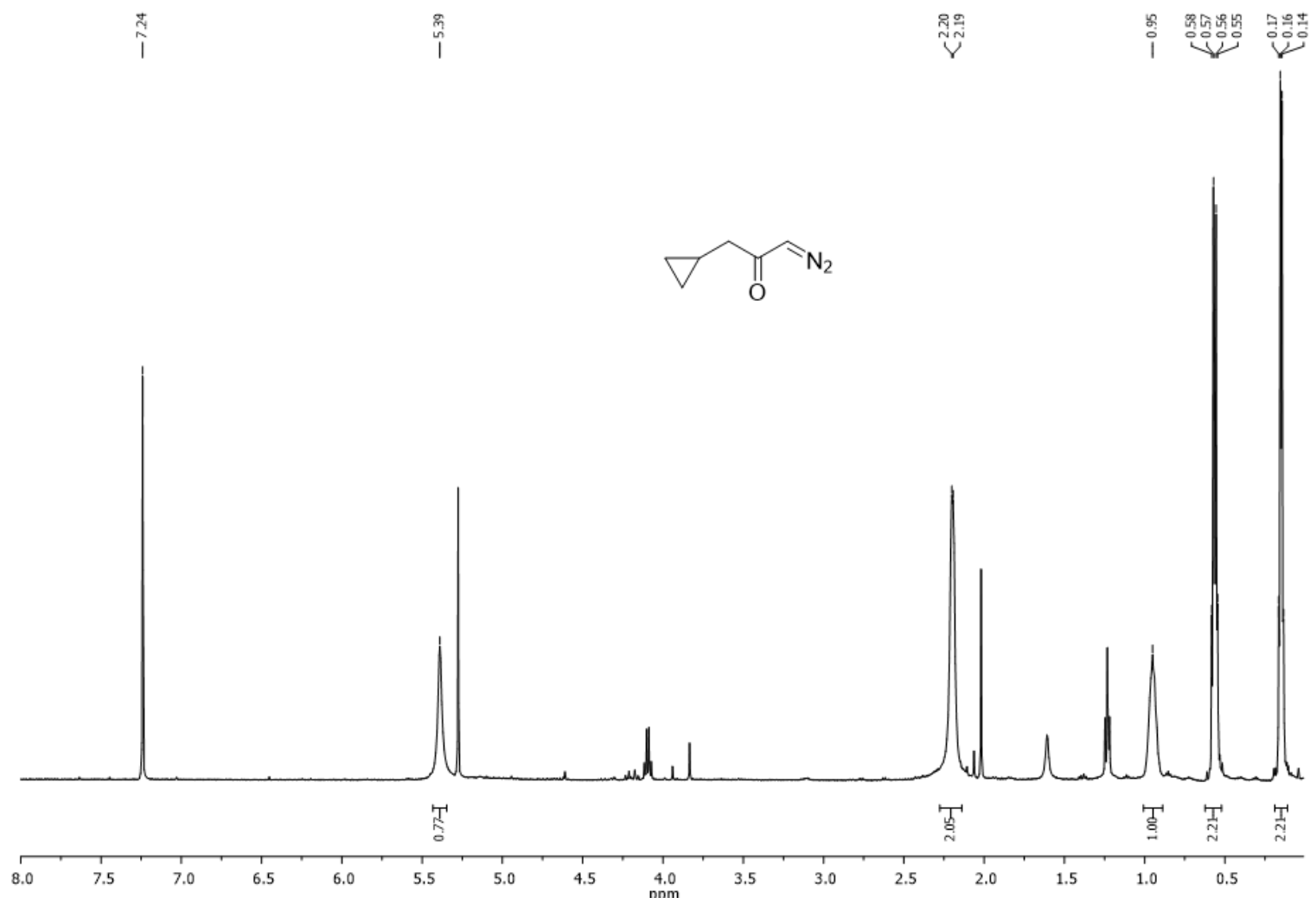

1-cyclopropyl-3-diazopropan-2-one (61), ${ }^{13} \mathrm{C} \mathrm{NMR}\left(126 \mathrm{MHz}, \mathrm{CDCl}_{3}\right)$ :

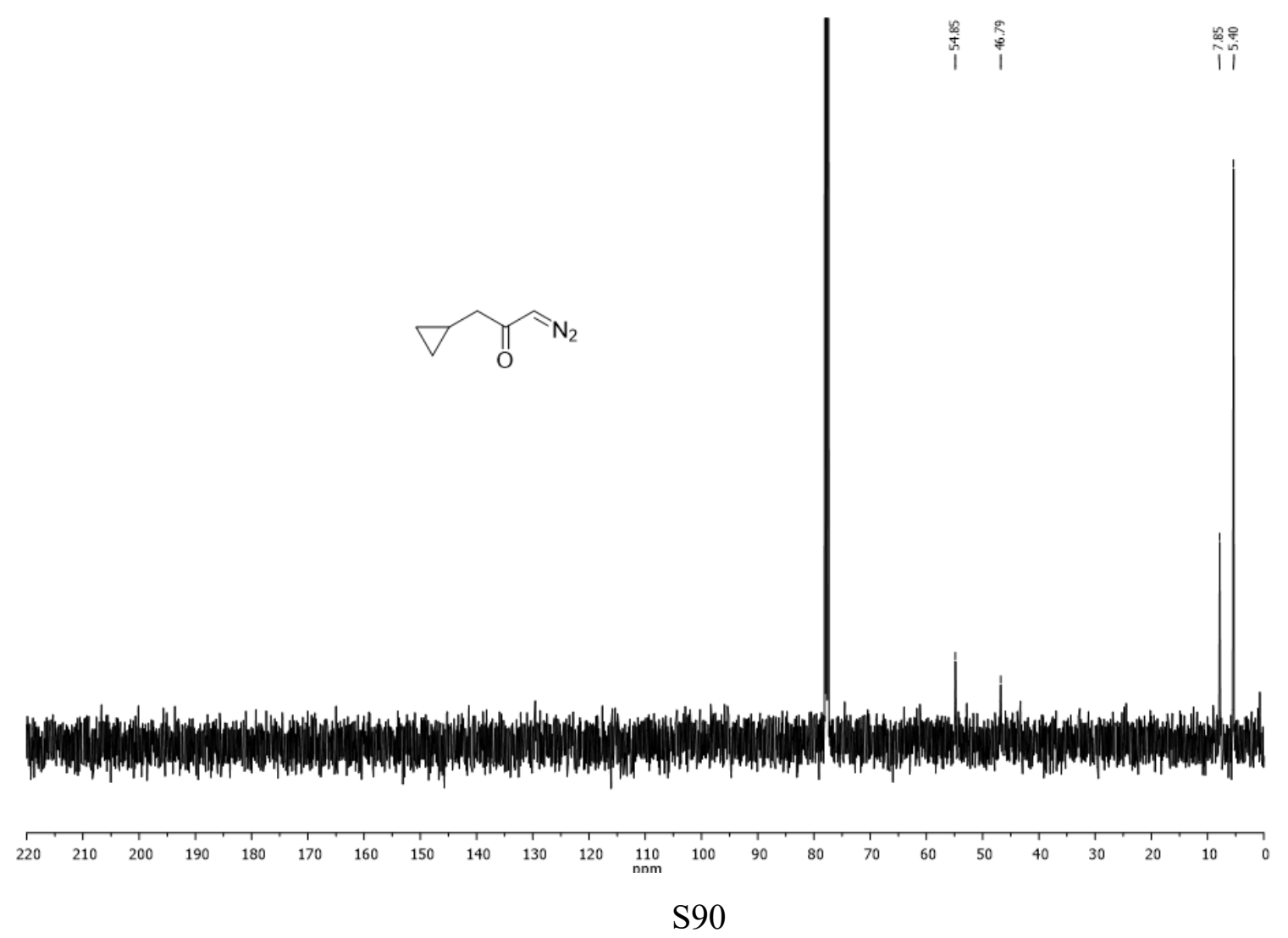


1-diazo-5-methylhexan-2-one (6m), ${ }^{1} \mathrm{H}$ NMR (500 MHz, $\left.\mathrm{CDCl}_{3}\right)$ :

สู
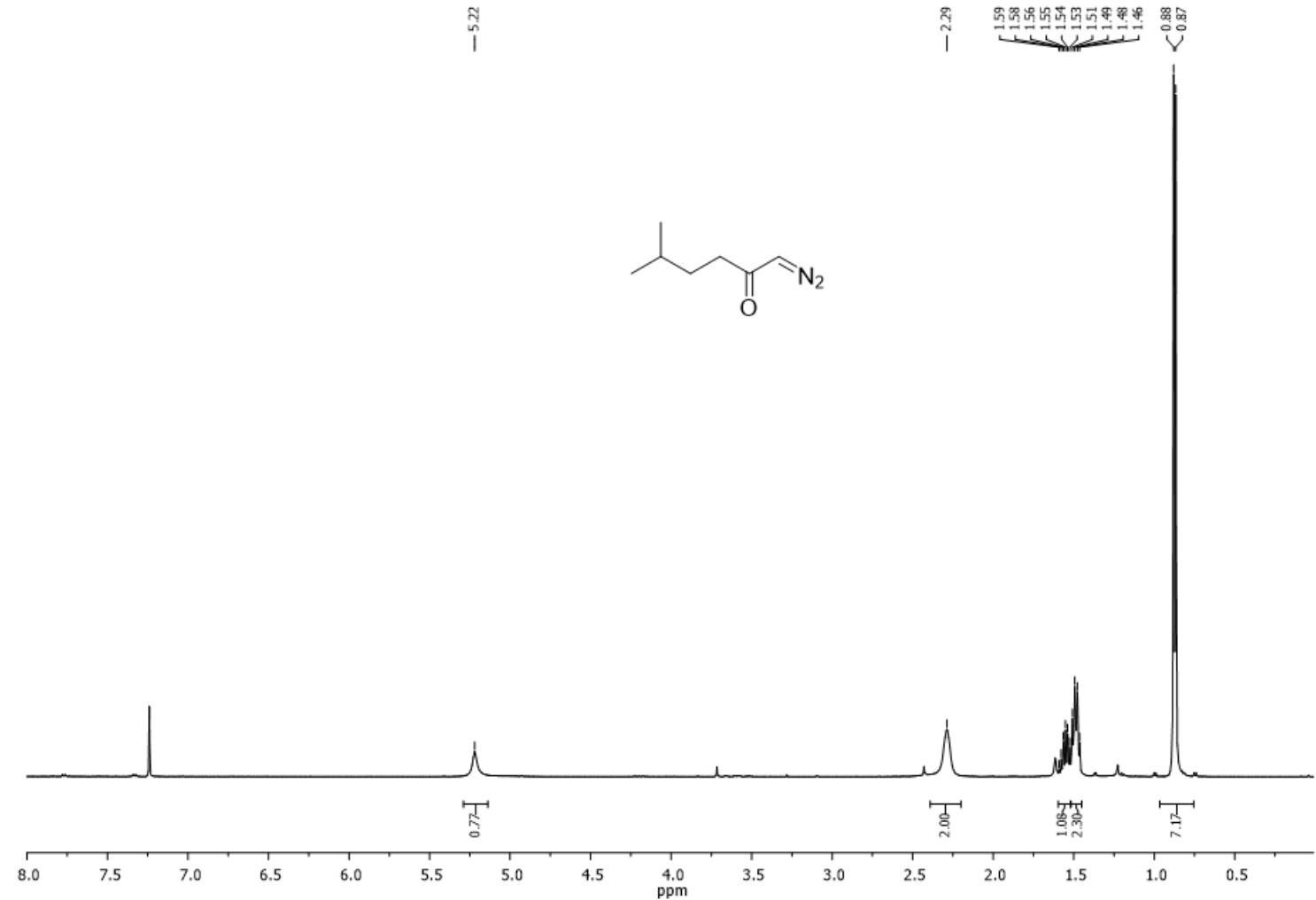

1-diazo-5-methylhexan-2-one (6m), ${ }^{13} \mathrm{C} \mathrm{NMR}\left(126 \mathrm{MHz}, \mathrm{CDCl}_{3}\right)$ :

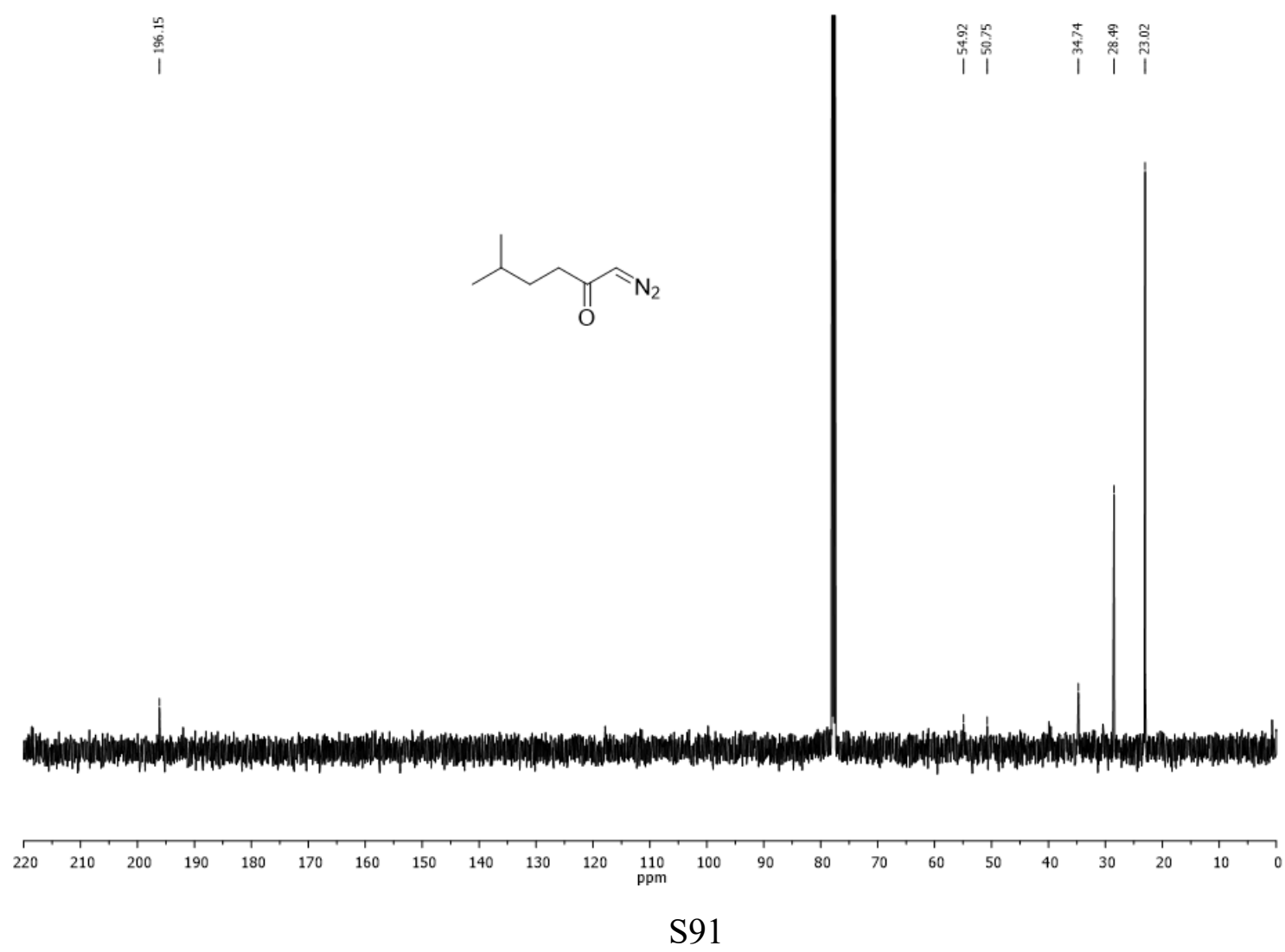


1-diazo-4-methylpentan-2-one (6n), ${ }^{1} \mathrm{H} \mathrm{NMR}\left(500 \mathrm{MHz}, \mathrm{CDCl}_{3}\right)$ :
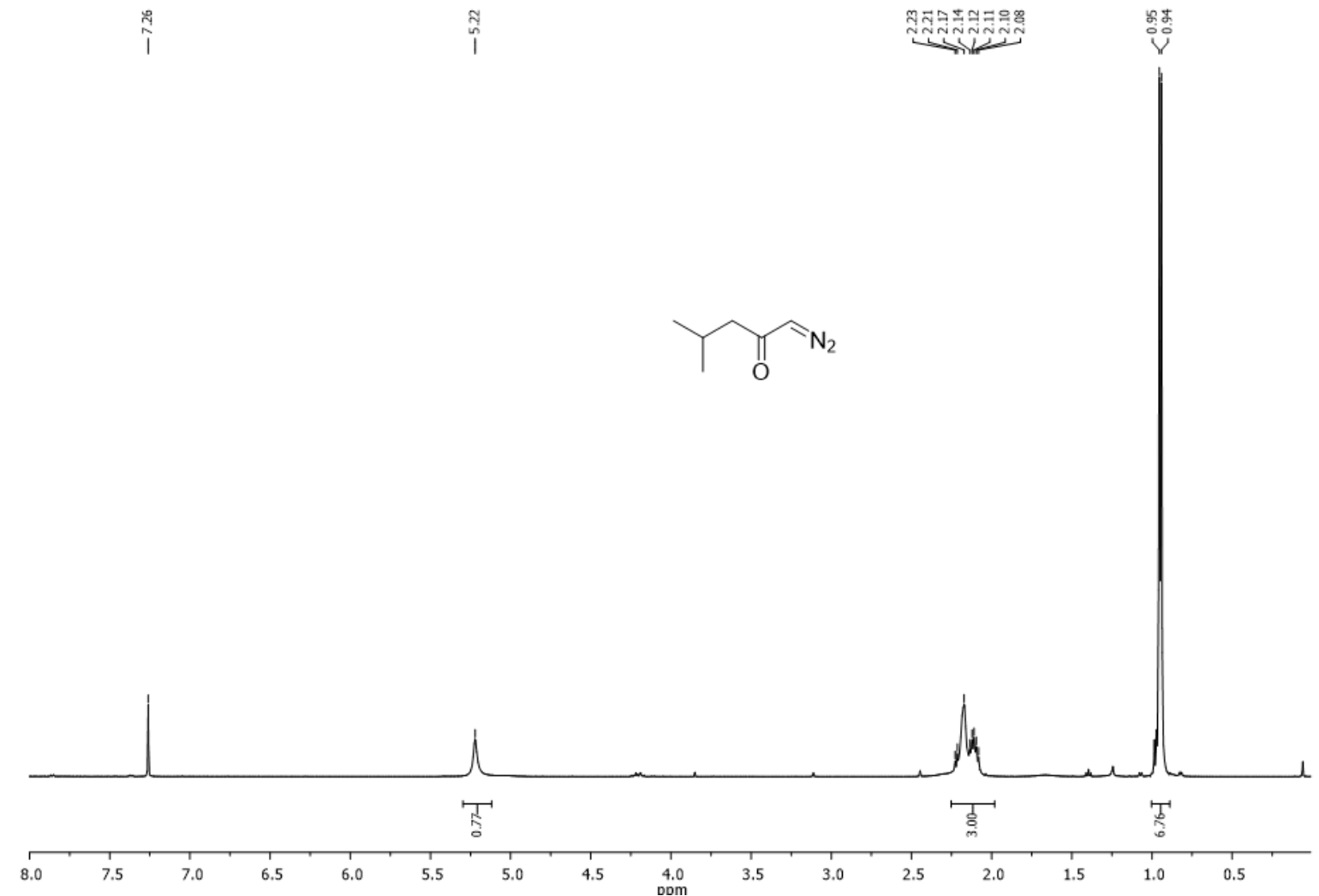

1-diazo-4-methylpentan-2-one (6n), $\left.{ }^{13} \mathrm{C} \mathrm{NMR} \mathrm{(126} \mathrm{MHz,} \mathrm{CDCl}_{3}\right)$ :
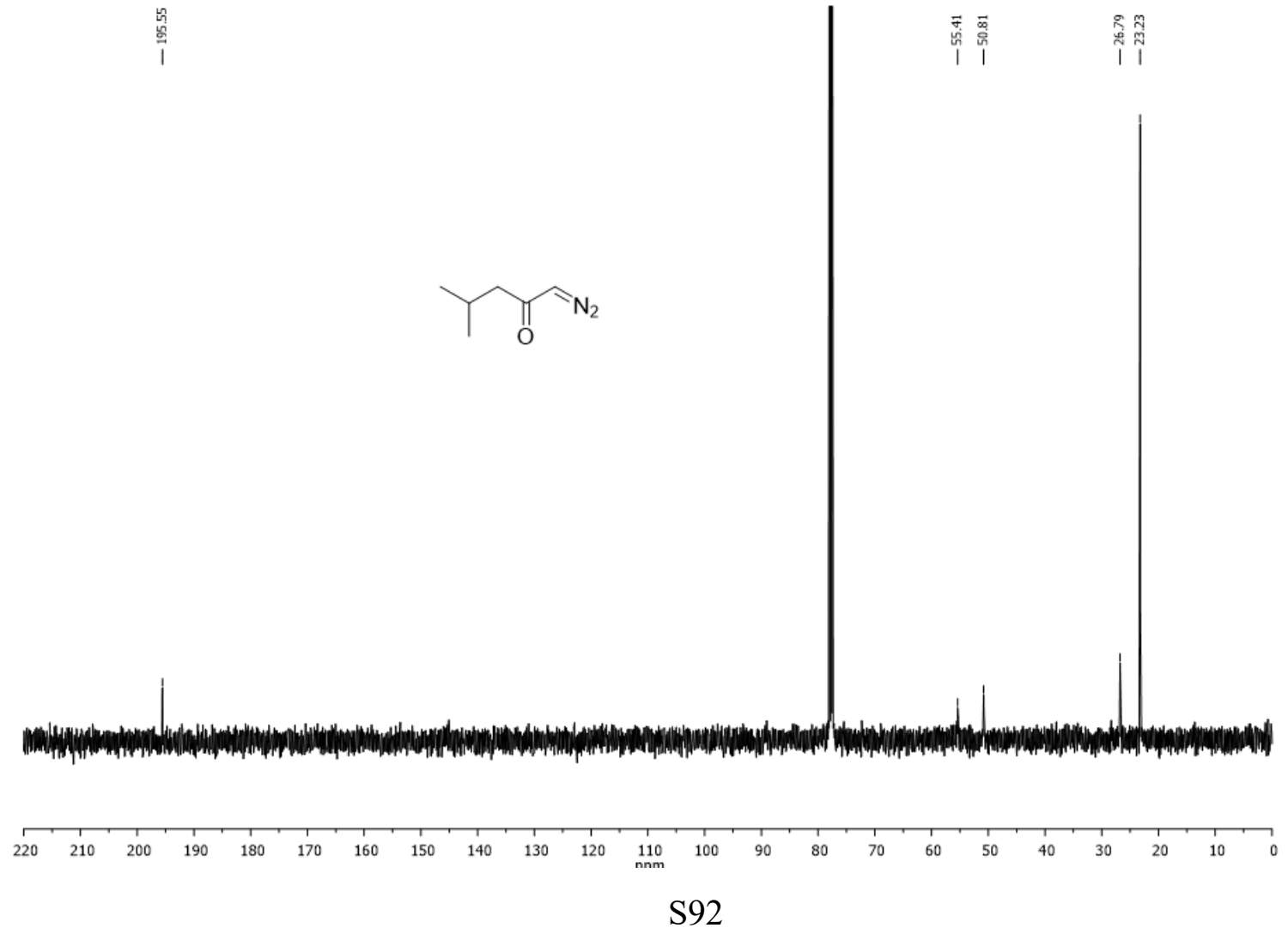
2-phenyl-1-((1S, 2S)-2-phenylcyclopropyl)ethan-1-one (3), ${ }^{1} \mathrm{H} \mathrm{NMR}\left(500 \mathrm{MHz}, \mathrm{CDCl}_{3}\right)$ :
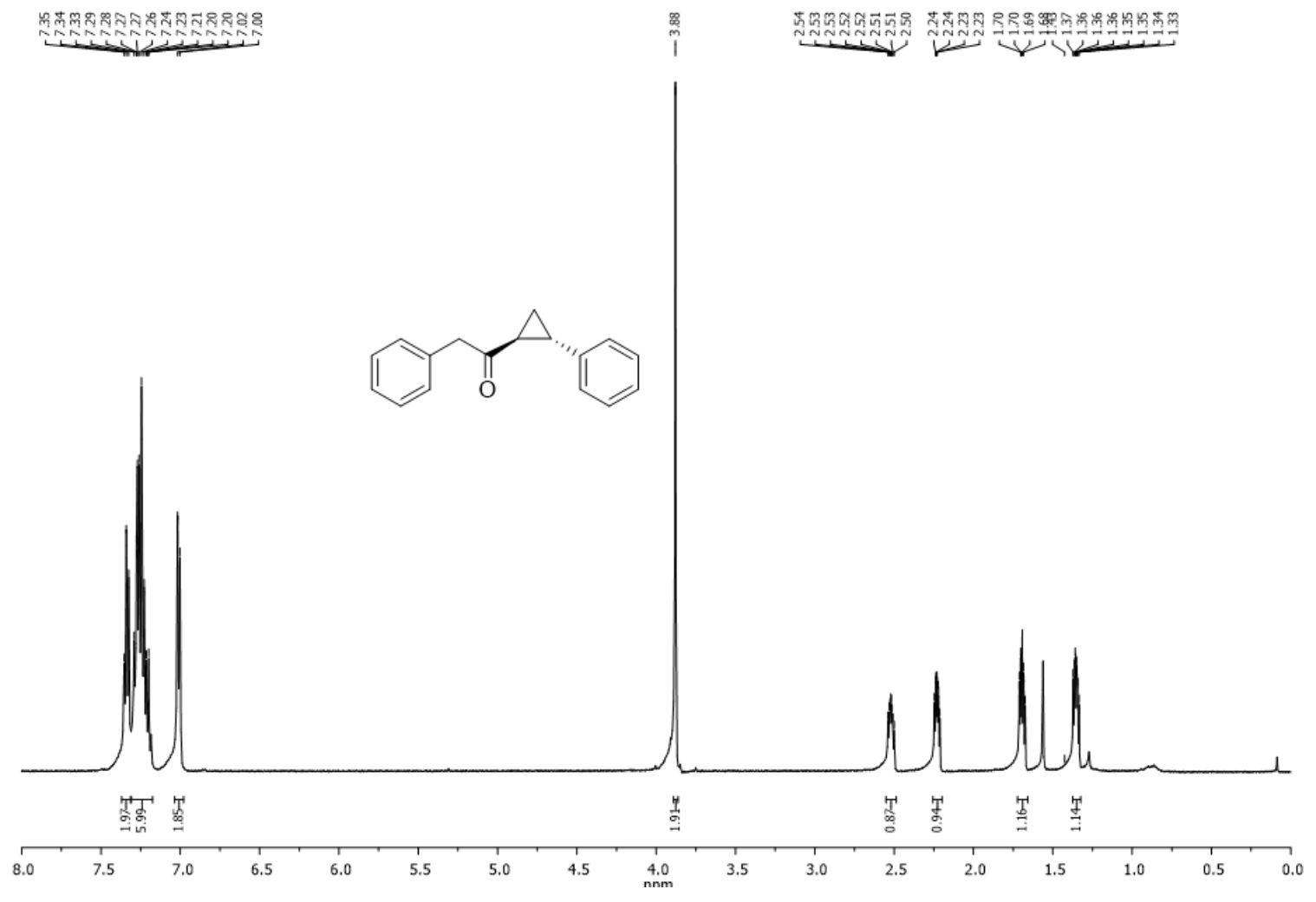

2-phenyl-1-((1S, 2S)-2-phenylcyclopropyl)ethan-1-one (3), ${ }^{13} \mathrm{C} \mathrm{NMR}\left(126 \mathrm{MHz}, \mathrm{CDCl}_{3}\right)$ :

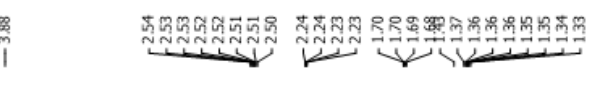

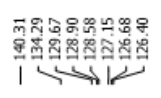

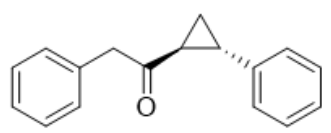


2-phenyl-1-((1S, 2S)-2-(p-tolyl)cyclopropyl)ethan-1-one (5a), ${ }^{1} \mathrm{H}$ NMR (500 MHz, $\left.\mathrm{CDCl}_{3}\right)$ :

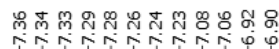

م.

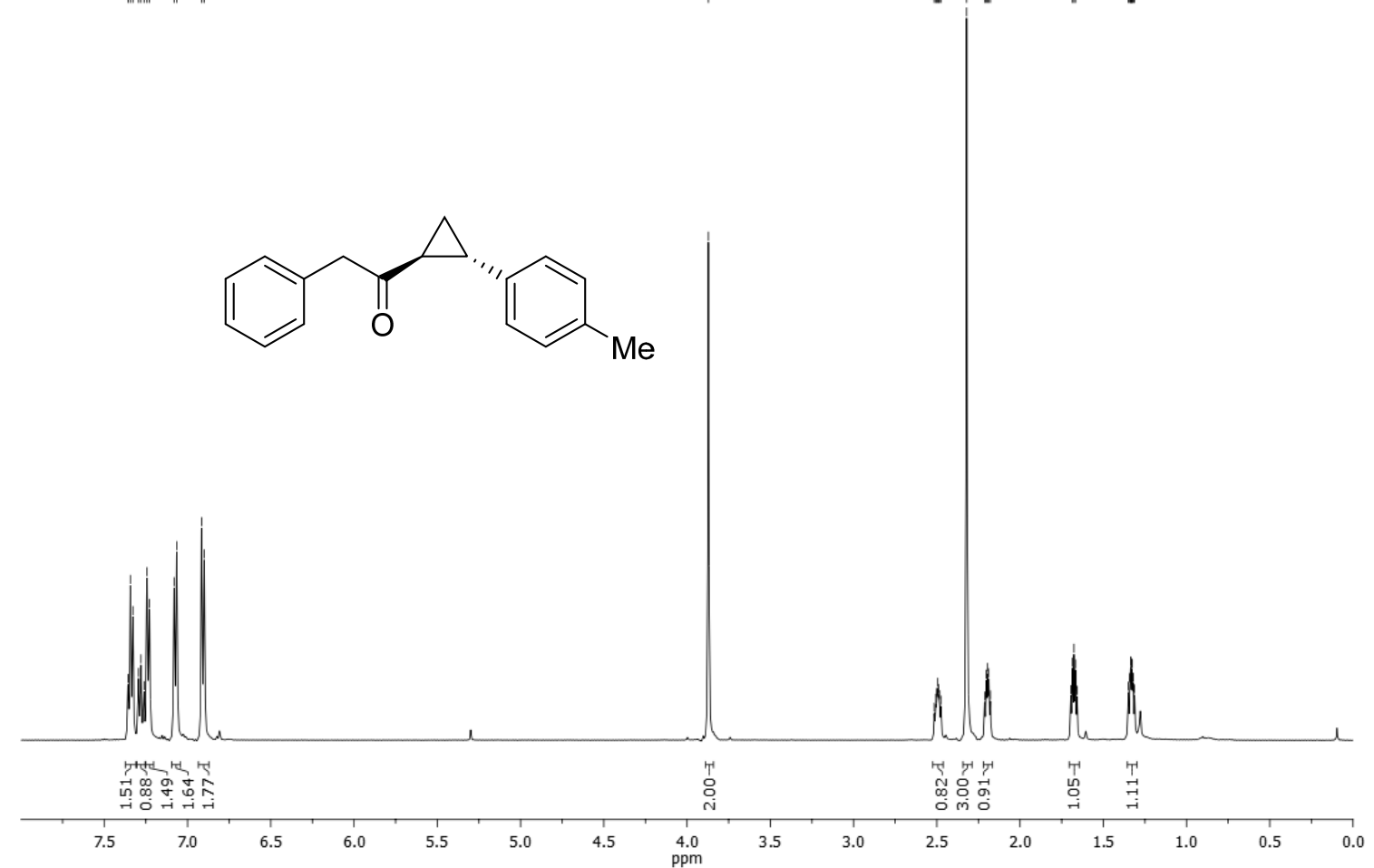

2-phenyl-1-((1S, 2S)-2-(p-tolyl)cyclopropyl)ethan-1-one (5a), ${ }^{13} \mathrm{C}$ NMR (126 MHz, $\left.\mathrm{CDCl}_{3}\right)$ :

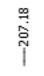

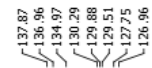

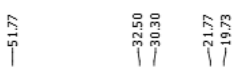<smiles>Cc1ccc([C@@H]2C[C@H]2C(=O)Cc2ccccc2)cc1</smiles>

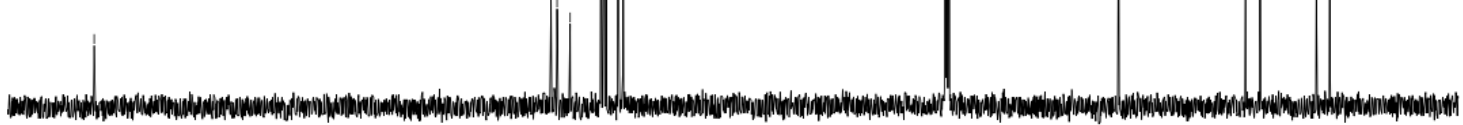

$\begin{array}{lllllllllll}220 & 210 & 200 & 190 & 180 & 170 & 160 & 150 & 140 & 130 & 120\end{array}$ 
1-((1S, 2S)-2-(4-methoxyphenyl)cyclopropyl)-2-phenylethan-1-one (5b), ${ }^{1} \mathrm{H}$ NMR (500 MHz, $\left.\mathrm{CDCl}_{3}\right)$ :

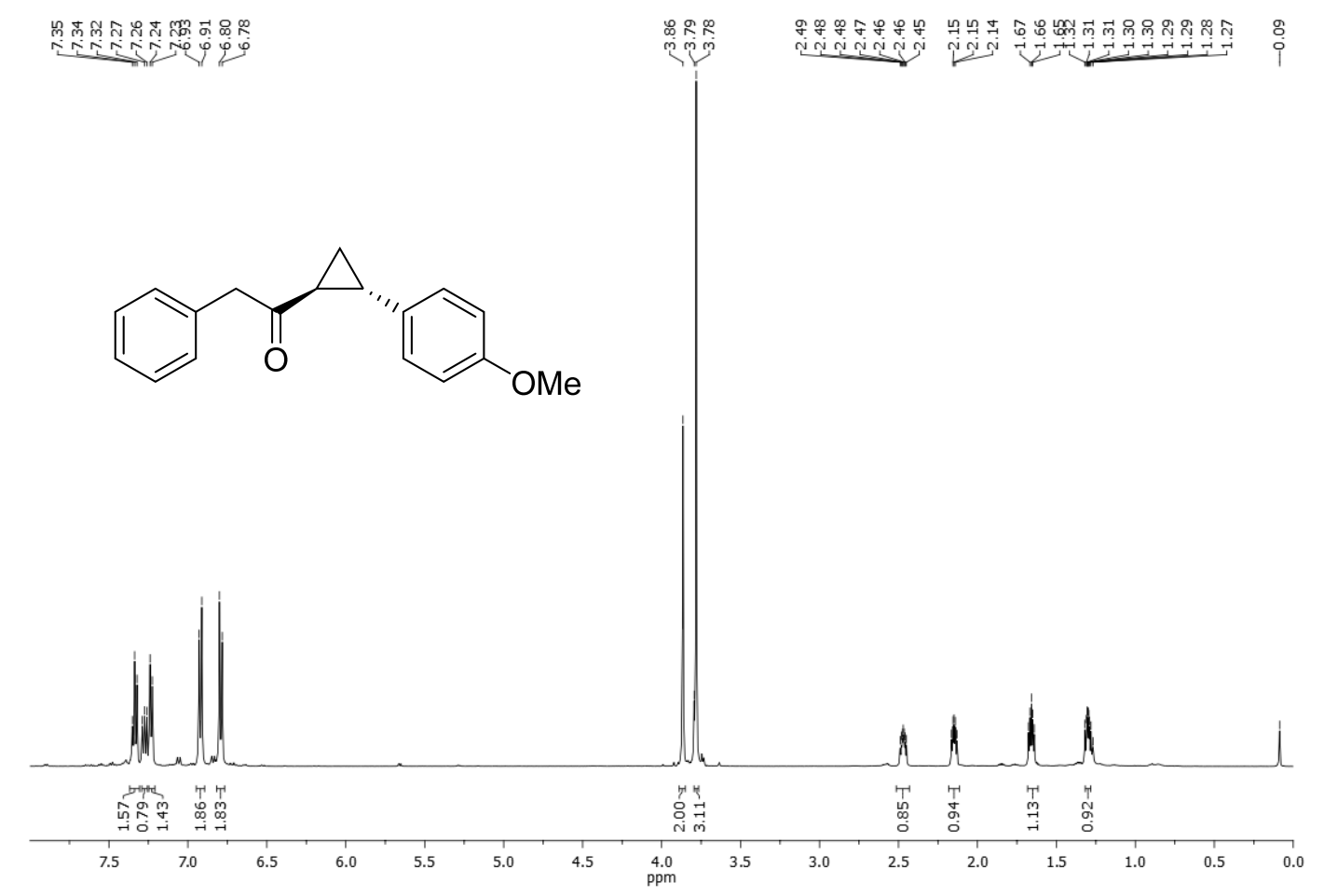

1-((1S, 2S)-2-(4-methoxyphenyl)cyclopropyl)-2-phenylethan-1-one (5b), ${ }^{13} \mathrm{C}$ NMR (126 MHz, $\left.\mathrm{CDCl}_{3}\right)$ :
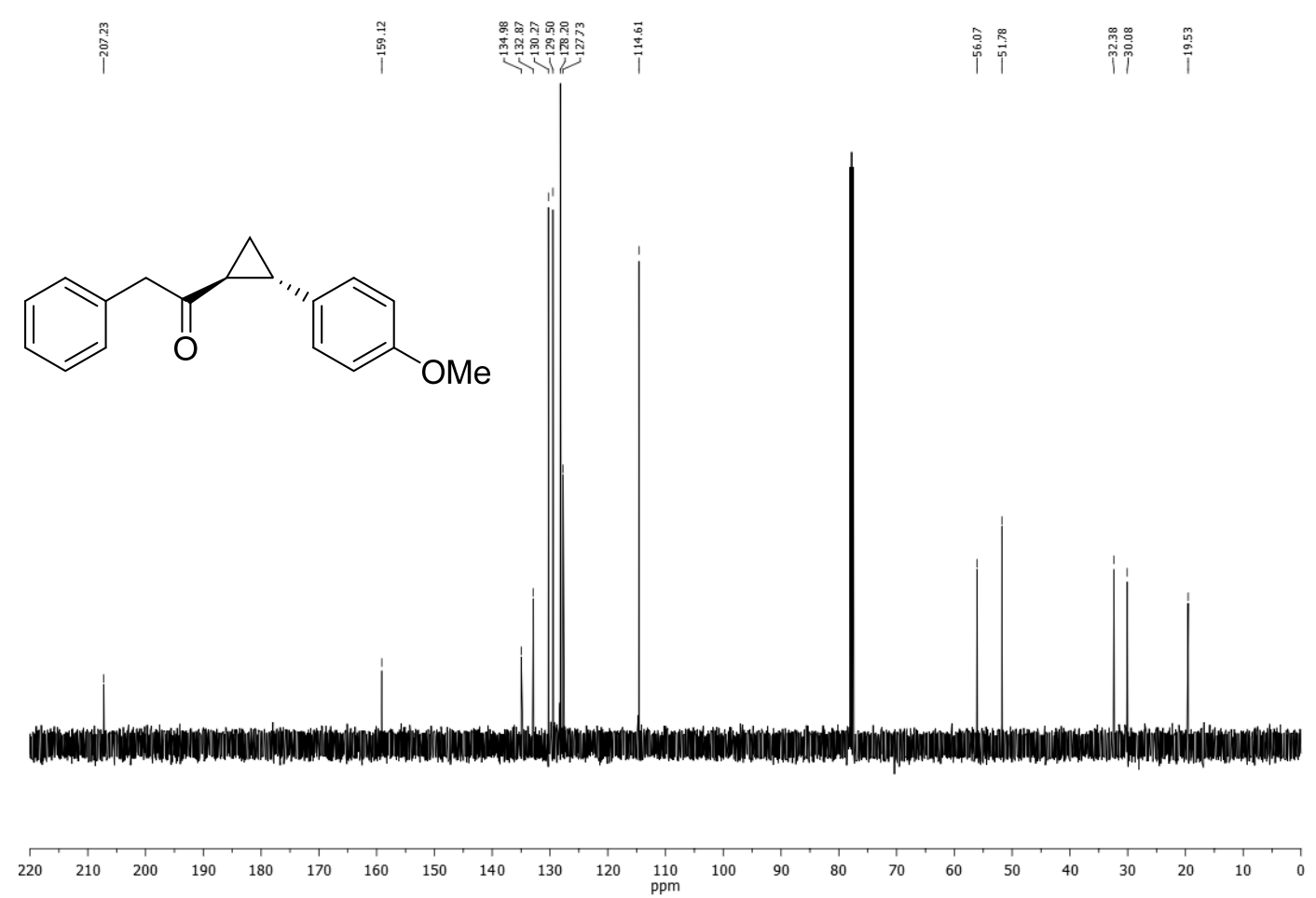
1-((1S, 2S)-2-(4-chlorophenyl)cyclopropyl)-2-phenylethan-1-one (5c), ${ }^{1} \mathrm{H}$ NMR (500 MHz, $\left.\mathrm{CDCl}_{3}\right)$ :

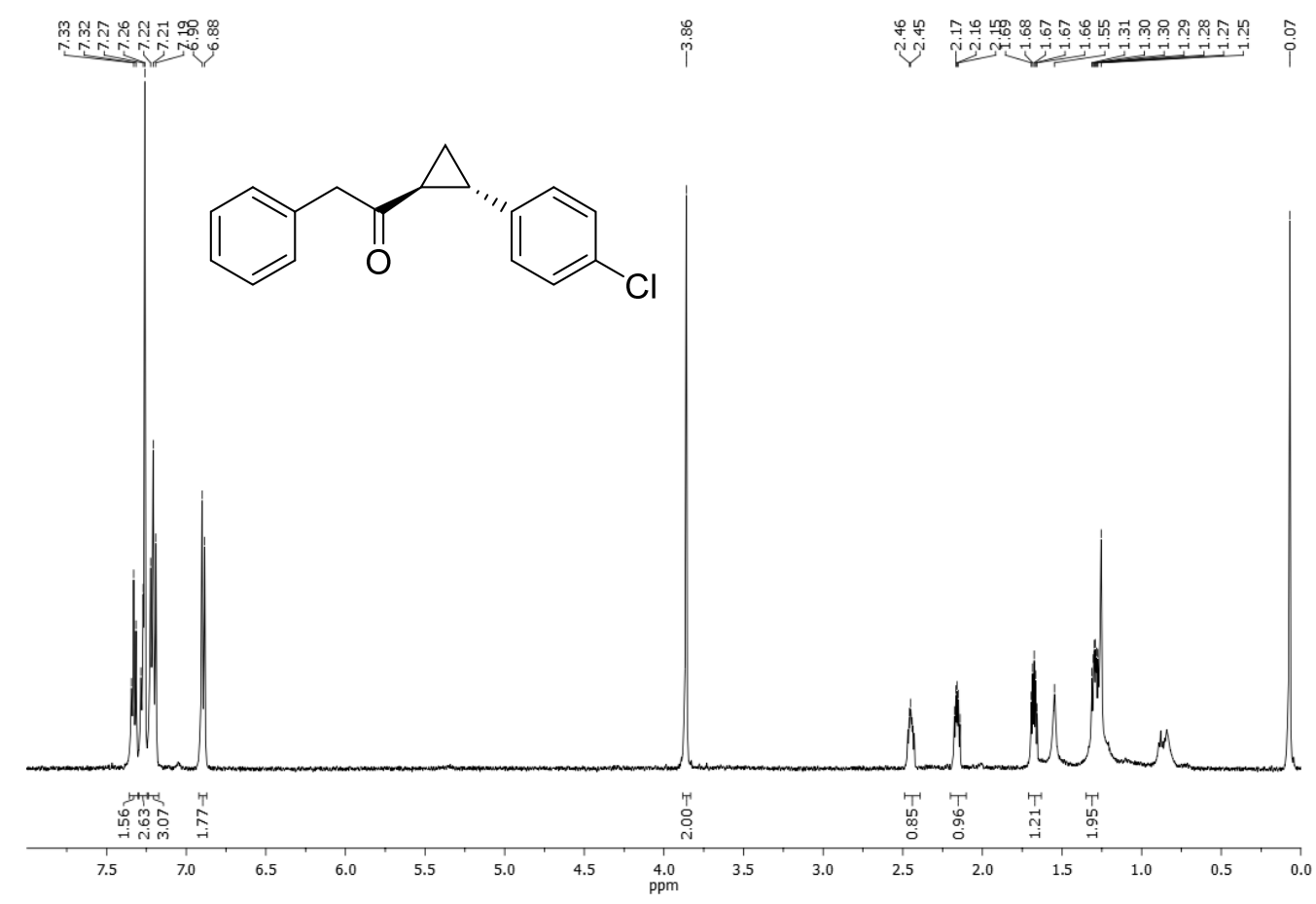

1-((1S, 2S)-2-(4-chlorophenyl)cyclopropyl)-2-phenylethan-1-one (5a), ${ }^{13} \mathrm{C}$ NMR (126 MHz, $\left.\mathrm{CDCl}_{3}\right)$ :
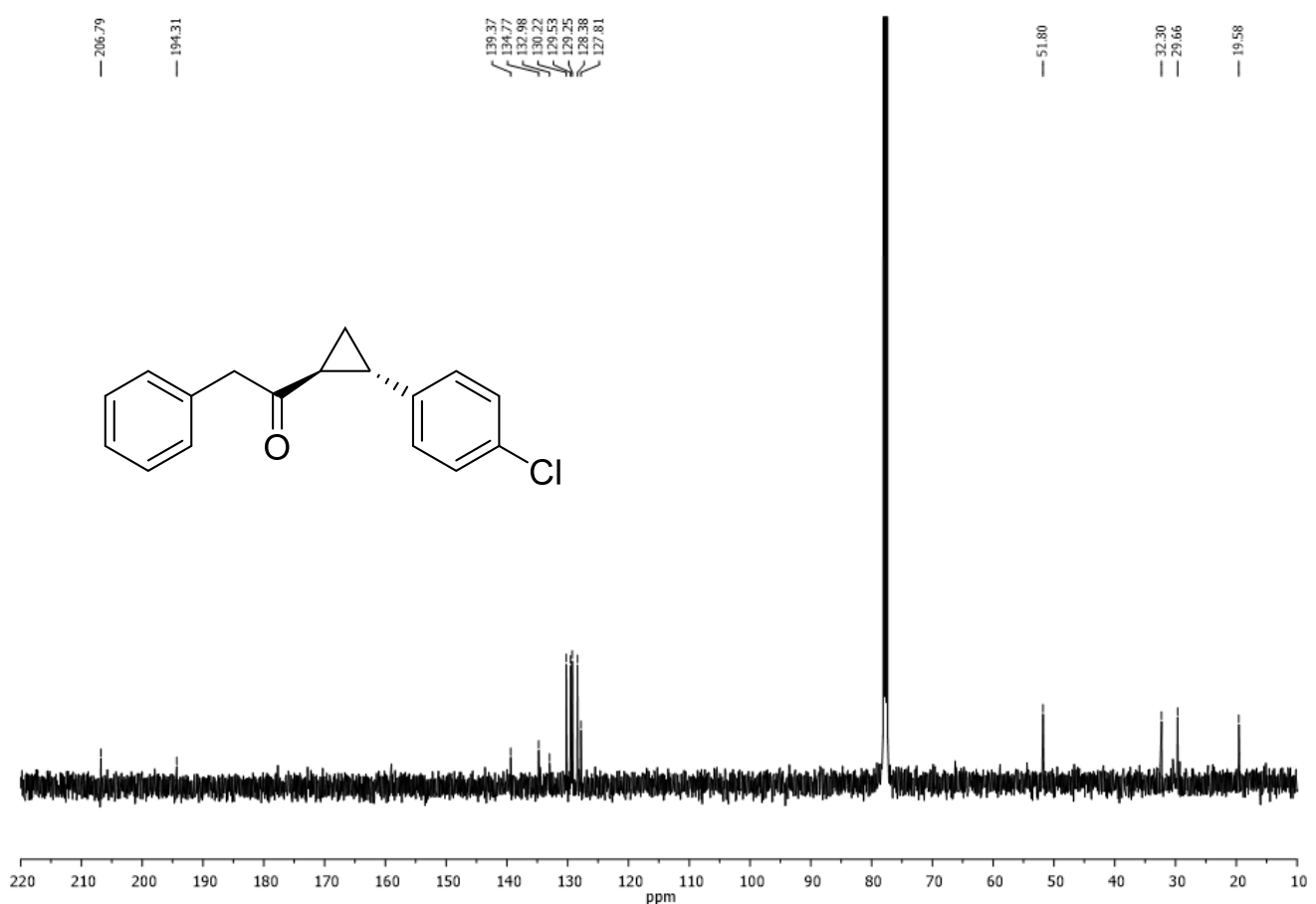
1-((1S, 2S)-2-(4-bromophenyl)cyclopropyl)-2-phenylethan-1-one (5d), ${ }^{1} \mathrm{H}$ NMR (500 MHz, $\left.\mathrm{CDCl}_{3}\right)$ :

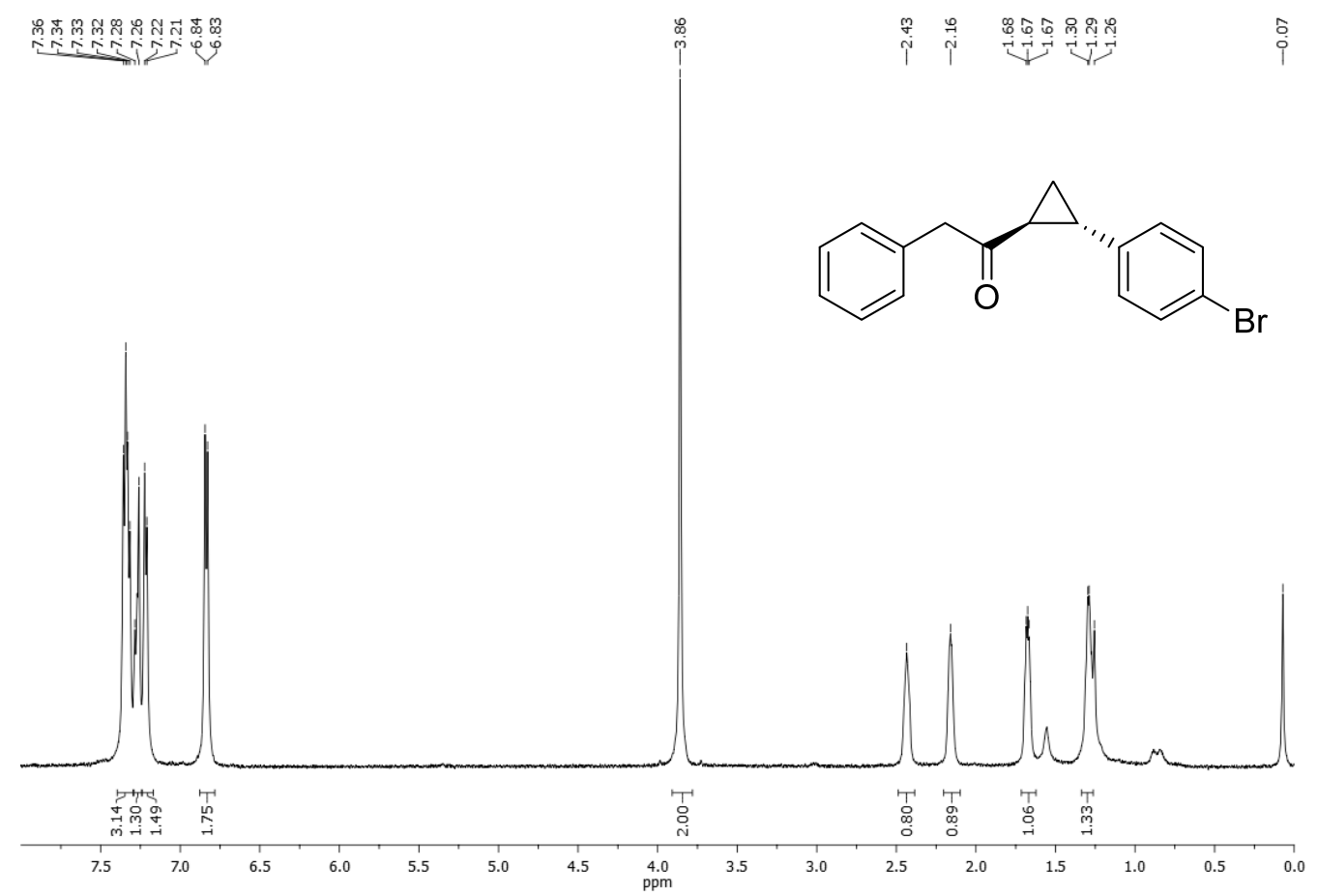

1-((1S, 2S)-2-(4-bromophenyl)cyclopropyl)-2-phenylethan-1-one (5d), ${ }^{13} \mathrm{C}$ NMR (126 MHz, $\left.\mathrm{CDCl}_{3}\right)$ :

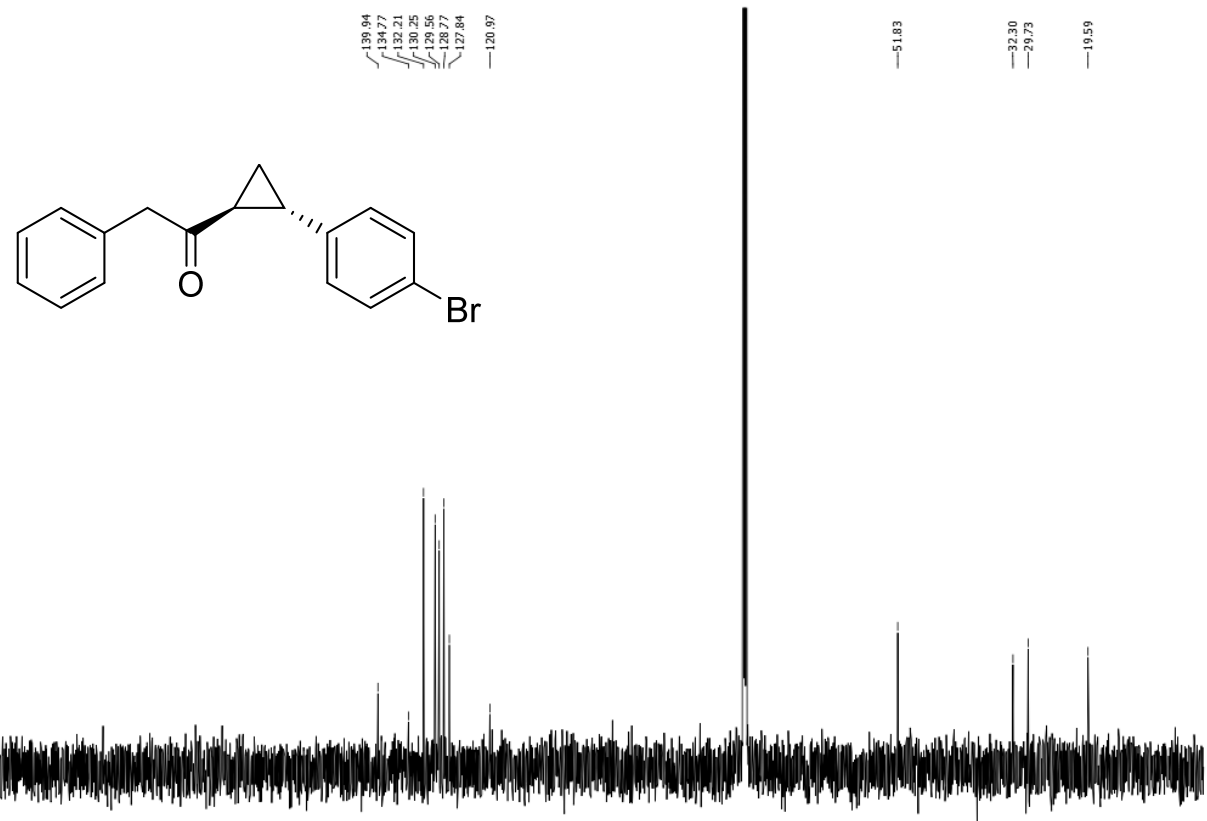

$\begin{array}{llllllllllllllllllllllllllllllll}220 & 210 & 200 & 190 & 180 & 170 & 160 & 150 & 140 & 130 & 120 & 110 & 100 & 90 & 80 & 70 & 60 & 50 & 40 & 30 & 20 & 10 & 0\end{array}$ 
1-((1S, 2S)-2-(4-(trifluoromethyl)phenyl)cyclopropyl)-2-phenylethan-1-one (5e), ${ }^{1} \mathrm{H}$ NMR $(500$ $\left.\mathrm{MHz}, \mathrm{CDCl}_{3}\right)$ :

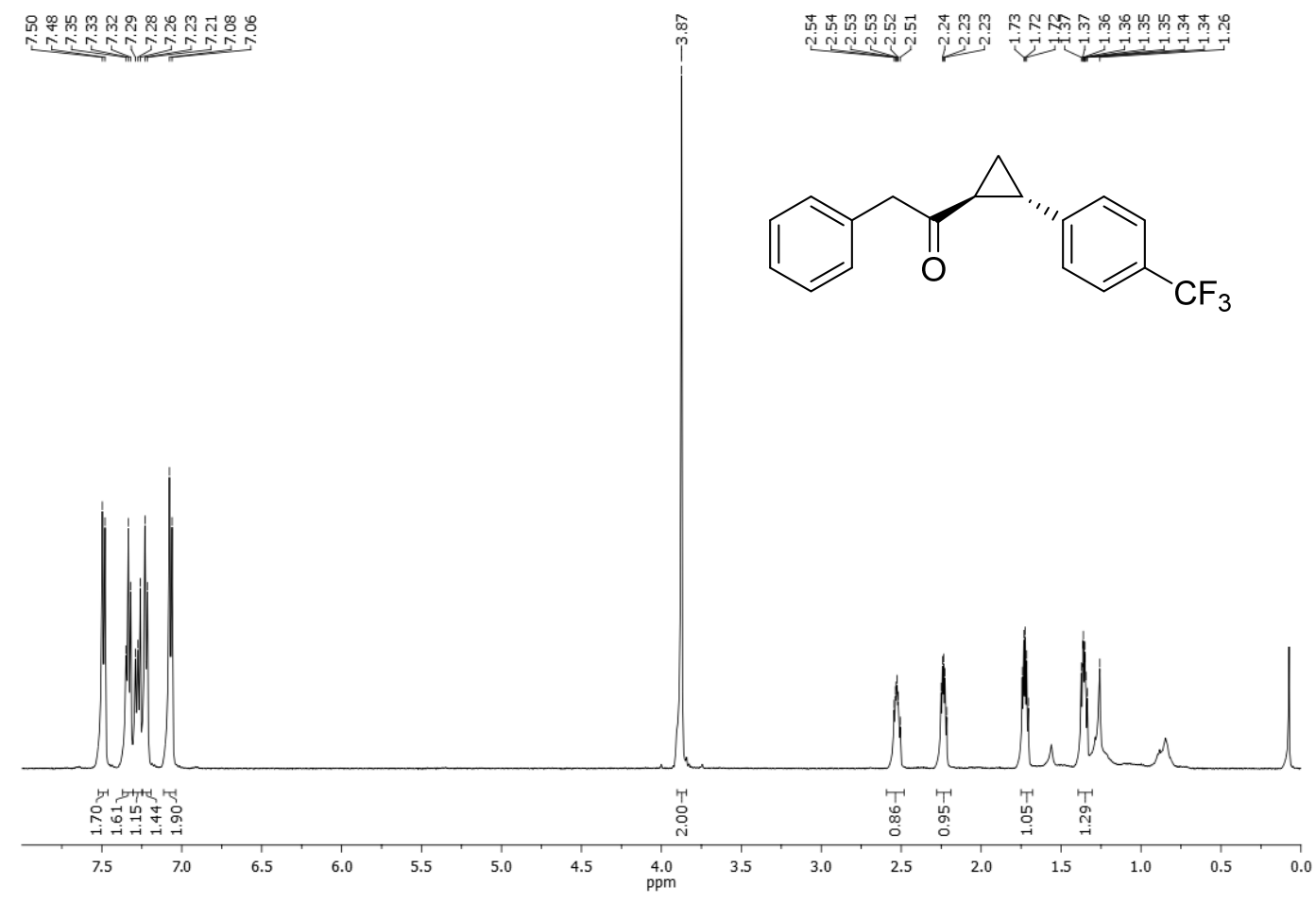

1-((1S, 2S)-2-(4-(trifluoromethyl)phenyl)cyclopropyl)-2-phenylethan-1-one (5e), ${ }^{13} \mathrm{C}$ NMR (126 $\left.\mathrm{MHz}, \mathrm{CDCl}_{3}\right)$ :

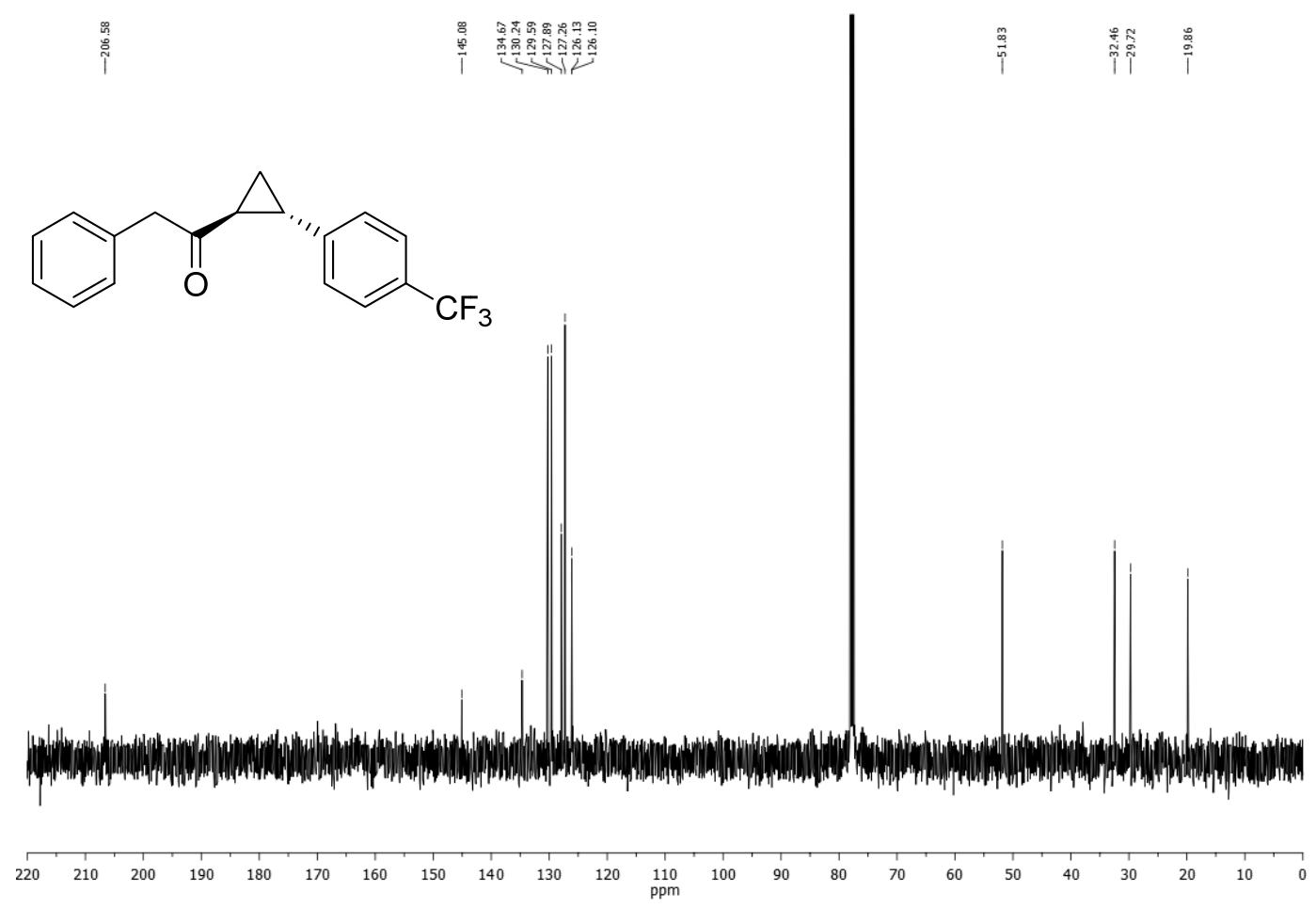


1-((1S, 2S)-2-(4-(Trifluoromethyl)phenyl)cyclopropyl)-2-phenylethan-1-one (5e), ${ }^{19} \mathrm{~F}$ NMR (376 $\left.\mathrm{MHz}, \mathrm{CDCl}_{3}\right)$ :

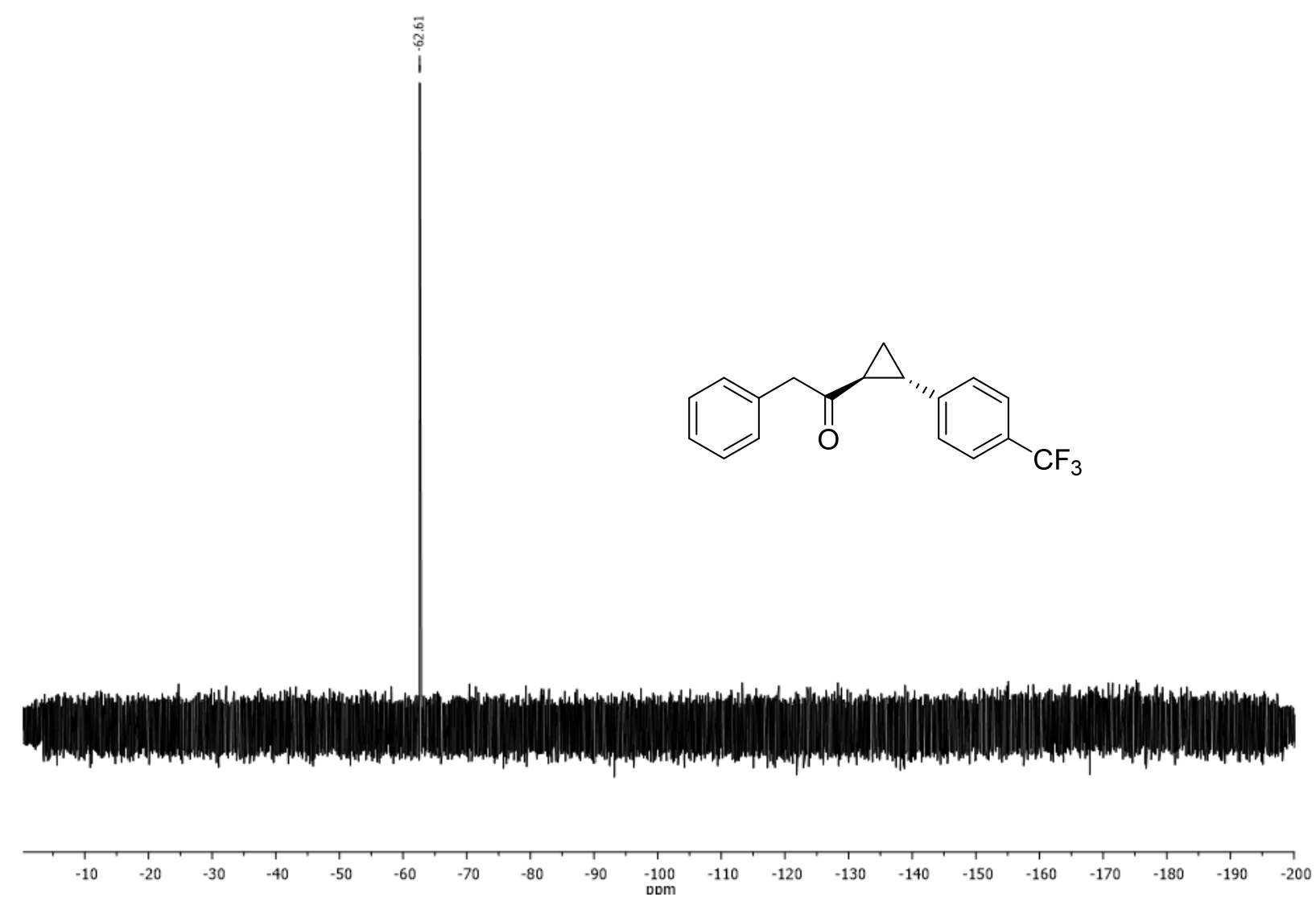


2-phenyl-1-((1S, 2S)-2-(m-tolyl)cyclopropyl)ethan-1-one (5f), ${ }^{1} \mathrm{H} \mathrm{NMR}\left(500 \mathrm{MHz}, \mathrm{CDCl}_{3}\right)$ :

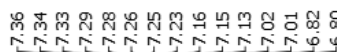

急

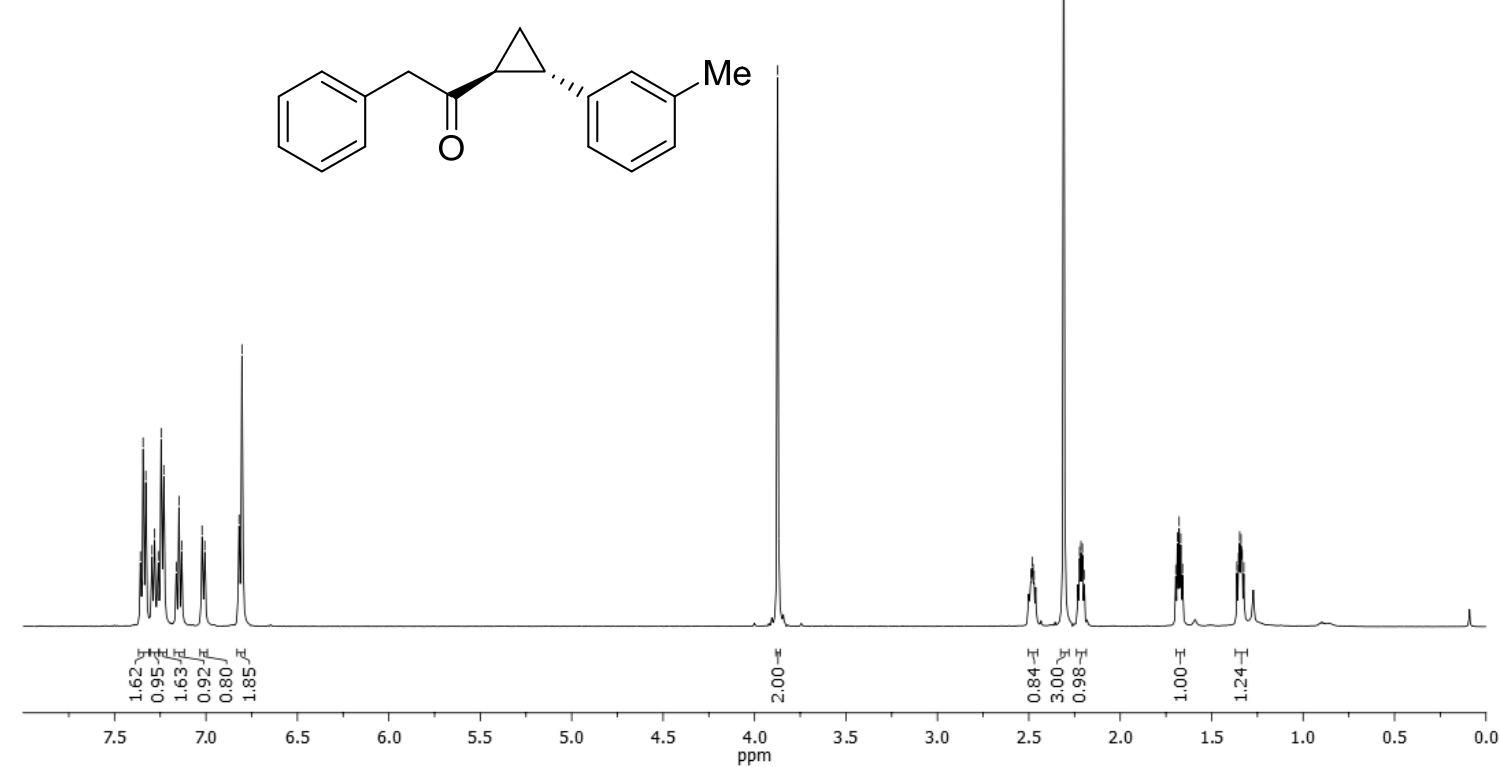

2-phenyl-1-((1S, 2S)-2-(m-tolyl)cyclopropyl)ethan-1-one (5f), ${ }^{13} \mathrm{C}$ NMR (126 MHz, $\left.\mathrm{CDCl}_{3}\right)$ :

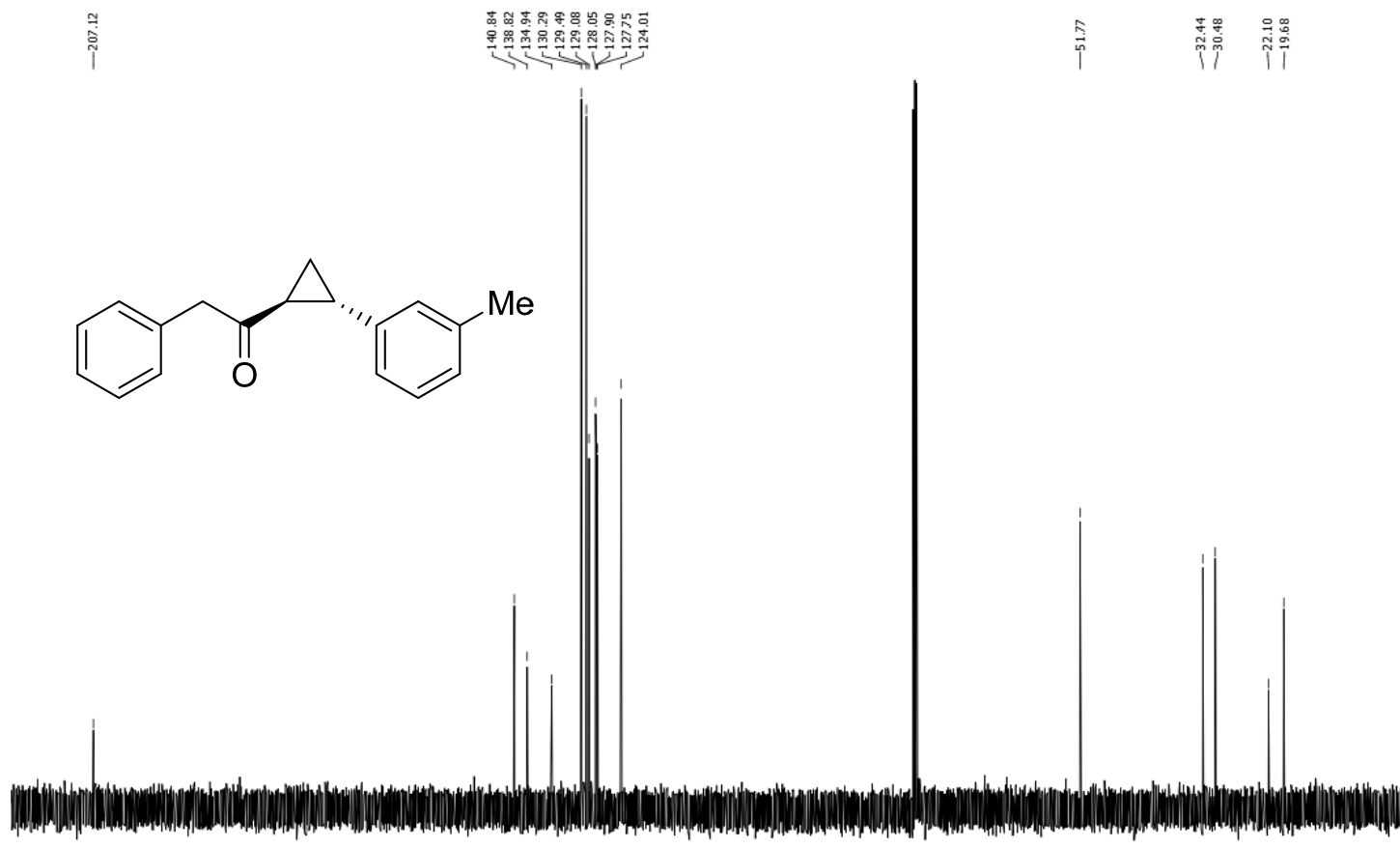

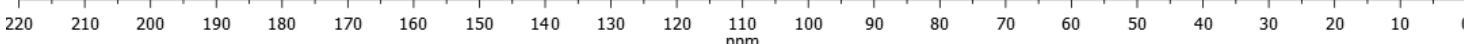


2-phenyl-1-((1S, 2S)-2-(o-tolyl)cyclopropyl)ethan-1-one (5g), ${ }^{1} \mathrm{H}$ NMR (500 MHz, $\left.\mathrm{CDCl}_{3}\right)$ :

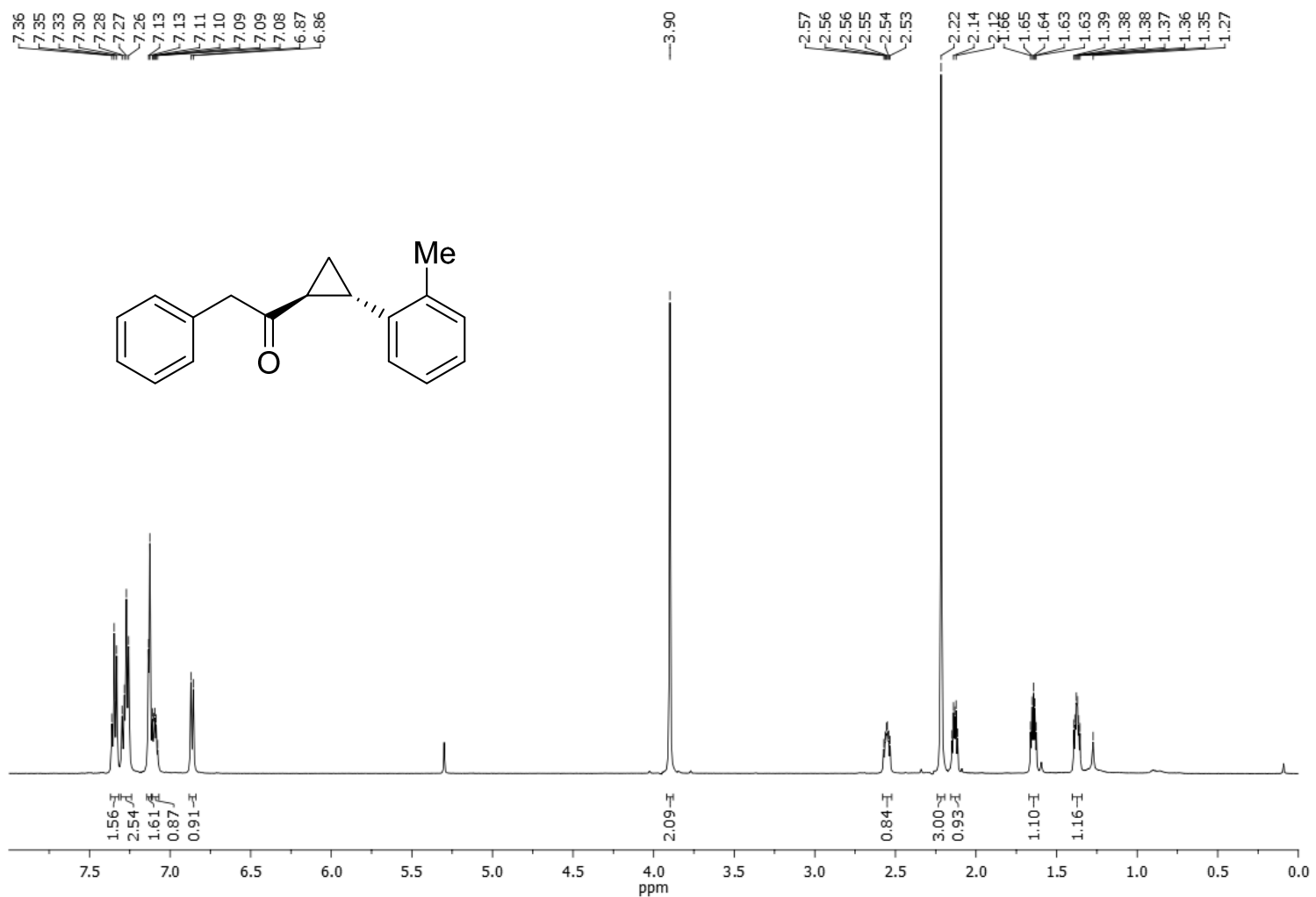

2-phenyl-1-((1S, 2S)-2-(o-tolyl)cyclopropyl)ethan-1-one (5g), ${ }^{13} \mathrm{C} \mathrm{NMR}\left(126 \mathrm{MHz}, \mathrm{CDCl}_{3}\right)$ :

กิ

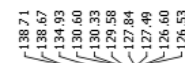

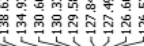

至<smiles>Cc1ccccc1[C@@H]1C[C@H]1C(=O)Cc1ccccc1</smiles>
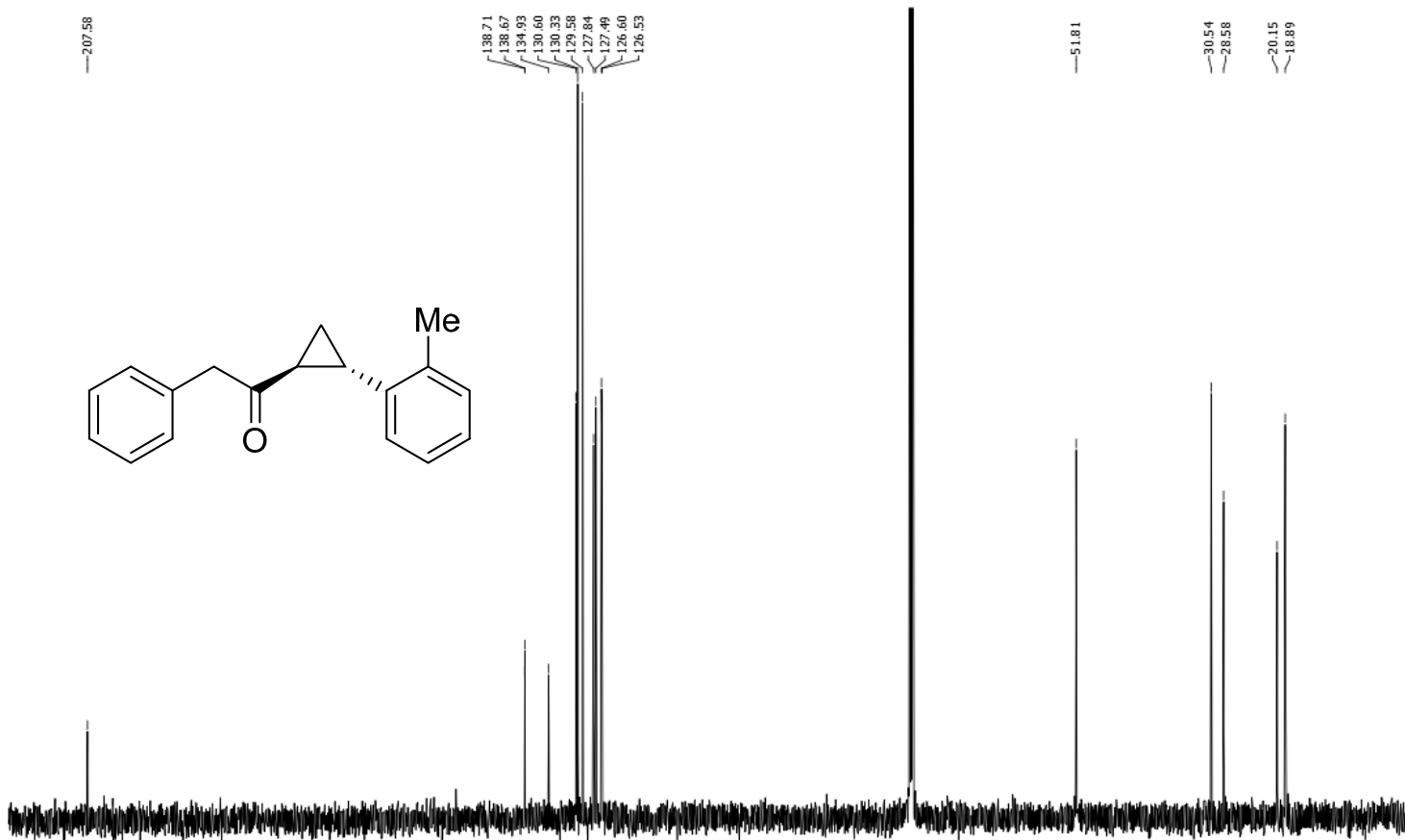

\begin{tabular}{lllllllllllllllllllllllllll}
\hline 220 & 210 & 200 & 190 & 180 & 170 & 160 & 150 & 140 & 130 & 120 & 110 & 100 & 90 & 80 & 70 & 60 & 50 & 40 & 30 & 20 & 10 & 0
\end{tabular} 
1-((1S, 2S)-2-methyl-2-phenylcyclopropyl)-2-phenylethan-1-one (5h), ${ }^{1} \mathrm{H}$ NMR (500 $\mathrm{MHz}$, $\left.\mathrm{CDCl}_{3}\right)$ :
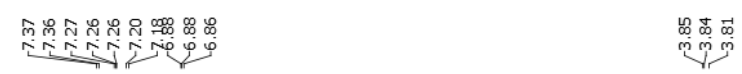

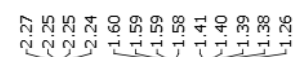

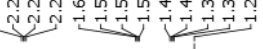<smiles>CC1(c2ccccc2)CC1C(=O)Cc1ccccc1</smiles>

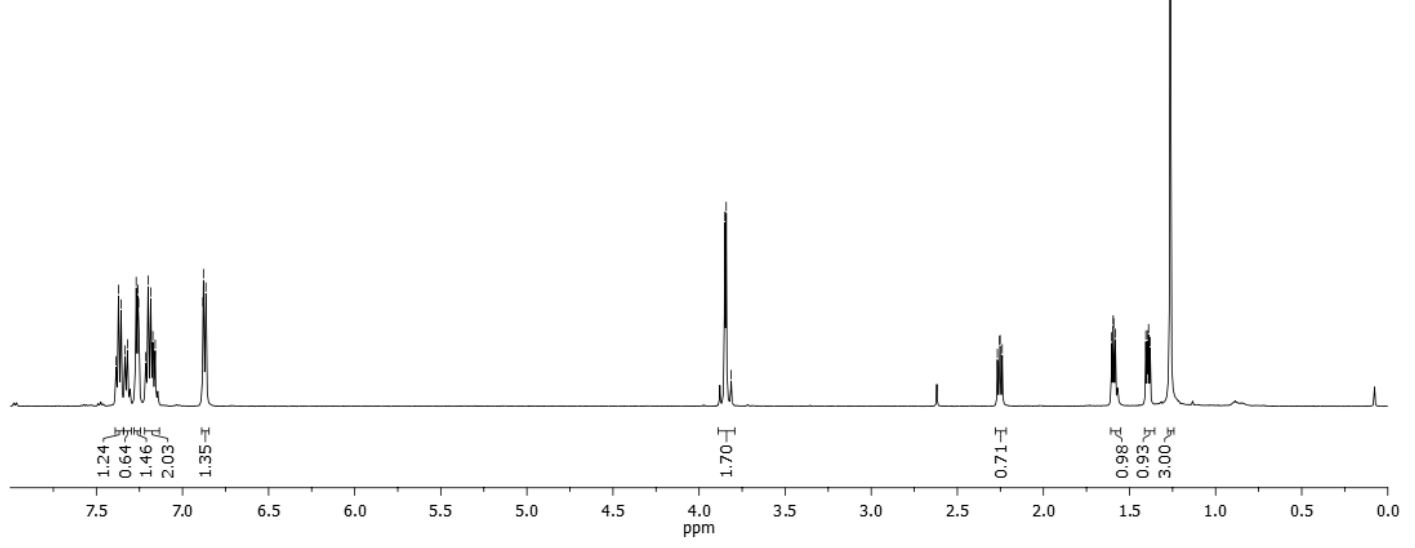

1-((1S, 2S)-2-methyl-2-phenylcyclopropyl)-2-phenylethan-1-one (5h), ${ }^{13} \mathrm{C}$ NMR (126 MHz, $\left.\mathrm{CDCl}_{3}\right)$ :

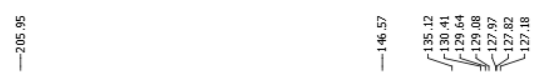<smiles>C[C@]1(c2ccccc2)C[C@H]1C(=O)Cc1ccccc1</smiles>

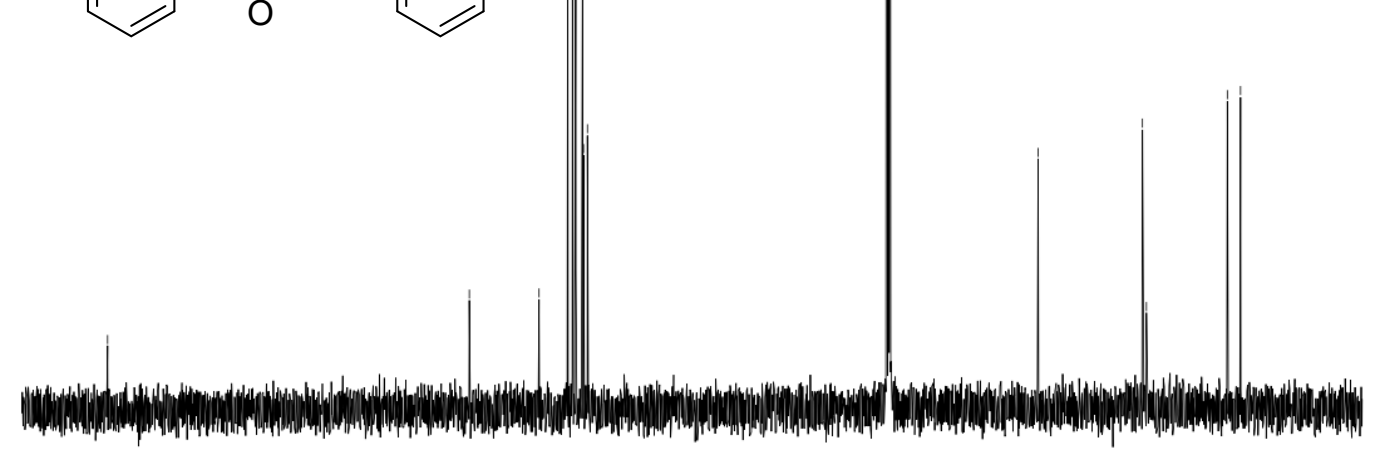

\begin{tabular}{llllllllllllllllllllllllllll}
\hline 220 & 210 & 200 & 190 & 180 & 170 & 160 & 150 & 140 & 130 & 120 & 110 & 100 & 90 & 80 & 70 & 60 & 50 & 40 & 30 & 20 & 10 & 0
\end{tabular} 
1-((1S, 2S)-2-(perfluorophenyl)cyclopropyl)-2-phenylethan-1-one (5i), ${ }^{1} \mathrm{H}$ NMR (500 MHz, $\left.\mathrm{CDCl}_{3}\right): \square$

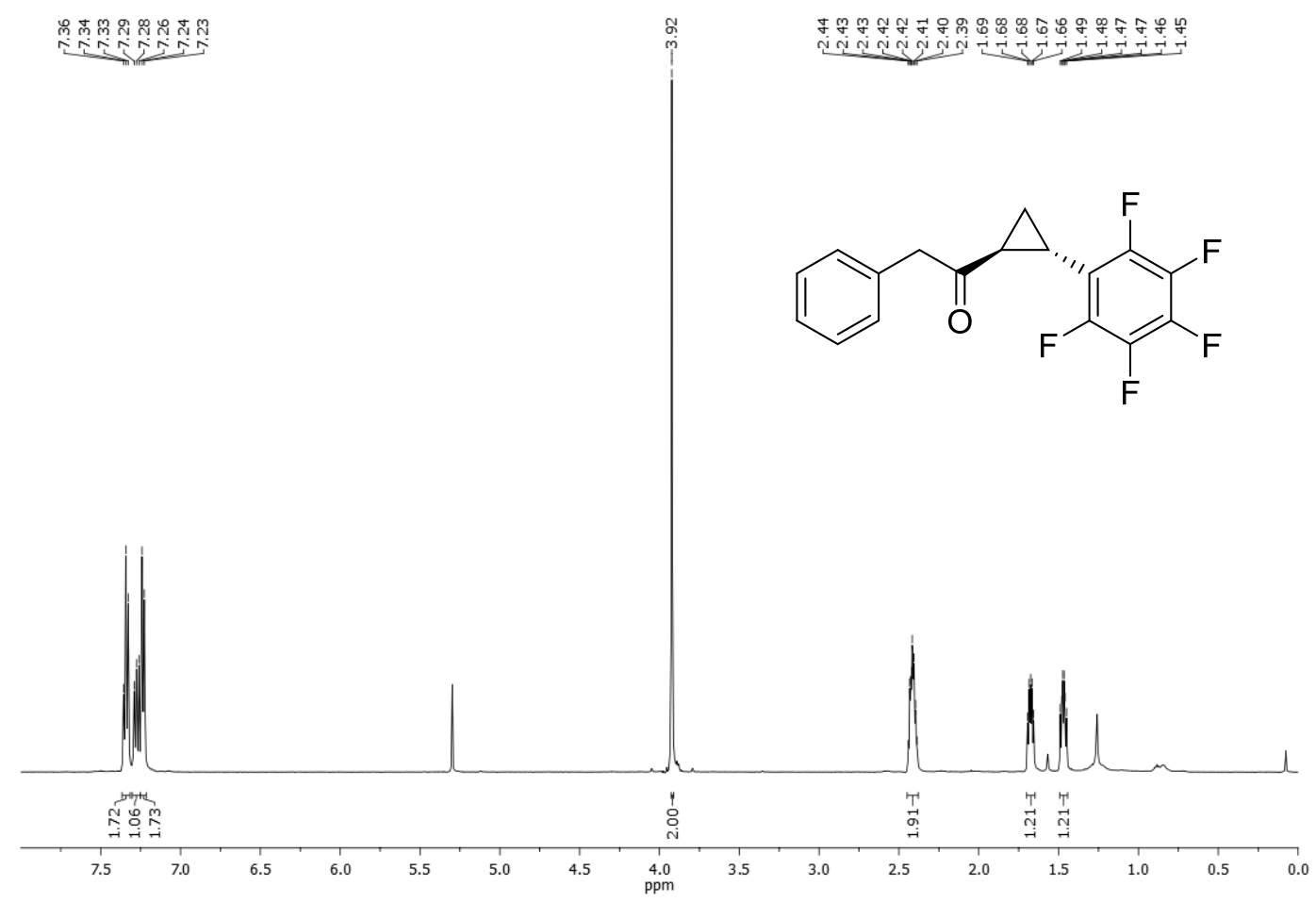

1-((1S, 2S)-2-(perfluorophenyl)cyclopropyl)-2-phenylethan-1-one (5i), ${ }^{13} \mathrm{C}$ NMR (126 MHz, $\left.\mathrm{CDCl}_{3}\right)$ :

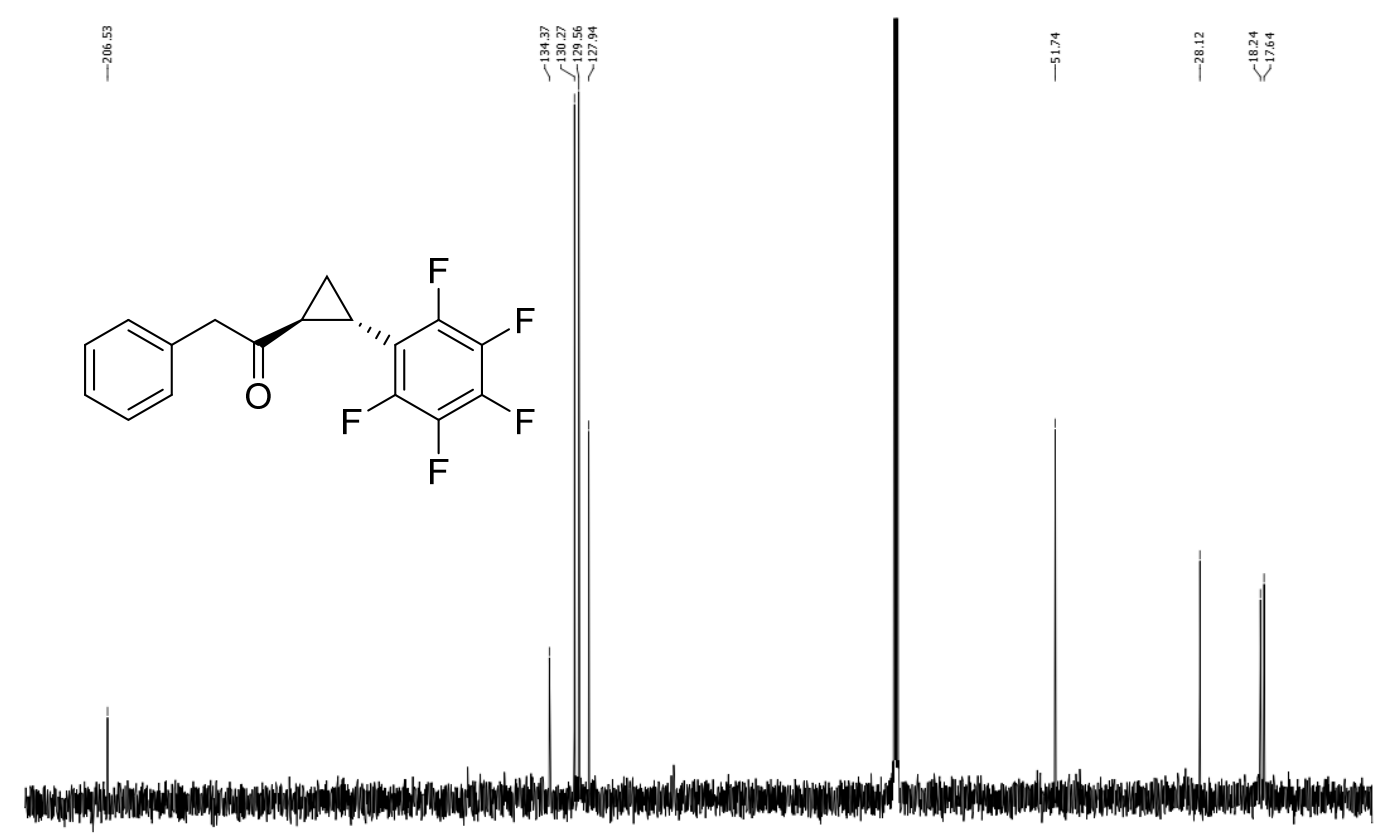

$\begin{array}{lllllllllllllllllllllllllll}220 & 210 & 200 & 190 & 180 & 170 & 160 & 150 & 140 & 130 & 120 & 110 & 100 & 90 & 80 & 70 & 60 & 50 & 40 & 30 & 20 & 10 & 0 \\ & & & \end{array}$ 
1-((1S, 2S)-2-(perfluorophenyl)cyclopropyl)-2-phenylethan-1-one (5i), ${ }^{19} \mathrm{~F}$ NMR (376 MHz, $\left.\mathrm{CDCl}_{3}\right)$ :

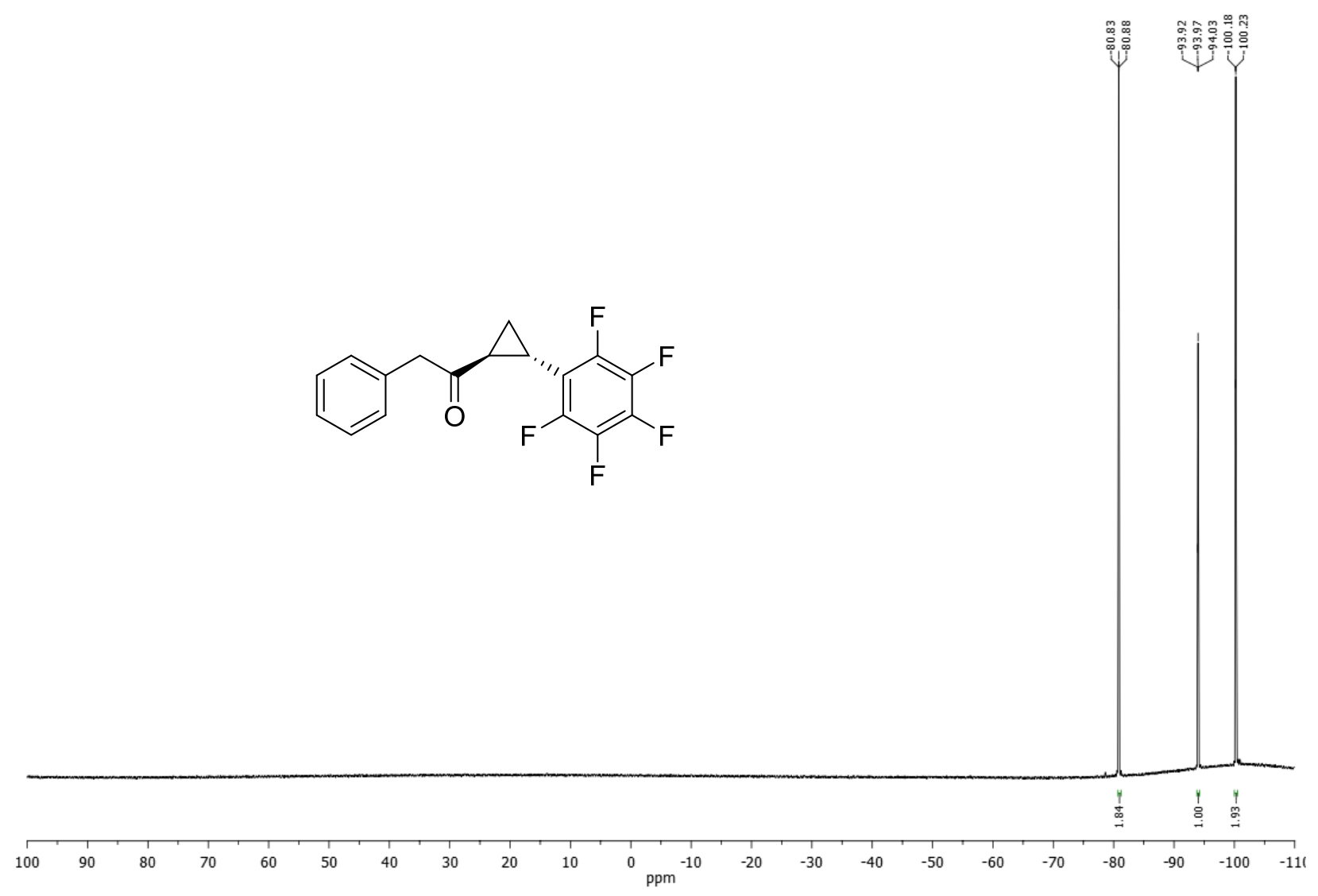


1-((1S, 2S)-2-methyl-2-(thiophen-3-yl)cyclopropyl)-2-phenylethan-1-one (5j), ${ }^{1} \mathrm{H}$ NMR (500 $\left.\mathrm{MHz}, \mathrm{CDCl}_{3}\right): \square$
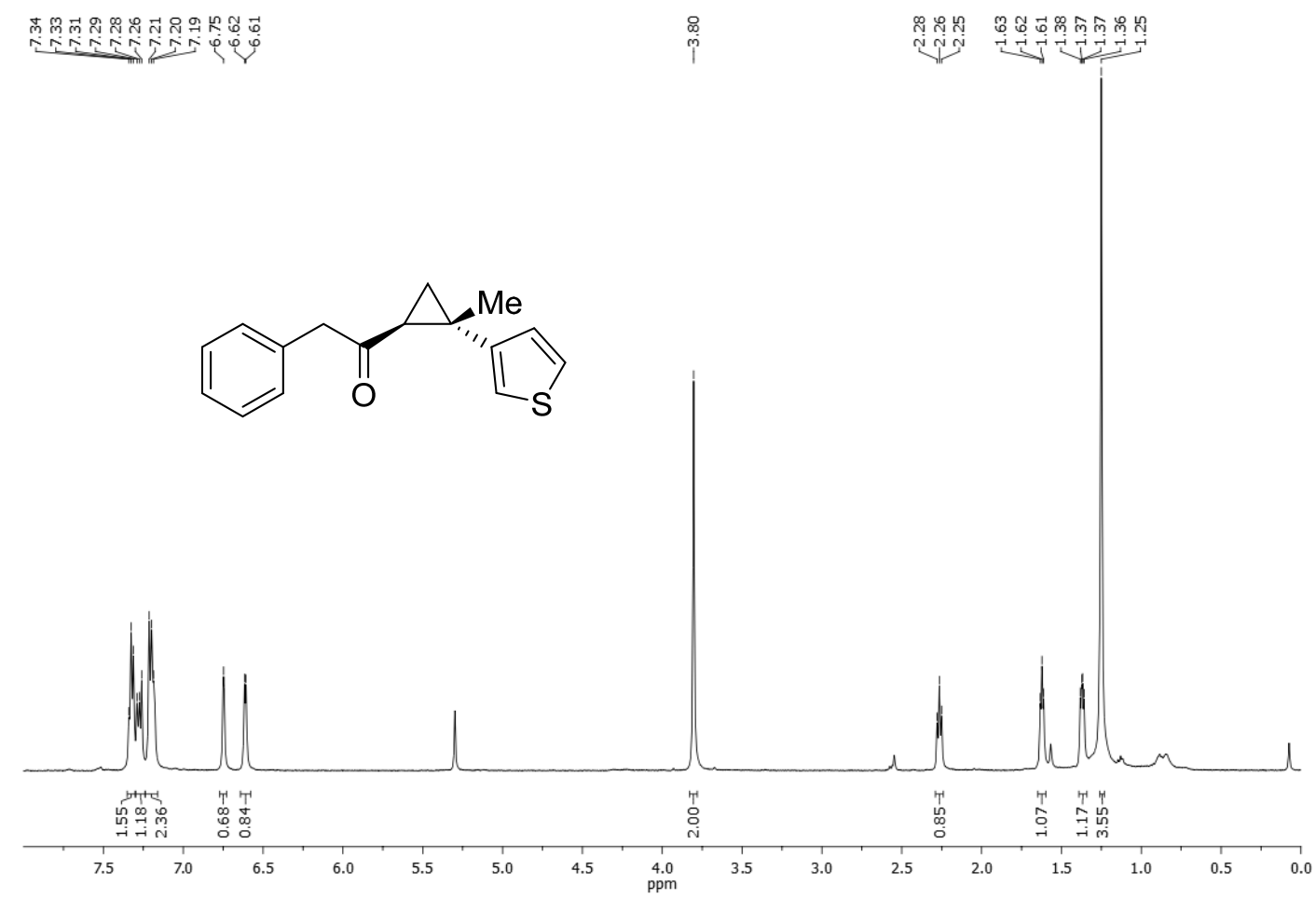

1-((1S, 2S)-2-methyl-2-(thiophen-3-yl)cyclopropyl)-2-phenylethan-1-one (5j), ${ }^{13} \mathrm{C}$ NMR (126 $\left.\mathrm{MHz}, \mathrm{CDCl}_{3}\right)$ :<smiles>CC1(c2ccsc2)CC1C(=O)Cc1ccccc1</smiles>

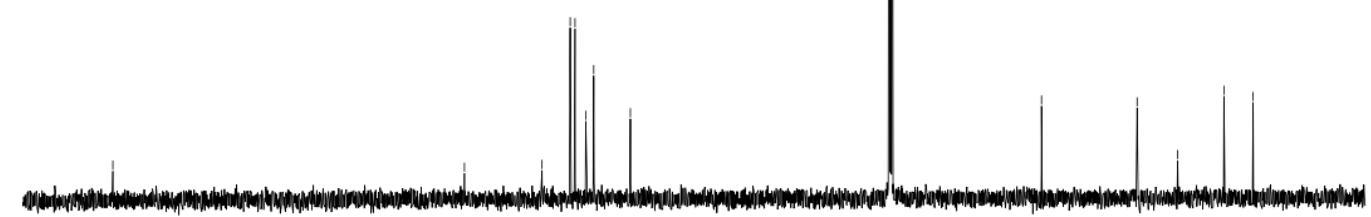

\begin{tabular}{lllllllllllllllllllllllllll}
\hline 220 & 210 & 200 & 190 & 180 & 170 & 160 & 150 & 140 & 130 & 120 & 110 & 100 & 90 & 80 & 70 & 60 & 50 & 40 & 30 & 20 & 10 & 0
\end{tabular} 
1-((1S, 2S)-2-methyl-2-(pyridine-2-yl)cyclopropyl)-2-phenylethan-1-one (5k), ${ }^{1} \mathrm{H}$ NMR (500 $\left.\mathrm{MHz}, \mathrm{CDCl}_{3}\right)$ :

要

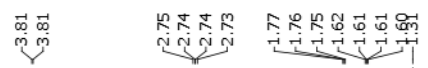<smiles>C[C@]1(c2ccccn2)C[C@H]1C(=O)Cc1ccccc1</smiles>

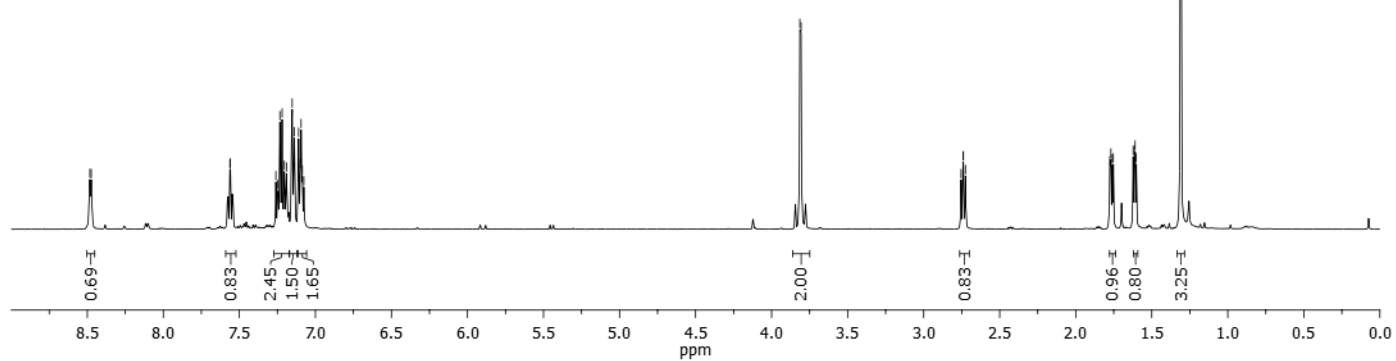

1-((1S, 2S)-2-methyl-2-(pyridine-2-yl)cyclopropyl)-2-phenylethan-1-one (5k), ${ }^{13} \mathrm{C}$ NMR (126 $\left.\mathrm{MHz}, \mathrm{CDCl}_{3}\right)$ :

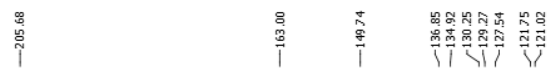<smiles>C[C@]1(c2ccccn2)CC1C(=O)Cc1ccccc1</smiles>

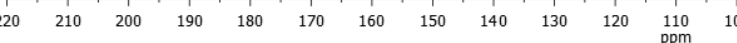


2-(4-chlorophenyl)-1-((1S, 2S)-2-phenylcyclopropyl)ethan-1-one (7a), ${ }^{1} \mathrm{H}$ NMR (500 MHz, $\left.\mathrm{CDCl}_{3}\right)$ :

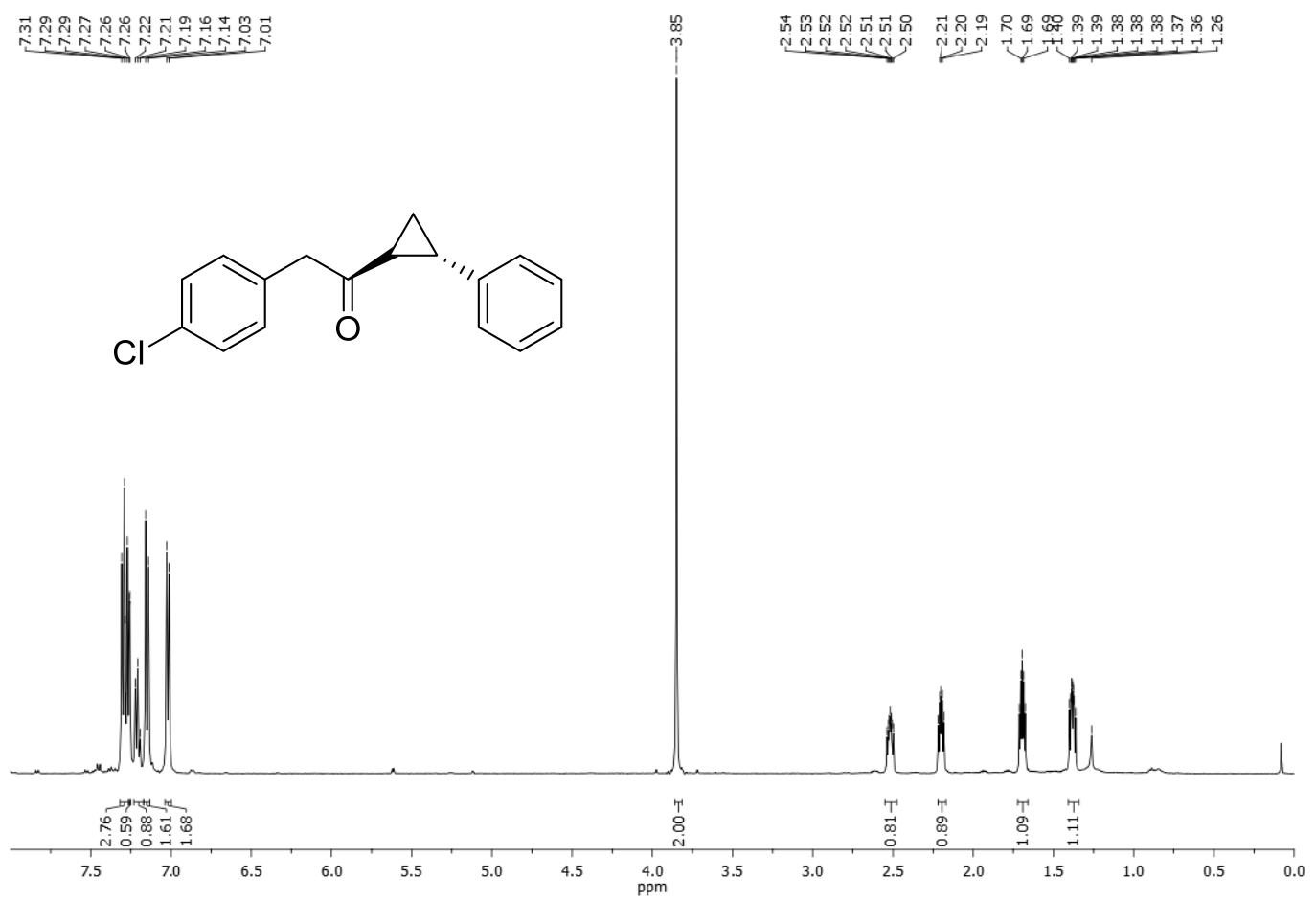

2-(4-chlorophenyl)-1-((1S, 2S)-2-phenylcyclopropyl)ethan-1-one (7a), ${ }^{13} \mathrm{C} \mathrm{NMR}(126 \mathrm{MHz}$, $\left.\mathrm{CDCl}_{3}\right)$ :

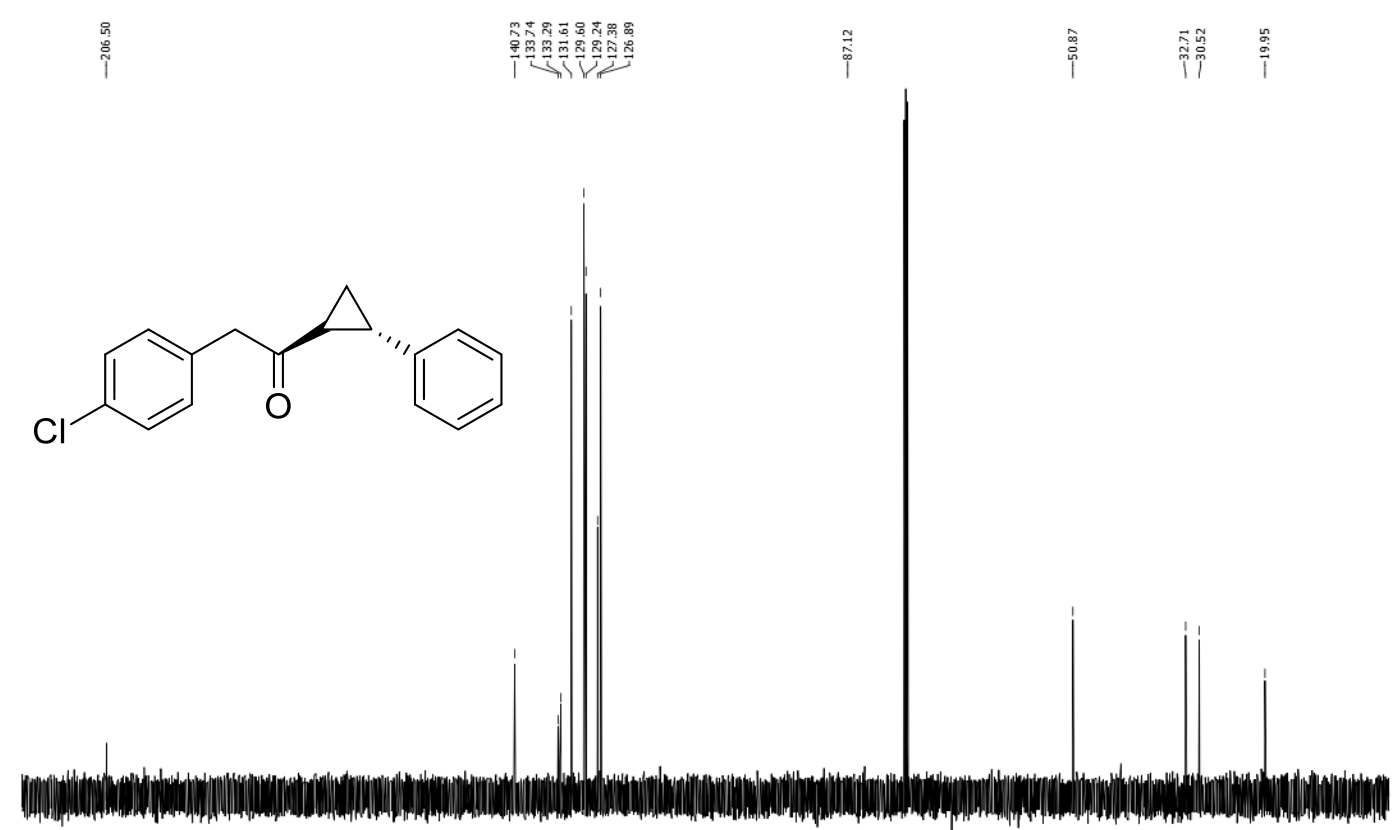

\begin{tabular}{llllllllllllllllllllllllll}
\hline 220 & 210 & 200 & 190 & 180 & 170 & 160 & 150 & 140 & 130 & 120 & 110 & 100 & 90 & 80 & 70 & 60 & 50 & 40 & 30 & 20 & 10 & 0 \\
& &
\end{tabular} 
2-(4-bromophenyl)-1-((1S, 2S)-2-phenylcyclopropyl)ethan-1-one (7b), ${ }^{1} \mathrm{H}$ NMR (500 MHz, $\left.\mathrm{CDCl}_{3}\right)$ :

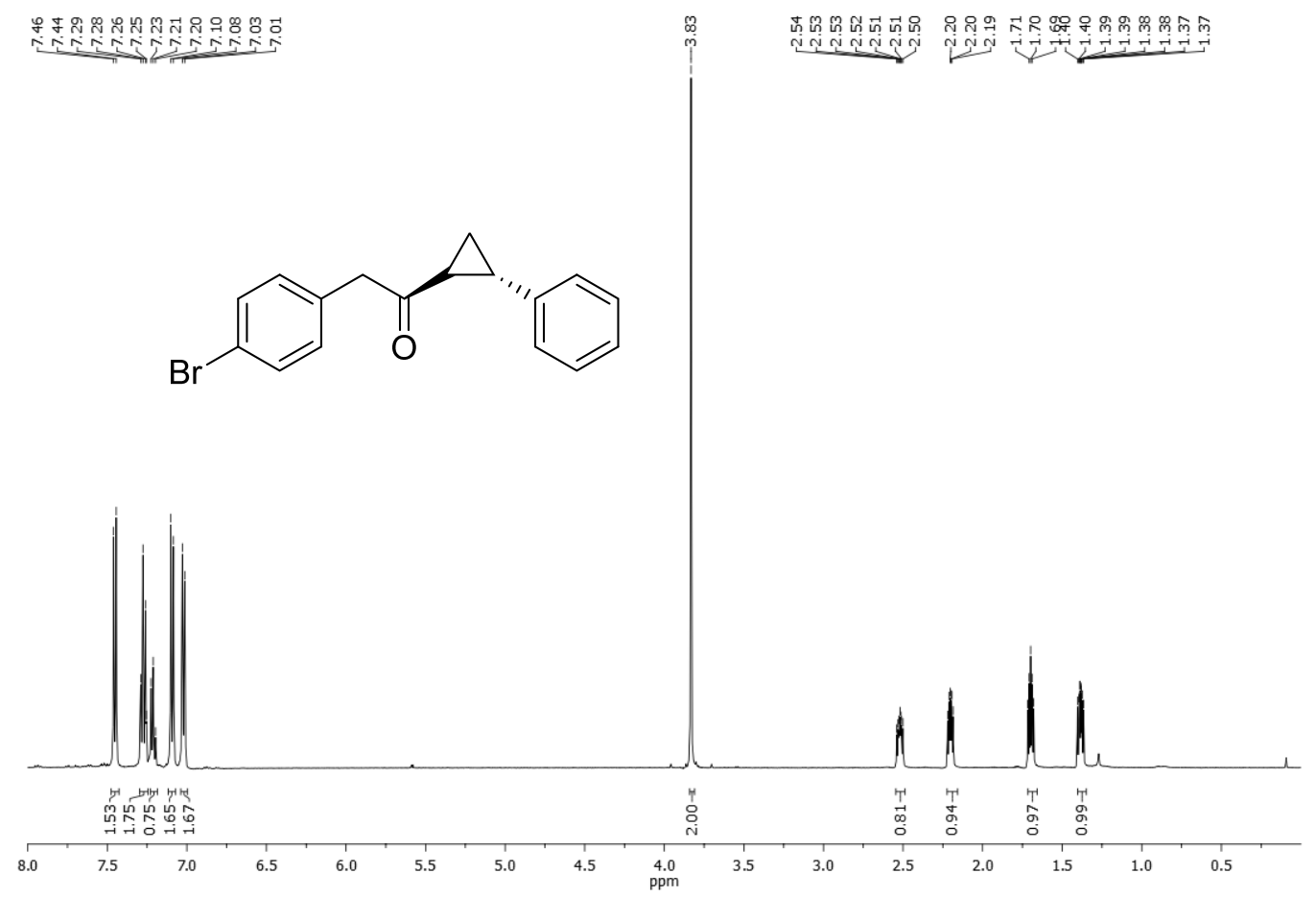

2-(4-bromophenyl)-1-((1S, 2S)-2-phenylcyclopropyl)ethan-1-one (7b), ${ }^{13} \mathrm{C}$ NMR (126 MHz, $\left.\mathrm{CDCl}_{3}\right)$ :

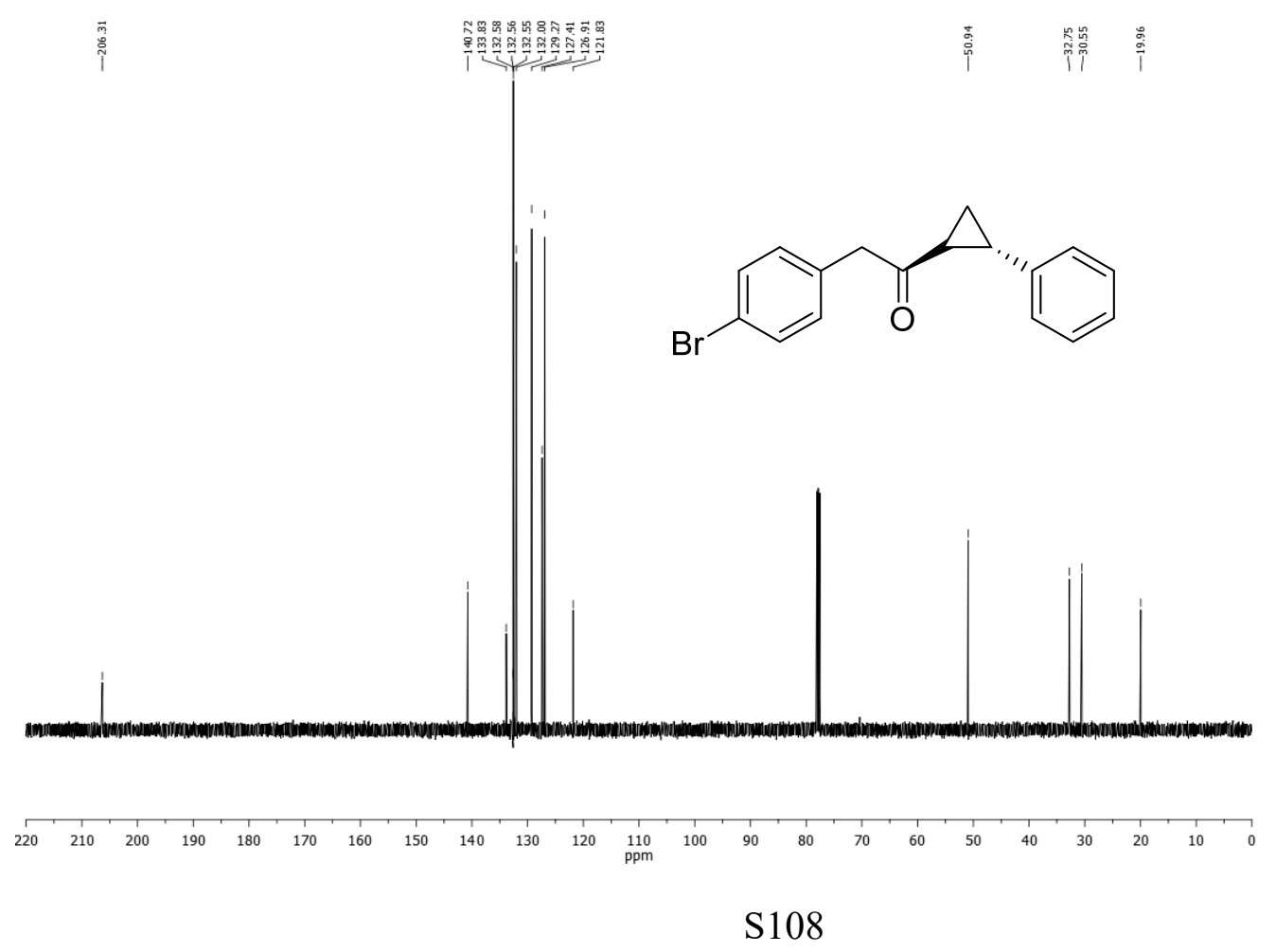


2-(3-bromophenyl)-1-((1S, 2S)-2-phenylcyclopropyl)ethan-1-one (7c), ${ }^{1} \mathrm{H}$ NMR (500 MHz, $\left.\mathrm{CDCl}_{3}\right)$ :

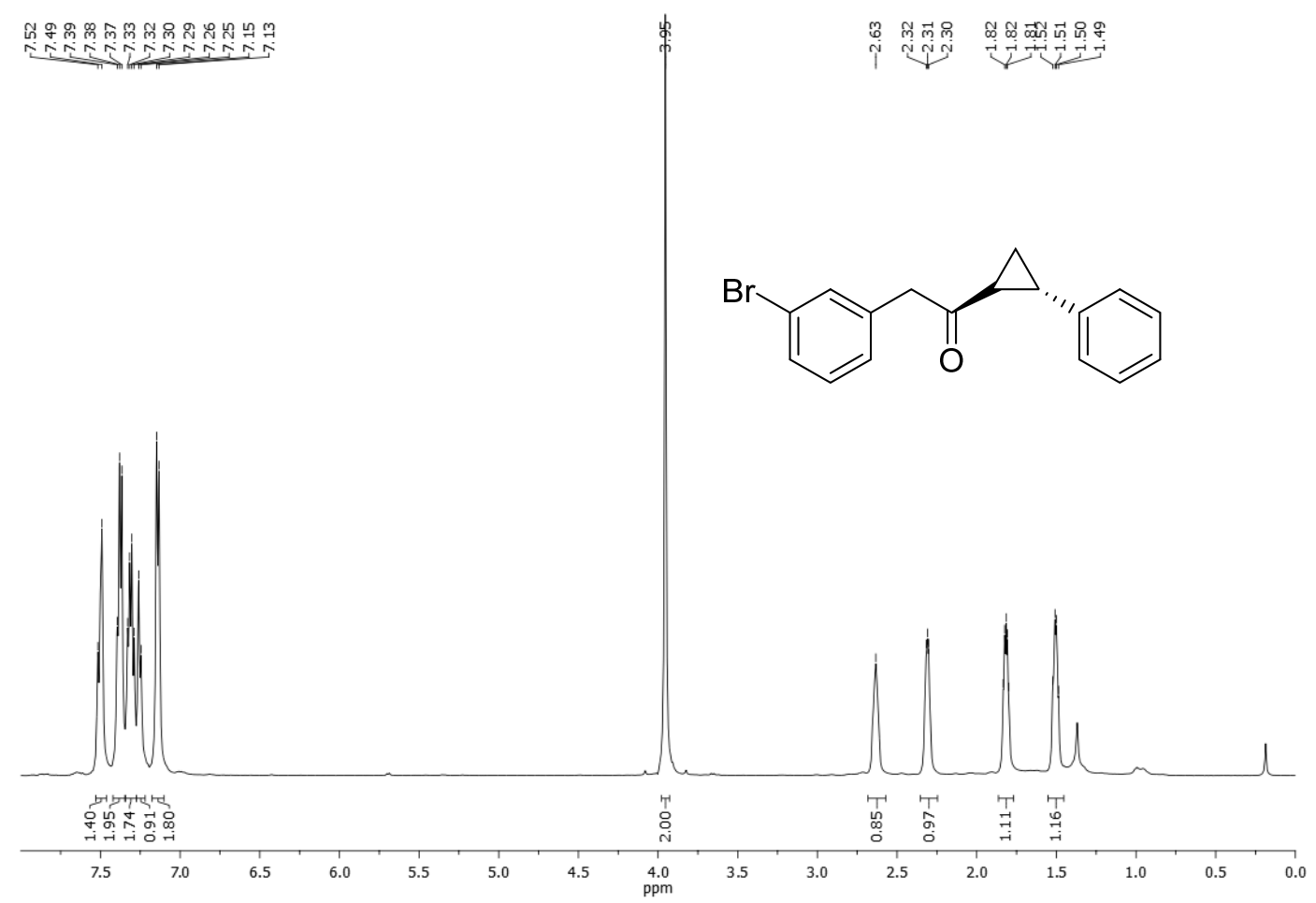

2-(3-bromophenyl)-1-((1S, 2S)-2-phenylcyclopropyl)ethan-1-one (7c), ${ }^{13} \mathrm{C}$ NMR (126 MHz, $\left.\mathrm{CDCl}_{3}\right)$ :

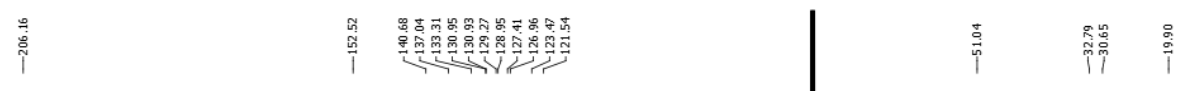<smiles>O=C(Cc1cccc(Br)c1)C1C[C@H]1c1ccccc1</smiles>

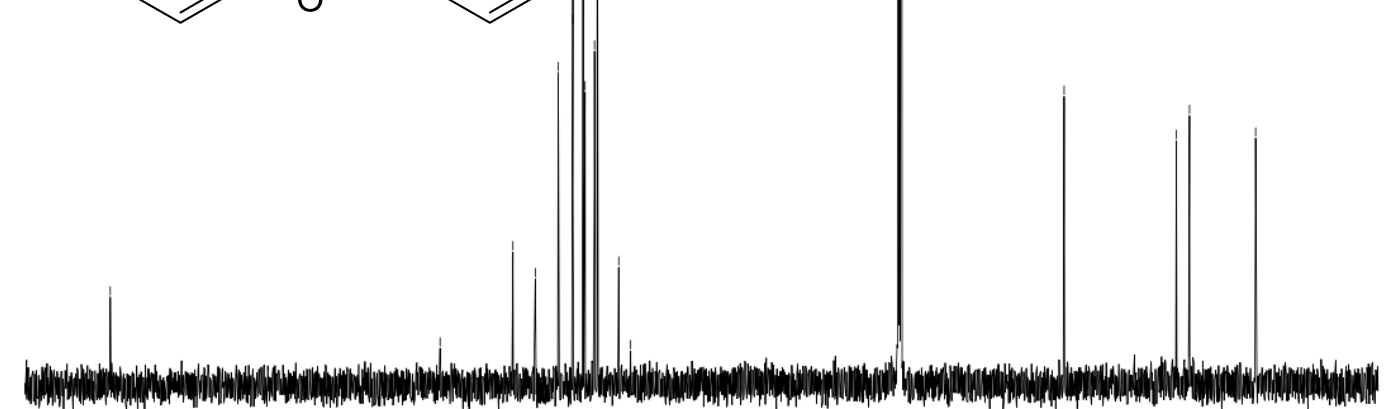

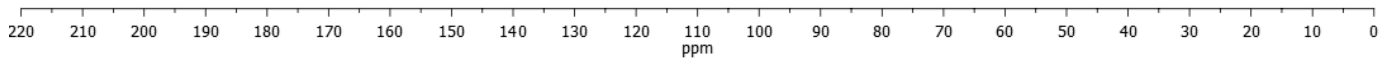


2-(2-bromophenyl)-1-((1S, 2S)-2-phenylcyclopropyl)ethan-1-one (7d), ${ }^{1} \mathrm{H}$ NMR (500 MHz, $\left.\mathrm{CDCl}_{3}\right)$ :

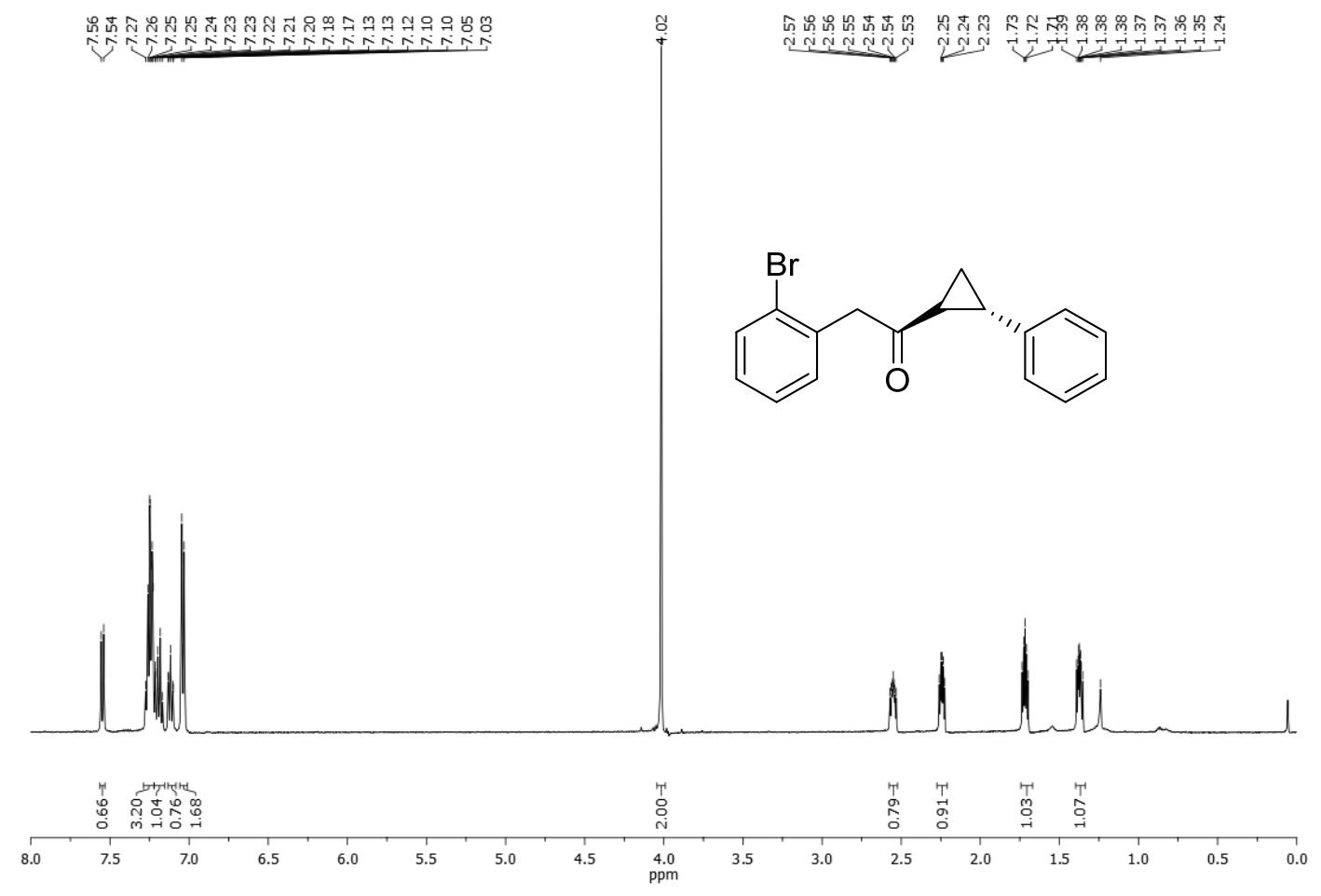

2-(2-bromophenyl)-1-((1S, 2S)-2-phenylcyclopropyl)ethan-1-one (7d), ${ }^{13} \mathrm{C}$ NMR (126 MHz, $\left.\mathrm{CDCl}_{3}\right)$ :
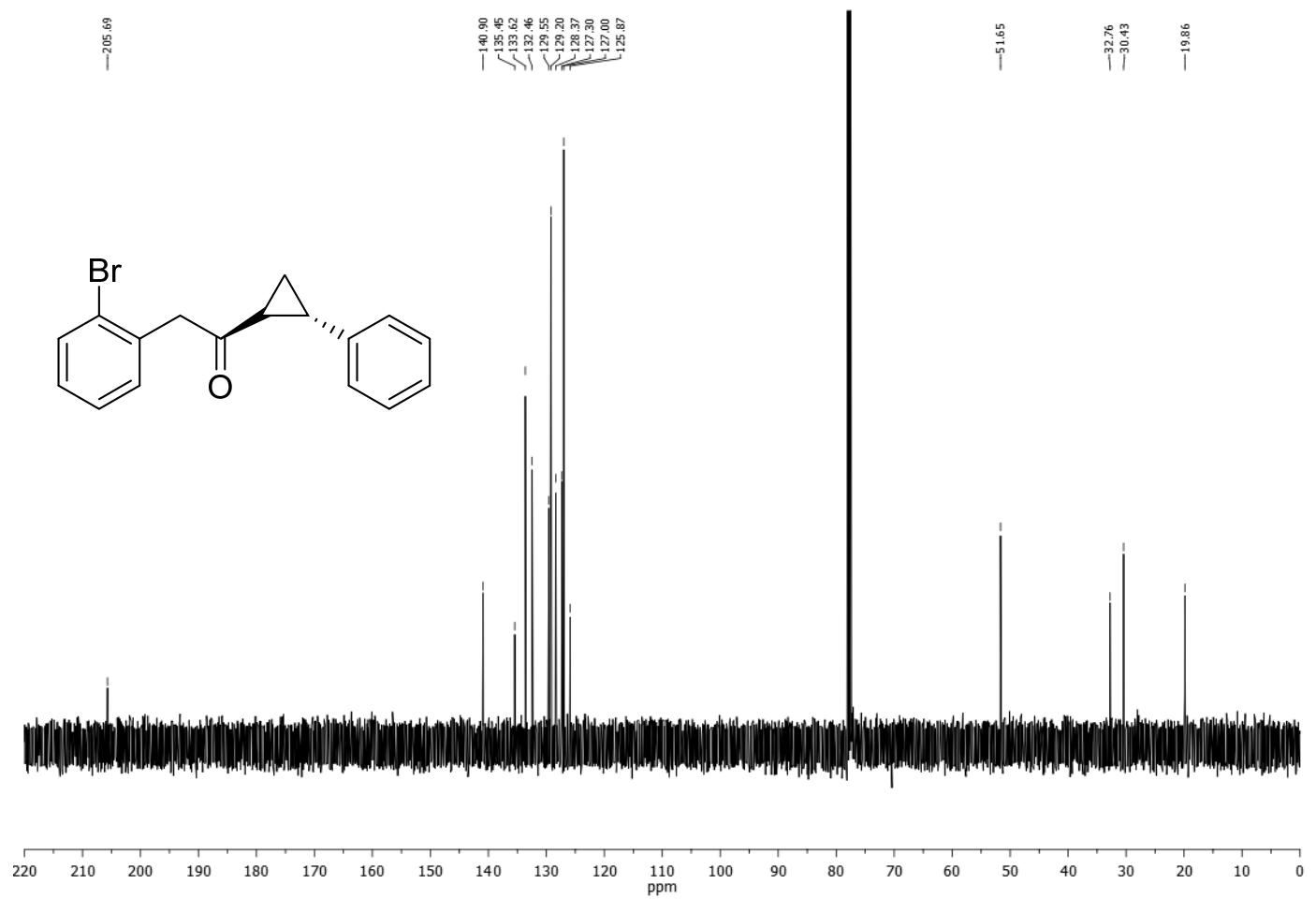
1-((1S, 2S)-2-phenylcyclopropyl)-2-(p-tolyl)ethan-1-one (7e), ${ }^{1} \mathrm{H}$ NMR (500 MHz, $\left.\mathrm{CDCl}_{3}\right)$ :

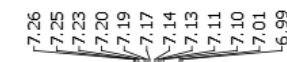

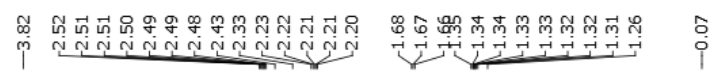

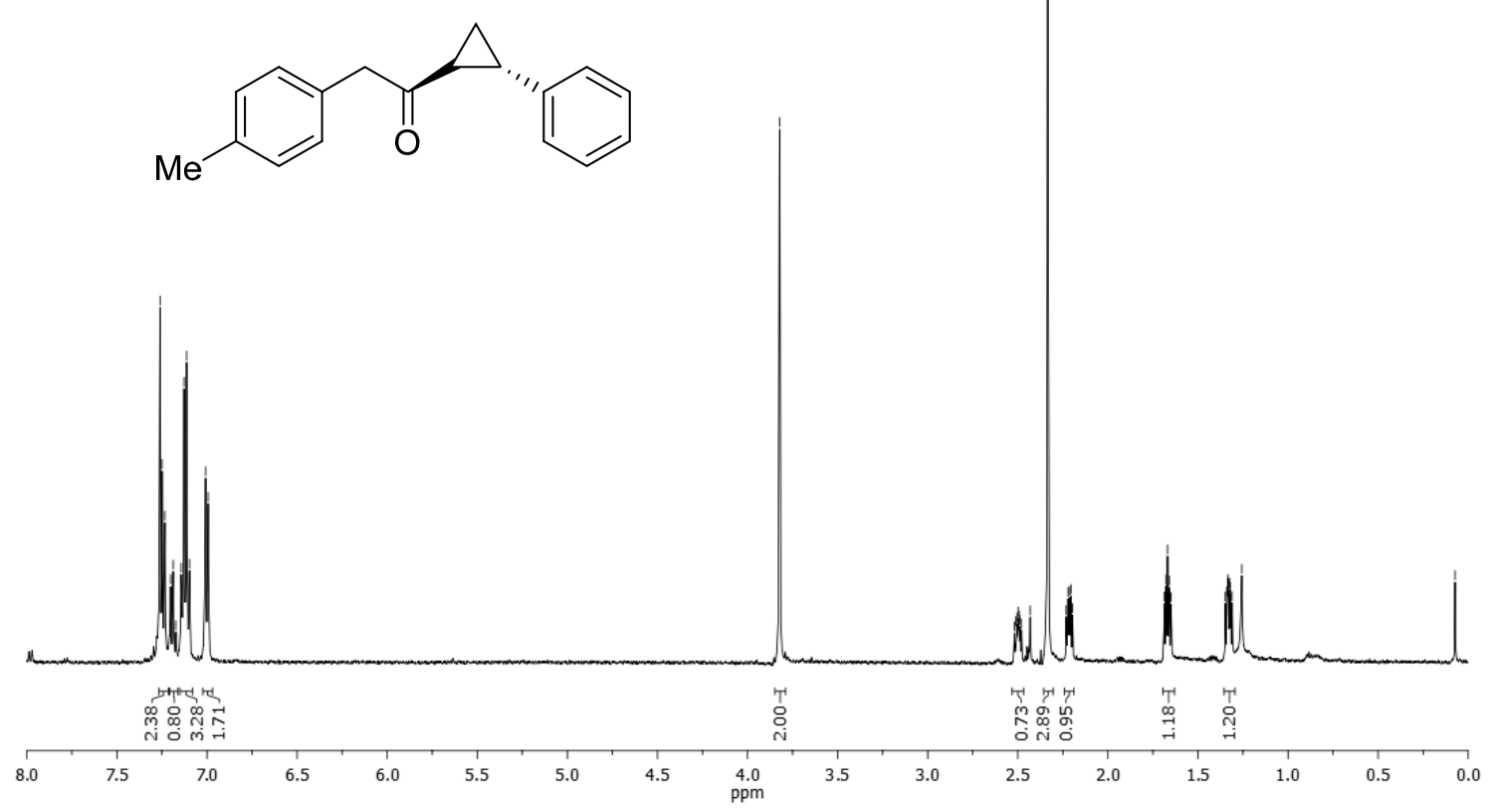

1-((1S, 2S)-2-phenylcyclopropyl)-2-(p-tolyl)ethan-1-one (7e), ${ }^{13} \mathrm{C}$ NMR $\left(126 \mathrm{MHz}, \mathrm{CDCl}_{3}\right)$ :

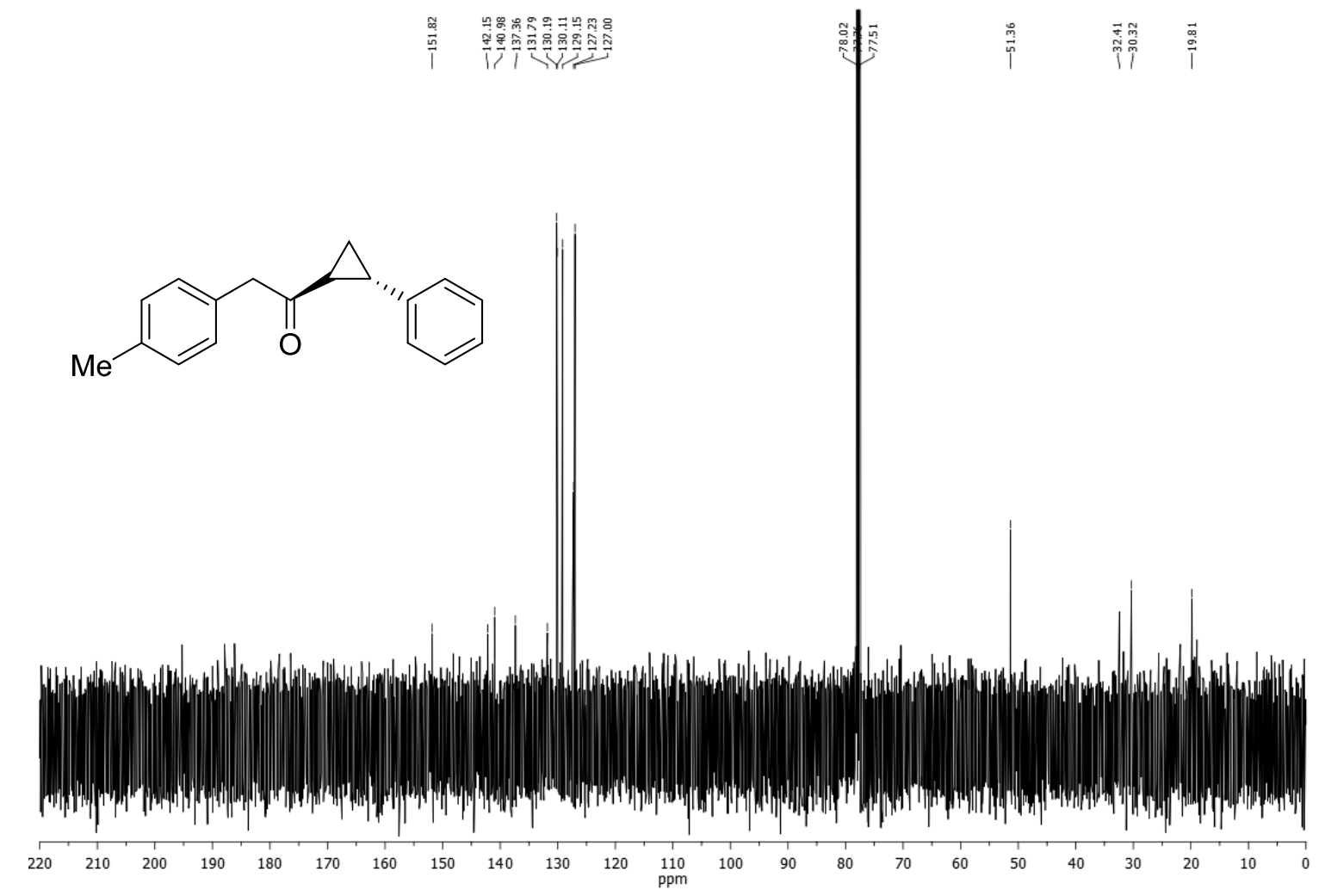


2-(4-methoxyphenyl)-1-((1S, 2S)-2-phenylcyclopropyl)ethan-1-one (7f), ${ }^{1} \mathrm{H}$ NMR (500 MHz, $\left.\mathrm{CDCl}_{3}\right)$ :

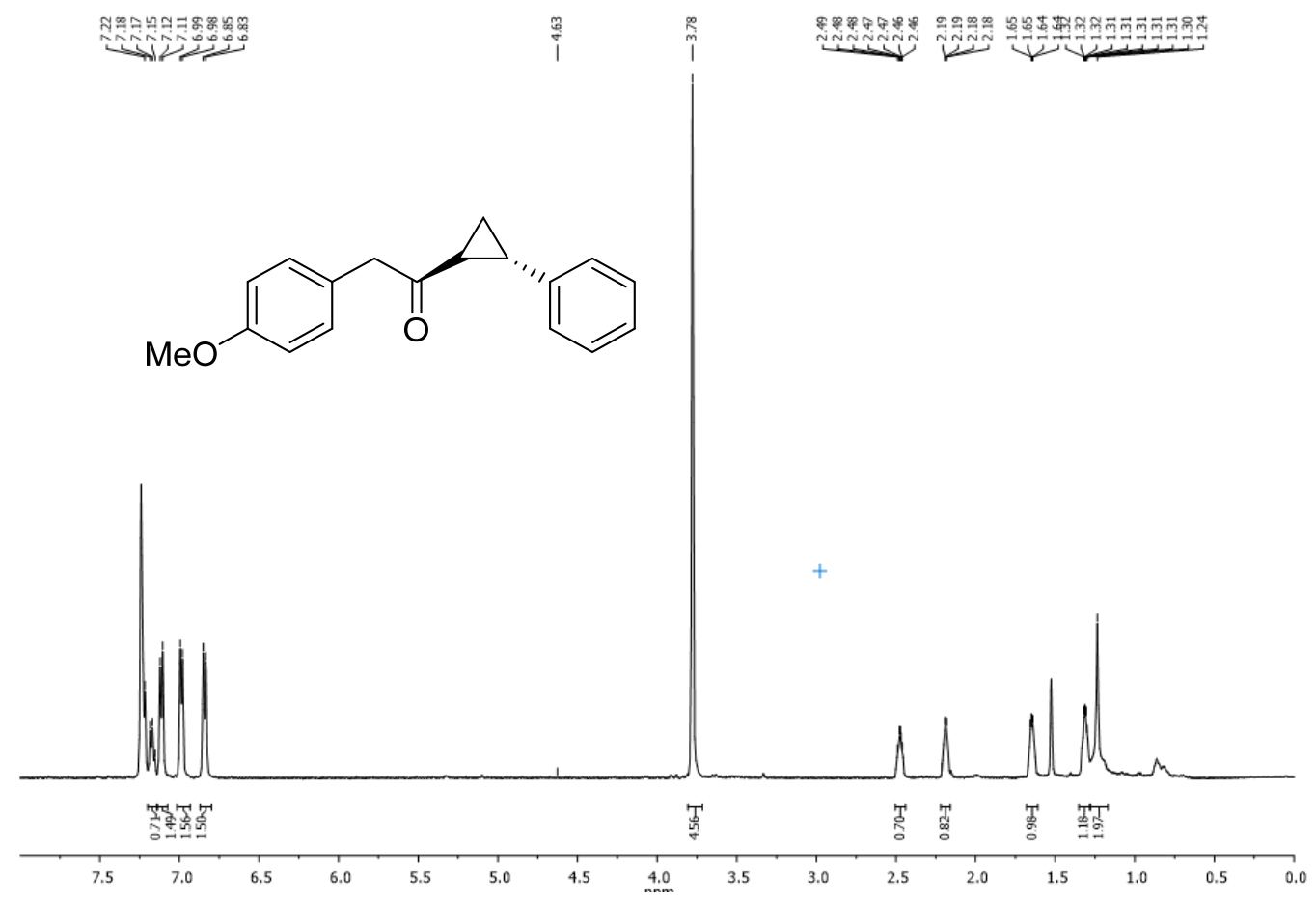

2-(4-methoxyphenyl)-1-((1S, 2S)-2-phenylcyclopropyl)ethan-1-one (7f), ${ }^{13} \mathrm{C}$ NMR (126 MHz, $\left.\mathrm{CDCl}_{3}\right)$ :
$\begin{array}{lll}5 & 8 \\ 5 & 0 & 0 \\ 1 & 1 & 0\end{array}$

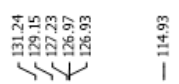
离

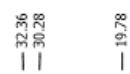<smiles>COc1ccc(CC(=O)C2C[C@H]2c2ccccc2)cc1</smiles>
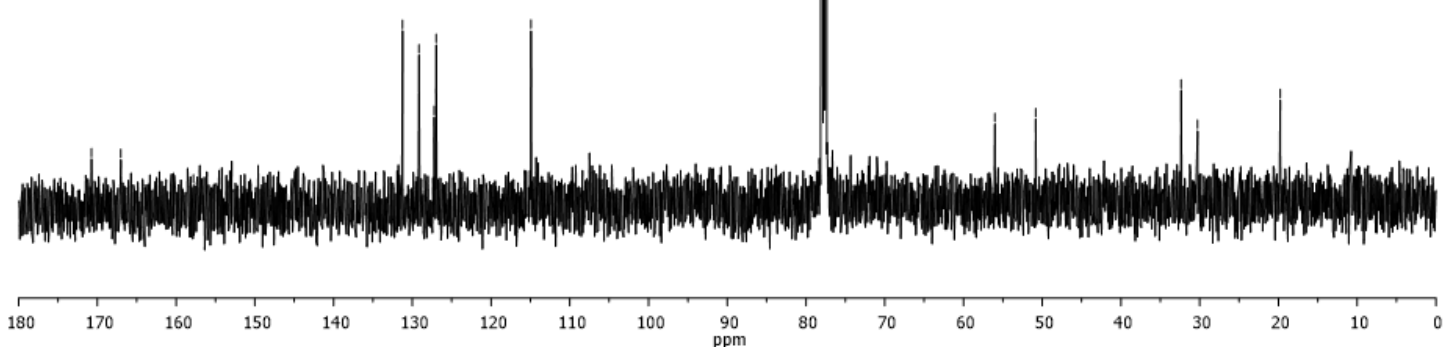
2-(3-methoxyphenyl)-1-((1S, 2S)-2-phenylcyclopropyl)ethan-1-one (7g), ${ }^{1} \mathrm{H}$ NMR (500 MHz, $\left.\mathrm{CDCl}_{3}\right)$ :

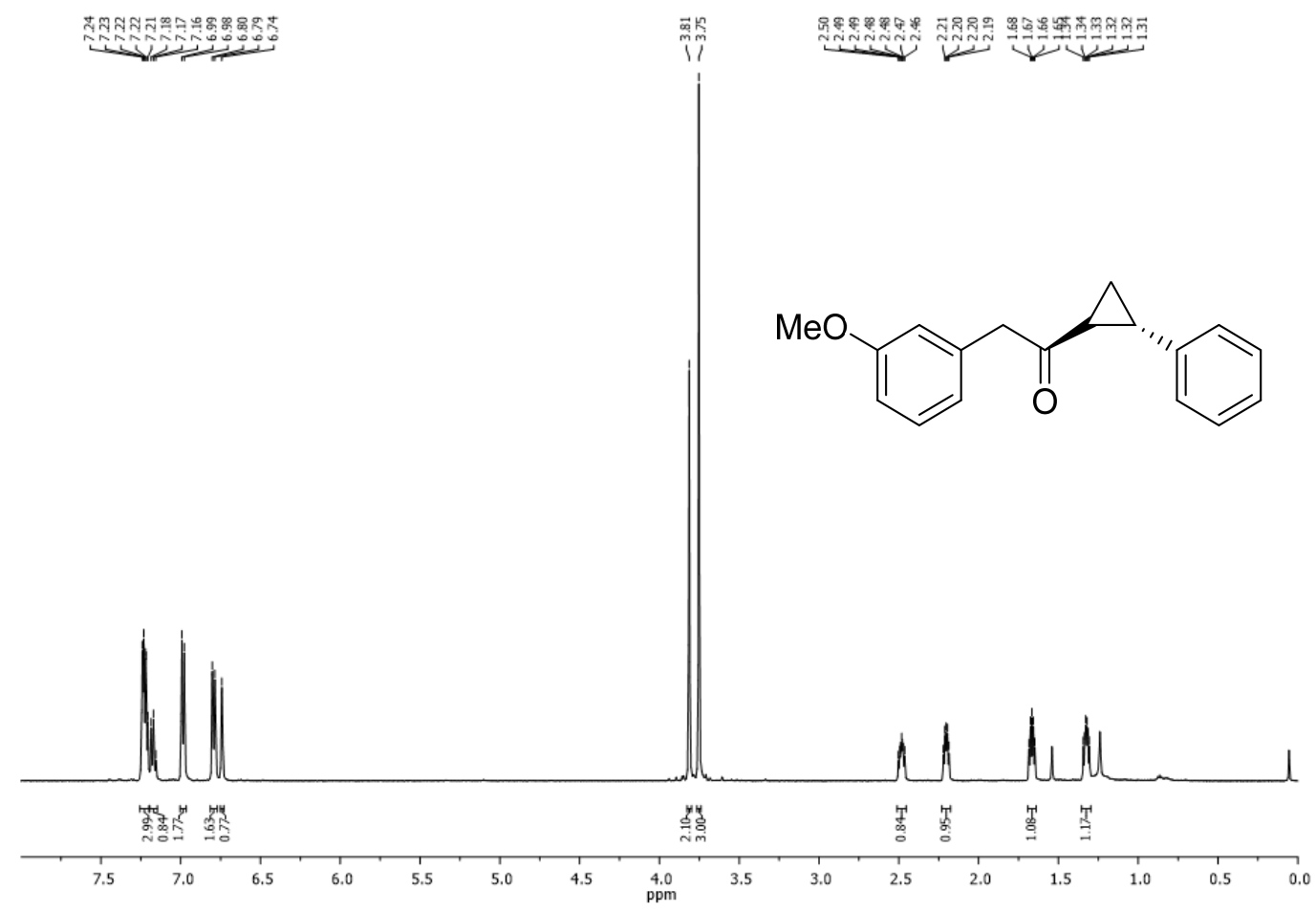

2-(3-methoxyphenyl)-1-((1S, 2S)-2-phenylcyclopropyl)ethan-1-one (7g), ${ }^{13} \mathrm{C}$ NMR (126 MHz, $\left.\mathrm{CDCl}_{3}\right)$ :<smiles>COc1cccc(CC(=O)[C@@H]2C[C@H]2c2ccccc2)c1</smiles>
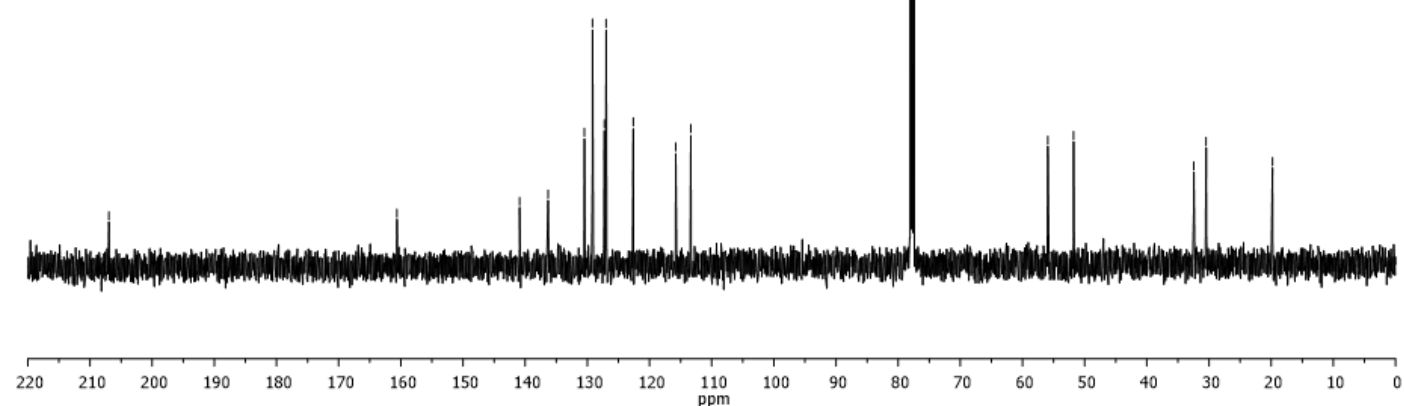
2-(benzo[1,3]dioxol-5-yl)-1-((1S, 2S)-2-phenylcyclopropyl)ethan-1-one (7h), ${ }^{1} \mathrm{H}$ NMR (500 MHz, $\left.\mathrm{CDCl}_{3}\right)$ :

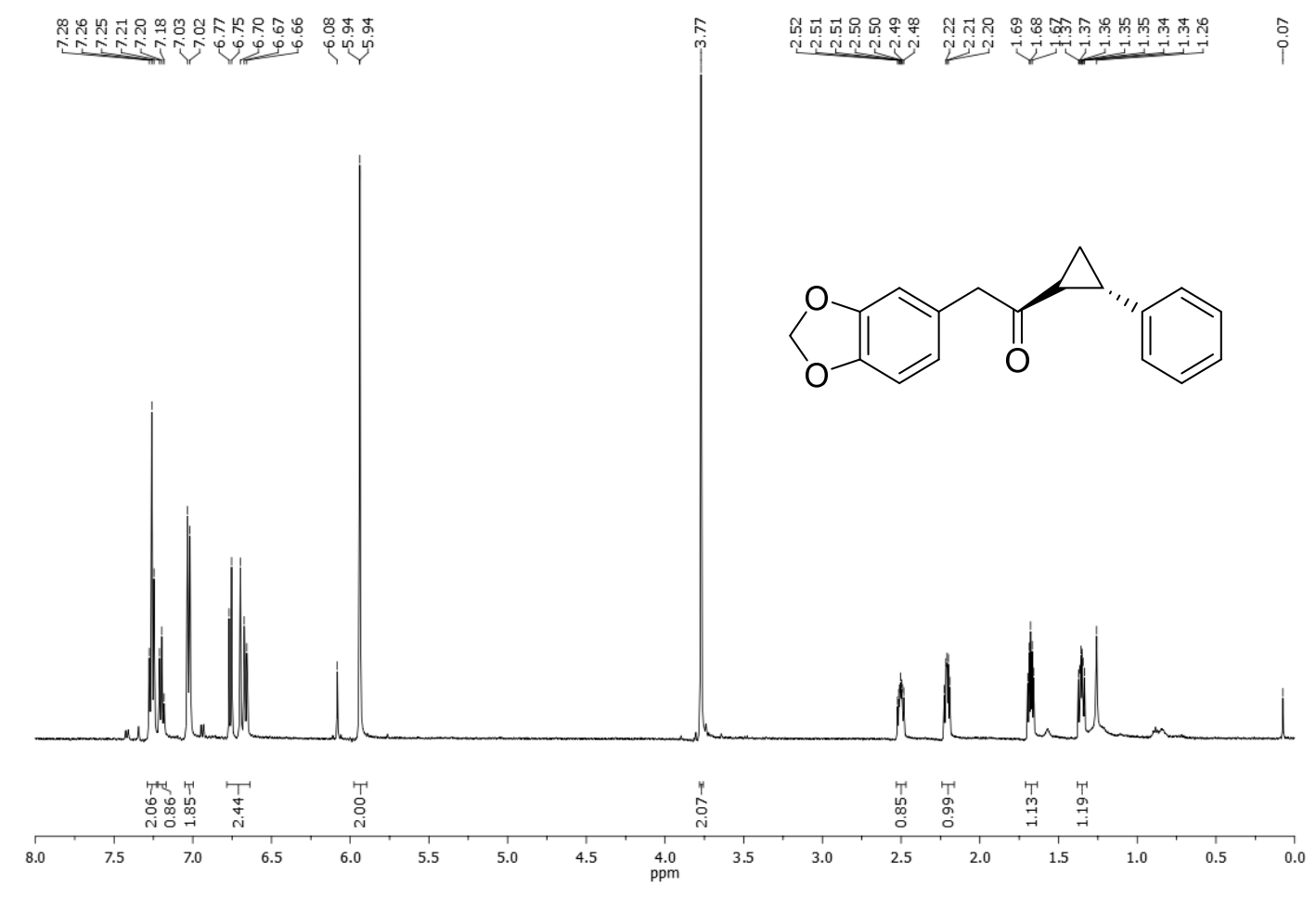

2-(benzo[1,3]dioxol-5-yl)-1-((1S, 2S)-2-phenylcyclopropyl)ethan-1-one (7h), ${ }^{13} \mathrm{C}$ NMR (126 $\left.\mathrm{MHz}, \mathrm{CDCl}_{3}\right)$ :

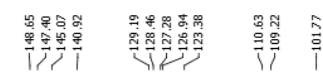<smiles>O=C(Cc1ccc2c(c1)OCO2)[C@H]1C[C@H]1c1ccccc1</smiles>

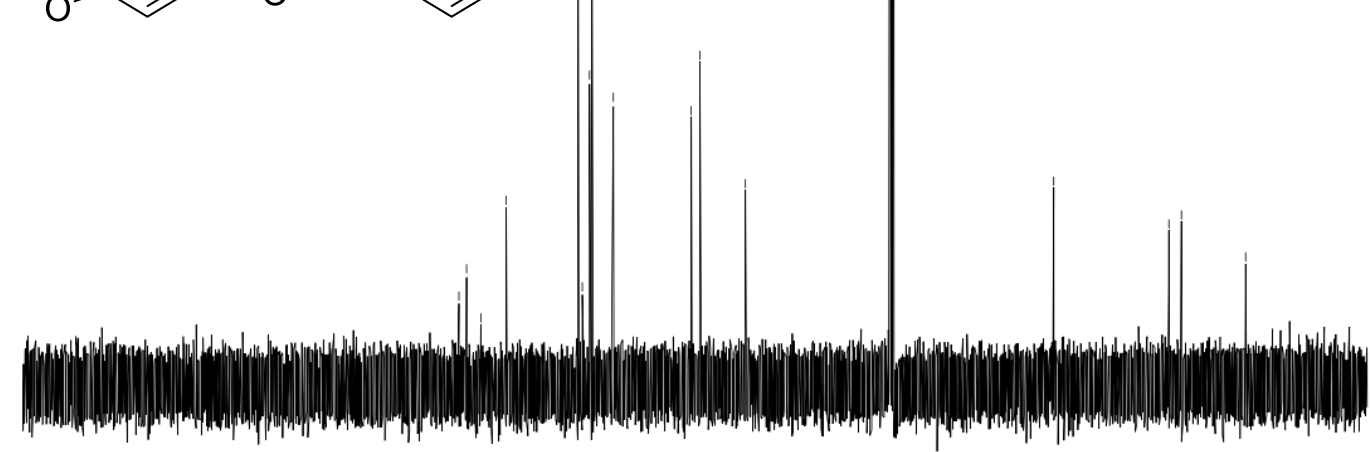

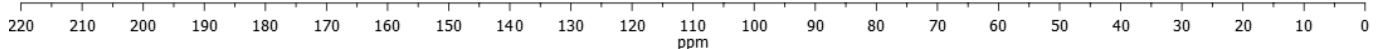


(S)-2-phenyl-1-((1S, 2S)-2-phenylcyclopropyl)propan-1-one (7i), ${ }^{1} \mathrm{H}$ NMR (500 MHz, $\left.\mathrm{CDCl}_{3}\right)$ :

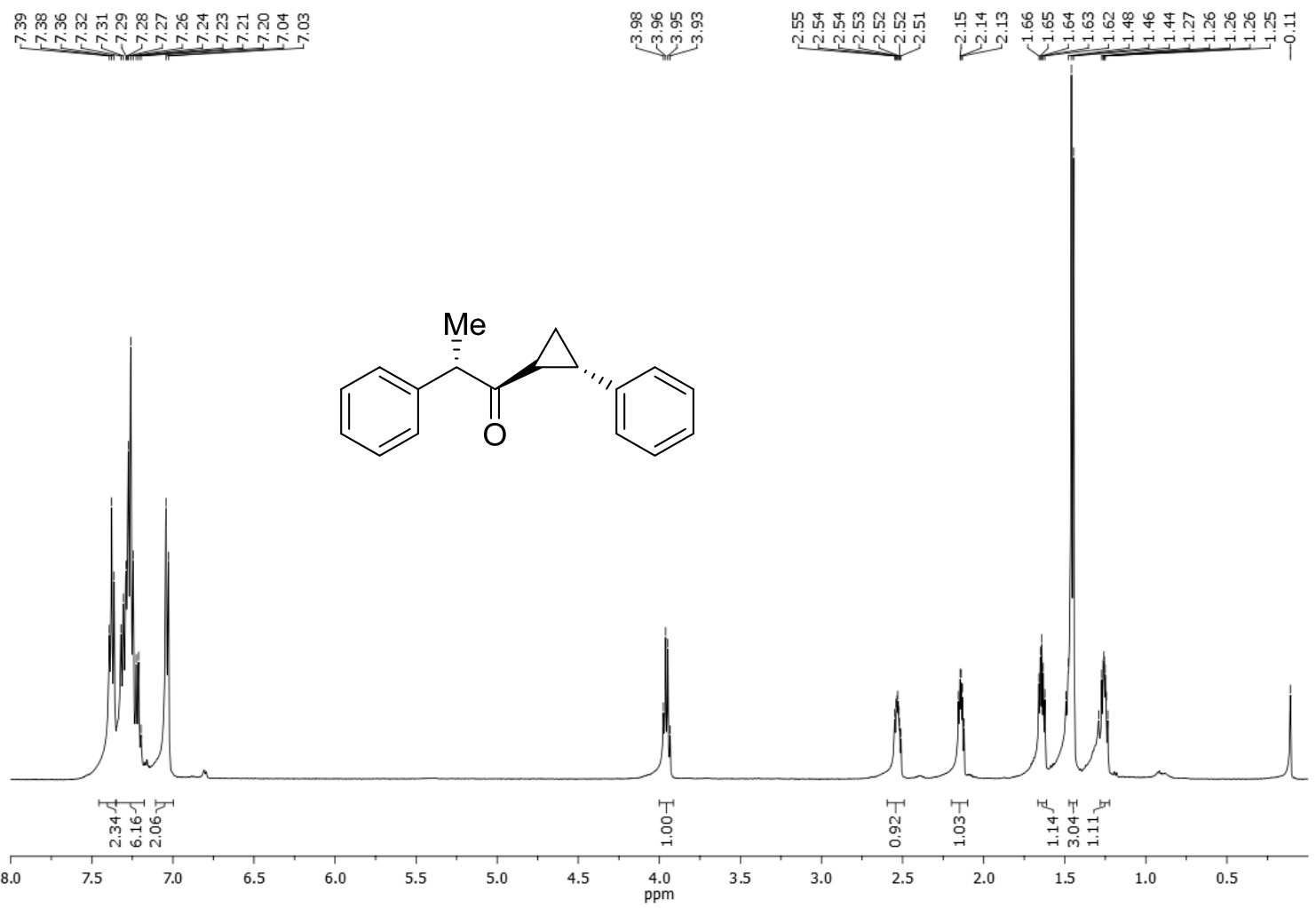

(S)-2-phenyl-1-((1S, 2S)-2-phenylcyclopropyl)propan-1-one (7i), $\left.{ }^{13} \mathrm{C} \mathrm{NMR} \mathrm{(126} \mathrm{MHz,} \mathrm{CDCl}_{3}\right)$ :

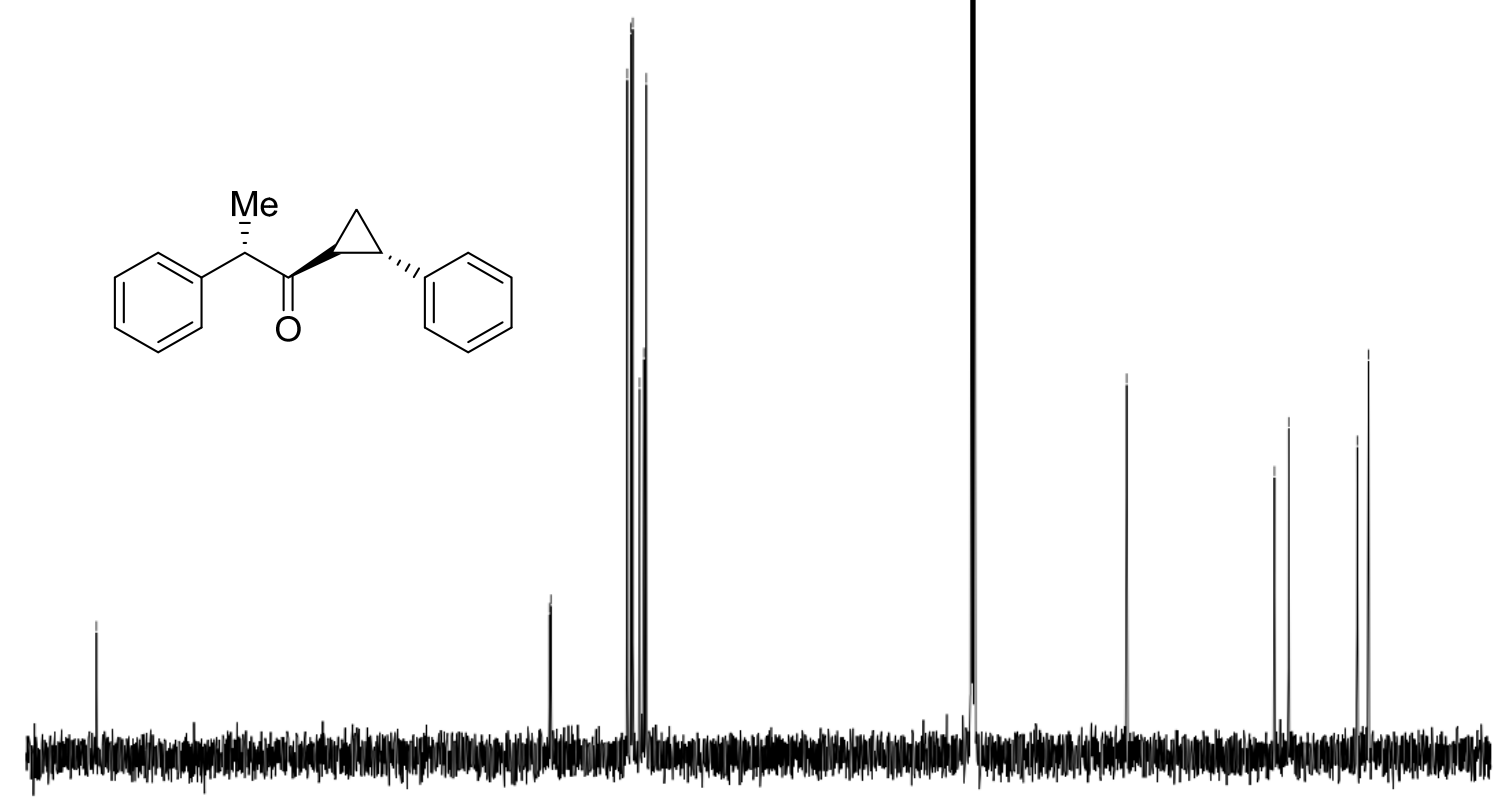

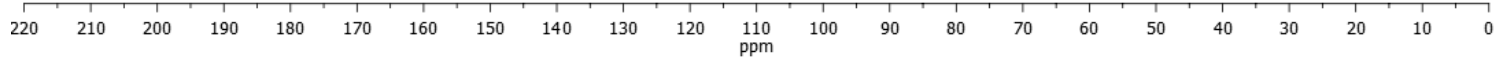


2-cyclohexyl-1-((1S,2S)-2-phenylcyclopropyl)ethan-1-one (7k), ${ }^{1} \mathrm{H}$ NMR (500 MHz, $\left.\mathrm{CDCl}_{3}\right)$ :

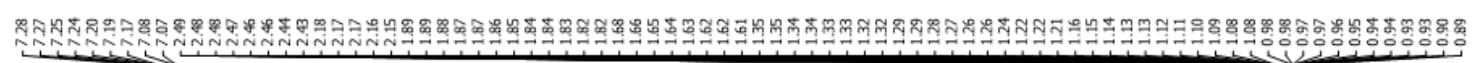

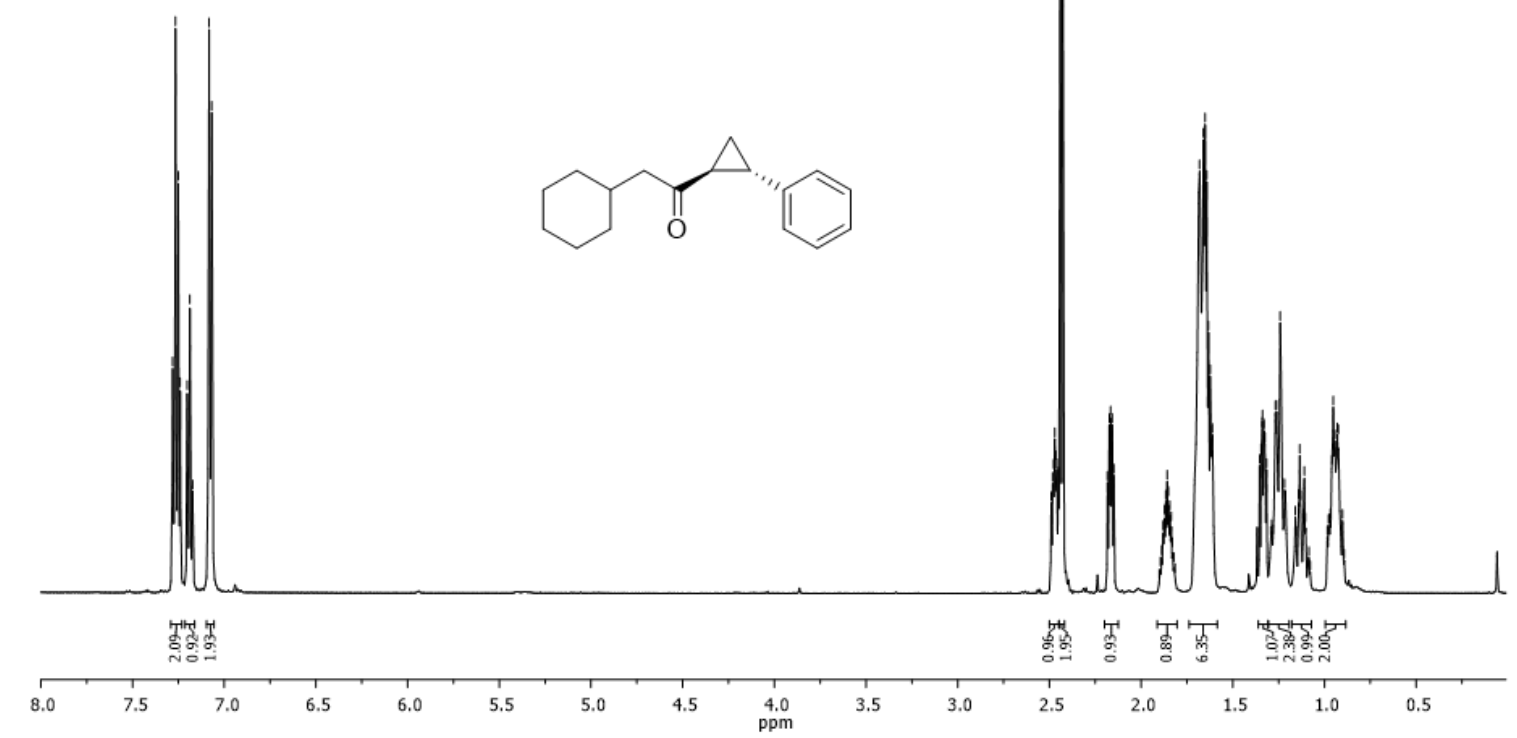

2-cyclohexyl-1-((1S,2S)-2-phenylcyclopropyl)ethan-1-one (7k), ${ }^{13} \mathrm{C}$ NMR (126 MHz, $\left.\mathrm{CDCl}_{3}\right)$ :

蕰

岱
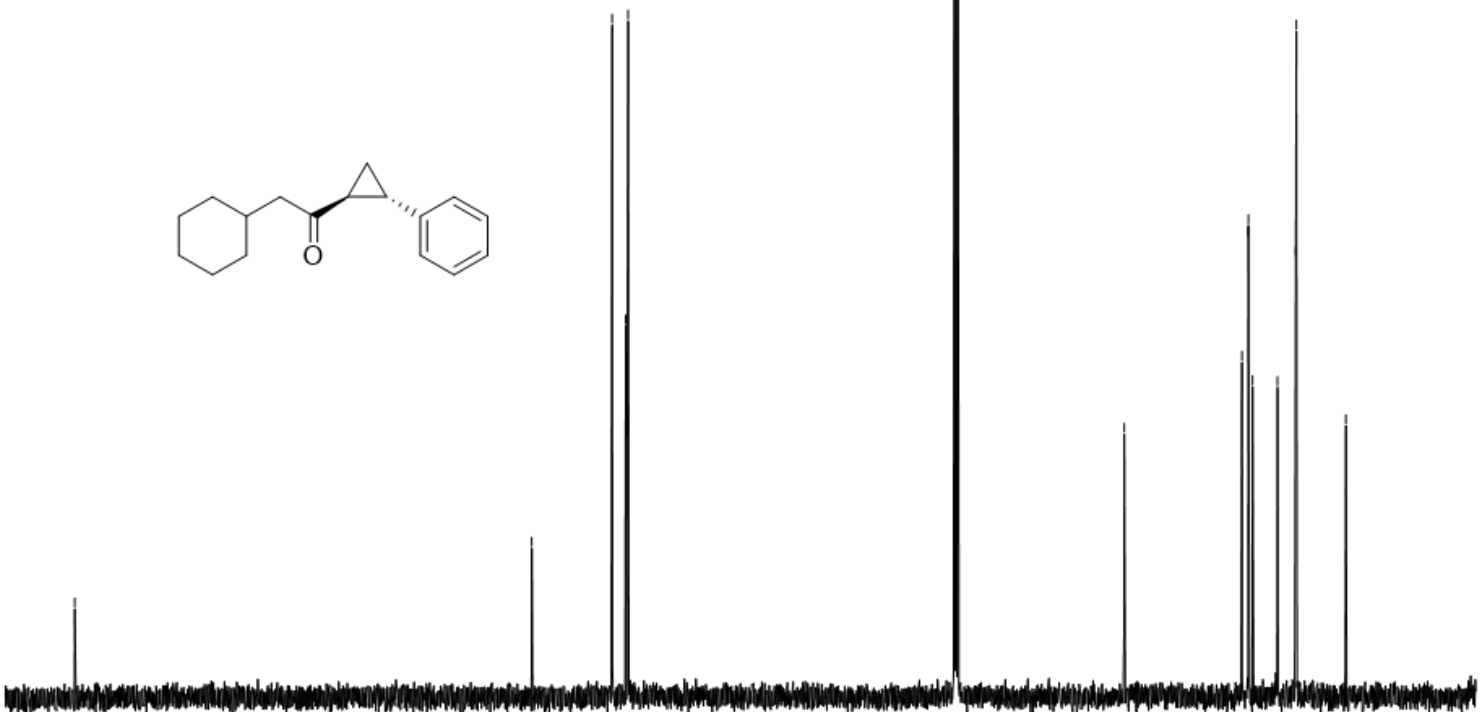

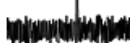

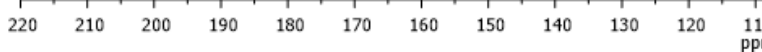


2-cyclopropyl-1-((1S,2S)-2-phenylcyclopropyl)ethan-1-one (7l), ${ }^{1} \mathrm{H}$ NMR (500 MHz, $\left.\mathrm{CDCl}_{3}\right)$ :

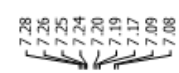

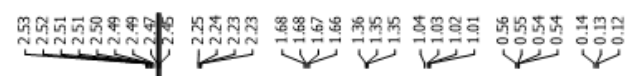

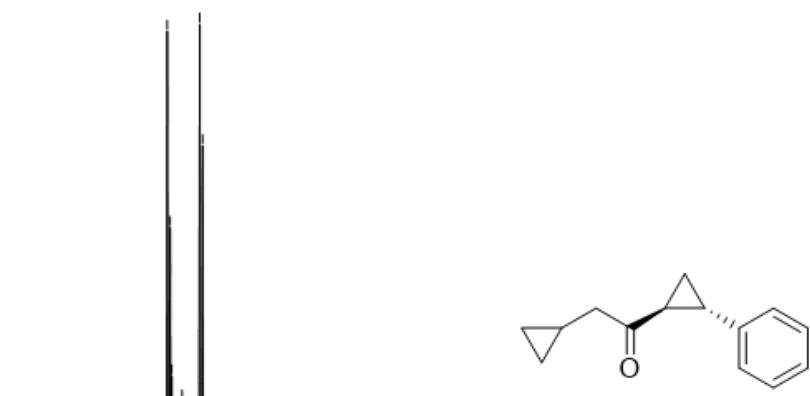

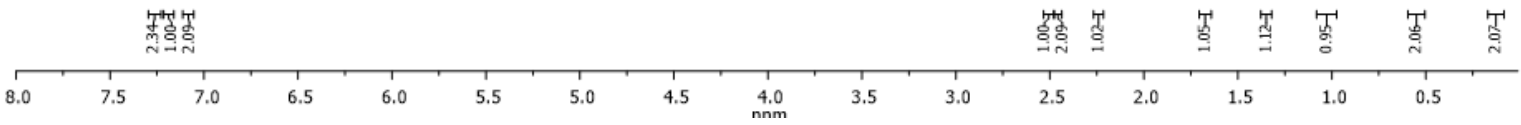

2-cyclopropyl-1-((1S,2S)-2-phenylcyclopropyl)ethan-1-one (7l), ${ }^{13} \mathrm{C} \mathrm{NMR}\left(126 \mathrm{MHz}, \mathrm{CDCl}_{3}\right)$ :

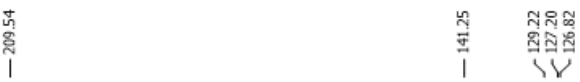

i

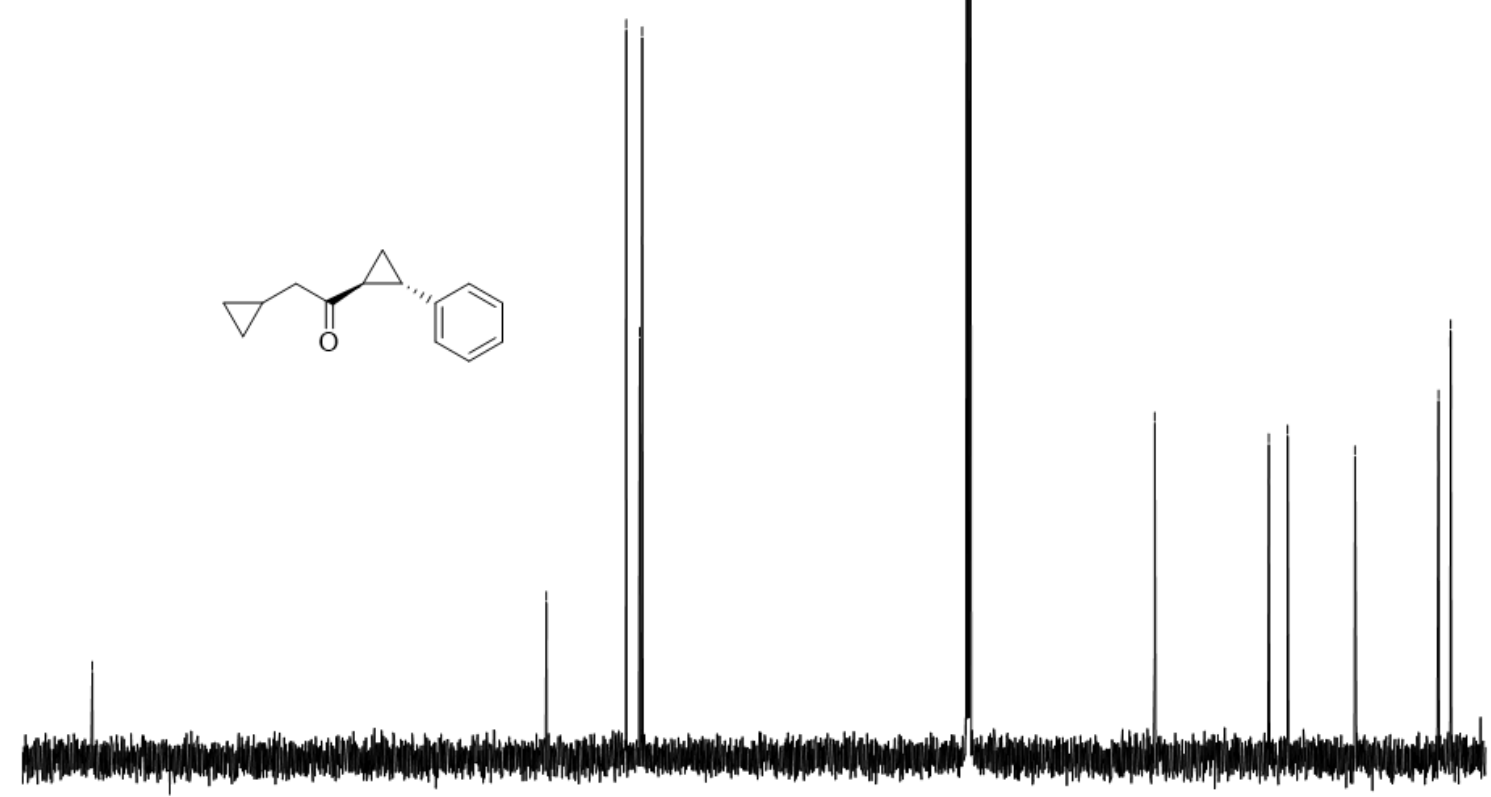

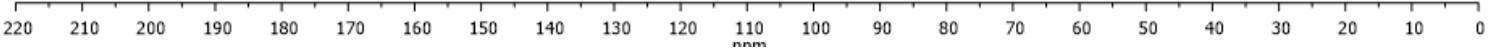


4-methyl-1-((1S,2S)-2-phenylcyclopropyl)pentan-1-one (7m), ${ }^{1} \mathrm{H}$ NMR (500 MHz, $\left.\mathrm{CDCl}_{3}\right)$ :

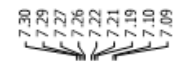

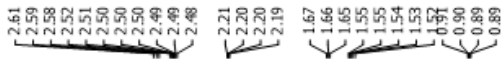
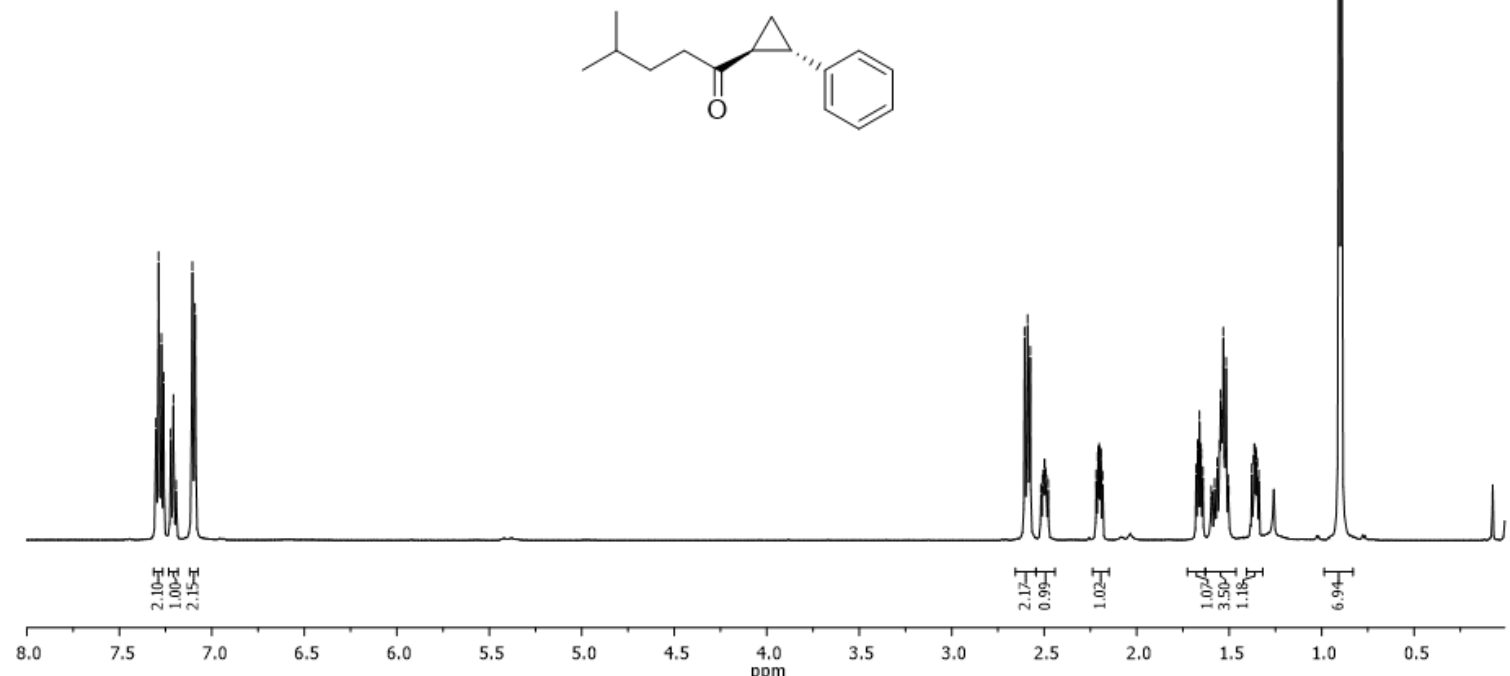

4-methyl-1-((1S,2S)-2-phenylcyclopropyl)pentan-1-one (7m), ${ }^{13} \mathrm{C}$ NMR (126 MHz, $\left.\mathrm{CDCl}_{3}\right)$ :

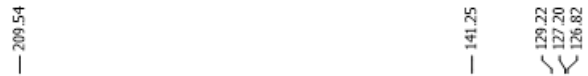
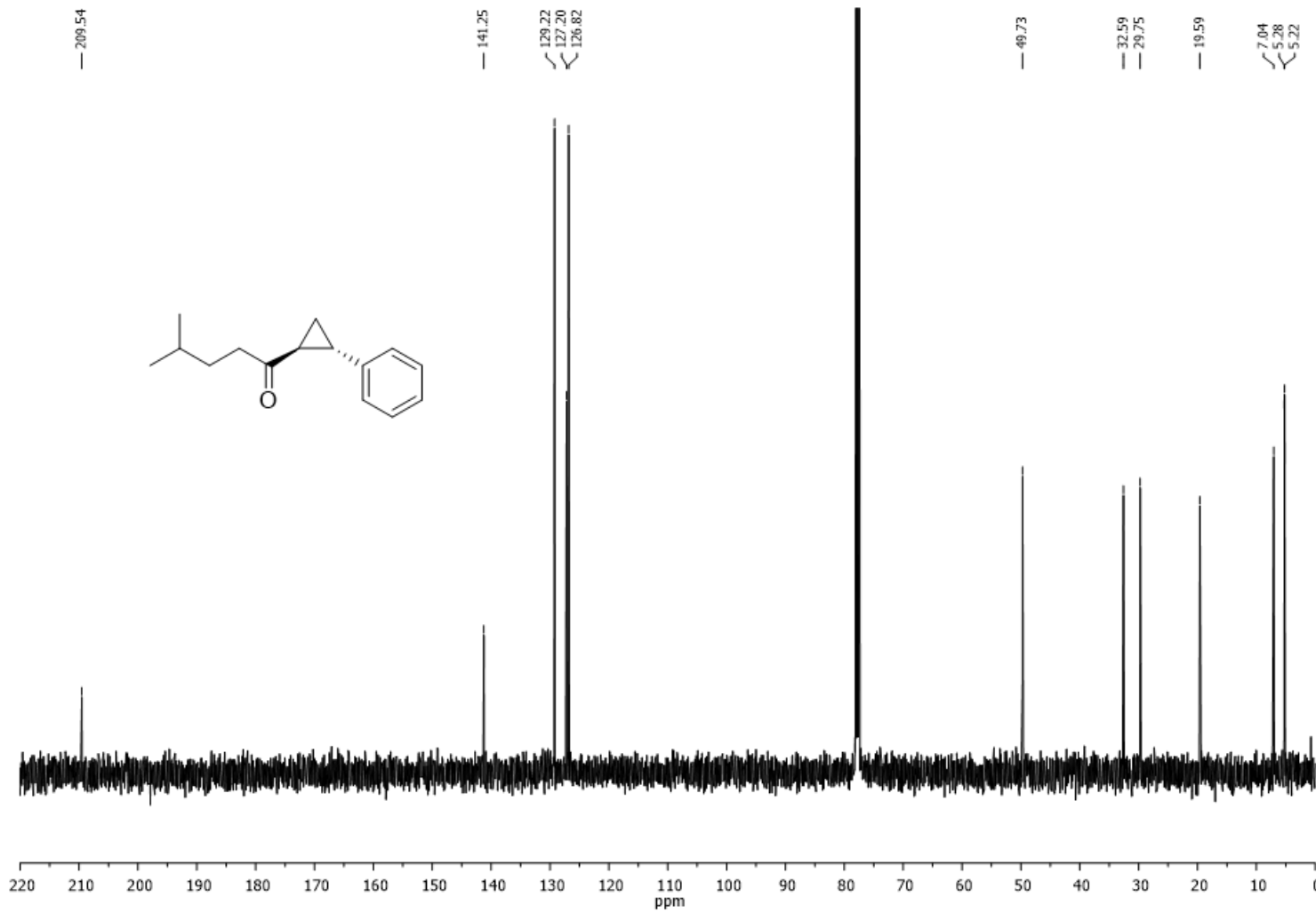
3-methyl-1-((1S,2S)-2-phenylcyclopropyl)butan-1-one (7n), ${ }^{1} \mathrm{H}$ NMR (500 MHz, $\left.\mathrm{CDCl}_{3}\right)$ :
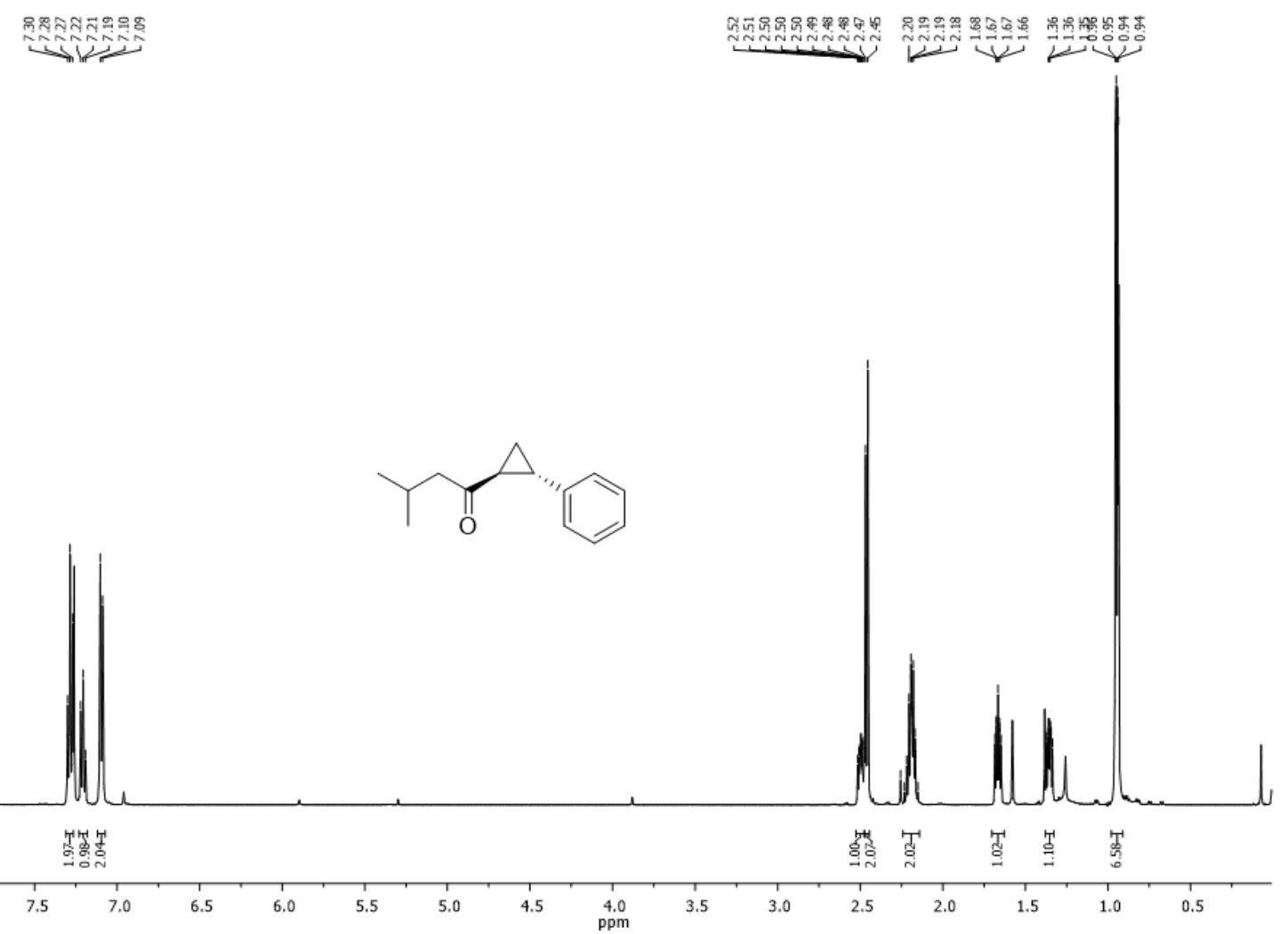

3-methyl-1-((1S,2S)-2-phenylcyclopropyl)butan-1-one (7n), ${ }^{13} \mathrm{C}$ NMR (126 MHz, $\left.\mathrm{CDCl}_{3}\right)$ :

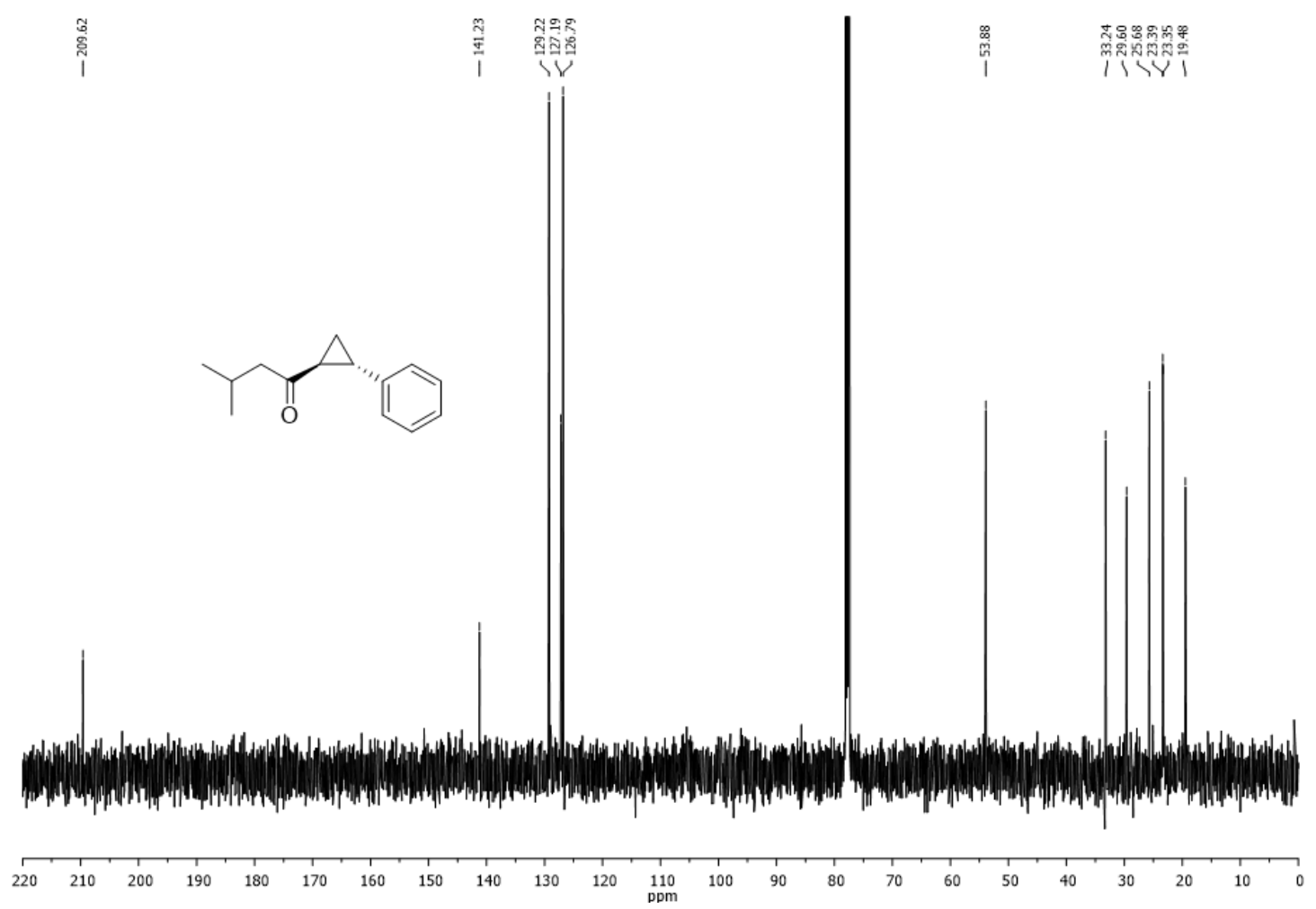


2-(p-tolyl)-1-((1S,2S)-2-(p-tolyl)cyclopropyl)ethan-1-one (8a), ${ }^{1} \mathrm{H}$ NMR (500 MHz, $\left.\mathrm{CDCl}_{3}\right)$ :
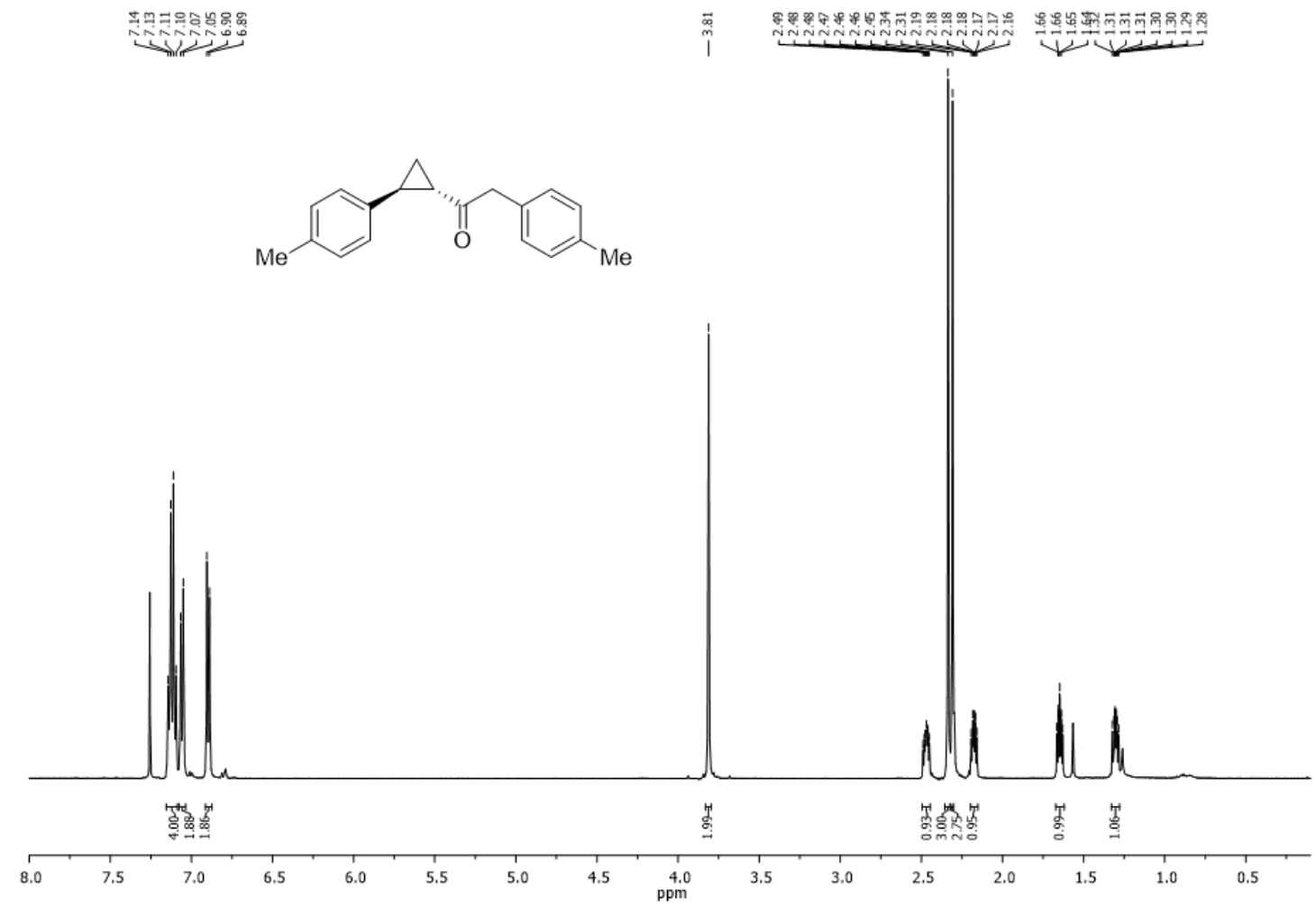

2-(p-tolyl)-1-((1S,2S)-2-(p-tolyl)cyclopropyl)ethan-1-one (8a), ${ }^{13} \mathrm{C}$ NMR $\left(126 \mathrm{MHz}, \mathrm{CDCl}_{3}\right)$ :

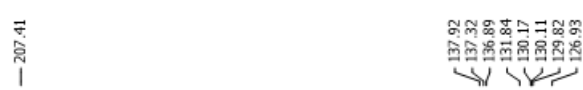

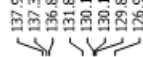

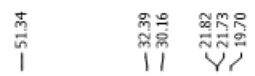<smiles>Cc1ccc(CC(=O)Cc2ccc([N+](=O)[O-])cc2)cc1</smiles>

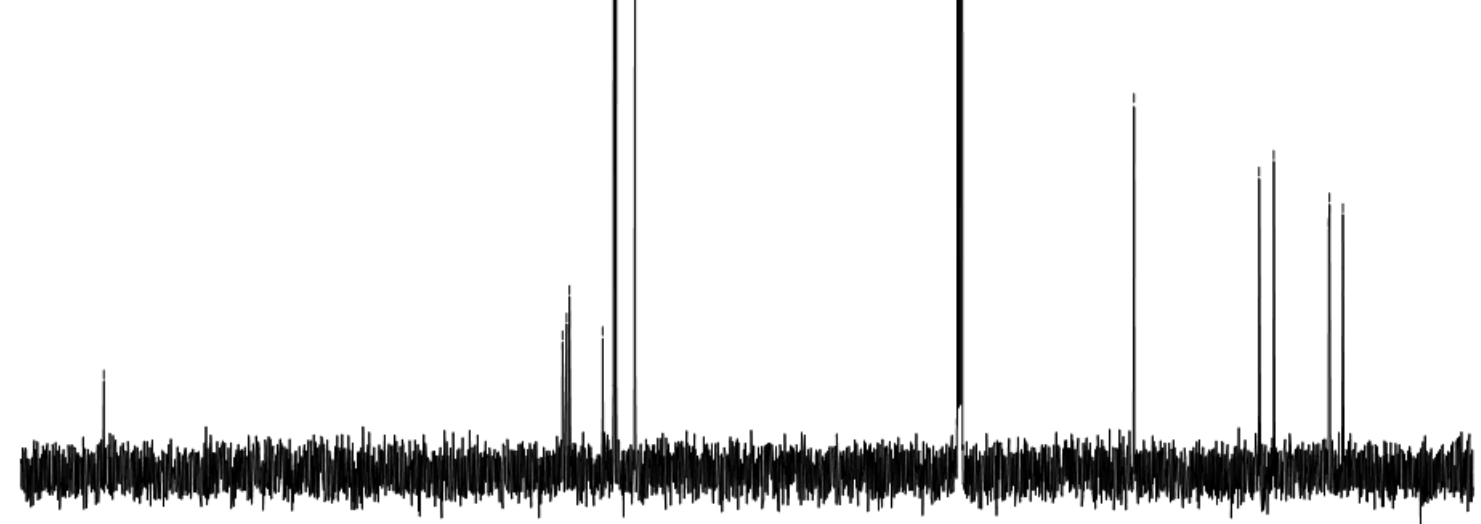

$\begin{array}{llllllllllll}220 & 210 & 200 & 190 & 180 & 170 & 160 & 150 & 140 & 130 & 120 & 110\end{array}$ 
1-((1S,2S)-2-(4-methoxyphenyl)cyclopropyl)-2-(p-tolyl)ethan-1-one (8b), ${ }^{1} \mathrm{H}$ NMR (500 MHz, $\left.\mathrm{CDCl}_{3}\right)$ :

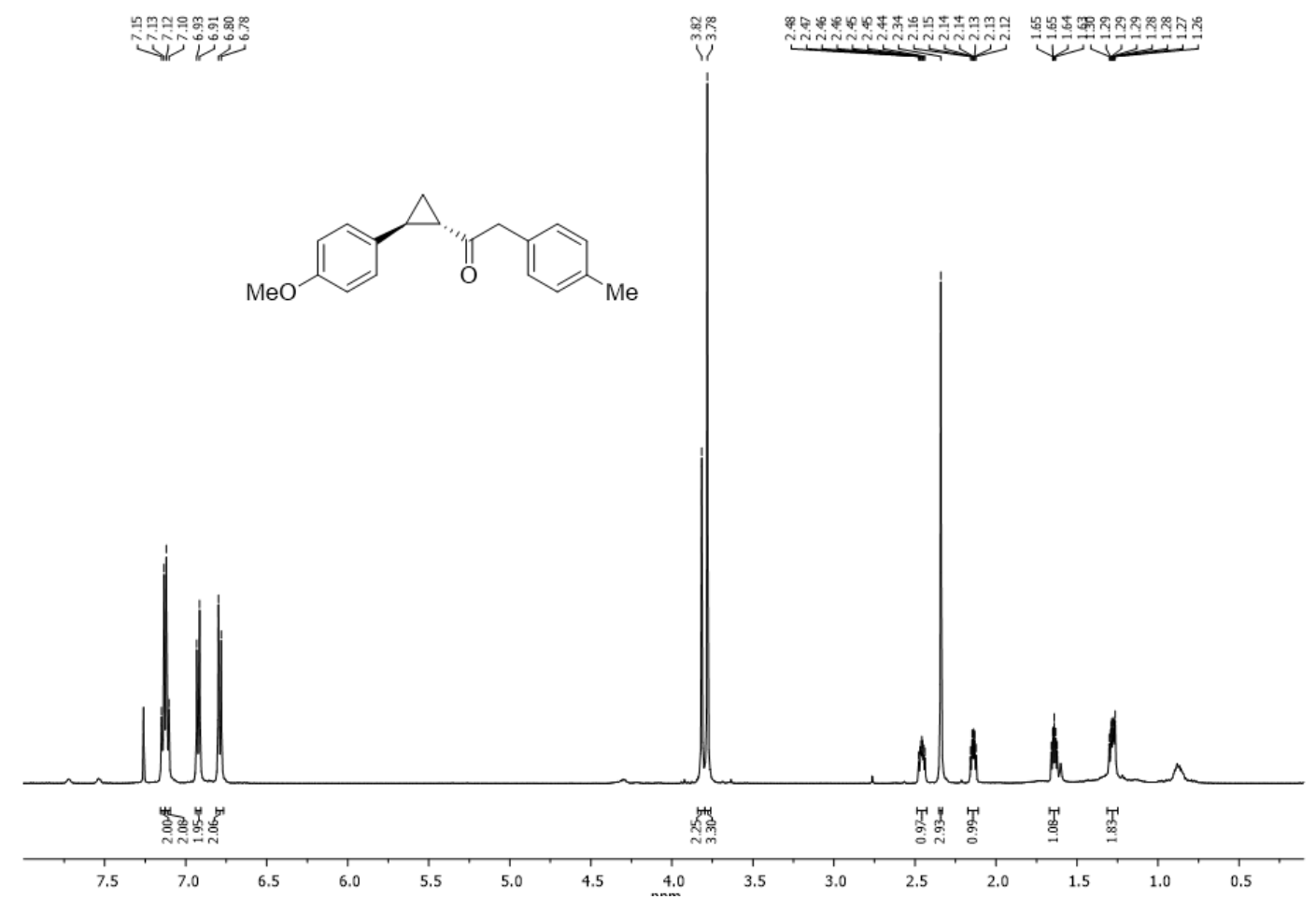

1-((1S,2S)-2-(4-methoxyphenyl)cyclopropyl)-2-(p-tolyl)ethan-1-one (8b), ${ }^{13} \mathrm{C}$ NMR (126 MHz, $\left.\mathrm{CDCl}_{3}\right)$ :
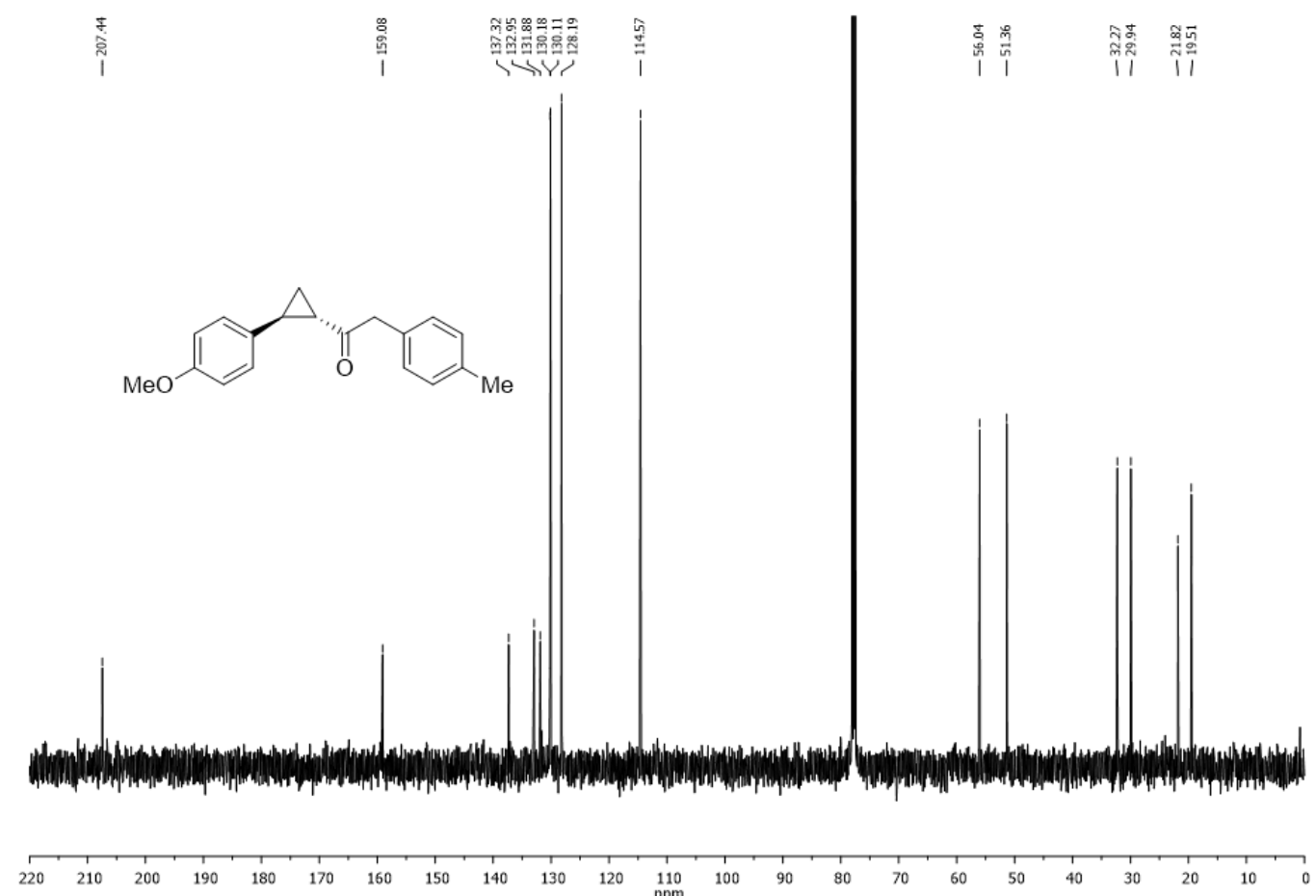
1-((1S,2S)-2-(4-methoxyphenyl)cyclopropyl)-2-(p-tolyl)ethan-1-one (8c), ${ }^{1} \mathrm{H}$ NMR (500 MHz, $\left.\mathrm{CDCl}_{3}\right)$ :

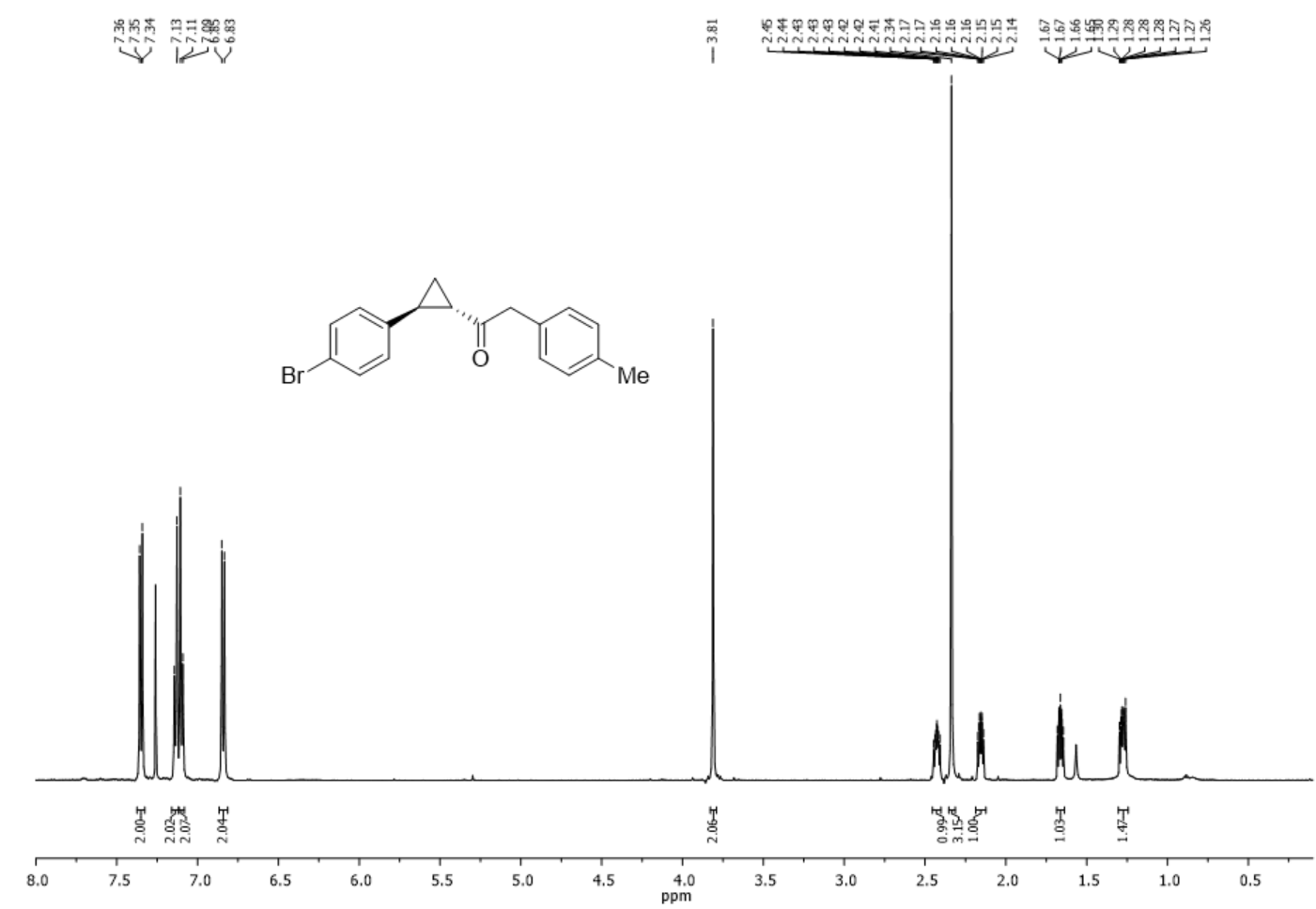

1-((1S,2S)-2-(4-methoxyphenyl)cyclopropyl)-2-(p-tolyl)ethan-1-one (8c), ${ }^{13} \mathrm{C}$ NMR (126 MHz, $\left.\mathrm{CDCl}_{3}\right)$ :
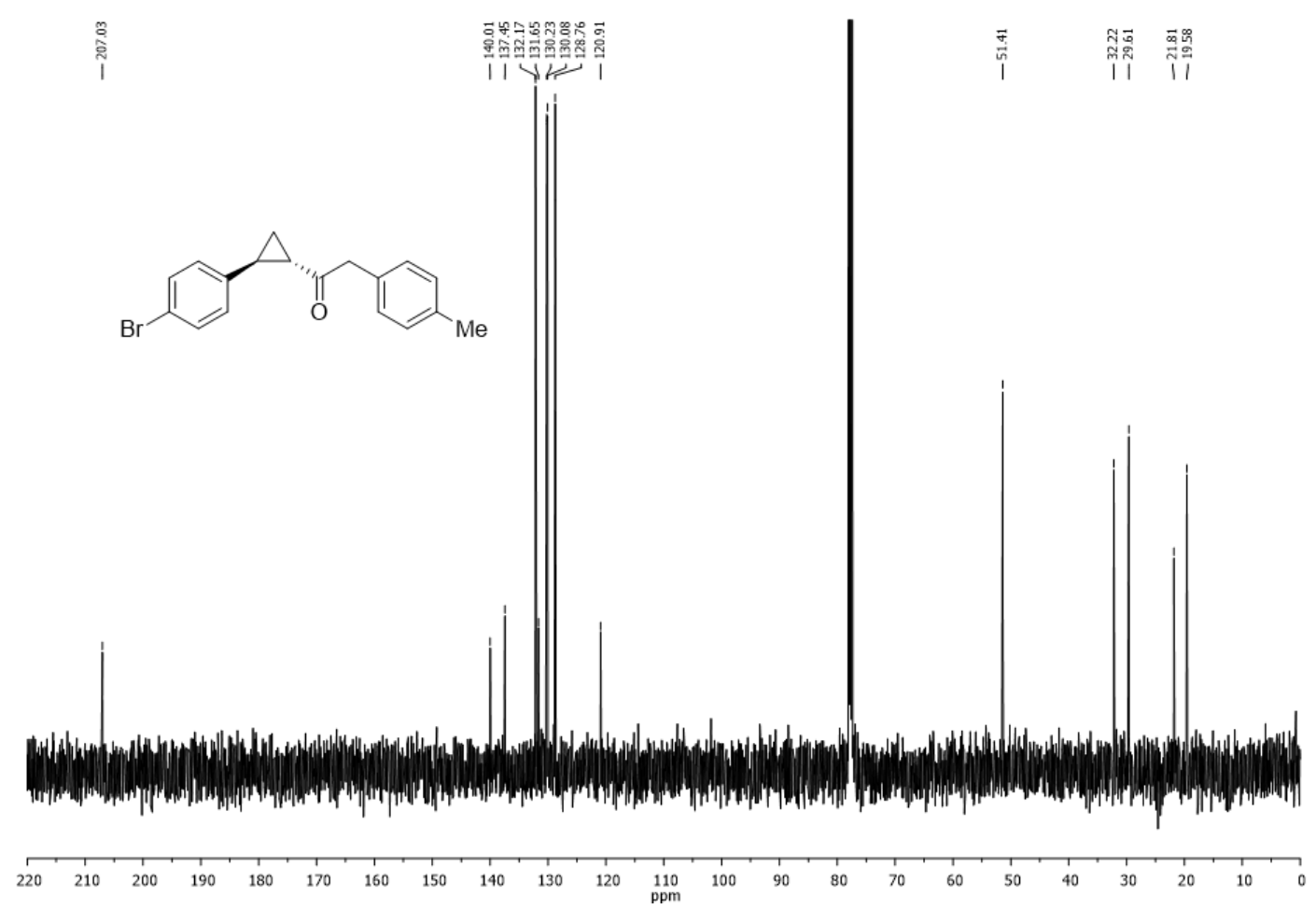
2-phenyl-1-((1S,2S)-2-phenylcyclopropyl)ethan-1-ol (9), ${ }^{1} \mathrm{H} \quad \mathrm{NMR} \quad\left(400 \quad \mathrm{MHz}, \quad \mathrm{CDCl}_{3}\right)$ :

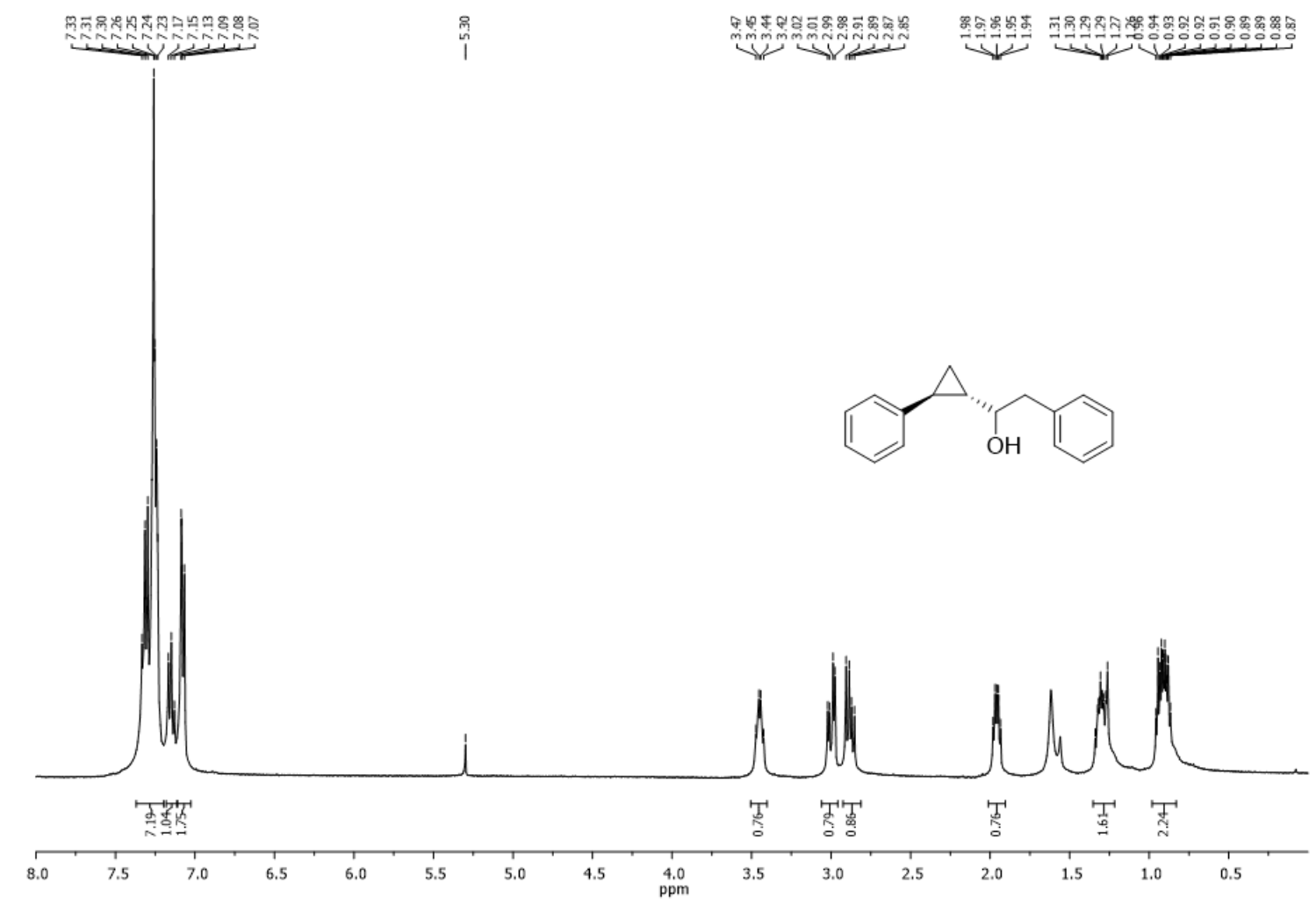

2-phenyl-1-((1S,2S)-2-phenylcyclopropyl)ethan-1-ol (9), ${ }^{13} \mathrm{C}$ NMR (101 MHz, $\left.\mathrm{CDCl}_{3}\right)$ :

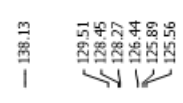
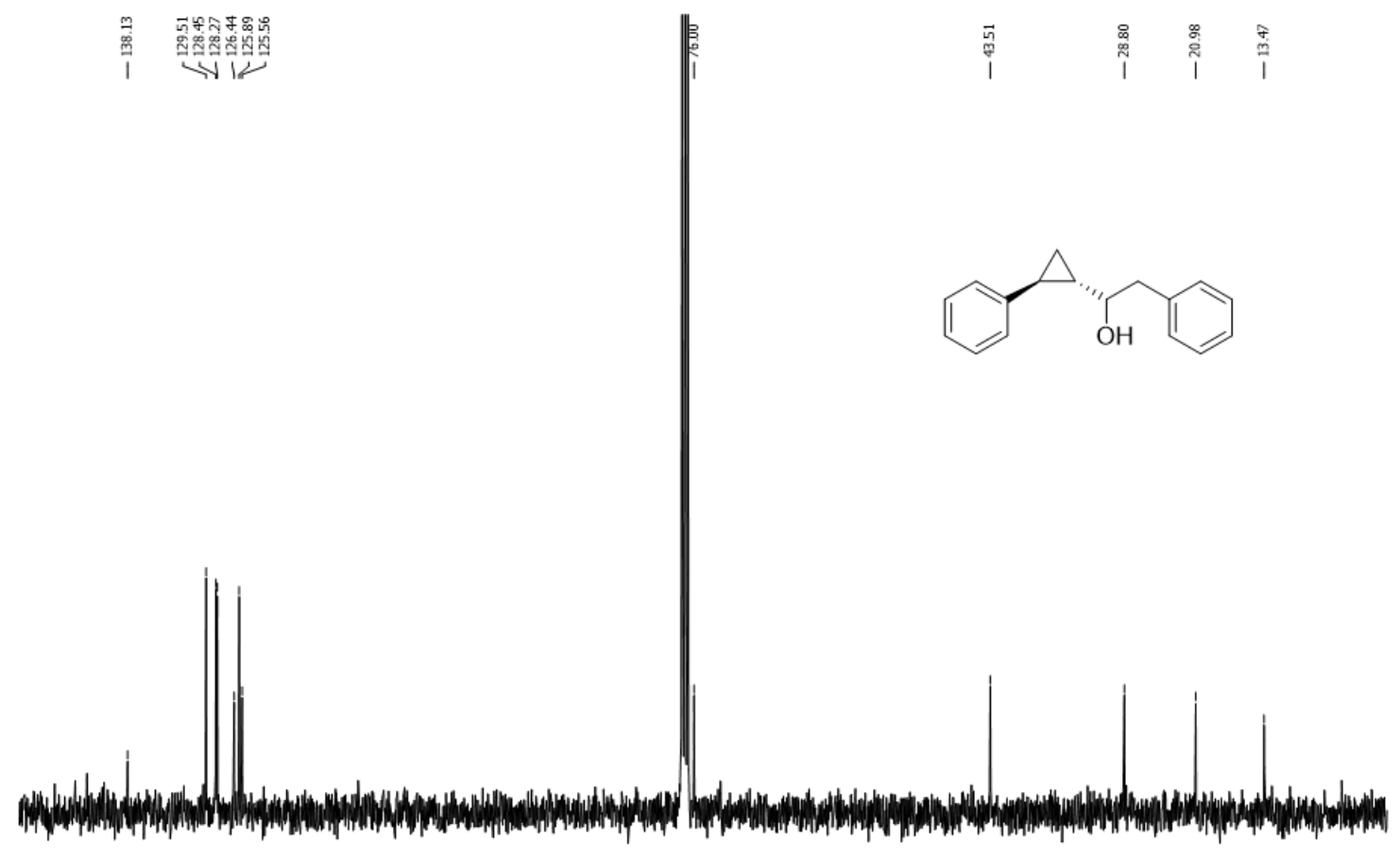

$\begin{array}{lllllllllllllllllllllllllllllllllllll}145 & 140 & 135 & 130 & 125 & 120 & 115 & 110 & 105 & 100 & 95 & 90 & 85 & 80 & 75 & 70 & 65 & 60 & 55 & 50 & 45 & 40 & 35 & 30 & 25 & 20 & 15 & 10 & 5 & 0\end{array}$ 
1,2-diphenyl-1-((1S,2S)-2-phenylcyclopropyl)ethan-1-ol (10), ${ }^{1} \mathrm{H}$ NMR (400 MHz, $\left.\mathrm{CDCl}_{3}\right)$ :

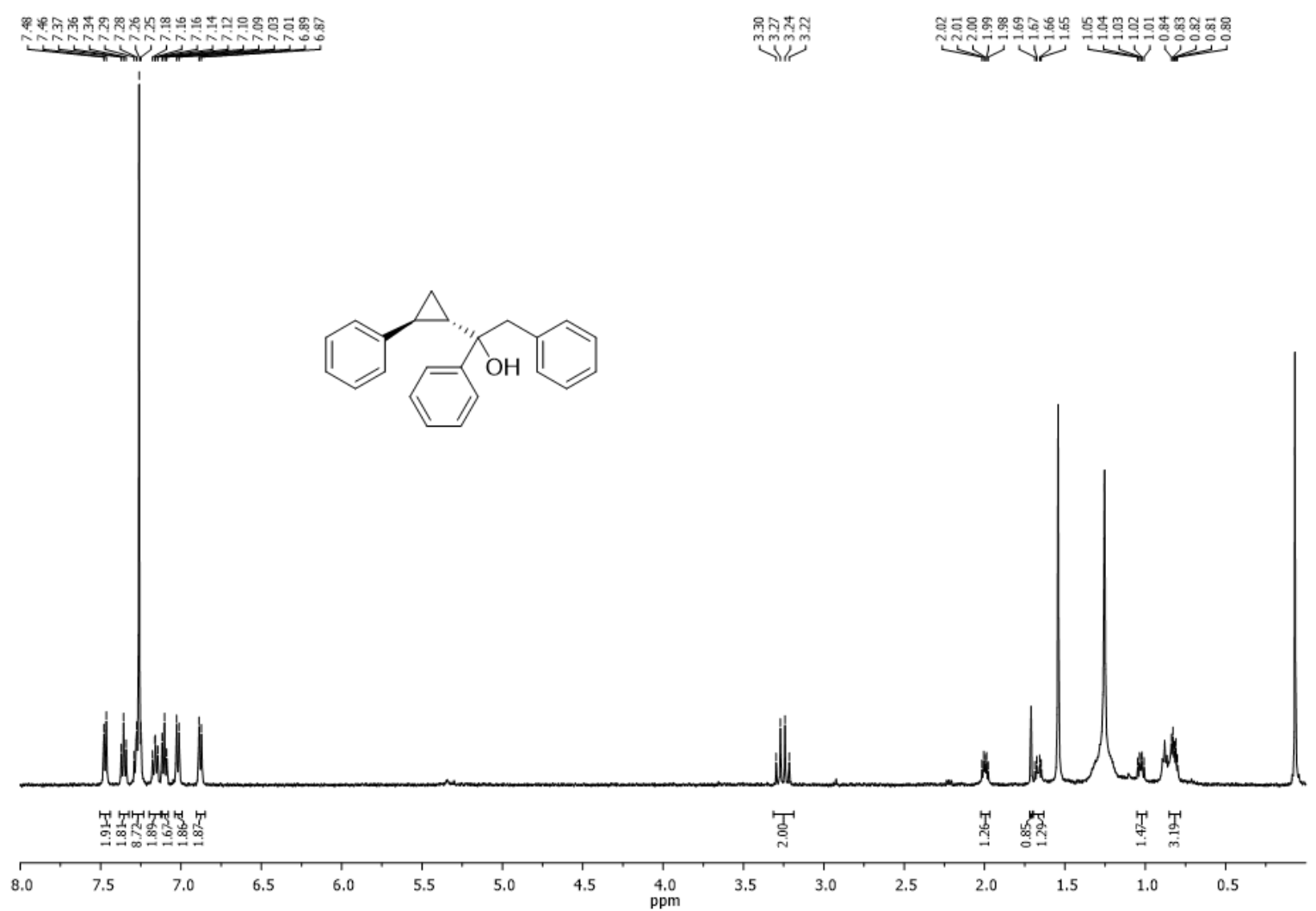

1,2-diphenyl-1-((1S,2S)-2-phenylcyclopropyl)ethan-1-ol (10), ${ }^{13} \mathrm{C} \mathrm{NMR}\left(101 \mathrm{MHz}, \mathrm{CDCl}_{3}\right)$ :

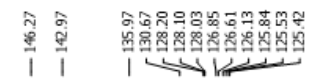

i j
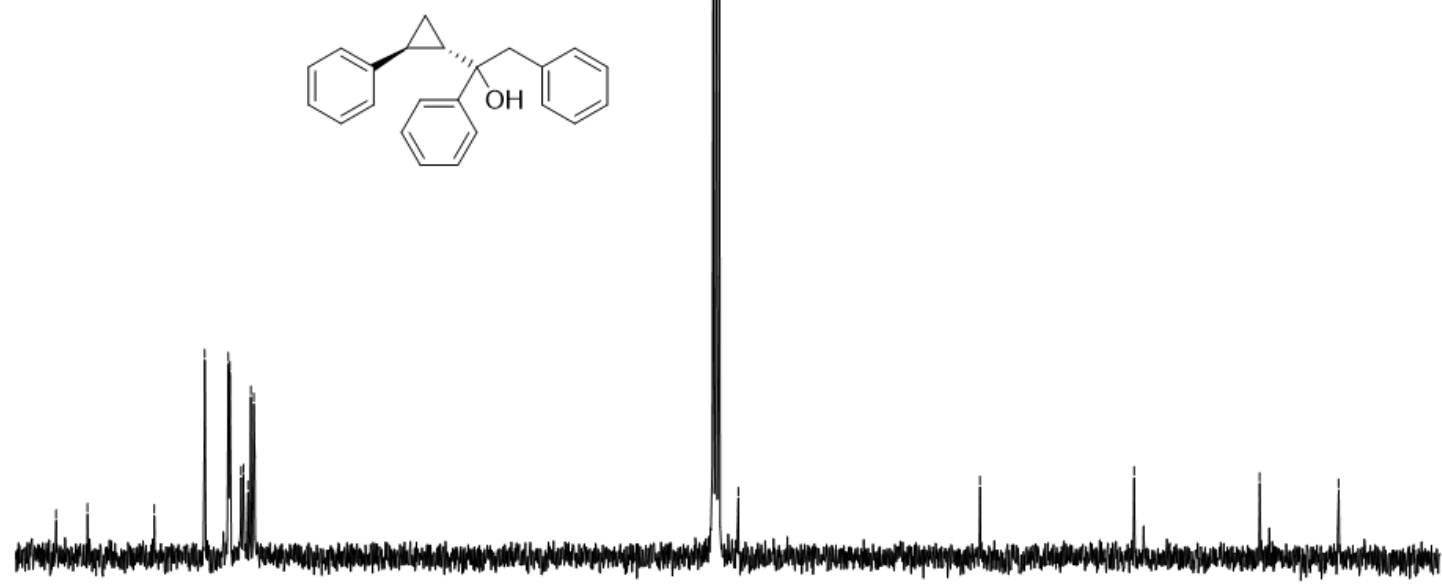

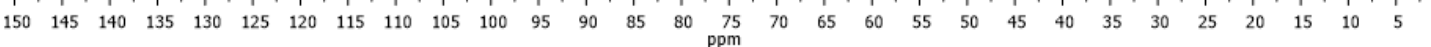


(2-fluoro-2-((1S,2S)-2-phenylcyclopropyl)ethyl)benzene (11), ${ }^{1} \mathrm{H} \mathrm{NMR}\left(500 \mathrm{MHz}, \mathrm{CDCl}_{3}\right)$ :

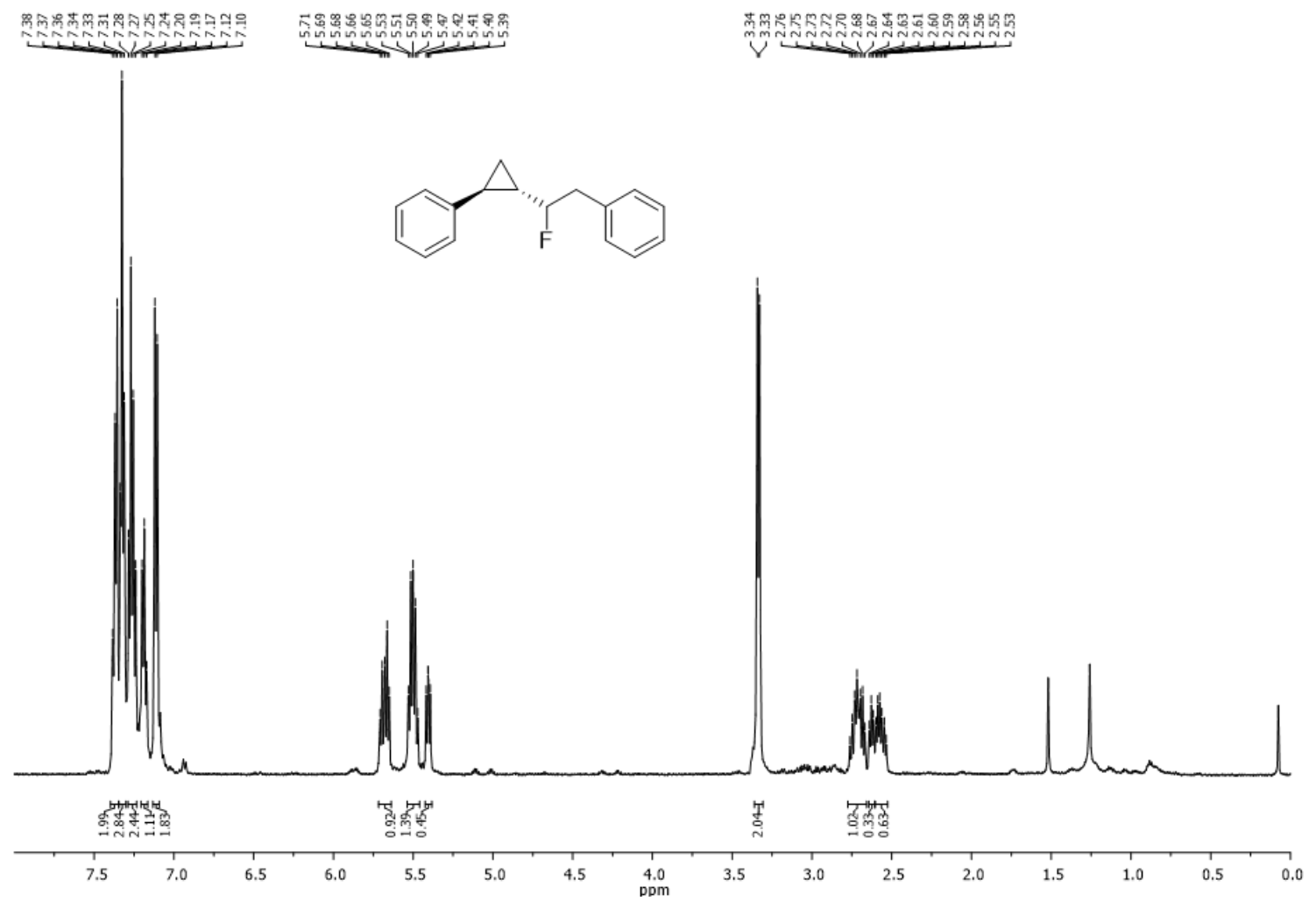

(2-fluoro-2-((1S,2S)-2-phenylcyclopropyl)ethyl)benzene (11), $\left.{ }^{13} \mathrm{C} \mathrm{NMR} \mathrm{(126} \mathrm{MHz,} \mathrm{CDCl}_{3}\right)$ :

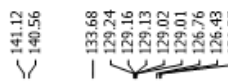

影恙

羿䒁
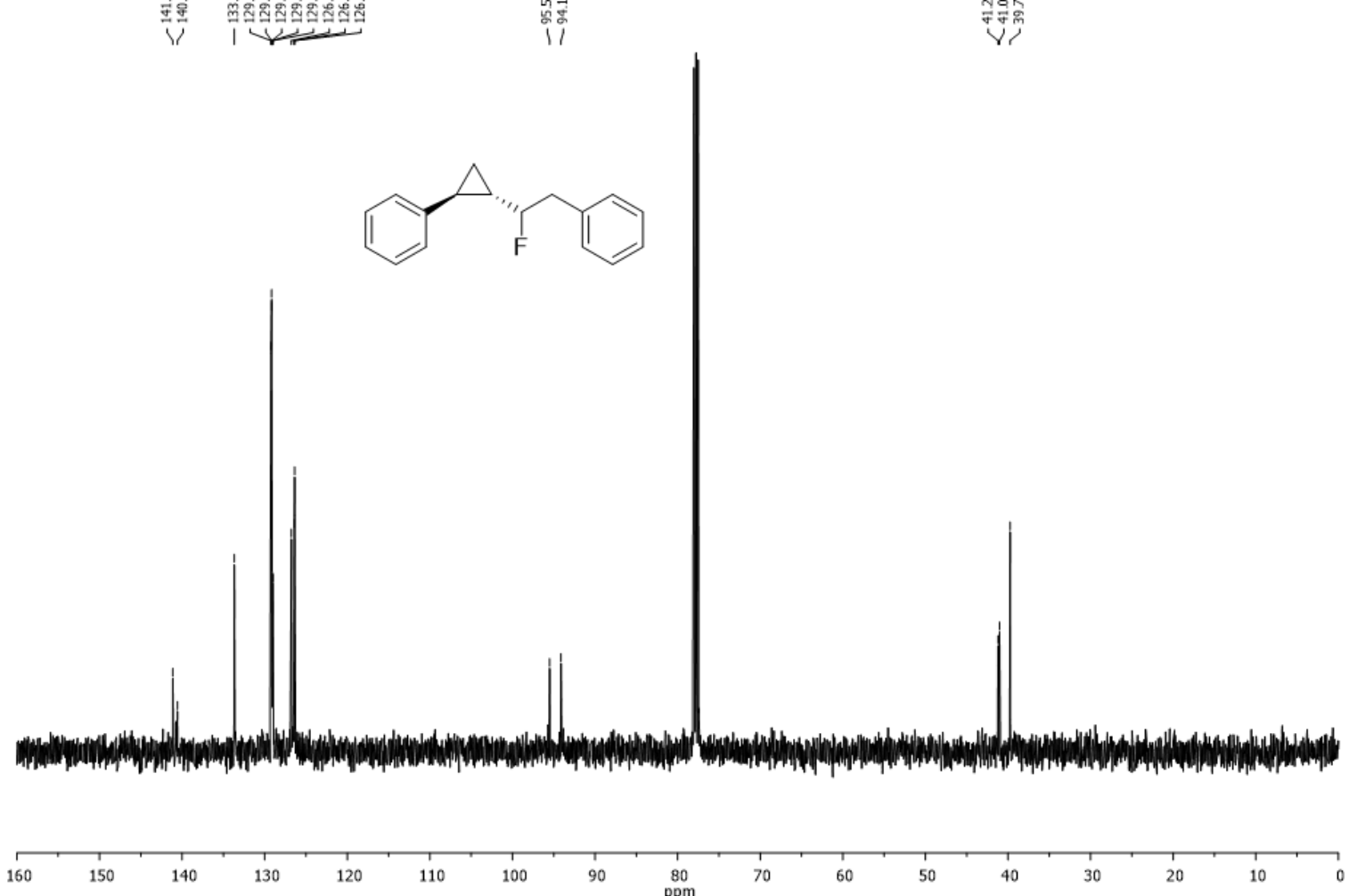
(2-fluoro-2-((IS,2S)-2-phenylcyclopropyl)ethyl)benzene (11), ${ }^{19} \mathrm{~F}$ NMR (376 MHz, $\left.\mathrm{CDCl}_{3}\right)$ :
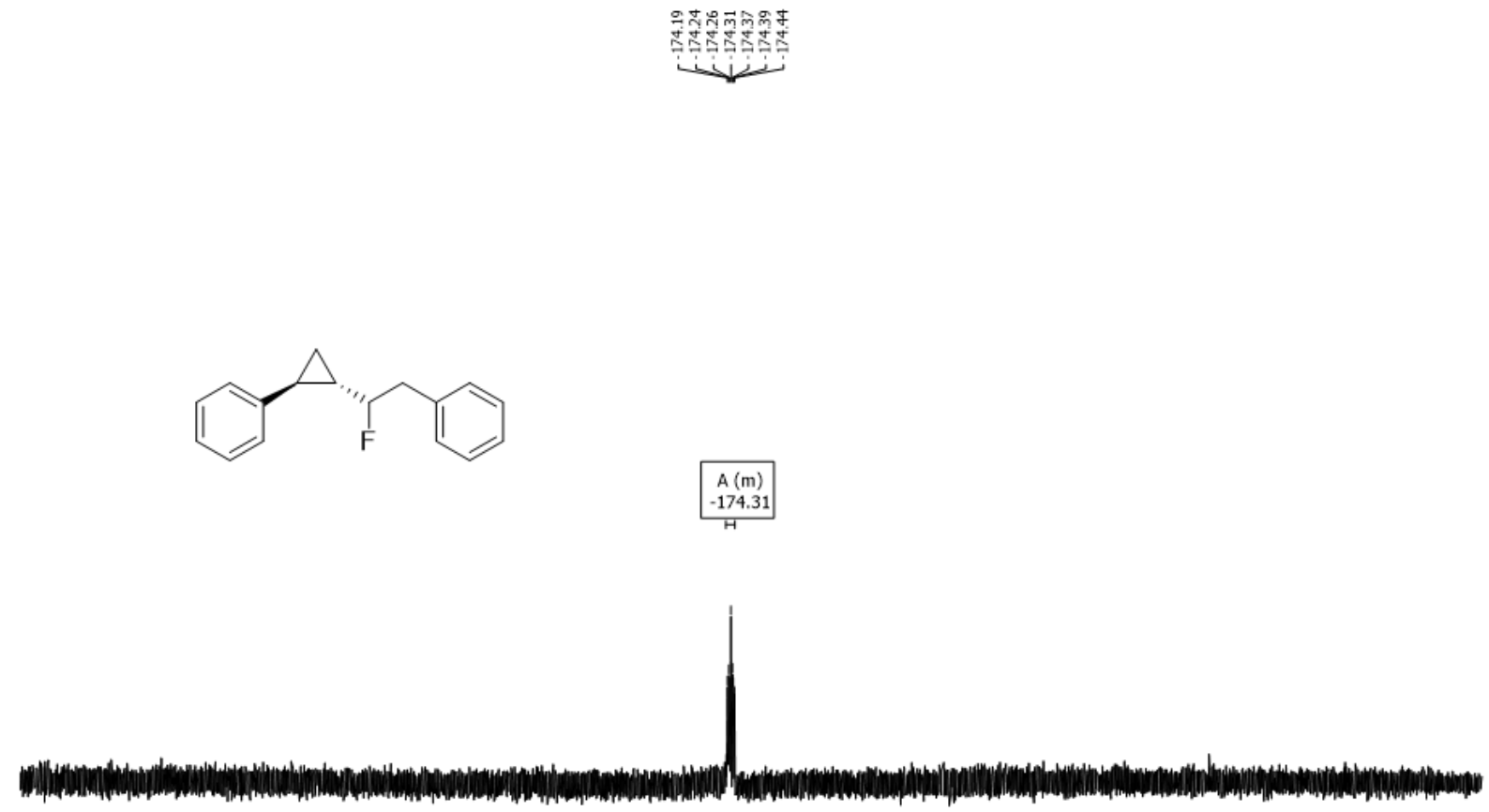

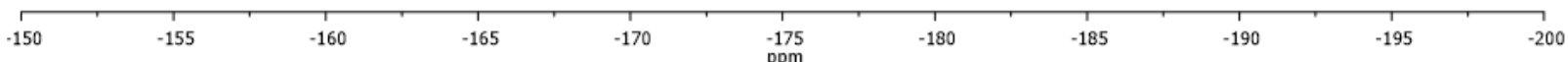


$N$-((2-phenyl-1-((1S,2S)-2-phenylcyclopropyl)ethyl)aniline (12), ${ }^{1} \mathrm{H}$ NMR $\left(500 \mathrm{MHz}, \mathrm{CDCl}_{3}\right)$ :

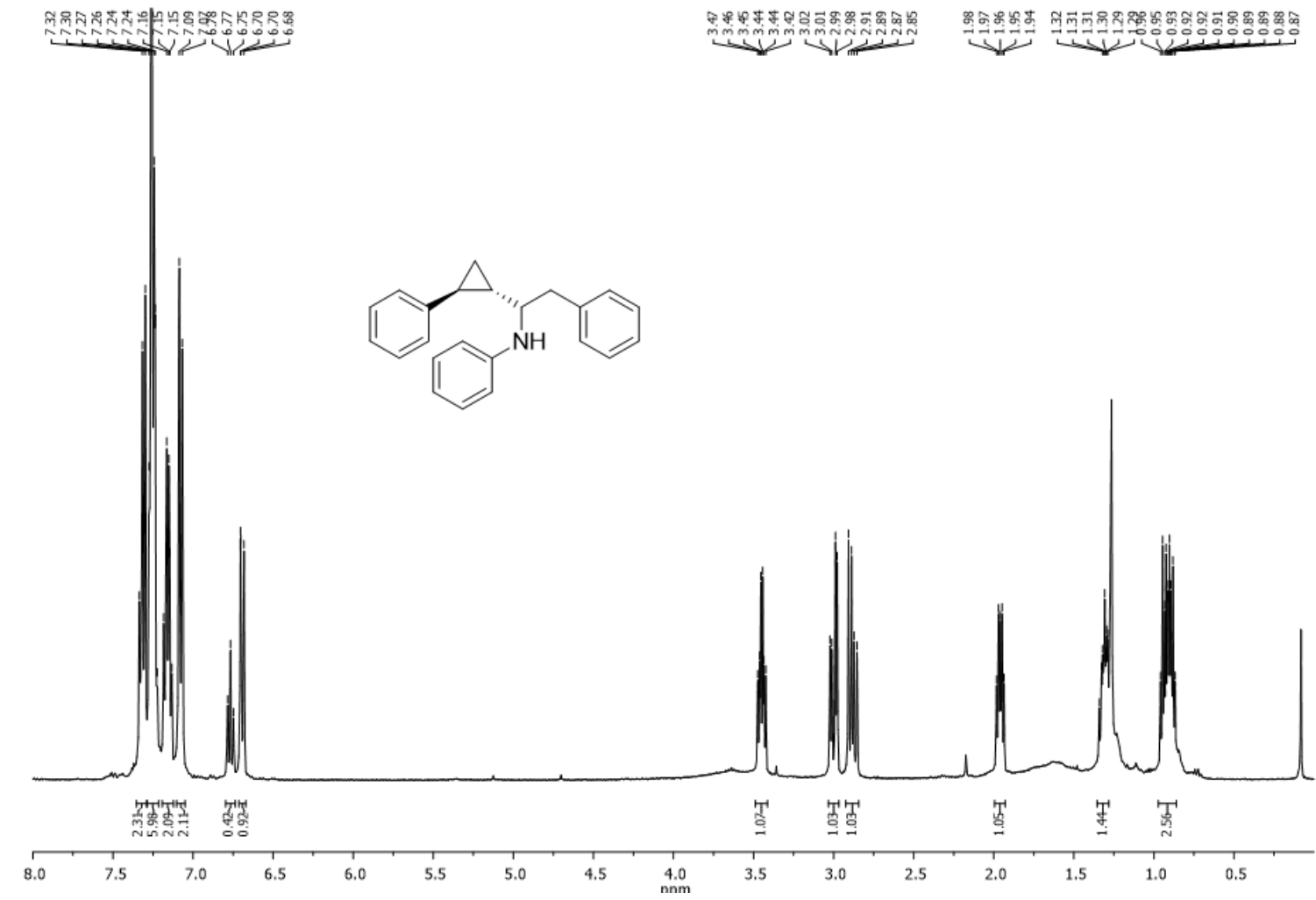

$N$-((2-phenyl-1-((1S,2S)-2-phenylcyclopropyl)ethyl)aniline (12), ${ }^{13} \mathrm{C}$ NMR (126 MHz, $\left.\mathrm{CDCl}_{3}\right)$ : ।
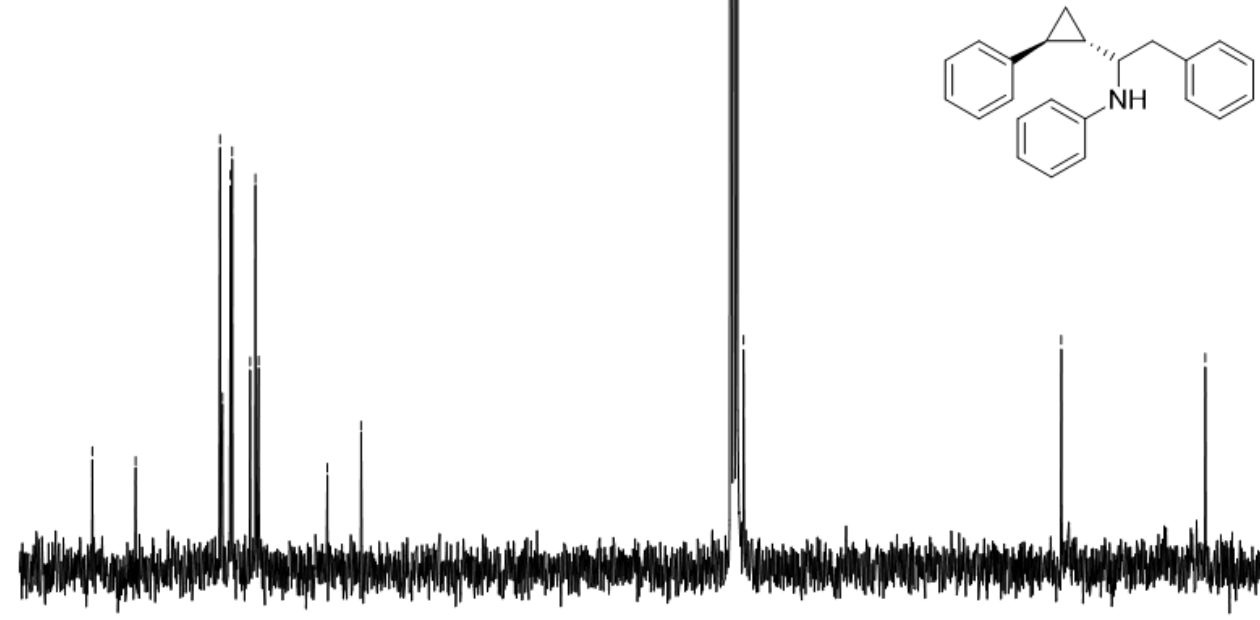

\begin{tabular}{lllllllllllllllllllllllllllllllllllllllllllllll}
\hline 50 & 145 & 140 & 135 & 130 & 125 & 120 & 115 & 110 & 105 & 100 & 95 & 90 & 85 & 80 & 75 & 70 & 65 & 60 & 55 & 50 & 45 & 40 & 35 & 30 & 25 & 20 & 15 & 10 & 5 & 0
\end{tabular} 
2-phenyl-1-((1S,2S)-2-phenylcyclopropyl)propan-1-one (13), ${ }^{1} \mathrm{H}$ NMR $\left(500 \mathrm{MHz}, \mathrm{CDCl}_{3}\right)$ :

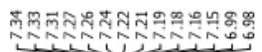

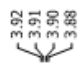

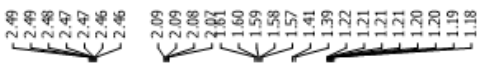

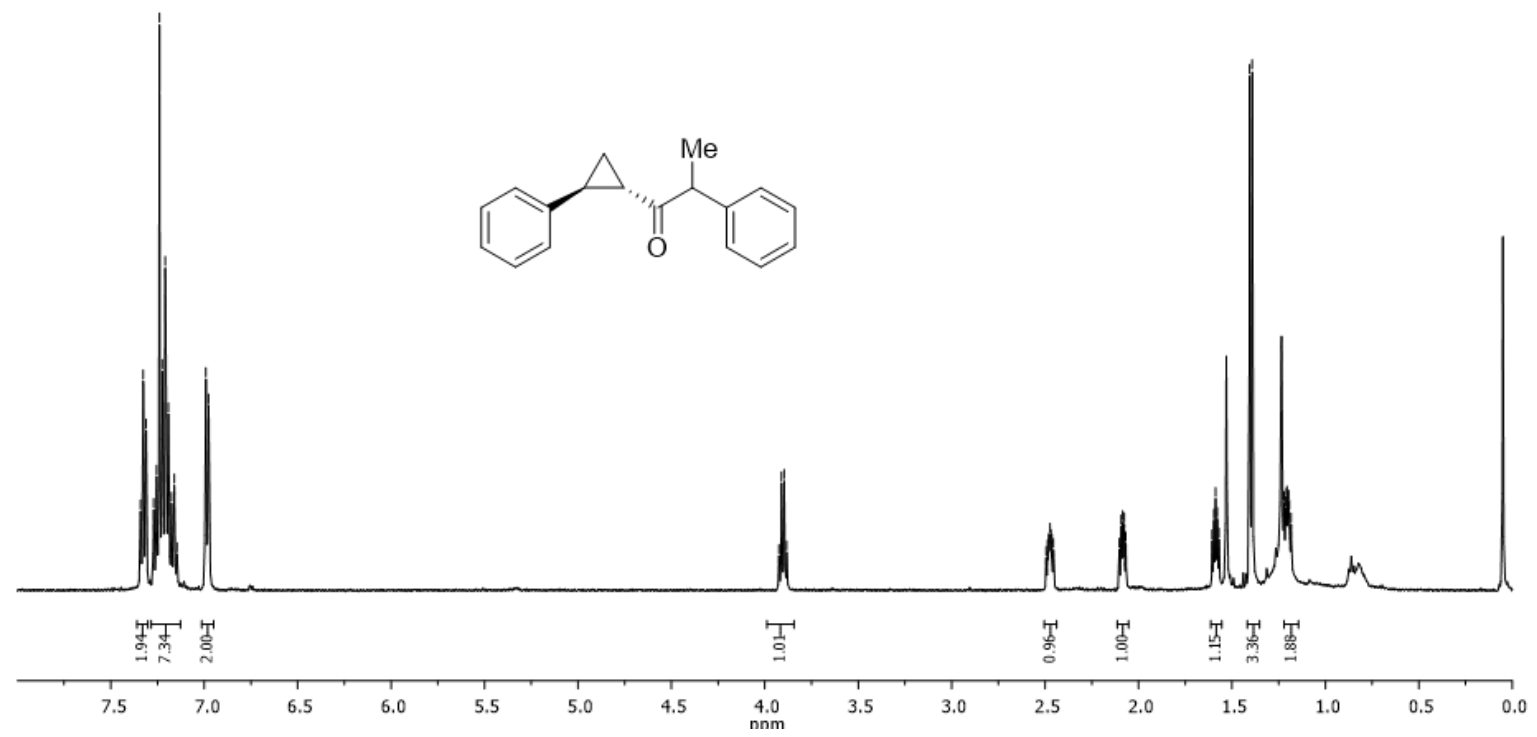

2-phenyl-1-((1S,2S)-2-phenylcyclopropyl)propan-1-one (13), ${ }^{13} \mathrm{C}$ NMR (126 MHz, $\left.\mathrm{CDCl}_{3}\right)$ :
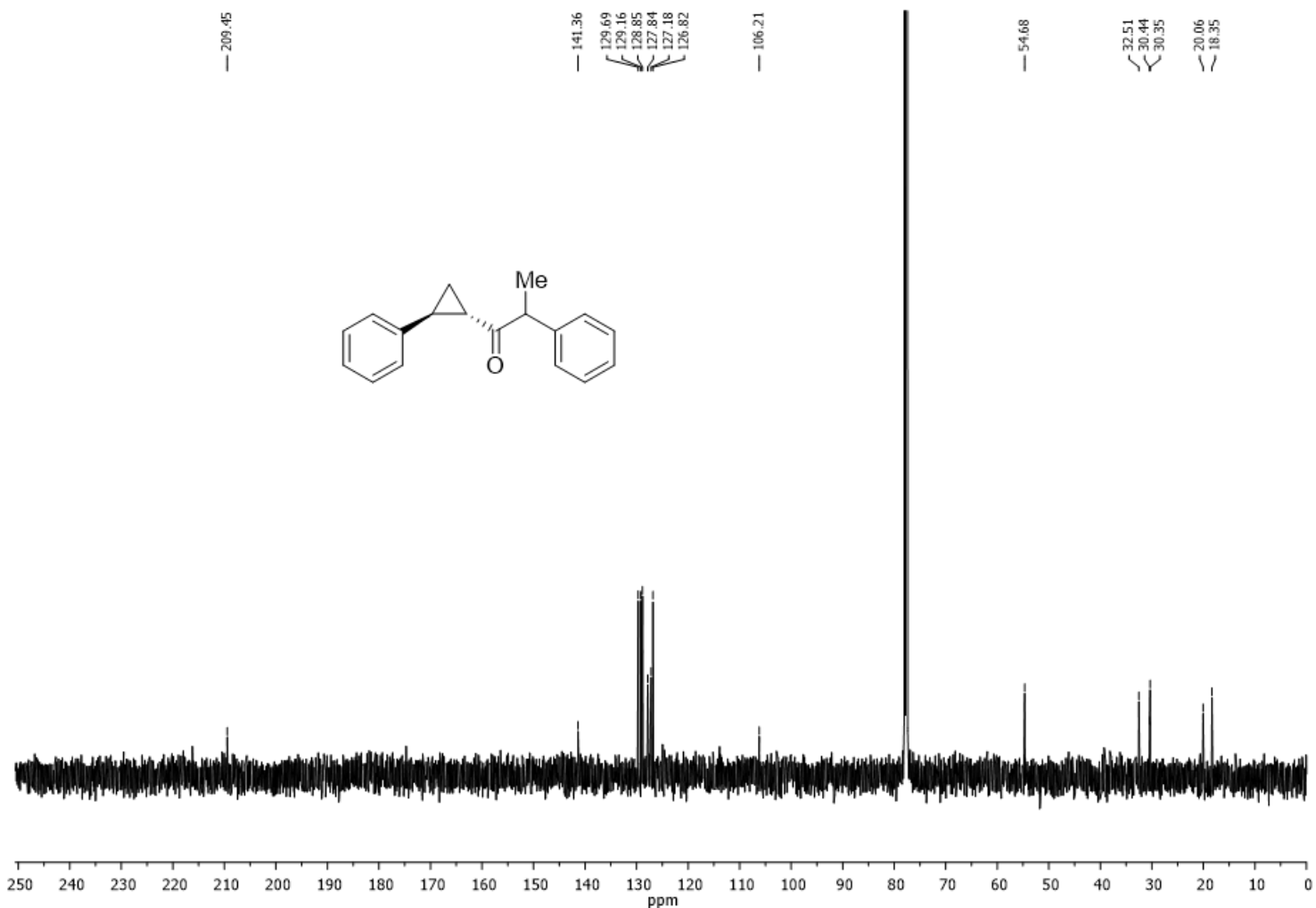
2-fluoro-2-phenyl-1-((1S,2S)-2-phenylcyclopropyl)ethan-1-one (14), ${ }^{1} \mathrm{H} \quad \mathrm{NMR} \quad(500 \mathrm{MHz}$, $\left.\mathrm{CDCl}_{3}\right)$ :

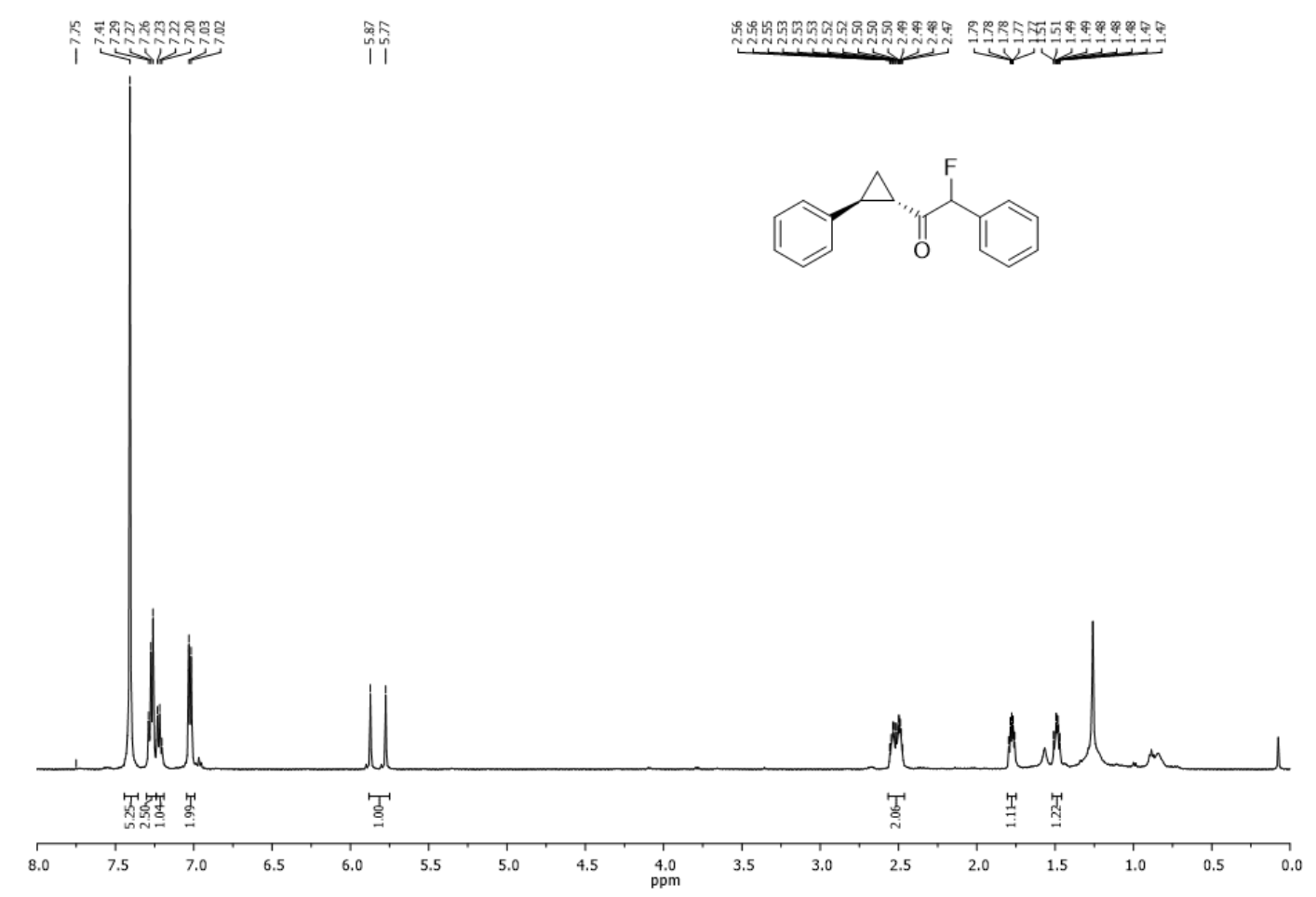

2-fluoro-2-phenyl-1-((1S,2S)-2-phenylcyclopropyl)ethan-1-one (14), ${ }^{13} \mathrm{C} \quad \mathrm{NMR} \quad(126 \mathrm{MHz}$, $\left.\mathrm{CDCl}_{3}\right)$ :

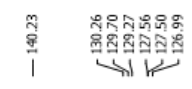

\section{g.
ga
id
i}

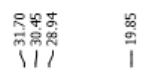
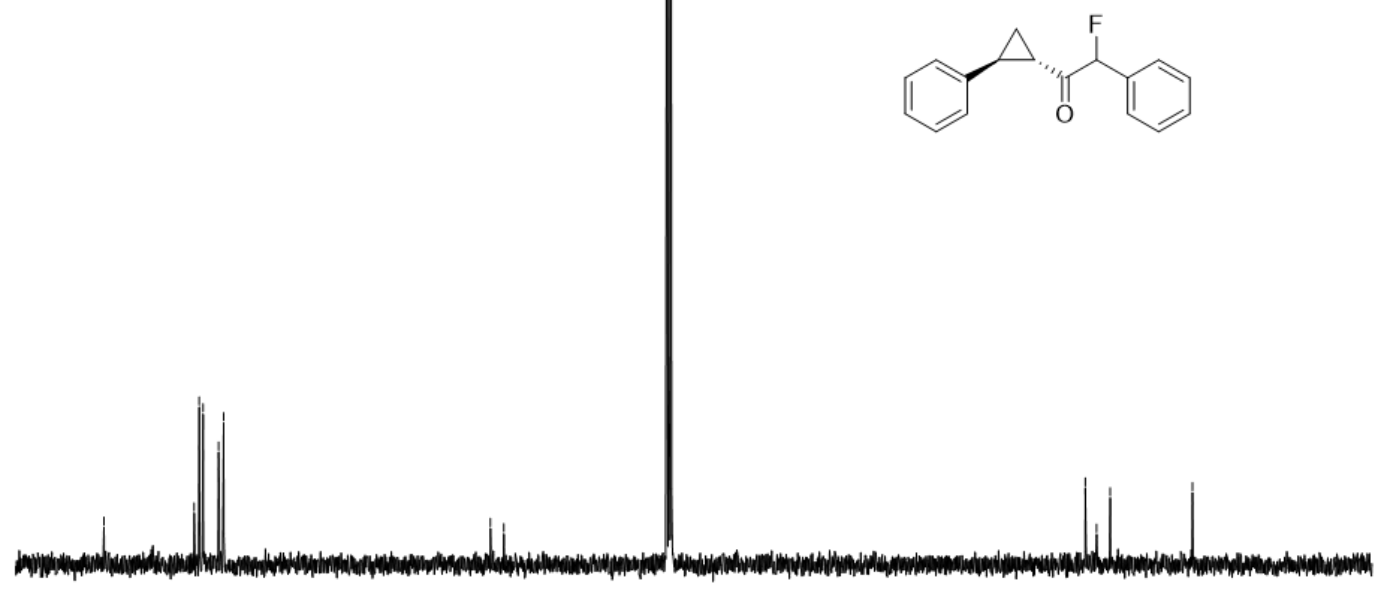

$\begin{array}{rllllllllllllllllllllllllllllllllllllllllllllll}150 & 145 & 140 & 135 & 130 & 125 & 120 & 115 & 110 & 105 & 100 & 95 & 90 & 85 & 80 & 75 & 70 & 65 & 60 & 55 & 50 & 45 & 40 & 35 & 30 & 25 & 20 & 15 & 10 & 5 & 0\end{array}$ 
2-fluoro-2-phenyl-1-((1S,2S)-2-phenylcyclopropyl)ethan-1-one (14), ${ }^{19} \mathrm{~F} \quad \mathrm{NMR} \quad(376 \mathrm{MHz}$, $\left.\mathrm{CDCl}_{3}\right)$ :

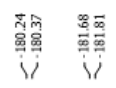
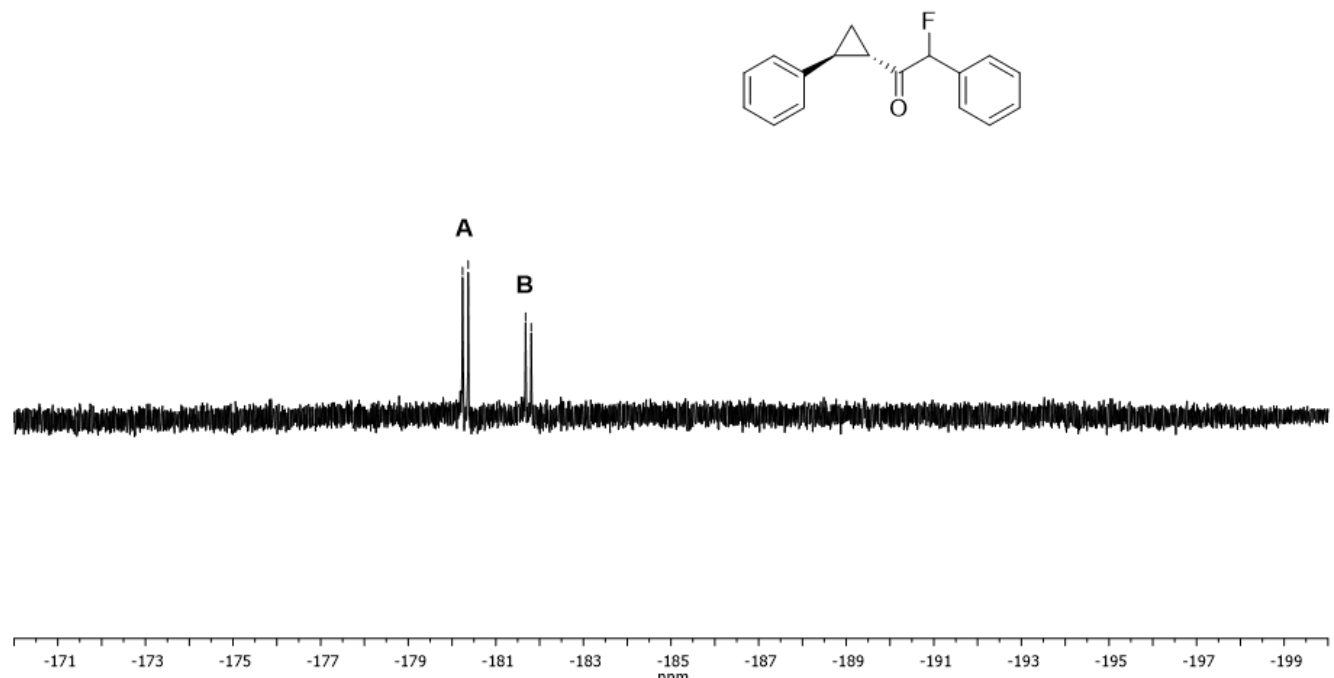
1-phenyl-2-((1S,2S)-2-phenylcyclopropyl)ethane-1,2-dione (15), ${ }^{1} \mathrm{H}$ NMR (400 MHz, $\left.\mathrm{CDCl}_{3}\right)$ :

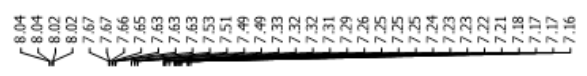

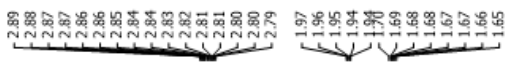

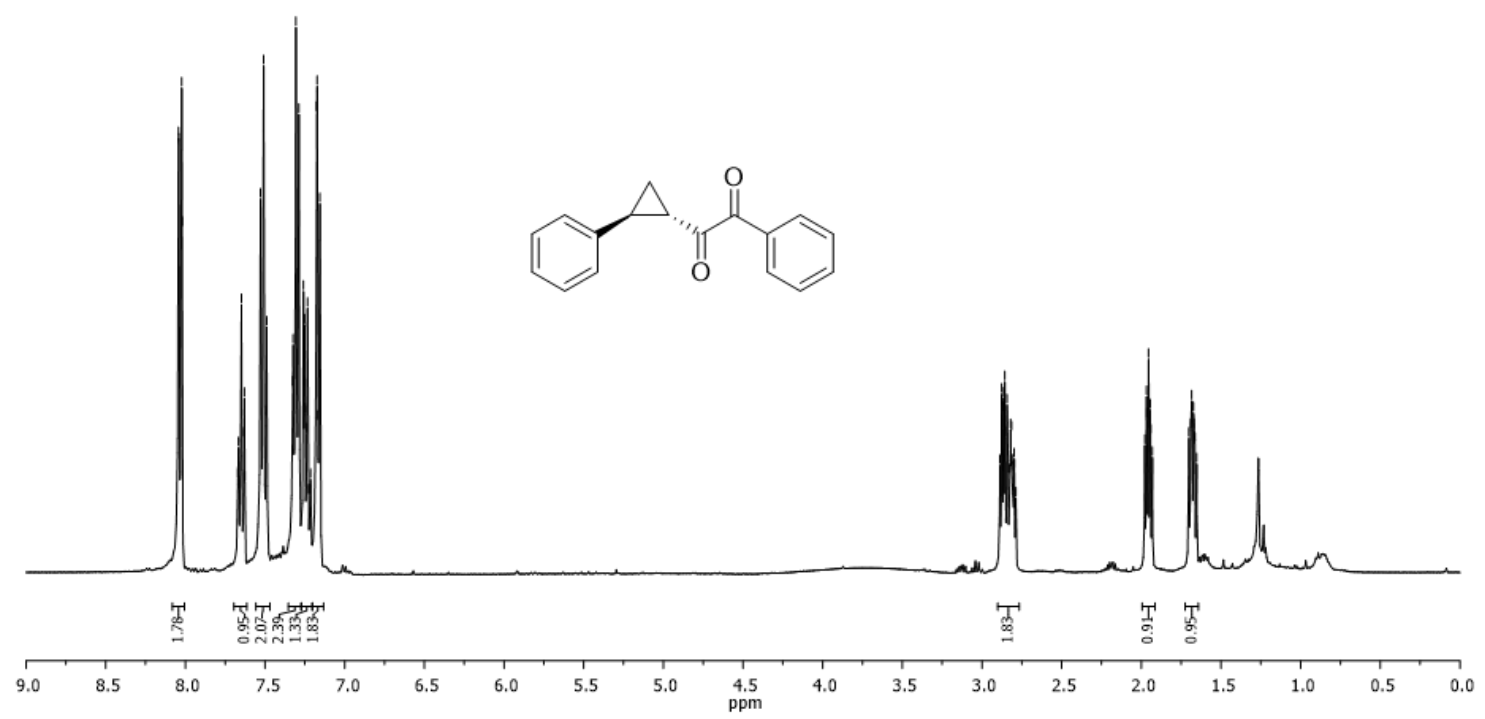

1-phenyl-2-((1S,2S)-2-phenylcyclopropyl)ethane-1,2-dione (15), $\left.{ }^{13} \mathrm{C} \mathrm{NMR} \mathrm{(101} \mathrm{MHz,} \mathrm{CDCl}_{3}\right)$ :
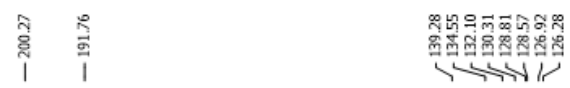

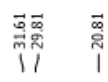
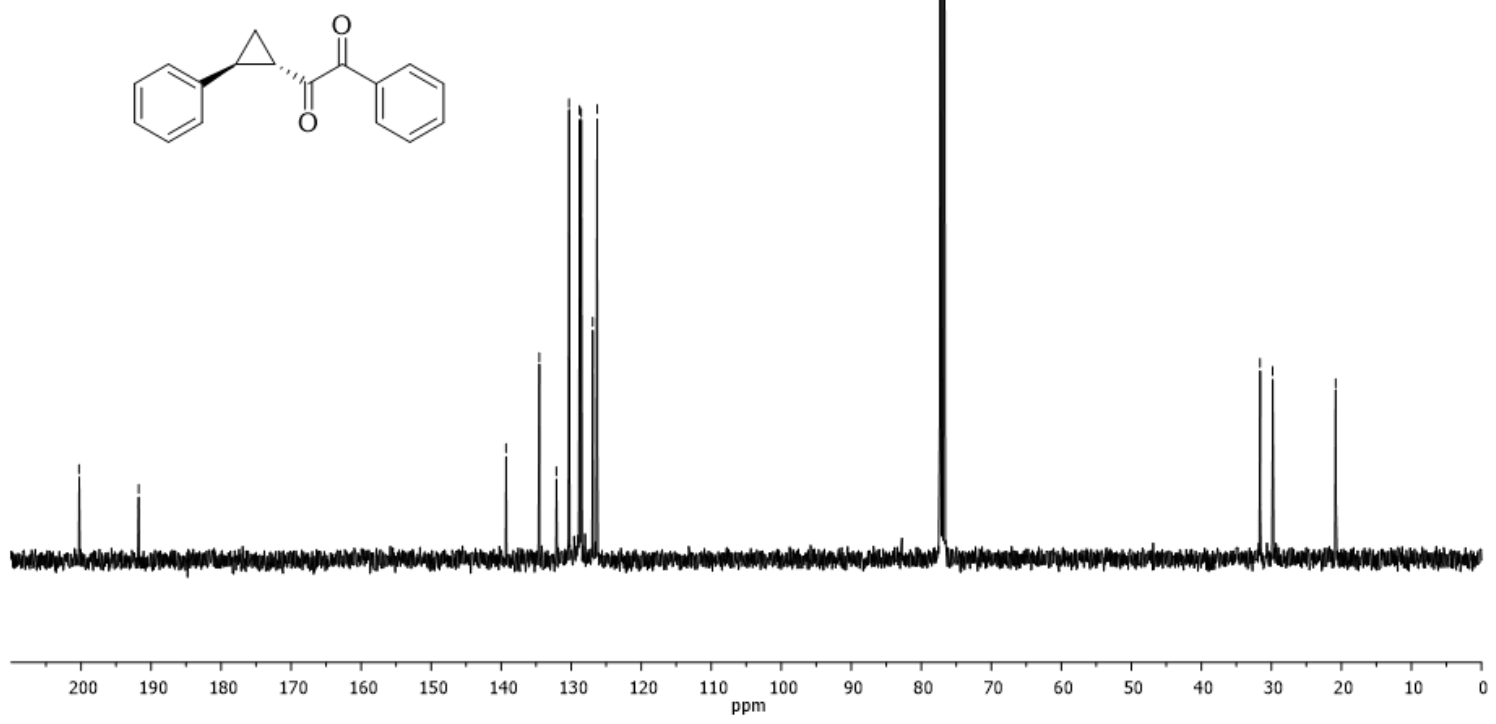

S131 
3-(dimethylamino)-2-phenyl-1-((1S,2S)-2-phenylcyclopropyl)prop-2-en-1-one (16), ${ }^{1} \mathrm{H}$ NMR $\left(500 \mathrm{MHz}, \mathrm{CDCl}_{3}\right)$ :
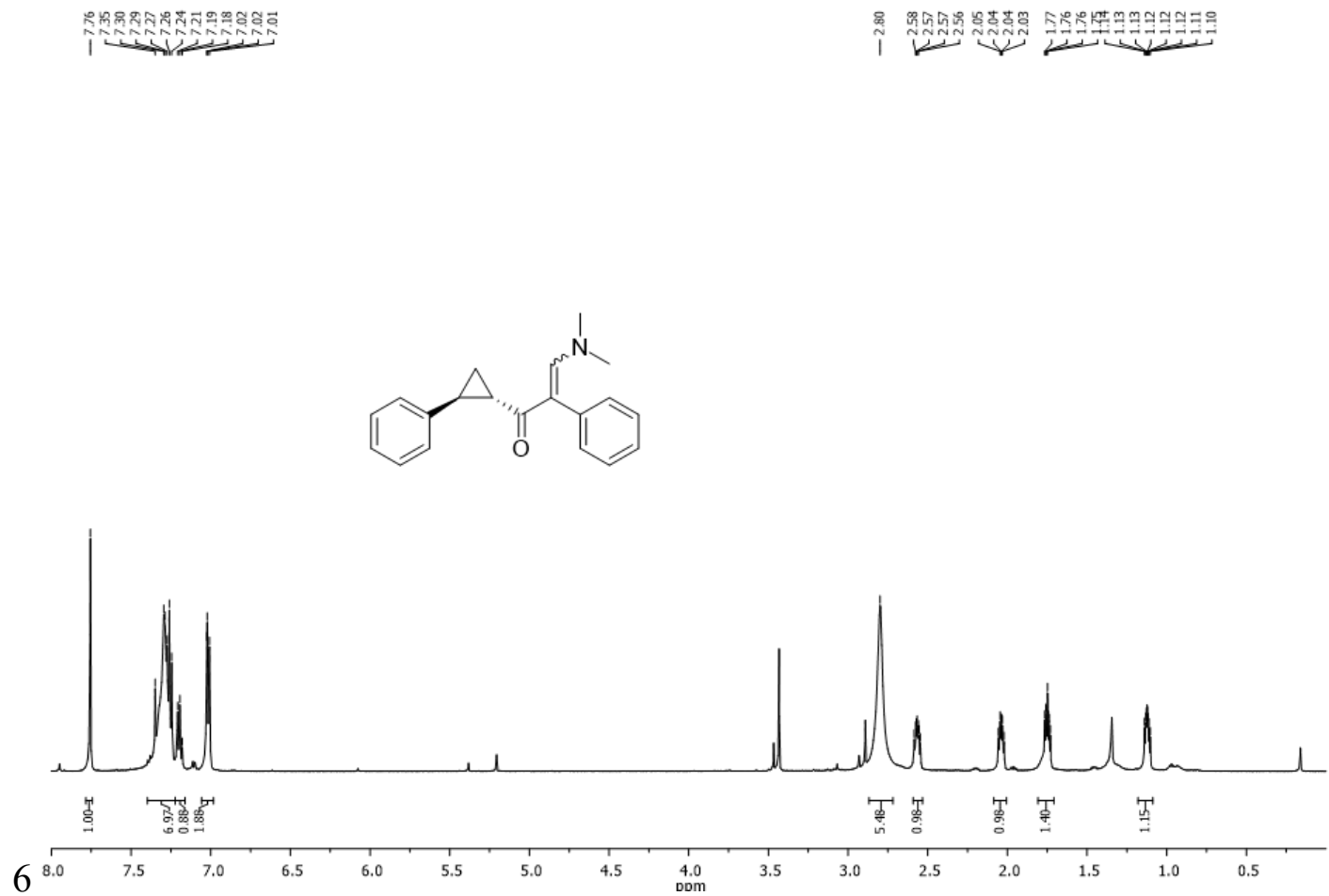

3-(dimethylamino)-2-phenyl-1-((1S,2S)-2-phenylcyclopropyl)prop-2-en-1-one $(\mathbf{1 6}),{ }^{13} \mathrm{C} \quad \mathrm{NMR}$ $\left(126 \mathrm{MHz}, \mathrm{CDCl}_{3}\right)$ :
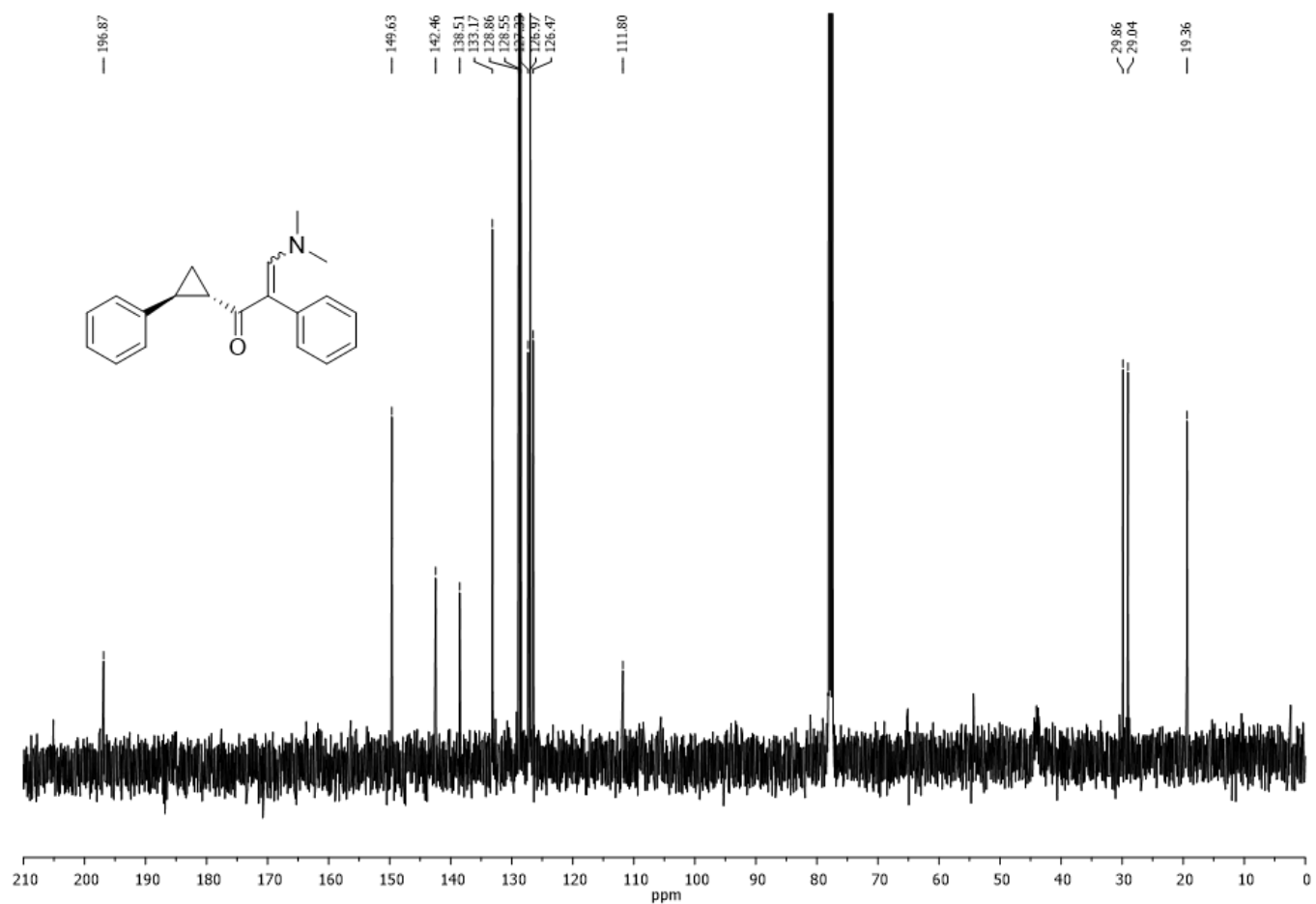
Trimethyl((2-phenyl-1-((1S,2S)-2-phenylcyclopropyl)vinyl)oxy)silane (17), ${ }^{1} \mathrm{H}$ NMR $(500 \mathrm{MHz}$, $\left.\mathrm{CDCl}_{3}\right)$ :
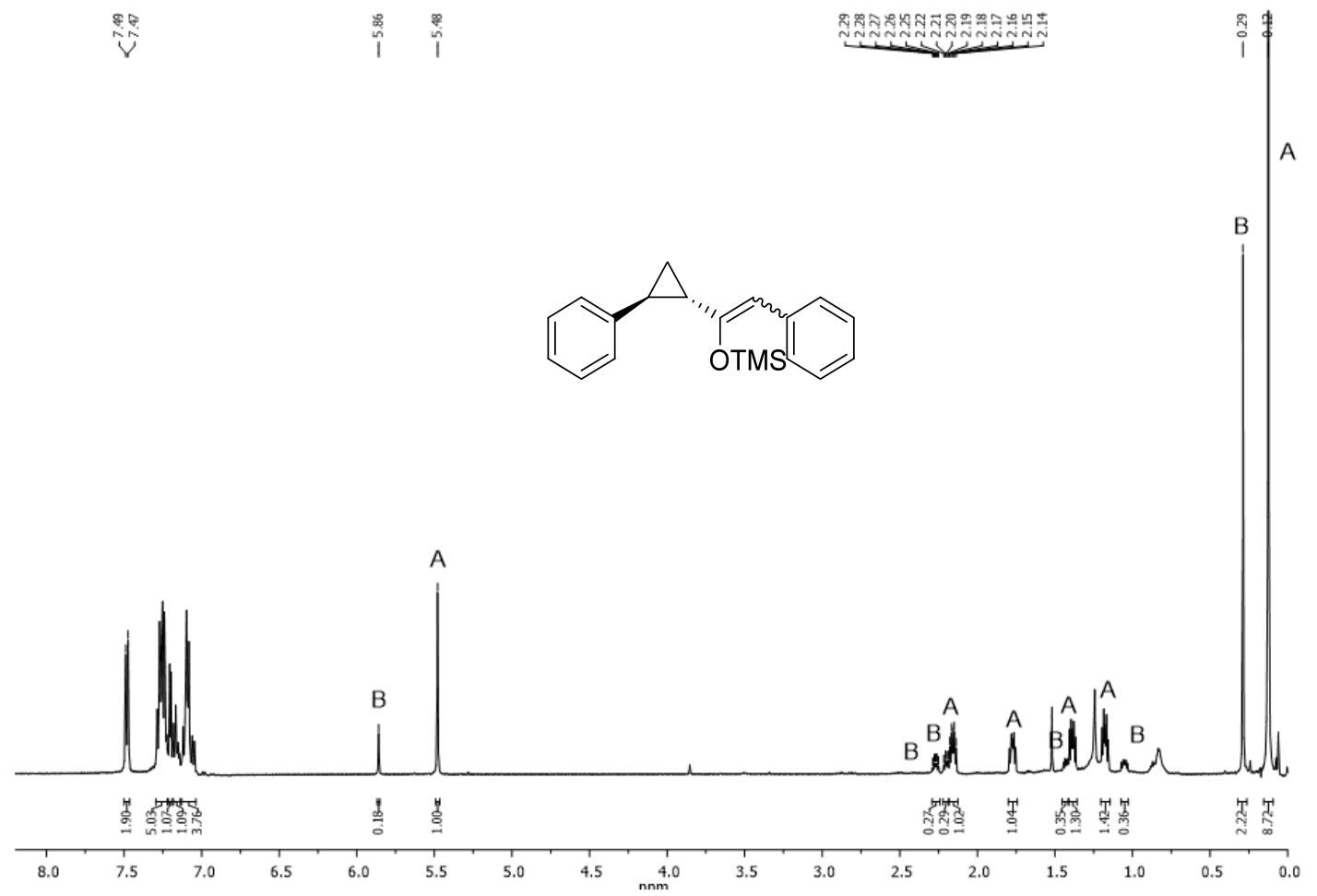

Trimethyl((2-phenyl-1-((1S,2S)-2-phenylcyclopropyl)vinyl)oxy)silane (17), ${ }^{13} \mathrm{C}$ NMR (126 MHz, $\left.\mathrm{CDCl}_{3}\right)$ :
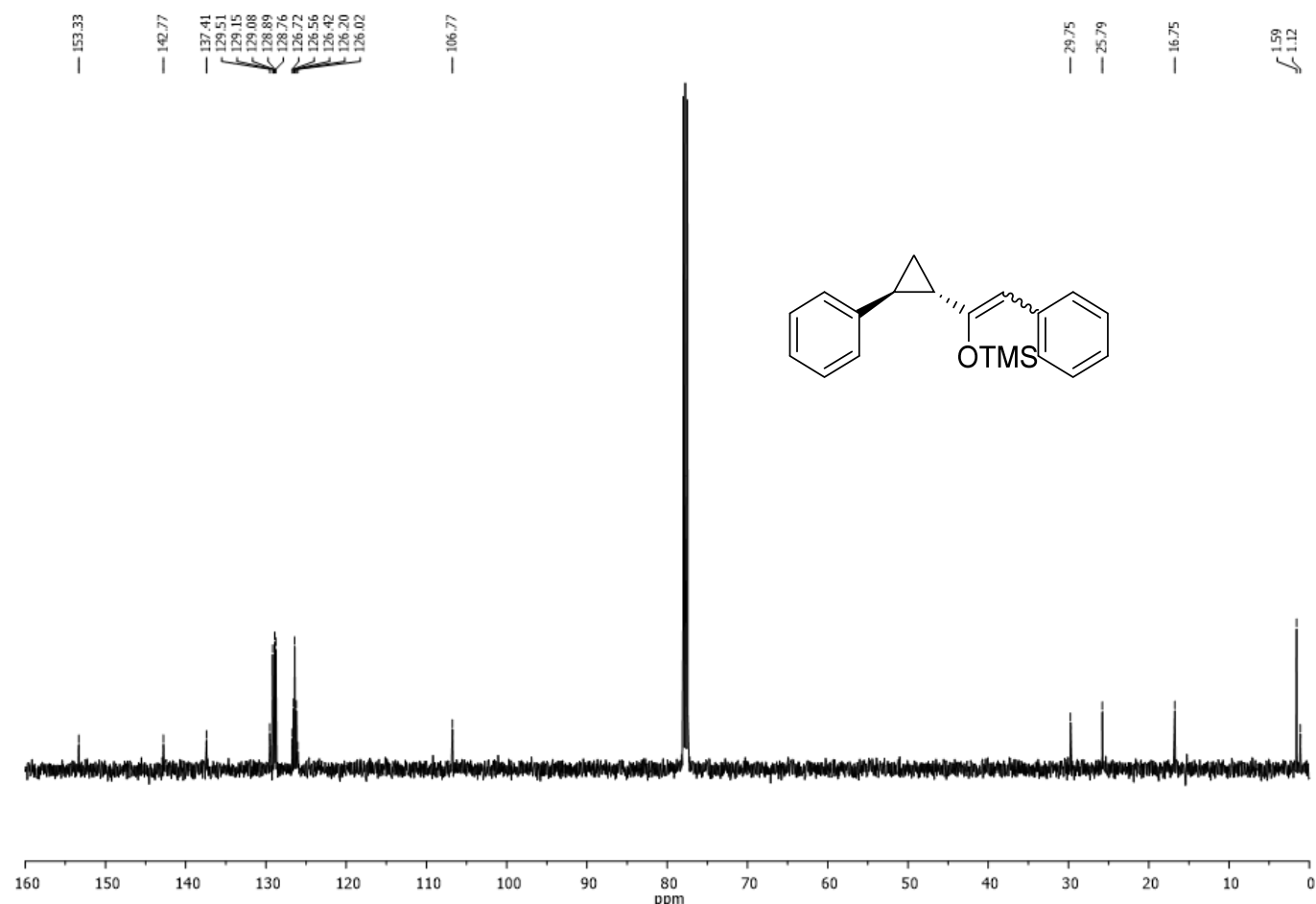
2-phenyl-3-((1S,2S)-2-phenylcyclopropyl)quinoxaline (18), ${ }^{1} \mathrm{H} \mathrm{NMR}\left(500 \mathrm{MHz}, \mathrm{CDCl}_{3}\right)$ :

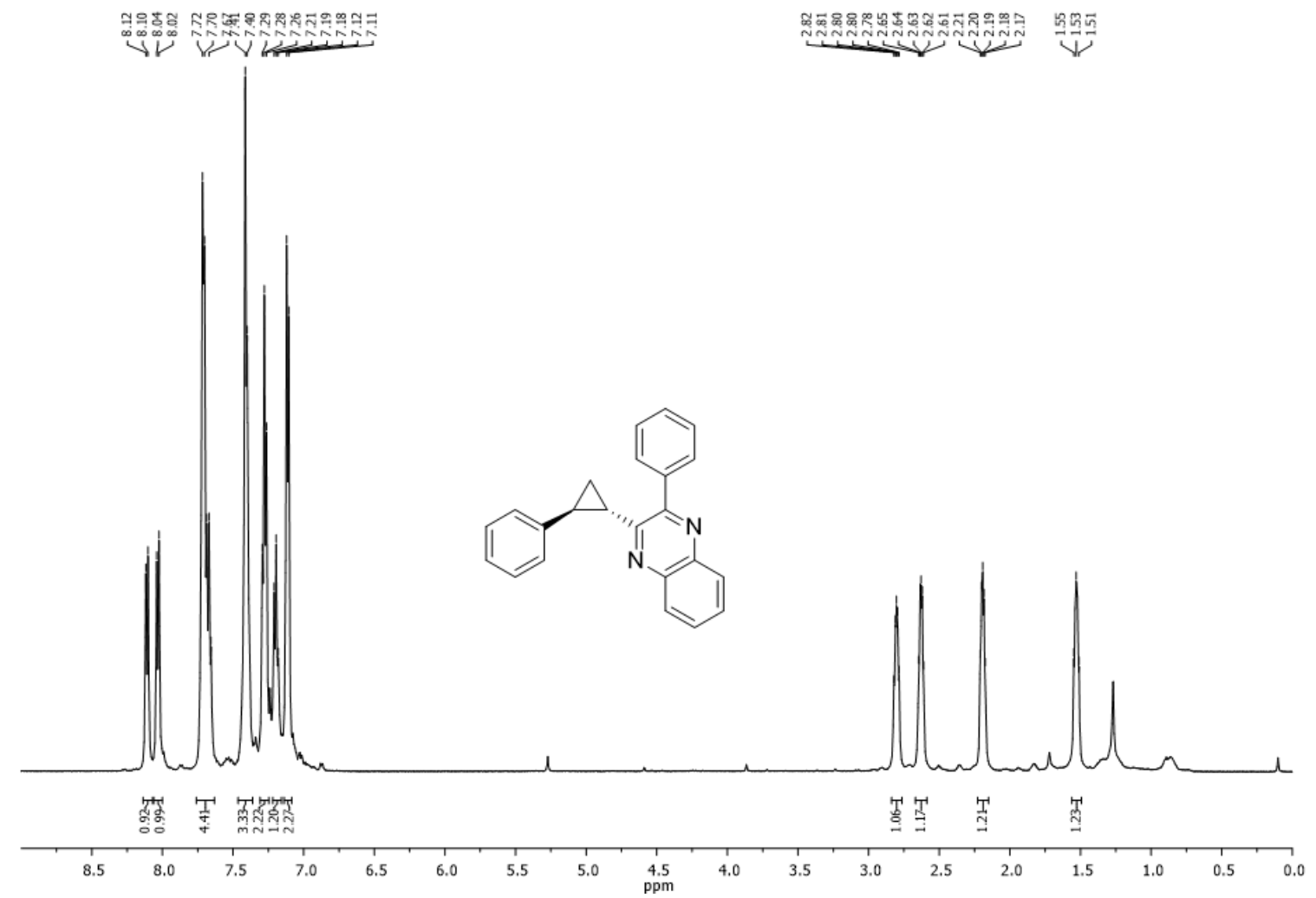

2-phenyl-3-((IS,2S)-2-phenylcyclopropyl)quinoxaline (18), ${ }^{13} \mathrm{C} \mathrm{NMR}\left(126 \mathrm{MHz}, \mathrm{CDCl}_{3}\right)$ :
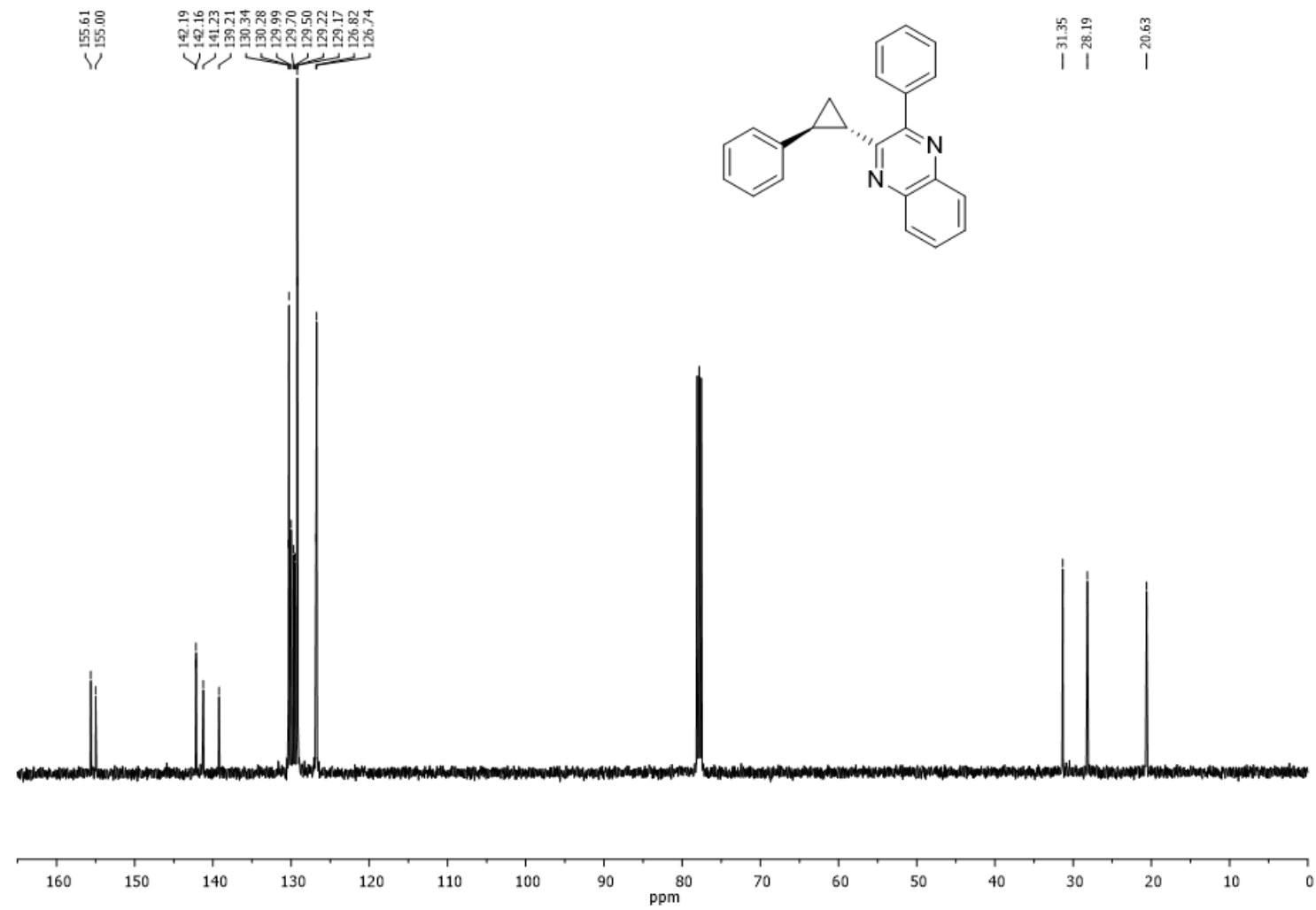


\section{References}

1. Xiao, H.-Y.; Watterson, S. H.; Langevine, C. M.; Srivastava, A. S.; Ko, S. S.; Zhang, Y.; Cherney, R. J.; Guo, W.-W.; Gilmore, J. L.; Sheppeck, J. E.; Wu, D.-R.; Li, P.; Ramasamy, D.; Arunachalam, P.; Mathur, A.; Taylor, T. L.; Shuster, D. J.; McIntyre, K. W.; Shen, D.-R.; Yarde, M.; Cvijic, M. E.; Marino, A. M.; Balimane, P. V.; Yang, Z.; Banas, D. M.; Cornelius, G.; D’Arienzo, C. J.; Warrack, B. M.; Lehman-McKeeman, L.; Salter-Cid, L. M.; Xie, J.; Barrish, J. C.; Carter, P. H.; Dyckman, A. J.; Dhar, T. G. M., Identification of Tricyclic Agonists of Sphingosine-1-phosphate Receptor 1 (S1P1) Employing Ligand-Based Drug Design. Journal of Medicinal Chemistry 2016, 59 (21), 9837-9854.

2. Imamoto, T.; Takiyama, N.; Nakamura, K.; Hatajima, T.; Kamiya, Y., Reactions of carbonyl compounds with Grignard reagents in the presence of cerium chloride. Journal of the American Chemical Society 1989, 111 (12), 4392-4398.

3. Abdel-Magid, A. F.; Carson, K. G.; Harris, B. D.; Maryanoff, C. A.; Shah, R. D., Reductive Amination of Aldehydes and Ketones with Sodium Triacetoxyborohydride. Studies on Direct and Indirect Reductive Amination Procedures1. The Journal of Organic Chemistry 1996, 61 (11), 3849-3862.

4. Stavber, G.; Zupan, M.; Stavber, S., Micellar-System-Mediated Direct Fluorination of Ketones in Water. Synlett 2009, 2009 (04), 589-594.

5. Cantley, A. M.; Welsch, M.; Ambesi-Impiombato, A.; Sanchez-Martin, M.; Kim, M.Y.; Bauer, A.; Ferrando, A.; Stockwell, B. R., Small Molecule that Reverses Dexamethasone Resistance in T-cell Acute Lymphoblastic Leukemia (T-ALL). ACS Medicinal Chemistry Letters 2014, 5 (7), 754-759.

6. Ghosh, P.; Mandal, A., Greener approach toward one pot route to pyrazine synthesis. Green Chemistry Letters and Reviews 2012, 5 (2), 127-134.

7. Sheldrick, G., SHELXT - Integrated space-group and crystal-structure determination. Acta Crystallographica Section A 2015, 71 (1), 3-8.

8. Sheldrick, G., Crystal structure refinement with SHELXL. Acta Crystallographica Section C 2015, 71 (1), 3-8. 